UC-814

Issued: December 1995

Distribution and Chemistry of

Fracture-Lining Minerals at

Yucca Mountain, Nevada

Barbara A. Carlos

Steve J. Chipera

David L. Bish 


\section{DISCLAMMER}

Portions of this document may be illegible in electronic image products. Images are produced from the best available original document. 


\title{
DISTRIBUTION AND CHEMISTRY OF FRACTURE-LINING MINERALS AT YUCCA MOUNTAIN, NEVADA
}

\author{
by \\ Barbara A. Carlos, Steve J. Chipera, and David L. Bish
}

\begin{abstract}
Yucca Mountain, a >1.5-km-thick sequence of tuffs and subordinate lavas in southwest Nevada, is being investigated as a potential high-level nuclear waste repository site. Fracturelining minerals have been studied because they may provide information on past fluid transport and because they may act as natural barriers to radionuclide migration within the fractures. Cores from seven drill holes have been studied to determine the distribution and chemistry of minerals lining fractures at Yucca Mountain.

Fracture-lining minerals in tuffs of the Paintbrush Group, which is above the static water level at Yucca Mountain, are highly variable in distribution, both vertically and laterally across the mountain, with the zeolites mordenite, heulandite, and stellerite widespread in fractures even though the tuff matrix is generally devitrified and nonzeolitic. Where heulandite occurs as both tabular and prismatic crystals in the same fracture, the two morphologies have different compositions, suggesting multiple episodes of zeolite formation within the fractures. Manganese-oxide minerals within the Paintbrush Group are rancieite and lithiophorite. The silica polymorphs (quartz, tridymite, and cristobalite) generally exist in fractures where they exist in the matrix, suggesting that they formed in the fractures at the same time they formed in the matrix. Fluorite, calcite, and opal occur over tridymite in some lithophysal cavities. Calcite also occurs over zeolites in fractures unrelated to lithophysal cavities and is often the youngest mineral in a given fracture. The clays smectite, palygorskite, and sepiolite are common in fractures in the Paintbrush Group in drill core USW GU-3; smectite is an abundant fracturecoating mineral in all drill cores at Yucca Mountain.

In fractures in the Calico Hills Formation and the Crater Flat Group, zeolites generally exist mainly where the matrix is also zeolitic, although mordenite does occur as fracture linings in some devitrified intervals of the Crater Flat Group as well. Clinoptilolite and mordenite occur in fractures in tuffs containing clinoptilolite; analcime is limited to fractures in tuff intervals containing analcime. These data suggest that the formation of fracture-lining zeolites in the Calico Hills Formation and the Crater Flat Group may have been coincident with the original alteration of the tuffs. Manganese-oxide minerals in the Calico Hills Formation and the Crater Flat Group occur principally in devitrified tuff intervals and are mainly cryptomelane/hollandite family minerals, although lithiophorite, todorokite, pyrolusite, rancieite, and aurorite also occur.

The calcic compositions of the zeolites and manganese-oxide minerals in the Paintbrush Group and the sodic and potassic compositions of the zeolites and potassic manganese-oxide minerals in the Calico Hills Formation and Crater Flat Group suggest that fluid compositions in the Paintbrush Group differed from those in deeper tuffs. Although matrix and fracture-lining zeolites may have formed under similar conditions (saturation and/or lateral flow) in the Calico Hills Formation and below, the fractures in the Paintbrush Group contain zeolites where there are none in the matrix, suggesting that for these minerals, localized mineral deposition resulted from fracture flow within the unsaturated zone.
\end{abstract}




\section{INTRODUCTION}

Yucca Mountain in southwest Nevada (Figure 1) is being investigated as a potential site for a high-level nuclear waste repository partly because the thick sequences of zeolitic tuffs provide a natural barrier to the migration of some radionuclides, notably the alkali and alkalineearth elements (Thomas, 1987). The potential repository horizon is in the lower portion of the Topopah Spring Tuff of the Paintbrush Group, $>300 \mathrm{~m}$ below the surface of the mountain and $150 \mathrm{~m}$ above the static water level (SWL). Because fractures represent both past and potential future transport pathways, fracture-lining minerals are being studied to provide information on transport within Yucca Mountain and on their use as possible barriers to radionuclide migration. This report includes descriptions of fracture coatings in core from seven holes drilled before 1984 (USW G-1, G-2, GU-3, G-3, G-4, UE-25a\#1, and UE-25b\#1). Of these, G-3 is essentially a continuation of GU-3, and UE-25b\#1 is a continuation of UE 25 a\#1. Both holes were stepped over slightly from the original hole and cored deeper below the SWL. This study of fracture-lining minerals was limited to the Paintbrush Group, the Calico Hills Formation, and the Crater Flat Group, the units most likely to be encountered by future fluid flow between the potential repository and the accessible environment. Although these cores were drilled before development of an approved quality assurance (QA) program, they are useful in developing conceptual models of mineral distribution that may be validated, modified, or discarded as data are collected from new cores. Previous publications on Yucca Mountain fracture coatings have focused on single drill cores or single mineral suites (see Carlos, 1985; Carlos, 1987; Carlos, 1989; Carlos et al., 1991; Carlos et al., 1993; Carlos et al., 1995). This report represents a summary of existing fracture mineralogy data at Yucca Mountain.

\section{BACKGROUND GEOLOGY}

Yucca Mountain is composed of a $>1.5-\mathrm{km}$-thick sequence of tuffs and subordinate lavas (Scott et al., 1983; Carr et al., 1986). The tuff units include partially to densely welded devitrified tuff, densely welded vitrophyre, and nonwelded vitric tuff which in places has been extensively altered to zeolite minerals. Detailed descriptions of these ash flow tuffs are given in Lipman et al. (1966), Byers et al. (1976), and Carr et al. (1986), and regional geology of the volcanic field and ages of the tuffs are discussed in Sawyer et al. (1994). The stratigraphy of the drill cores examined in this study has been described by Spengler et al. (1979), Spengler et al. (1981), Lobmeyer et al. (1983), Maldonado and Koether (1983), Scott and Castellanos 


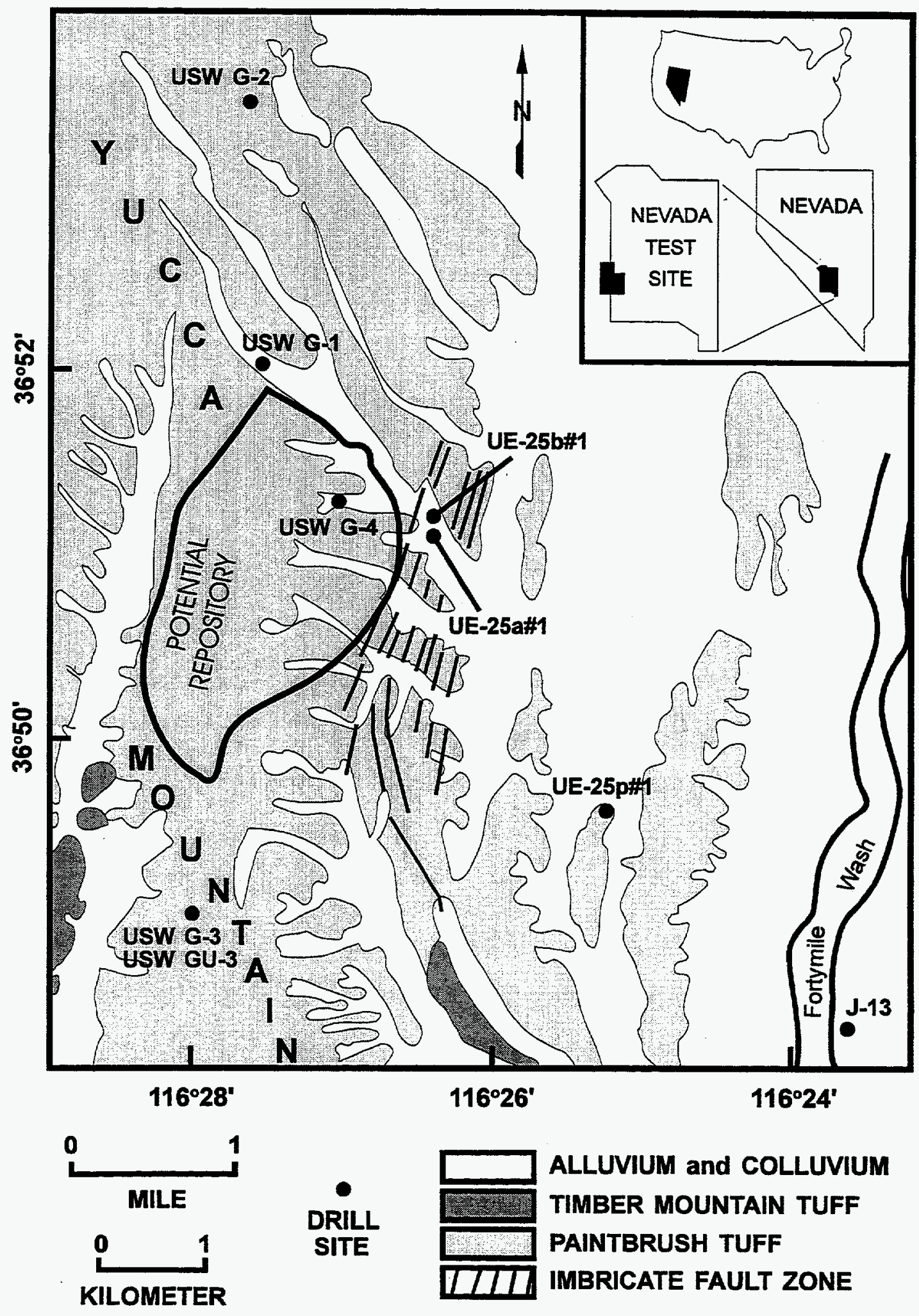

Figure 1. Location map showing Yucca Mountain and the drill holes mentioned in this report. 
(1984), and Spengler and Chornack (1984). From top to bottom, the units at Yucca Mountain are the Tiva Canyon, Yucca Mountain, Pah Canyon, and Topopah Spring Tuffs of the Paintbrush Group; the Calico Hills Formation; the Prow Pass, Bullfrog, and Tram Tuffs of the Crater Flat Group; and older tuffs and lavas that are not included in this study (Figure 2). The SWL falls within the Crater Flat Group beneath most of Yucca Mountain.

The mineralogy of the tuff units is described in Bish and Chipera (1989). Alteration of Yucca Mountain tuffs is discussed by Broxton et al. (1987), who describe four depth-related zones of diagenetic alteration. The zones can be identified by key minerals in the rock matrix in the partly welded to nonwelded intervals. Zone I is above the SWL and is characterized by the presence of glass and isolated occurrences of heulandite. Zone II begins somewhat above the present SWL and is characterized by the presence of clinoptilolite and mordenite, which replace glass. Zone III is characterized by the presence of analcime, although clinoptilolite and mordenite persist at least in the upper part of this zone, along with quartz and authigenic potassium feldspar. Zone IV contains albite, which replaces analcime; quartz and potassium feldspar are also present. Broxton et al. (1987) noted that alteration is more intense toward the north and attributed the alteration to a thermal pulse related to the Timber Mountain-Oasis Valley caldera complex located north of Yucca Mountain. Bish (1989) used illite/smectite interstratifications to infer the temperatures to which the different zones had been subjected. The distribution of illite/smectite and K/Ar age dates of illite of about $11 \mathrm{Ma}$ support the hypothesis that alteration was the result of a regional increase in geothermal gradient related to the Timber Mountain volcanism. No evidence of additional hydrothermal alteration since that time has been found (Bish and Aronson, 1993).

\section{ANALYTICAL METHODS}

Cores from seven drill holes (USW G-1, G-2, GU-3, G-3, G-4, UE-25a\#1, and UE25b\#1) representing five different localities on and near Yucca Mountain (Figure 1) were examined to determine the distribution of fracture-lining minerals. More than 500 samples from the Paintbrush Group, the Calico Hills Formation, and the Crater Flat Group were examined in this study. Fracture coatings were first examined using a binocular microscope to choose samples for grain mounts, thin sections, and scanning electron microscope (SEM) studies. Samples included closed fractures for which thin sections could be made, crystals with identifiable morphology that could be chipped off the fracture surface for grain mounts, and fragments of open fractures that were ground to a flat surface and polished. Powders for X-ray 


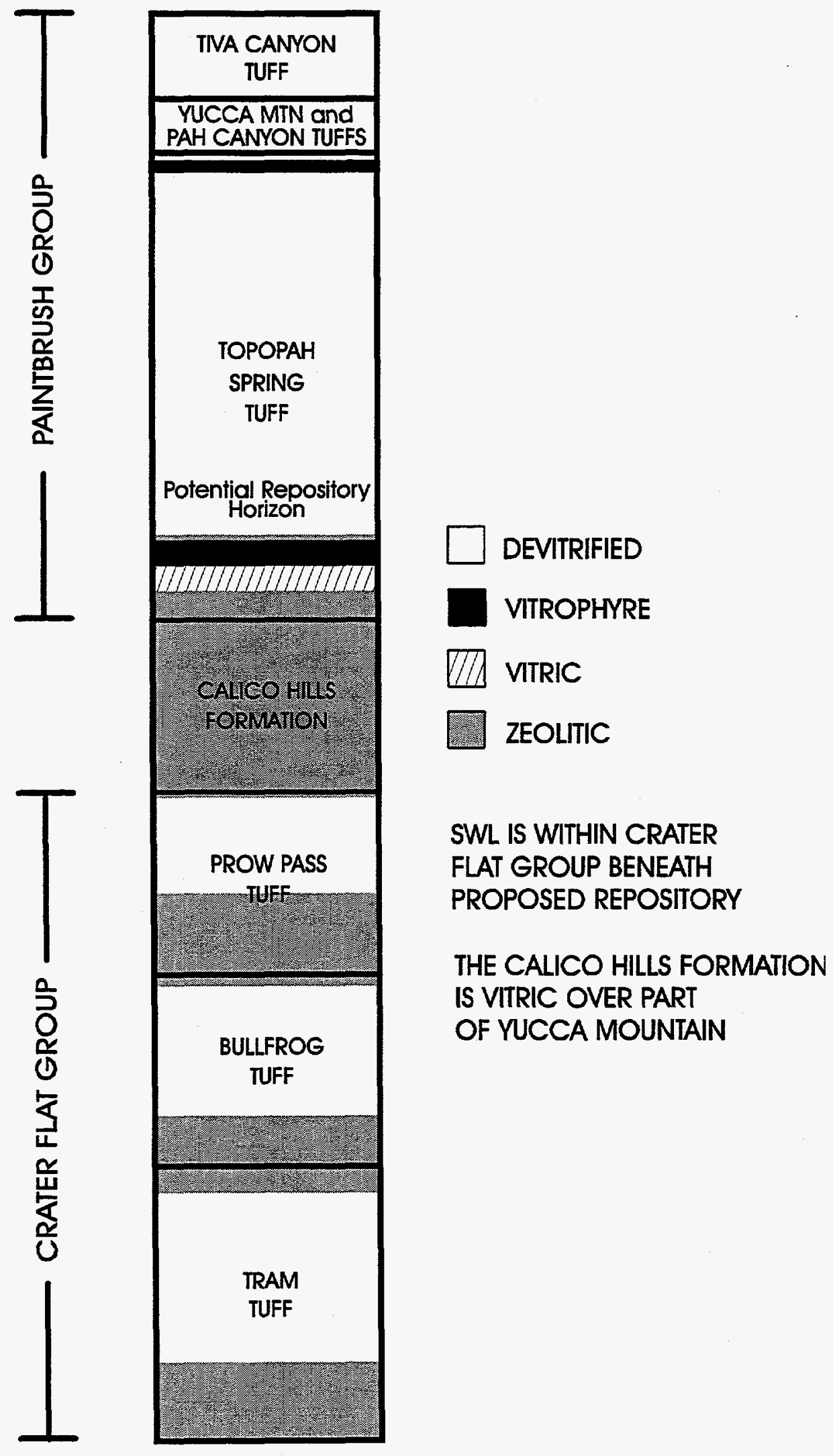

Figure 2. Generalized stratigraphy of the tuff units studied at Yucca Mountain. 
powder diffraction (XRD) analysis were scraped from surfaces and then hand picked under a binocular microscope to remove rock-matrix contamination. XRD analyses were obtained with Siemens D-500 powder diffractometers using $\mathrm{Cu} \mathrm{K \alpha}$ radiation and incident- and diffractedbeam Soller slits. Samples were mounted either as powders pressed into a cavity machined into an aluminum or glass sample plate or by suspension in deionized water or acetone and sedimentation onto an off-axis cut (zero-background) quartz plate. Samples were typically run from $2^{\circ}$ to $70^{\circ} 2 \theta$ using $0.02^{\circ}$ steps with typical count times of 16 s/step.

Open fracture surfaces were studied with an ISI model DS-130 SEM equipped with a Tracor Northern Series II energy-dispersive X-ray (EDX) analytical system. Secondary-electron images (SEI) and backscattered-electron images (BEI) were acquired at magnifications as high as $10,000 \times$ using accelerating voltages between 19 and $29 \mathrm{kV}$. Thin sections across filled fractures, polished chips, and grain mounts of crystals scraped from fracture surfaces were examined using a Tracor Northern SEM, model ADEM, equipped with an integrated EDX analytical system. SEI and BEI were collected at magnifications as high as $20,000 \times$ using accelerating voltages of 15 or $20 \mathrm{kV}$. EDX analyses assisted in the identification of minerals.

Samples for chemical analysis were selected to represent most of the different fracturelining minerals from the different intervals. Quantitative elemental analyses of thin sections, polished chips, and grain mounts were acquired on Cameca MBX and Cameca SX-50 electronprobe microanalyzers using wavelength-dispersive spectrometry. Both instruments were operated at an accelerating voltage of $15 \mathrm{kV}$ and a beam current of $15 \mathrm{nA}$. A 10- to 20- $\mu \mathrm{m}$ rastered beam, combined with movement of the sample under the raster, was used to minimize Na migration during analysis. Compositions were calculated using Bence-Albee correction methods (Bence and Albee, 1968) for the Cameca MBX instrument and using PAP correction methods (Pouchou and Pichoir, 1985) for the Cameca SX-50 instrument.

\section{RESULTS}

The most common fracture-lining minerals at Yucca Mountain are silica polymorphs, zeolites, and $\mathrm{Mn}$ oxides, with lesser amounts of clay minerals, calcite, fluorite, and Fe oxides. In general, the fracture-lining zeolites at Yucca Mountain correlate with the diagenetic zone in which the fractures occur. Appendix I presents the results of XRD analyses of fracture-lining minerals in these drill cores. This table is not all-inclusive for the mineral distributions in fractures at Yucca Mountain because many of the results listed in this report are from optical and SEM analyses of fracture samples. For example, calcite is readily identified by its 
morphology and does not require XRD analyses for positive identification. Figures $3 a$ through 3e graphically represent the mineral distribution with depth for the more significant minerals found in fractures at Yucca Mountain. These figures represent a compilation of XRD results (locations for the samples analyzed by XRD are shown on the right-hand axis of the figures) and SEM and optical determinations. The zeolite minerals clinoptilolite and heulandite are isostructural and are not readily distinguishable. No distinction between the two species was made in Appendix I or Figures 3a through 3e. However, chemical analyses of the clinoptilolite/heulandite-group minerals present in the Paintbrush Group show calcium-dominant cation compositions and Si:Al ratios of 3.4 to 3.8 indicative of heulandite (Boles, 1972). Heulandite samples from fractures in USW G-4 were heated to $450^{\circ} \mathrm{C}$ for 15 hours (Carlos, $1985)$ to determine if their structure would collapse as expected for heulandite (Mumpton, 1960). Their response to heating (partial collapse) and their chemical compositions categorize these fracture coatings as Group-2 heulandites (Alietti, 1972; Boles, 1972). This response compares well with the results of Chipera et al. (1995b) who found Group-2 heulandites in a 3-m-thick zeolitized zone in drill core UE-25 UZ\#16 (above the upper vitrophyre of the Topopah Spring Tuff). Chemical analyses of clinoptilolite-group minerals below the Paintbrush Group indicate that they are clinoptilolite and not heulandite.

The silica polymorphs quartz, cristobalite, and tridymite are common in fractures in devitrified tuff intervals, and their distribution corresponds to the occurrence of these polymorphs in the host matrix. Opal is common in fractures in the Tiva Canyon Tuff and in vitrophyres in the Paintbrush Group and is less abundant in other intervals. Opal-CT occurs with mordenite and clinoptilolite in the Calico Hills Formation and Crater Flat Group. Manganese-oxide minerals occur in fractures in the moderately to densely welded vitric and devitrified tuffs throughout the volcanic sequence at Yucca Mountain and are less common in zeolitic zones. The most common iron-oxide mineral in fractures is hematite. It occurs as redbrown staining and intergrown with silica and manganese oxides in welded, devitrified intervals. Specular hematite occurs in and near lithophysal cavities. Calcite and fluorite occur over tridymite in lithophysal cavities in several drill cores, most frequently in UE-25a\#1 and USW GU-3, but the abundance of these minerals varies laterally across Yucca Mountain. Calcite commonly occurs in fractures as well as in lithophysal cavities; however, fluorite shows limited occurrences in fractures and is most common in USW GU-3. Both are most abundant in, but are not limited to, the Paintbrush Group. Smectite is fairly ubiquitous in fractures throughout the volcanic sequence at Yucca Mountain. The clay minerals palygorskite, sepiolite, and 


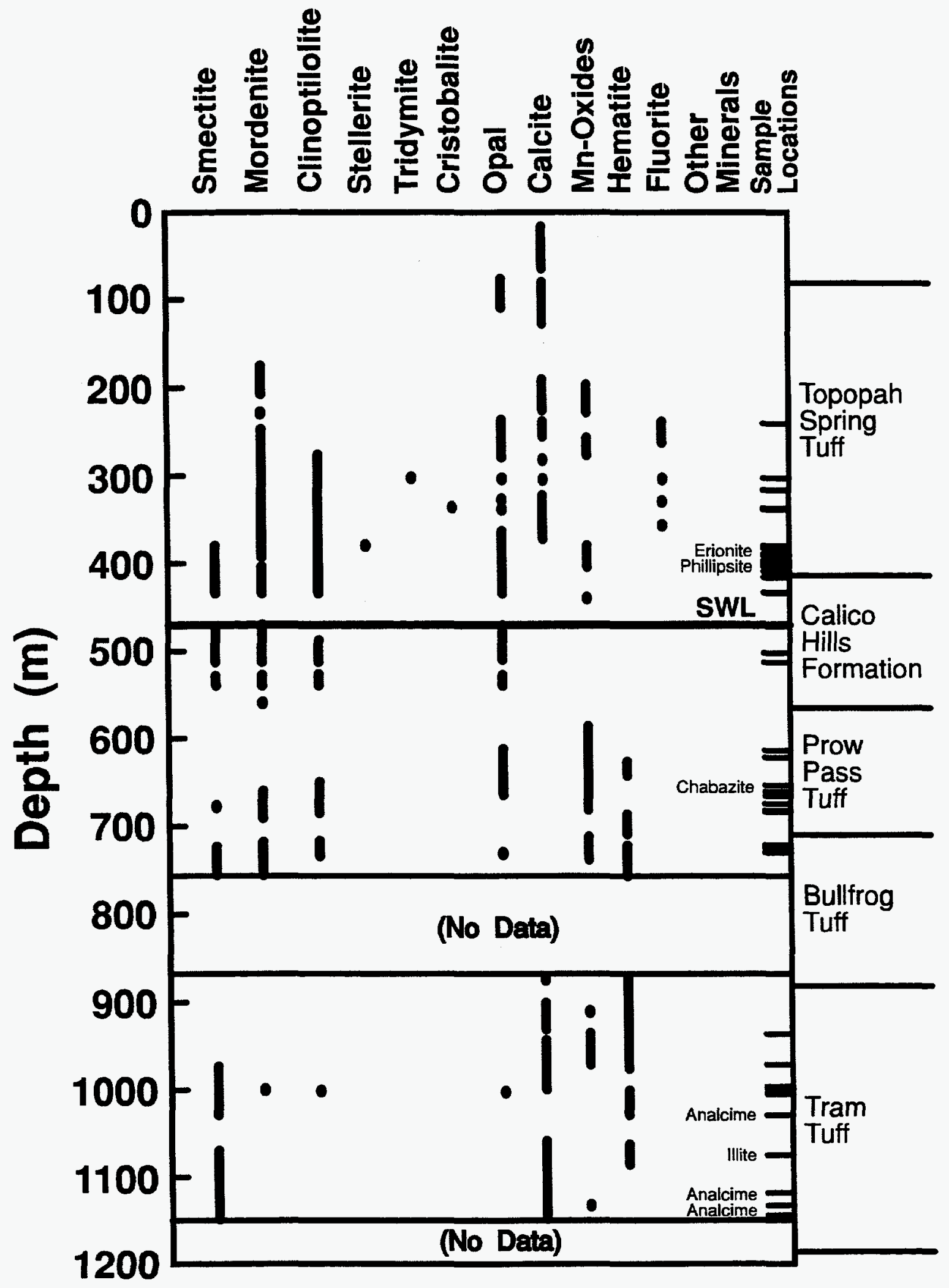

Figure 3a. Distribution of fracture-lining minerals in drill cores UE-25a\#1 and UE-25b\#1. 


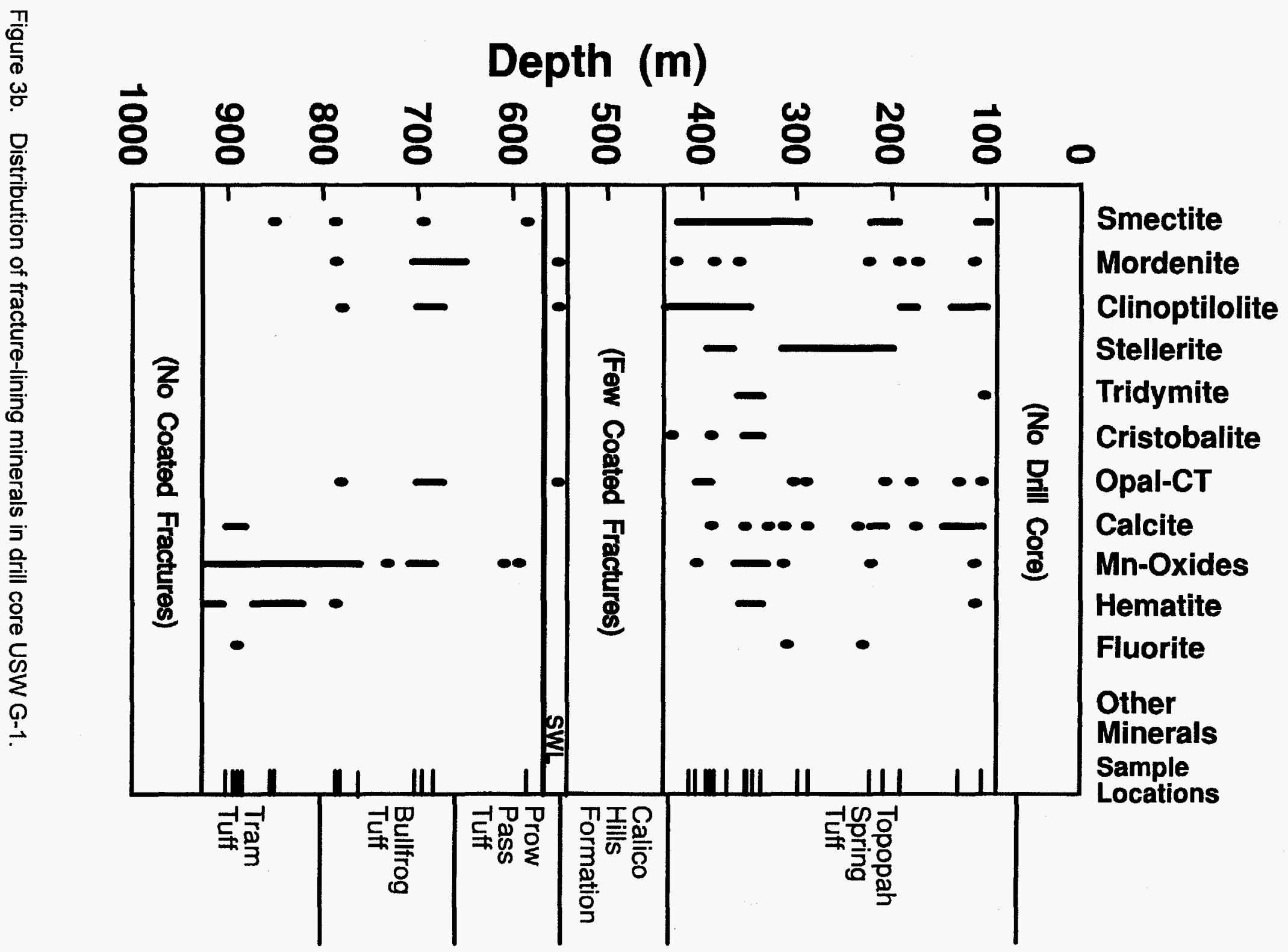




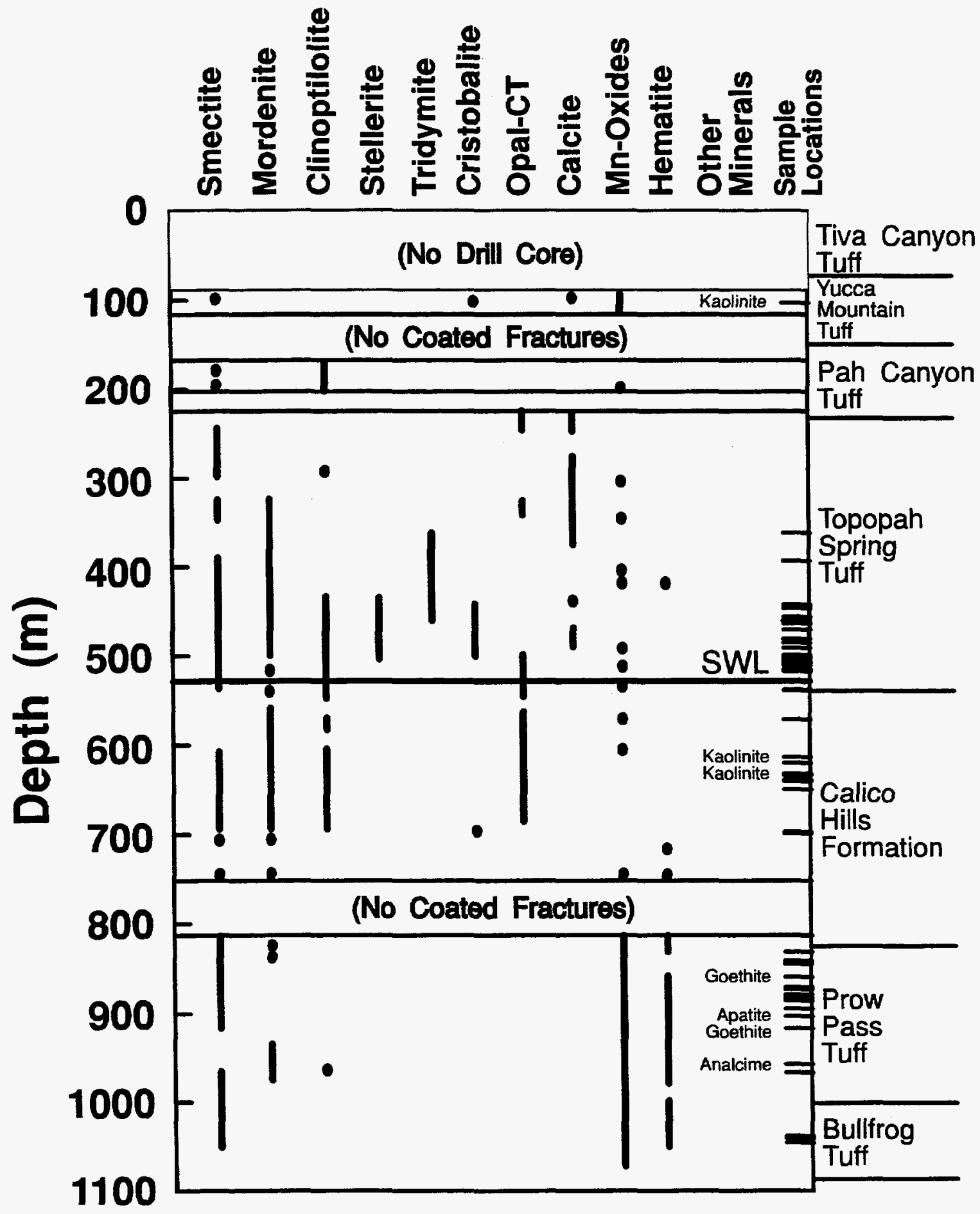

Figure 3c. Distribution of fracture-lining minerals in drill core USW G-2. 


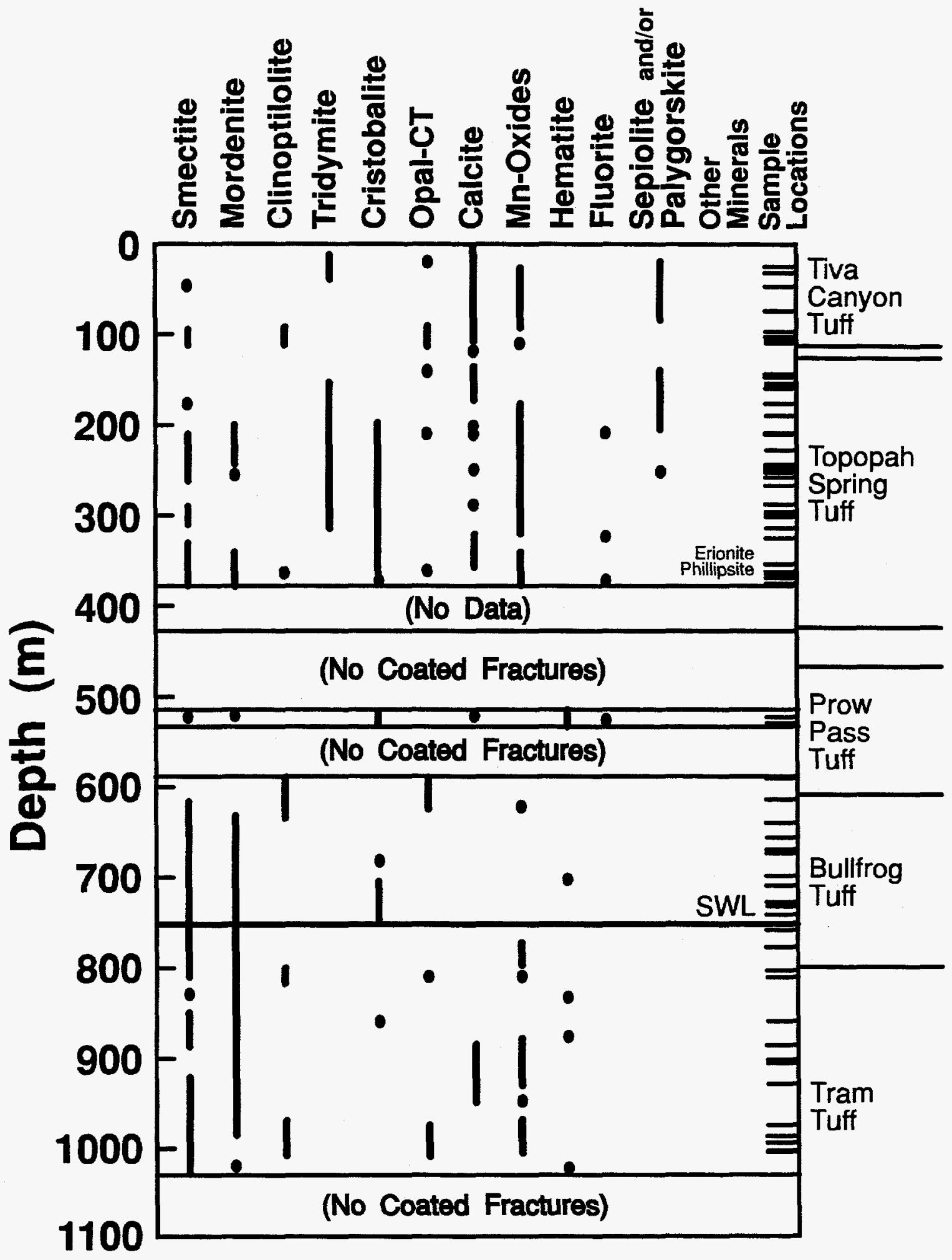

Figure 3d. Distribution of fracture-lining minerals in drill cores USW GU-3 and USW G-3. 


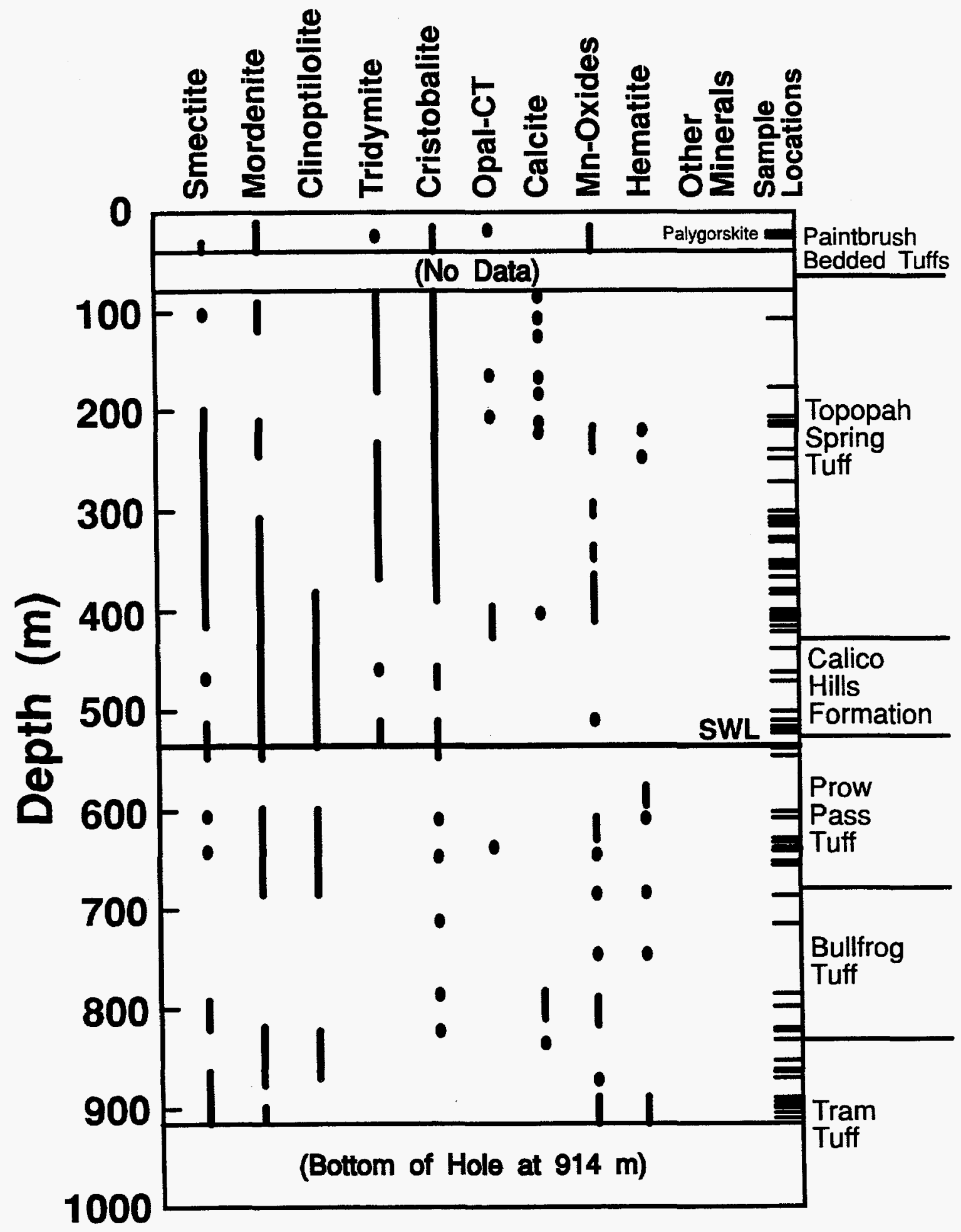

Figure 3e. Distribution of fracture-lining minerals in drill core USW G-4. 
smectite occur sporadically in fractures in the Paintbrush Group in drill hole USW GU-3, either in varying combinations of the three minerals or singularly. Several occurrences of illite have been identified in the deeper tuffs.

The Zone I-II boundary is not coincident with the base of the Paintbrush Group, but as the nonwelded vitric tuff in the Calico Hills Formation supports few fractures, samples from Zone $I$ included in this study are limited to the Paintbrush Group. Fracture-lining zeolites below the Paintbrush Group occur principally in the zeolitic intervals.

\section{Zone 1. Paintbrush Group}

The Paintbrush Group consists of four formations (Figure 2). The Tiva Canyon Tuff and Topopah Spring Tuff are primarily densely welded, devitrified tuffs containing intervals of vaporphase alteration and lithophysal cavities that formed during the early cooling and consolidation of the tuff. Thin vitrophyres are present in the Tiva Canyon Tuff and near the top of the Topopah Spring Tuff, and a 15- to 20-m-thick (basal) vitrophyre occurs near the base of the Topopah Spring Tuff. The Pah Canyon and Yucca Mountain Tuffs of the Paintbrush Group are generally nonwelded and support few fractures. Only in USW G-2, where these units are thicker, were fractures examined. The abundances and types of fractures in the Paintbrush Group are controlled by the extent of welding and devitrification in the tuff and by the abundance of lithophysal cavities. The distribution of fracture-lining minerals varies both laterally and vertically within the Paintbrush Group across Yucca Mountain.

\section{Devitrified Tuffs within the Paintbrush Group}

In the densely welded devitrified portions of the Paintbrush Group, the zeolite minerals mordenite, heulandite, and stellerite are widespread in fractures even though the tuff matrix is generally nonzeolitic. Heulandite and stellerite occur as small (10- to 50- $\mathrm{mm}$ long) prismatic crystals on fractures throughout the devitrified intervals of the Topopah Spring Tuff in at least one drill core (USW G-1) and in more limited distribution in other drill cores. Although peak overlaps with heulandite, mordenite, cristobalite, and feldspar make XRD identification of minor amounts $(<10 \%)$ of stellerite difficult, heulandite and stellerite can be distinguished in SEM images by the pinacoidal terminations of stellerite (Figure 4) and the pyramidal terminations of heulandite (Figure 5) and by EDX analyses because $\mathrm{Ca}$ is the major exchangeable cation in stellerite, whereas heulandite contains $\mathrm{Mg}$ and minor amounts of $\mathrm{Na}$ and $\mathrm{K}$ in addition to $\mathrm{Ca}$. Heulandite and stellerite may coexist within a fracture or occur separately at different intervals 


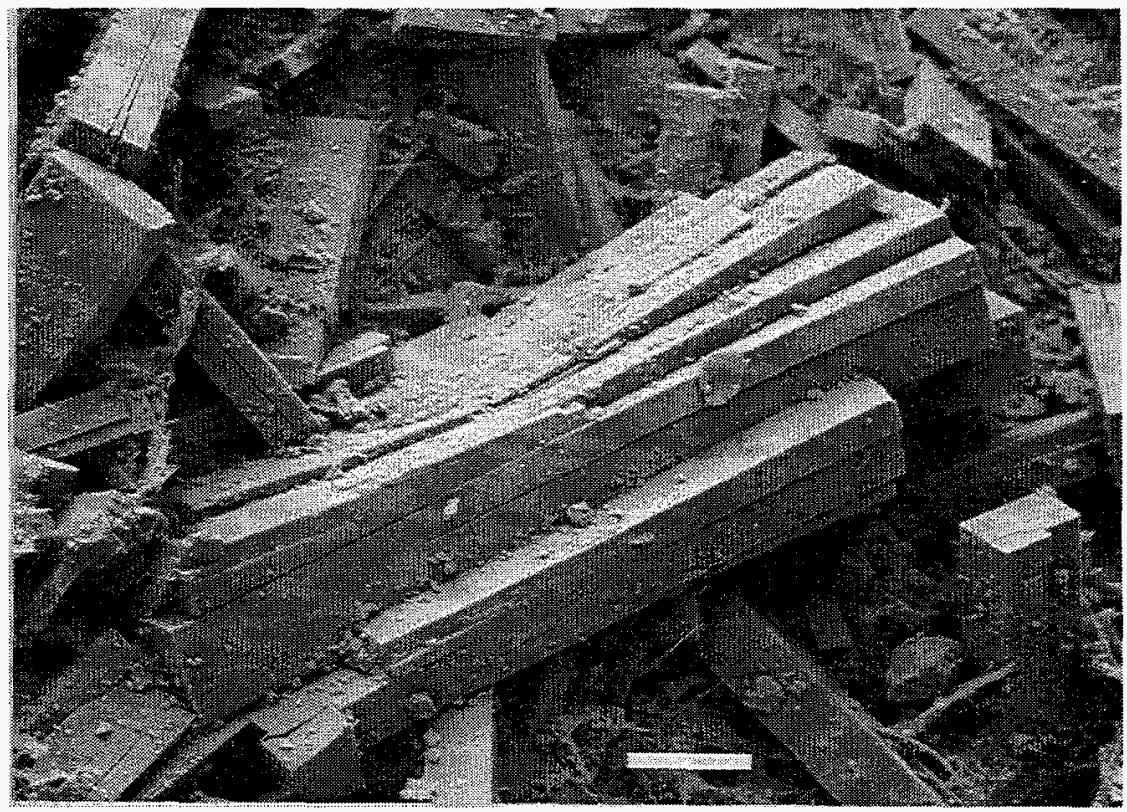

Figure 4. Secondary-electron image of stellerite crystals from sample USW G-2 1536. Scale bar is $50 \mu \mathrm{m}$.

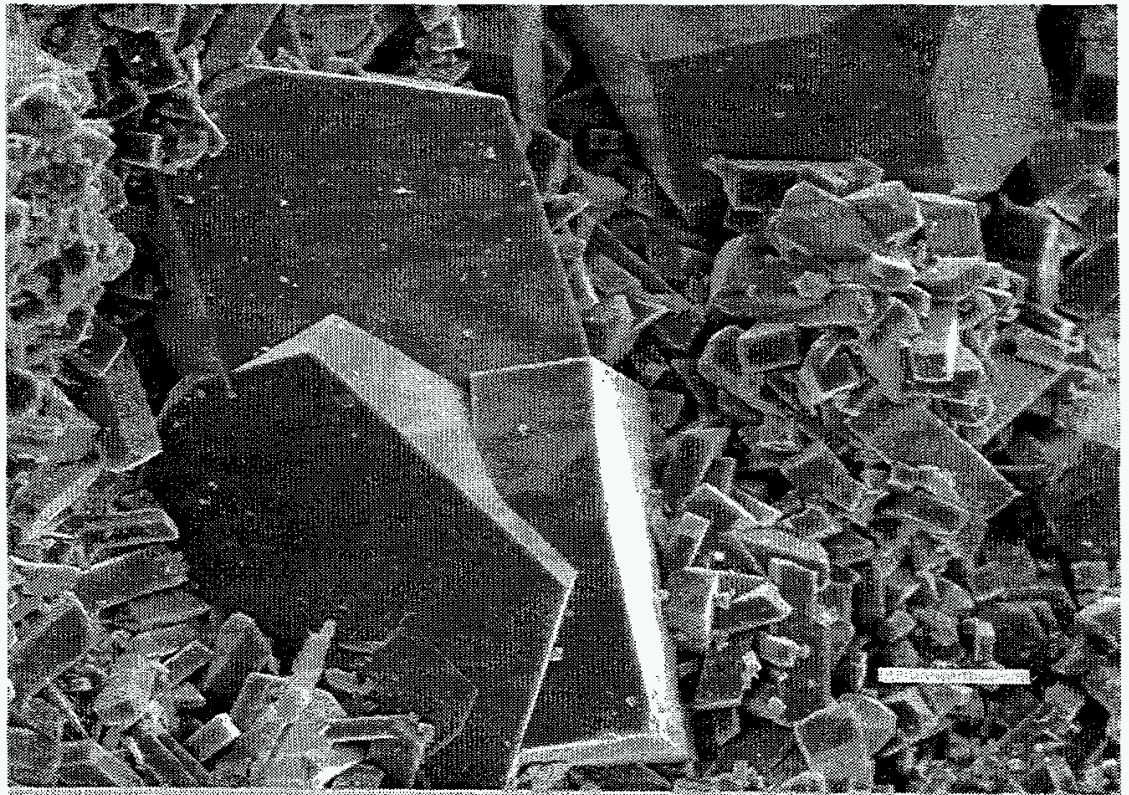

Figure 5. Secondary-electron image of large tabular and small prismatic crystals of heulandite from sample UE-25a\#1 1243. Scale bar is $50 \mu \mathrm{m}$. 
within a drill core. Stellerite occurs in fractures throughout much of the Topopah Spring Tuff in drill hole USW G-1, but it is much less abundant than heulandite in drill hole UE-25a\#1. Heulandite and stellerite both have more limited distribution in drill hole USW G-2, the northernmost hole, in which they occur alone or together in fractures and lithophysal cavities. Stellerite has not been identified in drill hole USW GU-3, the southernmost hole, nor in drill hole USW G-4.

Large $(50$ to $300 \mu \mathrm{m})$ tabular heulandite crystals (Figure 5) occur with prismatic heulandite (and locally with stellerite) in an interval extending 10 to $15 \mathrm{~m}$ above the top of the basal vitrophyre of the Topopah Spring Tuff in most drill cores. The exception to this occurrence is USW GU-3, where this interval with tabular heulandite crystals is only $1 \mathrm{~m}$ thick above the basal vitrophyre. This is the only interval in the Topopah Spring Tuff in drill hole USW GU-3 that contains heulandite, although heulandite is present in the lower part of the Tiva Canyon Tuff in this drill hole. This zone of two heulandite morphologies in fractures generally coincides with a thin zeolitic/argillic interval in the tuff immediately above the vitrophyre.

Chemical analyses of heulandite, of heulandite coexisting with stellerite, and of coexisting tabular and prismatic heulandite are presented in Appendix II. For coexisting tabular and prismatic heulandites in the same fracture, the exchangeable-cation analyses of the prismatic heulandite cluster tightly and are significantly higher in $\mathrm{Mg}$ and lower in $\mathrm{Na}$ and $\mathrm{Sr}$ than those of the tabular heulandite (for example, sample UE-25a\#1 1242 in Figures 6a and 6b). In addition, some tabular crystals are zoned, with Sr-rich cores and Mg-rich rims. Stellerite is not plotted on Figures $6 \mathrm{a}$ and $6 \mathrm{~b}$ because it deviates little from the calcium endmember (Appendix II); the structure of stellerite does not accommodate significant substitution of other extra-framework cations (Gottardi and Galli, 1985).

Mordenite occurs as discontinuous blue-white crusts of very fine grained ( $<1 \mu \mathrm{m}$ long) crystals, particularly in the shallower intervals and on smooth planar and curviplanar cooling fractures. It also occurs as mats of longer crystals (as long as $100 \mu \mathrm{m}$ ) partially covering stellerite and/or heulandite, generally on rougher fractures and locally in lithophysal cavities. Although widely distributed in fractures across Yucca Mountain, mordenite is not equally abundant in all drill holes nor at all depths in the Paintbrush Group. Small amounts of mordenite occur as crusts in the devitrified Tiva Canyon Tuff. In the densely welded devitrified Topopah Spring Tuff mordenite crusts increase in size and abundance with depth within each drill hole from about $1 \%$ of the fracture surface to more than $20 \%$ (more than $50 \%$ in some 


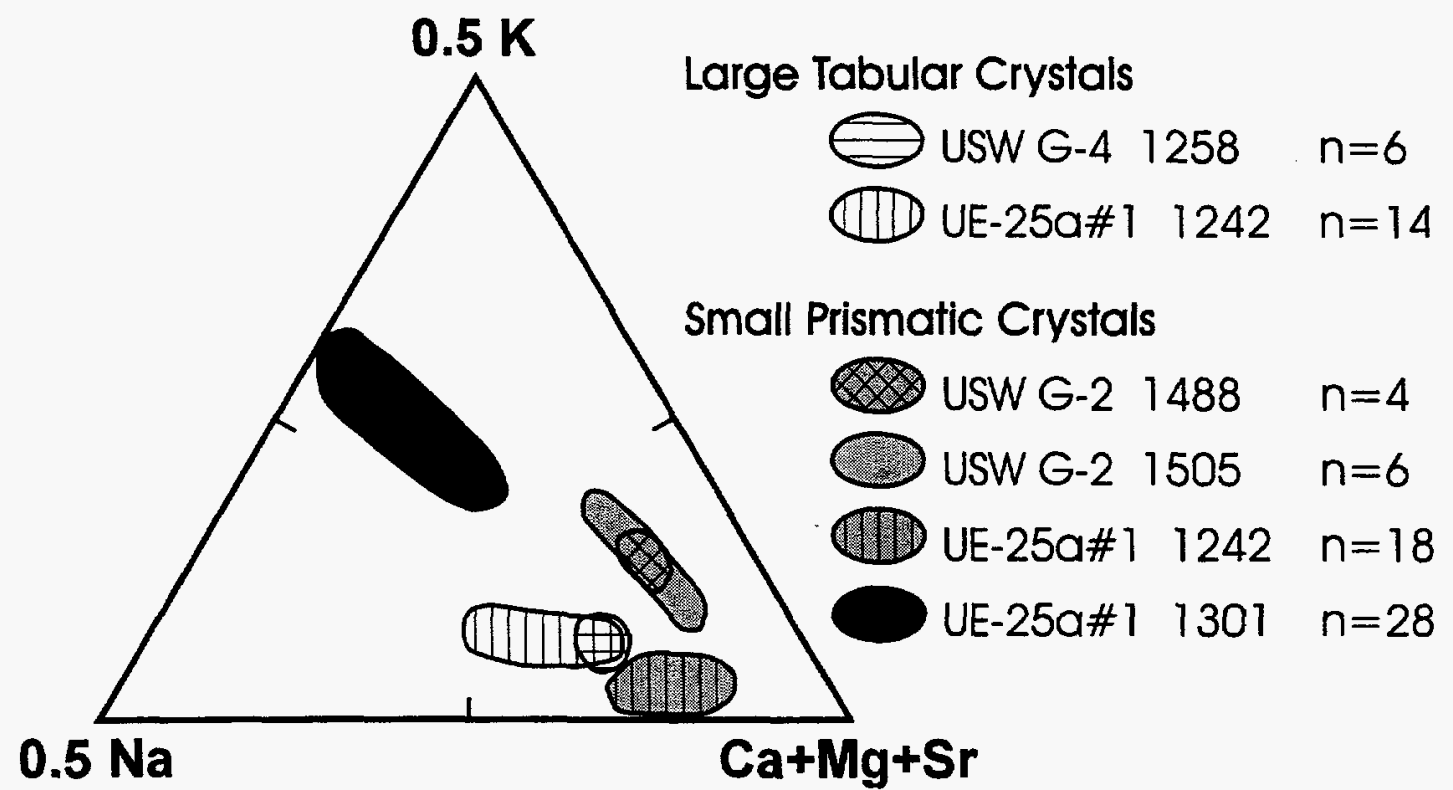

Figure 6a. Ternary plot of exchangeable cations showing chemical variability of heulandite in fractures in the Topopah Spring Tuff of the Paintbrush Group. (Data from Appendix II). Note the different trends for large tabular vs. small prismatic crystals. Heulandite from the basal vitrophyre of the Topopah Spring Tuff (UE-25a\#1 1301) forms a separate group. The number of analyses included in each group is indicated by $n$.

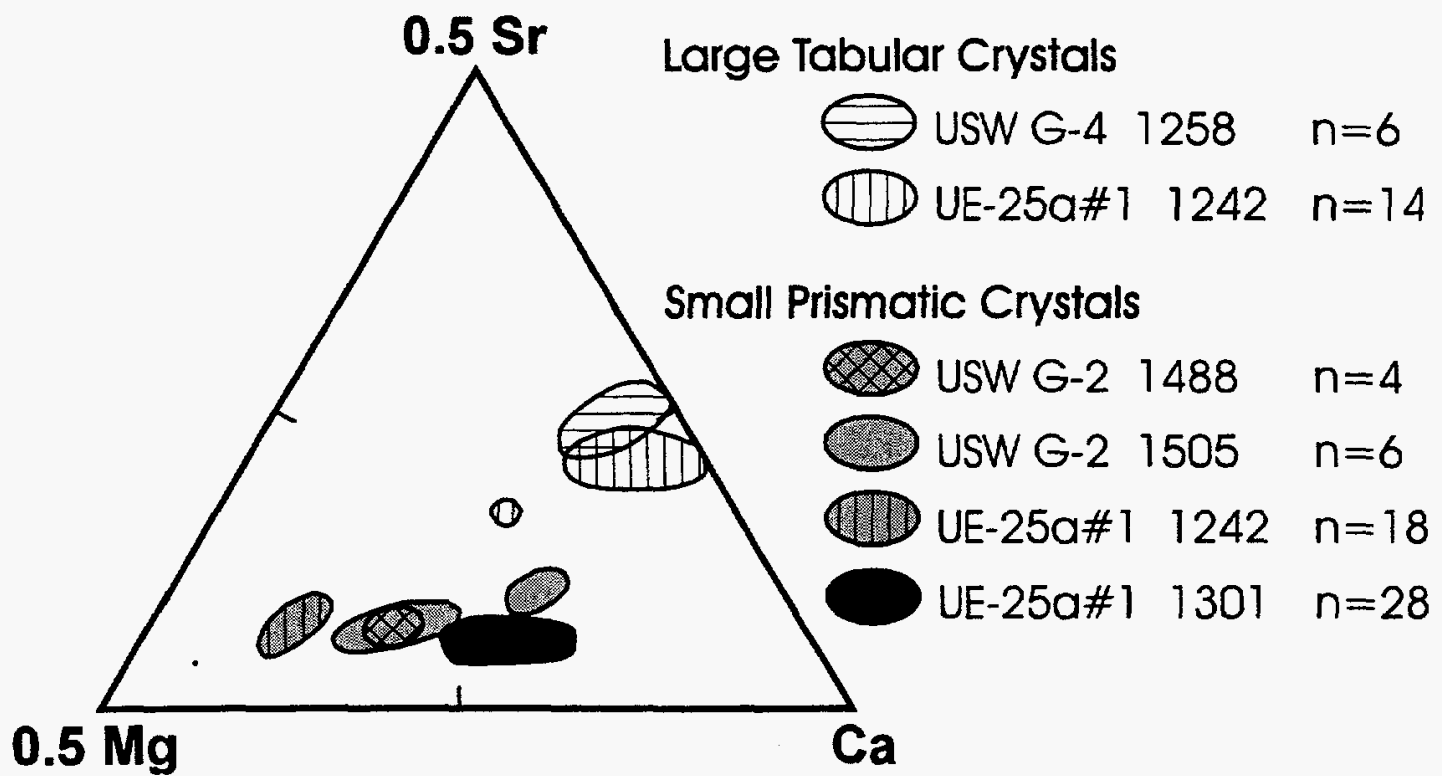

Figure 6b. Ternary plot of $\mathrm{Ca}, \mathrm{Mg}$, and $\mathrm{Sr}$ in the heulandite samples plotted in Figure $6 \mathrm{a}$. This plot highlights the difference between large tabular crystals (which have two compositional clusters in sample UE-25a\#1 1242) and coexisting small, prismatic crystals in the same fracture. 
holes). Existing data do not indicate any trend in the lateral distribution of mordenite. Trace amounts of mordenite too minor to be detected by XRD analysis were often identifiable in SEM images. Mordenite was not chemically analyzed because of the small crystal sizes and the difficulty in obtaining pure separates.

Lithophysal cavities and many fractures in and near lithophysal intervals contain tridymite and/or quartz crystals. These cavities and fractures are often rimmed with lightercolored zones of vapor-phase alteration. The tridymite morphology of the coatings has been retained (Figure 7) although the tridymite is often partially or wholly replaced by quartz or cristobalite. Euhedral quartz crystals occur in lithophysal cavities with or instead of tridymite and are also common in fractures unrelated to lithophysae. Some lithophysal cavities, particularly those in the Topopah Spring Tuff, contain calcite over tridymite and may also contain fluorite and/or opal. The most common depositional sequence is tridymite, fluorite, calcite, and finally opal, although calcite may also occur beneath the fluorite or over the opal. Both calcite and fluorite are most abundant in USW GU-3 near the southern end of Yucca Mountain and are somewhat less widespread in UE-25a\#1, near the northern end of Yucca Mountain. Fluorite is not abundant in other drill holes. Opal coats fractures in the Tiva Canyon Tuff and in the upper Topopah Spring Tuff of the Paintbrush Group; in the lower parts of the Topopah Spring Tuff some opal has recrystallized to cristobalite. Cristobalite often occurs as small acicular crystals, especially in zeolitic intervals.

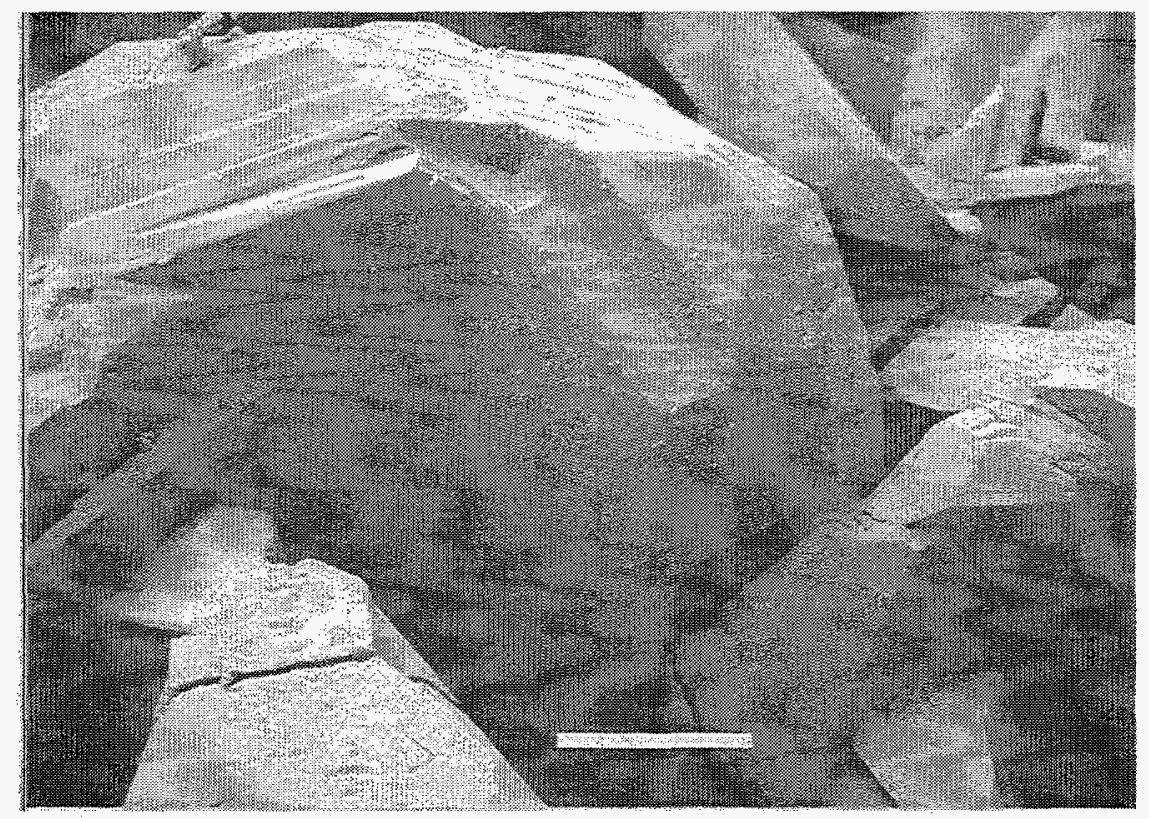

Figure 7. Secondary-electron image of tridymite morphology in lithophysal coating from sample USW G-2 1178. Mineral is now mostly quartz. Scale bar is $100 \mu \mathrm{m}$. 
Calcite occurs commonly in lithophysal cavities but also fills fractures that are not visibly connected with lithophysal cavities. Calcite-filled fractures are abundant in the Tiva Canyon Tuff in both UE-25a\#1 and USW GU-3 and in the upper part of the Topopah Spring Tuff in all drill cores. In USW G-1, where calcite occurs with prismatic zeolites, the calcite is the youngest coating in the fracture.

In drill core USW GU-3, smectite and the chain-structure clays sepiolite and palygorskite form white and yellow crusts on fractures in the Tiva Canyon Tuff and in the upper Topopah Spring Tuff, commonly over manganese-oxide minerals. Palygorskite may occur in the same fracture with sepiolite or smectite, or any of the clays may occur singly. As it is not possible to distinguish the clays visually, it is not known whether they are intergrown or form separate crusts on the fracture surfaces. There is no apparent sequence of clay deposition with depth, except that palygorskite and sepiolite do not occur below about $260 \mathrm{~m}$ depth. Smectite also occurs, particularly in the lower Topopah Spring Tuff, as individual plates as much as $10 \mu \mathrm{m}$ in diameter or as clusters of plates. It may occur alone, beneath zeolites, or as overgrowths on zeolites (Figure 8 ). Kaolinite is much less common but has been identified by XRD from fractures in two drill cores (USW G-1 and G-2), where it occurs with smectite.

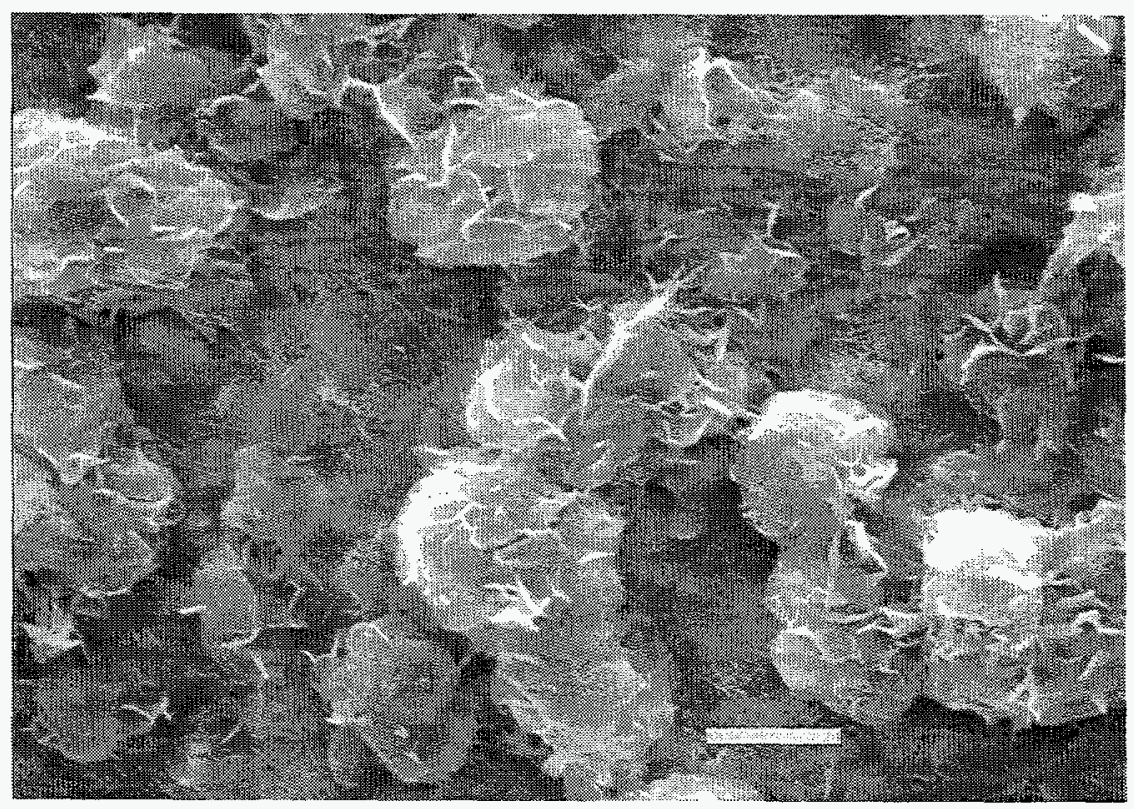

Figure 8. Secondary-electron image of smectite over mordenite from sample USW G-11156. Scale bar is $5 \mu \mathrm{m}$. 
The layer-structure manganese oxides lithiophorite and rancieite form spots and crusts ( 0.5 to $2 \mathrm{~mm}$ in diameter) and dendrites ( 1 to $2 \mathrm{~cm}$ in diameter) in the Paintbrush Group, both in densely welded devitrified intervals and in the partially welded Yucca Mountain Tuff. They are not common in lithophysal intervals. Although crusts and spots may be either rancieite or lithiophorite (as identified by XRD), dendrites are predominantly rancieite and are commonly banded with increased amounts of $\mathrm{Ce}$ and $\mathrm{Pb}$, alternating with increased $\mathrm{Al}$ content (Figures $9 \mathrm{a}$ and $9 \mathrm{~b})$. Appendix III contains electron microbeam analyses of rancieite and lithiophorite; the chemistry of these minerals is discussed in more detail in Carlos et al. (1993).

\section{Basal Vitrophyre within the Topopah Spring Tuff}

Minerals lining fractures within the basal vitrophyre of the Topopah Spring Tuff are extremely fine grained and were identified by XRD. The most abundant fracture coatings are smectite, opal-CT, and manganese-oxide dendrites, although fractures may also contain heulandite or mordenite. Phillipsite coexists with smectite in isolated occurrences in fractures within the basal vitrophyre from two drill holes (USW GU-3 and UE-25a\#1). Crystals as large as $125 \mu \mathrm{m}$ in diameter from sample USW GU-3 1200 were identified by XRD as phillipsite and were analyzed by electron microprobe. A representative analysis of these crystals is included in Appendix II. As in some other zeolites and authigenic feldspars at Yucca Mountain (Broxton et al., 1987), the Si:Al ratio obtained for this sample is higher than in the ideal formula. Erionite occurs in fractures and rock matrix in very limited intervals within the vitrophyre and in the altered tuff immediately above the basal vitrophyre in several drill holes where it comprises $<1$ $w t \%$ of the matrix but may be as much as $45 w t \%$ of the fracture coating within single fractures (Chipera and Bish, 1989; Bish and Chipera, 1991). Erionite has recently been identified in significant abundances in a 3-m-thick zone in the vitric tuff matrix immediately below the basal vitrophyre in drill hole USW UZ-14 (up to 35 wt\%; Guthrie et al., 1995). In most samples, finegrained erionite fibers ( $<30 \mu \mathrm{m}$ long) closely resemble mordenite and cannot be distinguished in hand-samples or in SEM images. Immediately above the vitrophyre in USW GU-3, the small acicular crystals visible at $25 \times$ magnification are probably erionite, although a mineral separate could not be obtained to verify this identification. The occurrence of both erionite and phillipsite is limited to an interval of a few meters in or near the upper surface of the basal vitrophyre in all of the pre-1984 cores in which they have been identified. In addition to erionite, sample USW GU-3 1189 contains a phase that has been tentatively identified as kenyaite, a layered hydrous sodium silicate found in Lake Magadi in southern Kenya (Eugster, 1967). 


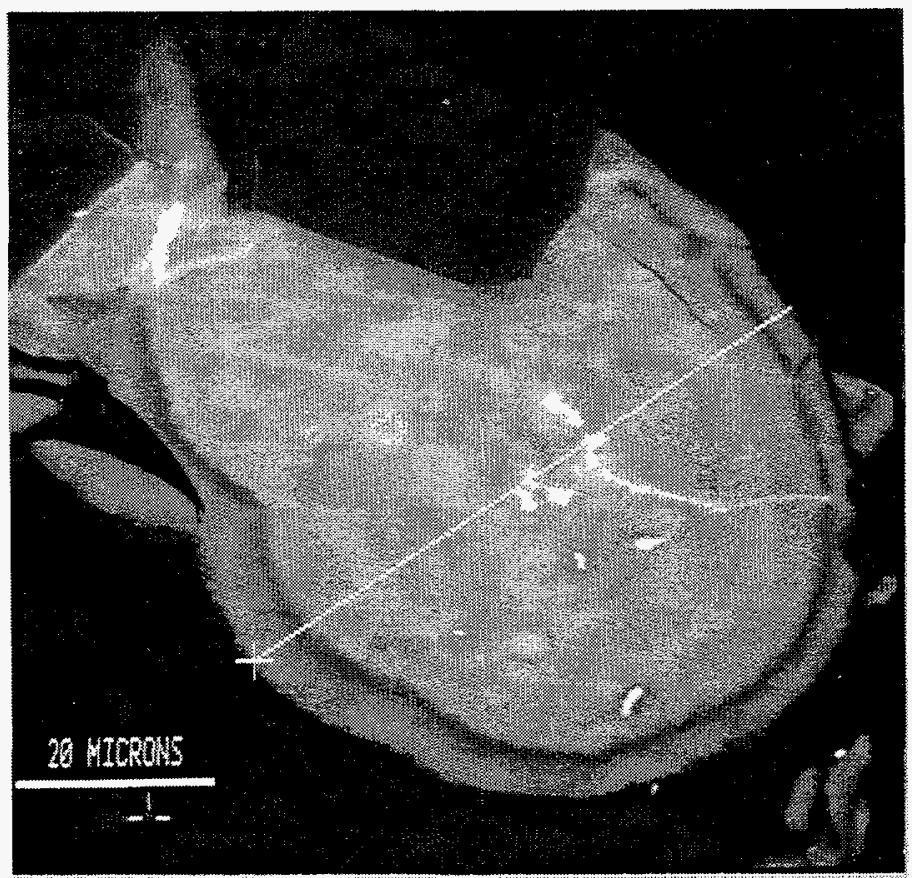

Figure 9a. Backscattered-electron image of rancieite dendrite from sample USW G-4 64 .

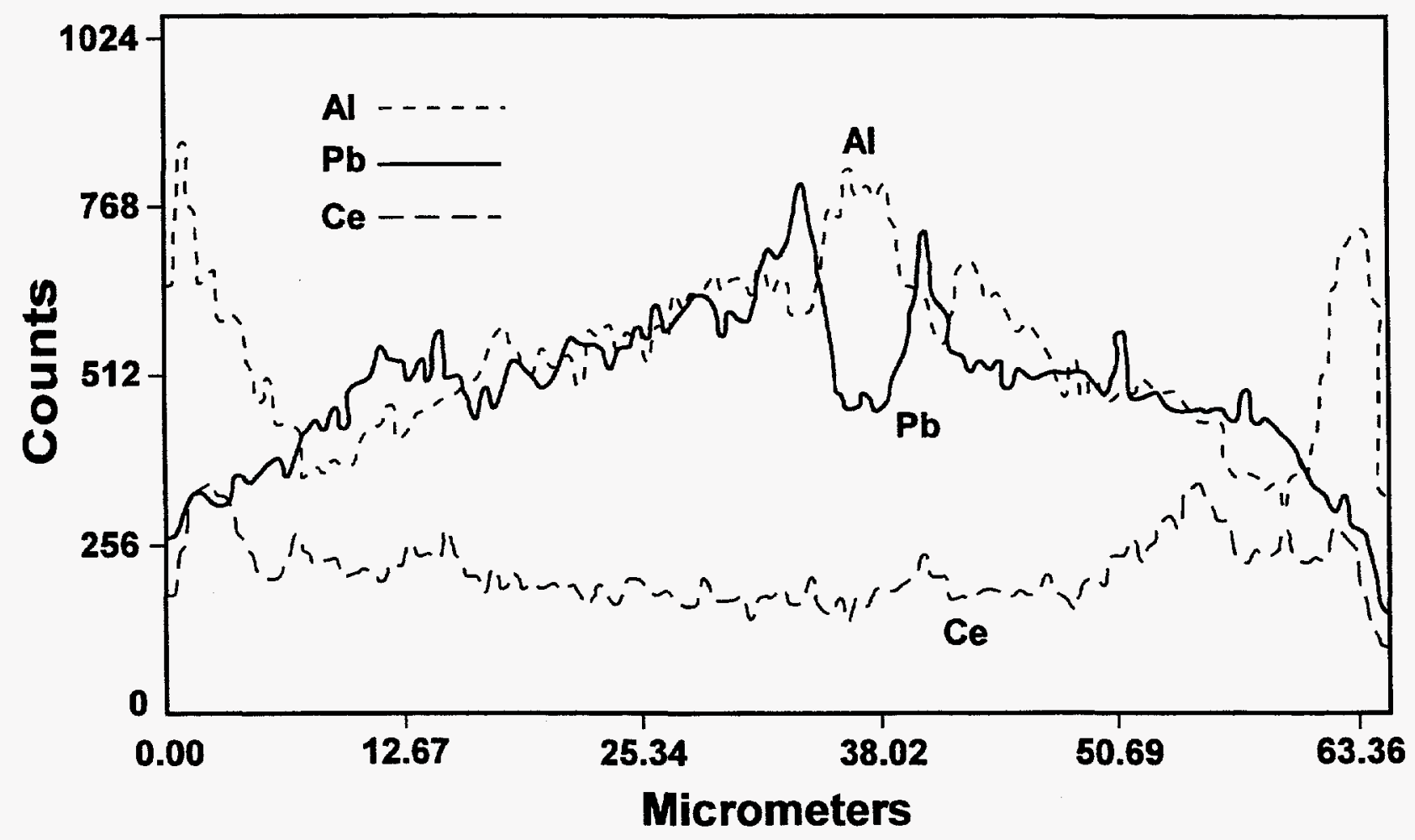

Figure 9b. Trace of $\mathrm{Al}, \mathrm{Pb}$, and $\mathrm{Ce}$ across the rancieite dendrite along the line in Figure $9 \mathrm{a}$. 
Chabazite has been identified by XRD in fracture coatings within the basal vitrophyre of the Topopah Spring Tuff in water well J-13 in Jackass Flat (Carlos, 1989) and in drill hole USW $\mathrm{VH}-1$, about $8 \mathrm{~km}$ southwest of Yucca Mountain in Crater Flat. The vitrophyre in both these holes is below the SWL. As with many zeolites lining fractures in the Topopah Spring Tuff basal vitrophyre at Yucca Mountain, cryptocrystalline coatings do not permit visual identification of minerals or of textural relationships in these samples. Chabazite has not been identified from fractures in diagenetic Zone I (above the SWL) at Yucca Mountain.

\section{Diagenetic Zones II and III}

Diagenetic Zone II includes the zeolitic Calico Hills Formation and, for most drill holes, the zeolitic portions of at least the upper tuffs of the Crater Flat Group. Unlike the matrix alteration, which is commonly dominated by clinoptilolite, mordenite and subordinate clinoptilolite are the principal fracture-lining zeolites. Mordenite forms mats and crusts of fibers on fracture surfaces, with individual mordenite crystals in the Calico Hills Formation usually from 2 to $15 \mu \mathrm{m}$ in length, locally reaching $30 \mu \mathrm{m}$ in length. Opal-CT commonly occurs interstitial to the mordenite crystals, but it also can occur as spheres as much as $10 \mu \mathrm{m}$ in diameter underlying mordenite. Mordenite forms thin discontinuous coatings or thick mats of fibers 10 to $30 \mu \mathrm{m}$ (rarely as much as $100 \mu \mathrm{m}$ ) in length in the Crater Flat Group (Figure 10).

Clinoptilolite coexists with mordenite in Zone II, which extends to the base of the Crater Flat Group in the south (in drill hole USW G-3) but includes only the upper tuffs of the Crater Flat Group in the more northern holes. Large (as long as $200 \mu \mathrm{m}$ ) prismatic or tabular crystals of clinoptilolite occur locally in fractures in the Calico Hills Formation and in the Prow Pass and Bullfrog Tuffs of the Crater Flat Group over most of Yucca Mountain, but they also occur in the Tram Tuff of the Crater Flat Group in the southernmost drill hole (USW G-3). In some fractures, small euhedral crystals of clinoptilolite ( 5 to $10 \mu \mathrm{m}$ diameter) are covered by mordenite. Clinoptilolite was commonly identified only by XRD analysis of fracture coatings and presumably is the small euhedral and anhedral plates (5 to $10 \mu \mathrm{m}$ in diameter) that are embedded in mats of mordenite seen in SEM images. Analyses of some of the larger clinoptilolite crystals from Zone II are presented in Appendix IV and are plotted in Figure 11. Although the exchangeable-cation composition varies greatly among samples, insufficient chemical data exist on fracture-lining clinoptilolites at Yucca Mountain to determine whether the compositions follow the same trends with increasing depth as do matrix clinoptilolites (Broxton et al., 1987). However, comparison of analyses in Appendix IV with analyses of matrix 


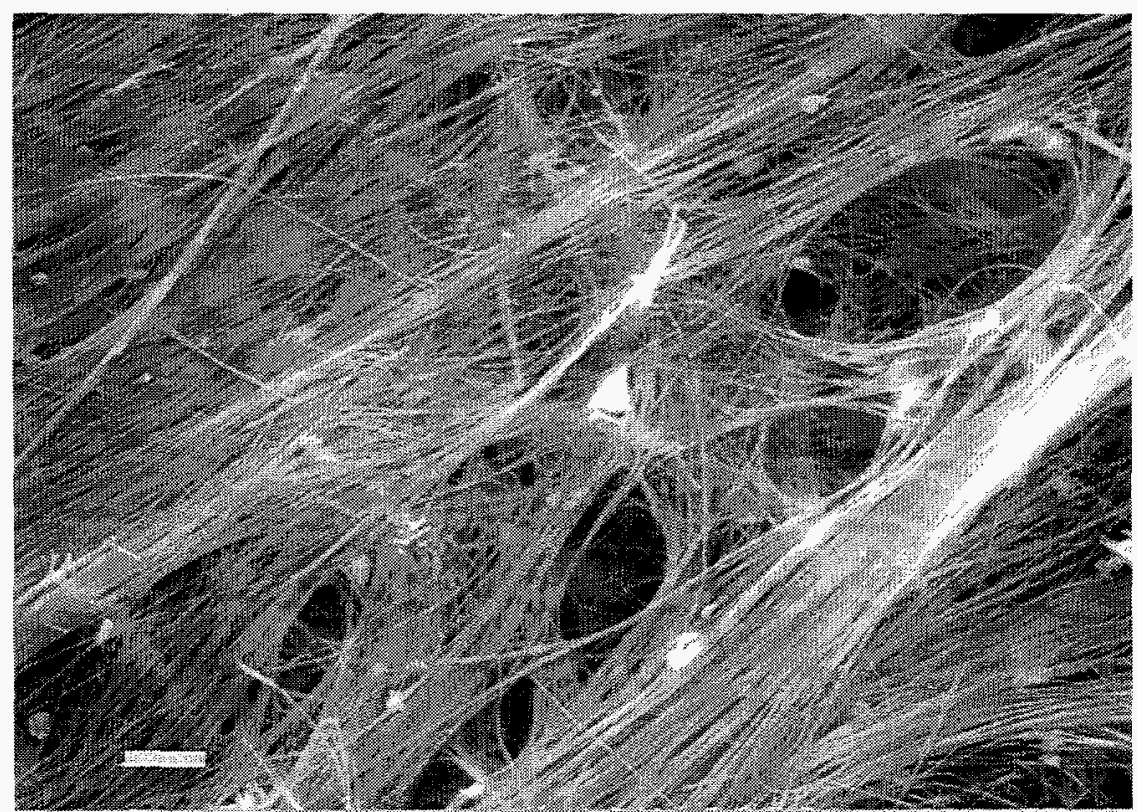

Figure 10. Secondary-electron image of mordenite from the Prow Pass Tuff of the Crater Flat Group in diagenetic Zone II. Scale bar is $10 \mu \mathrm{m}$.

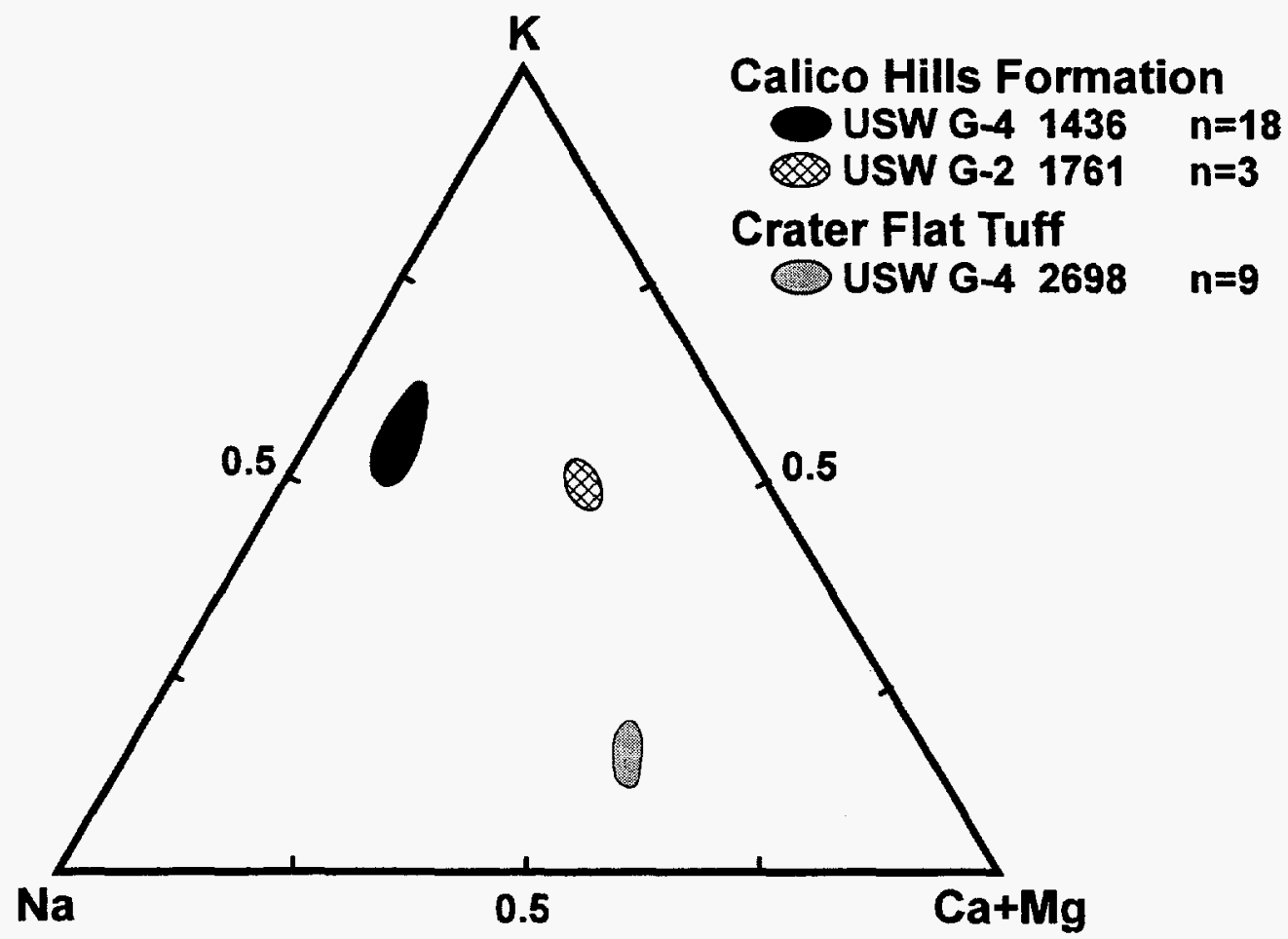

Figure 11. Ternary plot of clinoptilolite analyses from the Calico Hills Formation and the Crater Flat Group showing variation in composition among samples. The number of analyses for each sample is indicated by $n$. 
clinoptilolites from similar intervals (Broxton et al., 1986, Appendix F) shows that the fracturelining clinoptilolites are similar in composition to those in the matrix in all samples. Within diagenetic Zones II and III, the cation compositions of matrix clinoptilolites range from K-dominant to Na-dominant on the western side of Yucca Mountain and to Ca-dominant on the eastern side.

Chabazite has been identified from one sample (UE-25b\#1 2165) in the Crater Flat Group at Yucca Mountain; it coexists in Zone II with clinoptilolite and alkali feldspar (Figure 12). Chemical analyses of the chabazite are included in Appendix IV. Euhedral crystals of analcime (Figure 13) occur in fractures only at the northern end of Yucca Mountain where the Crater Flat Group is altered to diagenetic Zone III and where analcime also occurs in the matrix.

Clinoptilolite-heulandite occurs in fractures below this depth and may coexist in the matrix with analcime (Bish and Chipera, 1989). Representative analyses of analcime from a fracture in sample USW G-2 3137 are given in Appendix IV. The fracture analcime has an Si-rich, Na end-member composition, similar to the analyses of matrix analcime reported by Broxton et al. (1987). Fractures, which are less common in the most altered intervals of the Tram Tuff of the Crater Flat Group, contain no zeolites even though analcime and clinoptilolite may be present in the matrix. Hematite, calcite, and green clay (illite) are the most abundant fracture-filling minerals in the deepest portions of Zone III within the Tram Tuff.

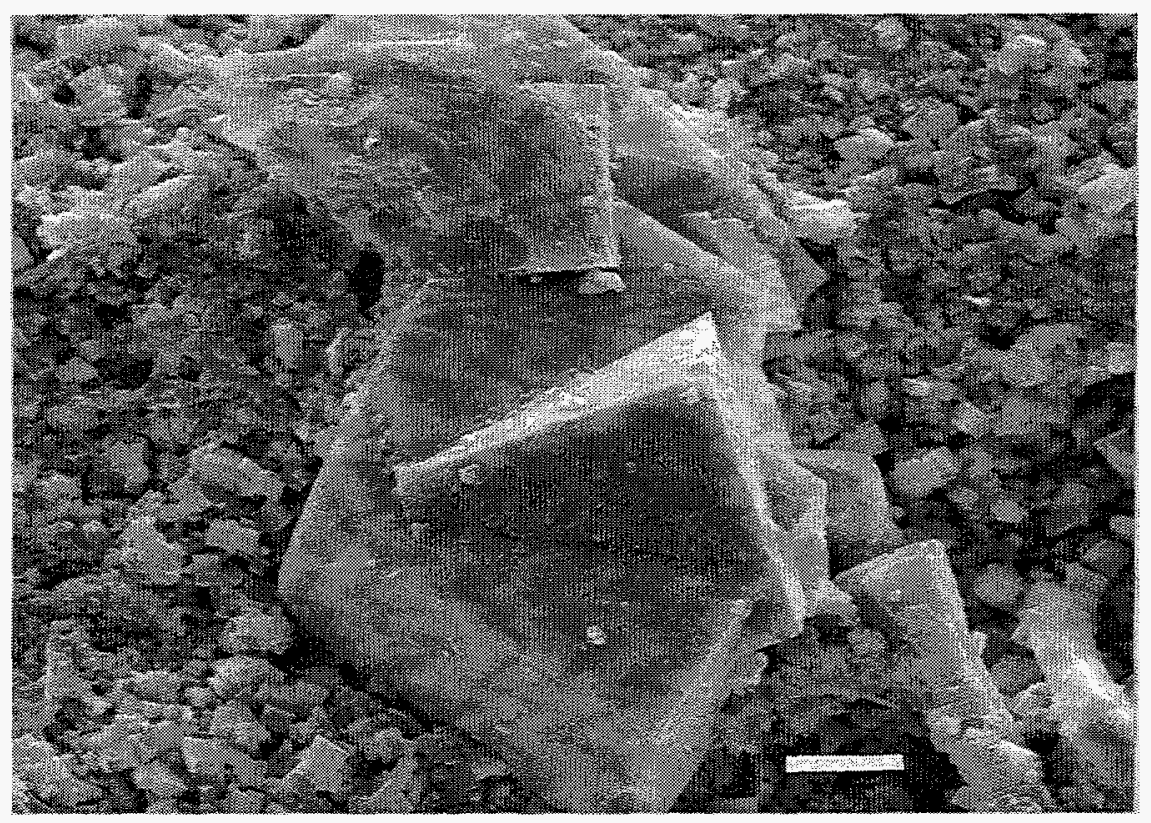

Figure 12. Secondary-electron image of chabazite from sample UE-25b\#1 2165. Scale bar is $10 \mu \mathrm{m}$. 
The central portions of the three tuffs of the Crater Flat Group are moderately to densely welded, devitrified tuff. Manganese-oxide minerals, iron oxides, quartz, and calcite are the most abundant fracture coatings in the devitrified Crater Flat Group over most of Yucca Mountain. Cryptomelane-hollandite is the most common manganese-oxide mineral throughout the Crater Flat Group below the SWL. It may occur with lithiophorite, todorokite, pyrolusite, aurorite, or, in one sample, rancieite. Hollandite surrounds large lithiophorite crystals in the Prow Pass Tuff and sometimes appears less corroded than the lithiophorite (Figure 14), suggesting that the lithiophorite was partially dissolved by later fluids, which then deposited hollandite. Hollandite also surrounds or partially replaces pyrolusite in the Crater Flat Group. Manganese-oxide coatings from several fractures in the Crater Flat Group from USW G-4 were analyzed by electron microprobe (analyses are presented in Appendix $V$ ). There appears to be a chemical continuum between cryptomelane (K-rich) and hollandite (Ba-rich). In the analyses, all $\mathrm{Mn}$ is assumed to be $\mathrm{Mn}^{4+}$, although $\mathrm{Mn}^{3+}$ is known to substitute for $\mathrm{Mn}^{4+}$ in the octahedral sites (Post et al., 1982). Most samples contain a fairly restricted range of compositions within each sample, although there is considerable chemical variation between samples. The ternary plots in Figures $15 \mathrm{a}$ and $15 \mathrm{~b}$ illustrate the most significant variations in tunnel cation chemistry; Carlos et al. (1993) contains a more detailed discussion of the manganese-oxide mineral chemistry.

In the devitrified intervals below the SWL, hematite is the dominant iron-oxide mineral. It occurs as rusty-red powder, as staining in fine-grained quartz coatings, and as intergrowths with manganese oxides. In the northernmost hole (USW G-2), goethite occurs with hematite in several fractures. Mordenite is generally the only zeolite found in fractures in the devitrified Crater Flat Group, and its distribution and abundance vary across the mountain, with the greatest abundance being in the southernmost drill holes (USW GU-3 and USW G-3). Smectite occurs with mordenite in some fractures in the devitrified intervals. Quartz is common in fractures in the devitrified intervals, usually as euhedral crystals. Lithophysal-type fracture coatings occur in the Bullfrog and Tram Tuffs of the Crater Flat Group; although the tridymite morphology has been preserved, the silica mineral is now quartz. Quartz-lined fractures often contain manganese-oxide minerals and may also contain calcite or mordenite. Fluorite occurs with quartz and calcite in one fracture in the Tram Tuff in USW G-1. Calcite is unevenly distributed but is locally abundant as a fracture filling in the Crater Flat Group. As mentioned above, it is the most common filling in the more altered tuffs of Zone III, but it also occurs over restricted depth intervals in the devitrified tuffs, usually with manganese-oxide minerals. 


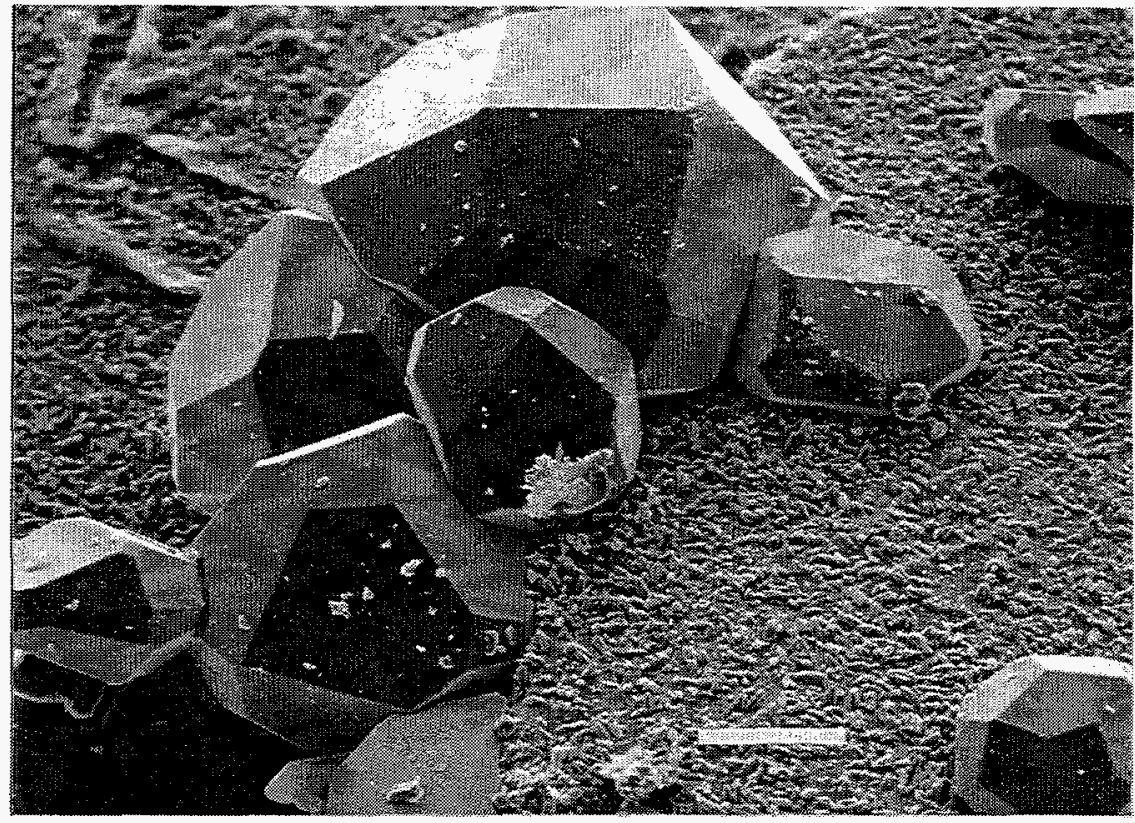

Figure 13. Secondary-electron image of analcime over quartz from sample USW G-2 3137. Mordenite is visible as the ropelike feature in the upper left corner of the photo. Scale bar is $100 \mu \mathrm{m}$.

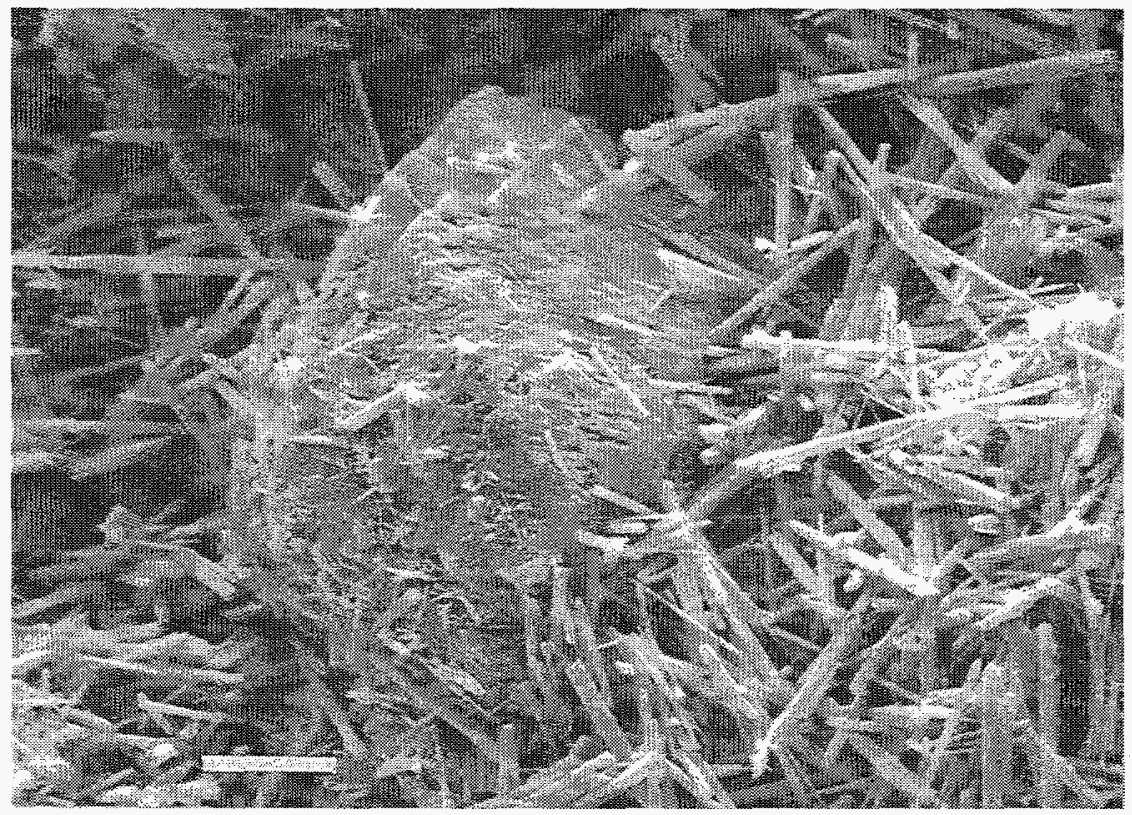

Figure 14. Secondary-electron image of large lithiophorite crystal surrounded by rods of hollandite from sample USW G-2 2878. Scale bar is $10 \mu \mathrm{m}$. 

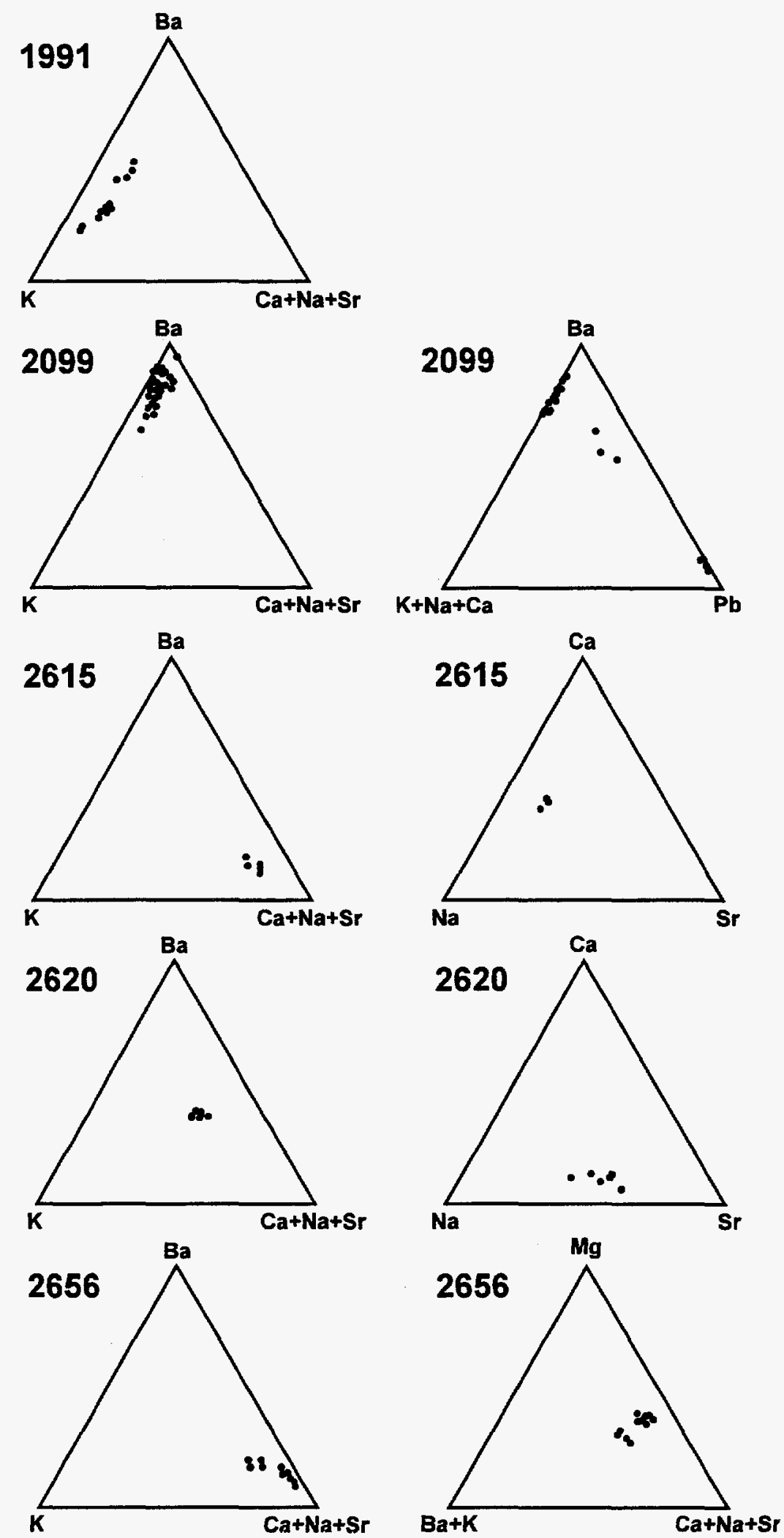

Figure 15a. Ternary plots of proportions of tunnel cations showing chemical variability in the manganese-oxide minerals in the Prow Pass and Bullfrog Tuffs of the Crater Flat Group in drill hole USW G-4. Sample numbers indicate sample depth in feet. 

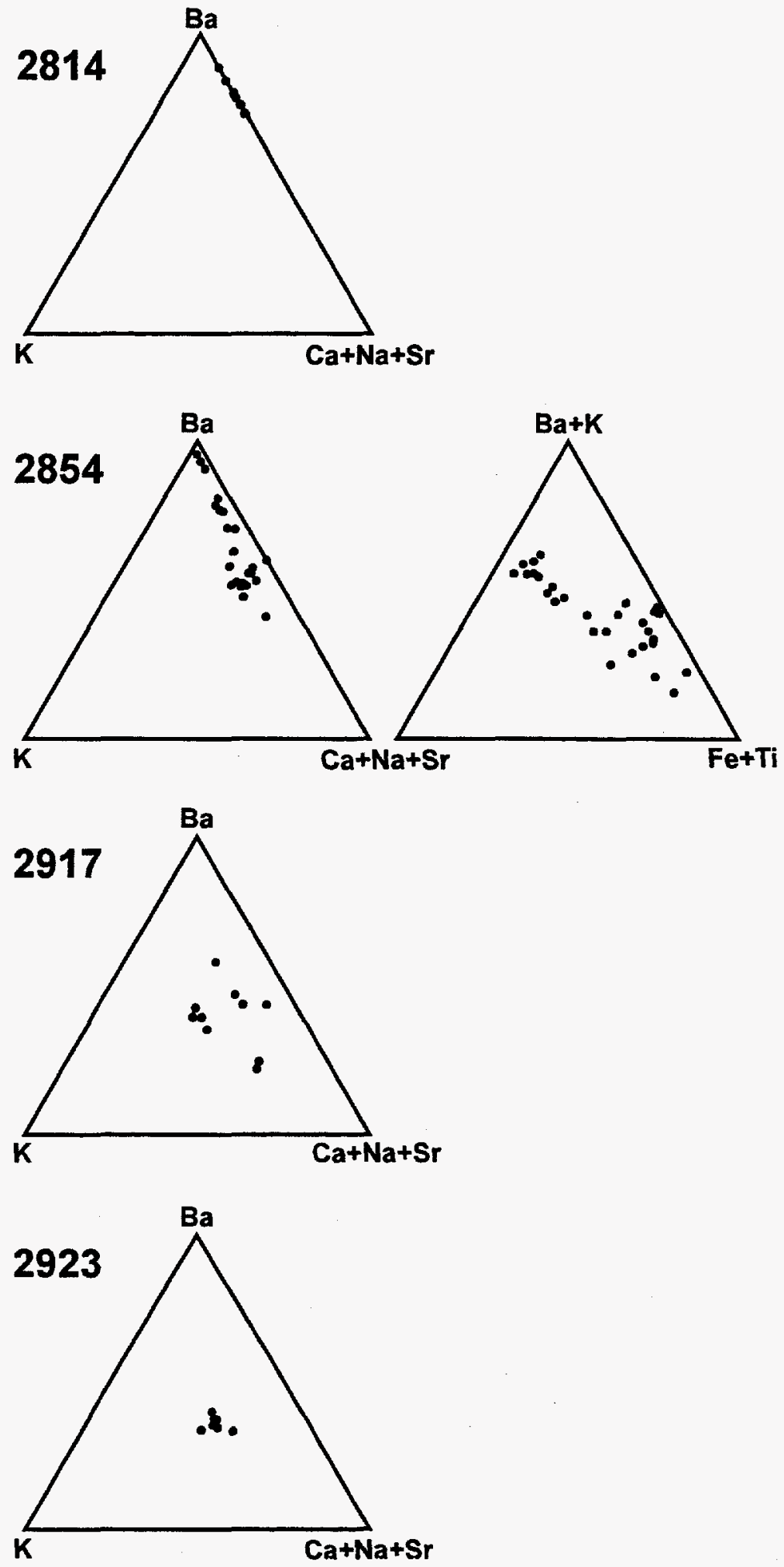
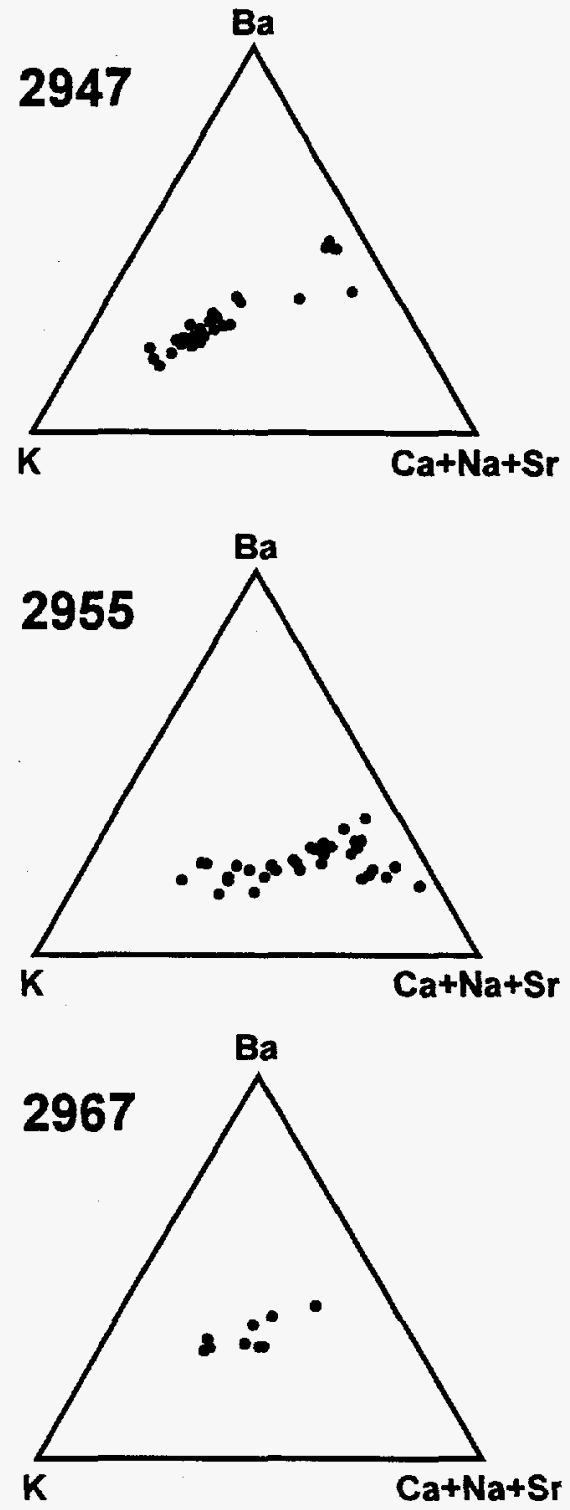

Figure 15b. Ternary plots of proportions of tunnel cations showing chemical variability in the manganese-oxide mineral in the Tram Tuff of the Crater Flat Group in drill hole USW G-4. Sample numbers indicate sample depth in feet. 


\section{DISCUSSION}

\section{Distribution and Paragenesis}

The distribution of zeolite minerals in fractures at Yucca Mountain appears to depend on several factors but generally correlates with the diagenetic alteration zones as defined by the matrix mineralogy (Broxton et al., 1987). Zone I contains a complex and diverse suite of zeolites in fractures even though the matrix is predominantly nonzeolitic. Stellerite has been identified only from the Paintbrush Group, but it is not present in all the cores nor does it appear to be limited to one interval within the devitrified Paintbrush Group. In addition, the matrix that hosts the stellerite-containing fractures usually does not contain stellerite. Until recent analyses of samples from drill core UE-25 UZ\#16 were performed (Chipera et al., 1995b), stellerite had not been identified in any bulk-rock samples from Yucca Mountain.

The prismatic zeolites stellerite and heulandite are most widely distributed in fractures in drill holes USW G-1 and UE-25a\#1, located along Drill Hole Wash, suggesting a relationship between proximity to the wash and abundance of heulandite and stellerite. Preliminary examination of more recently drilled cores (USW UZ-14 and USW NRG-7a), however, suggests a more complex control on distribution of stellerite than simple proximity to Drill Hole Wash. As future holes are drilled, it may be possible to define better the lateral distribution of these zeolites in Zone I and identify any correlations with structural features.

Fractures in the Paintbrush Group contain a generally calcic suite of minerals, including calcite, Ca-zeolites (stellerite, heulandite, and mordenite), Ca-smectite, and rancieite, along with silica polymorphs and lithiophorite. Pore-water extracts from the Paintbrush Group where it is above the SWL (Yang, 1992) have a chemistry strikingly different from the chemistry of groundwater from the Paintbrush Group below the SWL (Benson et al., 1983), with markedly increased $\mathrm{Ca}, \mathrm{Mg}, \mathrm{K}$, and $\mathrm{Sr}$ above the SWL. Few analyses of pore waters are available, however, and the compositional variability of these waters is not known. The higher $\mathrm{Ca}, \mathrm{Mg}$, and $\mathrm{Sr}$ contents of the pore waters, compared with the composition of groundwater below the SWL, do appear to be reflected in the fracture-lining mineral assemblages in the Paintbrush Group.

Chipera et al. (1995a) used representative chemical compositions and estimated thermodynamic properties to model the conditions under which the various fracture-lining zeolites at Yucca Mountain formed. Figure 16 shows the results of a calculation constrained using a representative modern Yucca Mountain water value for silica activity (obtained from Kerrisk, 1987) and a temperature of $35^{\circ} \mathrm{C}$. Increasing the temperature for the calculation to 
$125^{\circ} \mathrm{C}$ did not change the diagram significantly. The Ca-Na-K compositions of modern Yucca Mountain groundwater (Kerrisk, 1987) plot within the clinoptilolite stability field, although either a slight decrease in silica activity or an increase in Ca content would result in conditions favorable for the formation of stellerite. Chipera et al. (1995a) found it necessary to suppress the formation of stellerite to create a stability field for heulandite. However, they speculated that heulandite would have formed more readily in their models if they had included $\mathrm{Mg}$ in the calculations.

The isolated occurrences of erionite and phillipsite at the top of the Topopah Spring Tuff basal vitrophyre in drill holes USW GU-3 and UE-25a\#1 suggest the presence of a localized geochemical environment not normally found at Yucca Mountain. Chipera et al. (1995a) found,

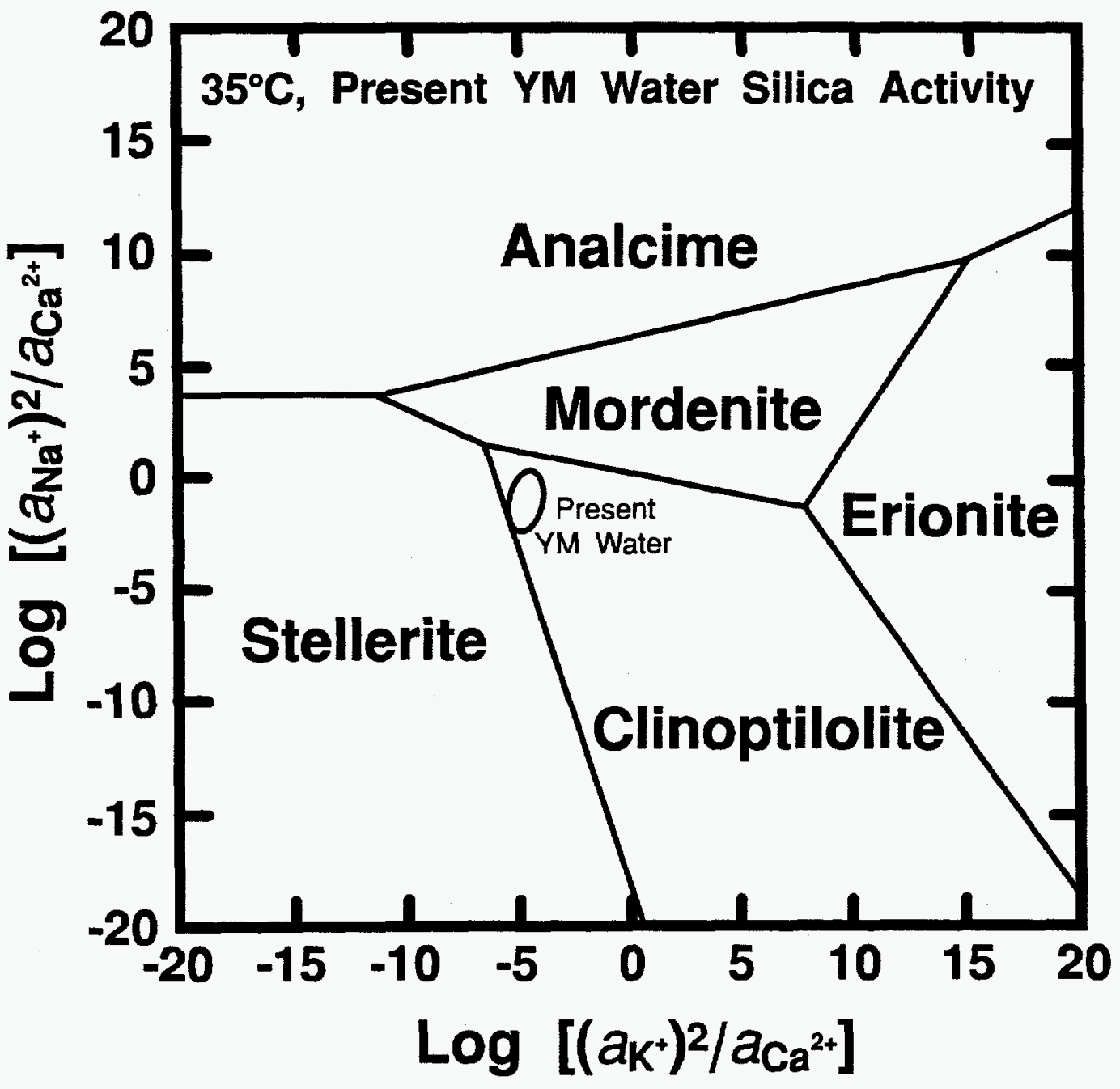

Figure 16. Log activity diagram for the zeolite phases found at Yucca Mountain calculated using estimated thermodynamic data and representative chemical analyses of the minerals (from Chipera et al., 1995a). 
in their modeling of zeolite stability, that erionite and phillipsite form under conditions with elevated $\mathrm{K}: \mathrm{Ca}$ ratios. The identification of kenyaite coexisting with erionite in USW GU-3 is tentative. Kenyaite is found in Lake Magadi in Kenya (Eugster, 1976). Lake Magadi sediments contain abundant trachytic glass that is altering primarily to erionite (Surdam and Eugster, 1976). Surdam and Eugster (1976) report water compositions for Lake Magadi that are high in alkalis and low in alkali-earths in accord with the calculations by Chipera et al. (1995a). If the identification of kenyaite is indeed correct, geochemical conditions resulting in its formation at Yucca Mountain can be constrained to $\mathrm{Na}^{+} / \mathrm{H}^{+}$ratios greater than 5 and silica activities approaching that of amorphous silica (Bricker; 1969).

The presence of two different morphologies and at least two different compositions of heulandite in fractures in the Topopah Spring Tuff suggest at least two episodes of heulandite formation. Differing compositions for the large tabular crystals within a fracture (UE-25a\#1 1242, Appendix II) suggest that different crystals formed at different times from different fluids, that later fluids resulted in partial cation exchange within the crystals, or that overgrowths of a different composition were deposited. Additional studies of individual crystals may determine whether the observed compositional variation in tabular crystals is a result of regular zoning, two or more compositions (generations) of tabular crystals and no zoning within individual crystals, cation exchange along some preferred direction within the crystals, or some combination of the above.

No direct evidence exists for the absolute age of fracture-lining zeolites in the Paintbrush Group. Heulandite in the Paintbrush Group contains insufficient $K$ to permit dating by the K-Ar technique (e.g., WoldeGabriel et al., 1993). Slickensides on crusts of fine-grained mordenite in some fractures indicate movement along the fractures after deposition of the mordenite. Other zeolites and coarser mordenite have not been affected by movement, suggesting that they may have been deposited after most of the tectonic activity at Yucca Mountain and are therefore younger than 11.6 Ma (Scott et al., 1983; Sawyer et al., 1994). Alternatively, zeolites may have been deposited during the early history of the tuff, but subsequent tectonic movement was restricted to a few fractures or zones that either did not contain coarser zeolites or the zeolites were destroyed during movement. In several fractures in the Paintbrush Group, calcite overlies coarse zeolites, and it may be possible to constrain the age of fracture-lining zeolite deposition by determining the age of the calcite.

The Zone I-Zone II boundary is neither coincident with nor parallel to the present SWL and, therefore, probably indicates the position of the SWL at the time of zeolitization, before 
tectonic tilting of Yucca Mountain and before deposition of the Rainier Mesa Tuff of the Timber Mountain Group 11.6 Ma ago (Broxton et al., 1987; Sawyer et al., 1994). Correlation of fracture-lining zeolites in diagenetic Zones II and III with degree of zeolitization of the host tuff suggests that the fracture coatings may have formed at the same time as the alteration of the tuffs. The similarity of chemical compositions of matrix and fracture-lining clinoptilolite support this theory. An increase in degree of alteration to diagenetic Zone III in both fractures and matrix toward the north of Yucca Mountain suggests that this alteration may have been a result of hydrothermal activity related to the Timber Mountain volcanism, constraining the age of the zeolites to $\geq 10.7 \mathrm{Ma}$ (Bish and Aronson, 1993). Mineralogical differences between the fracturelining zeolites and manganese-oxide minerals in the Paintbrush Group (Ca-rich) and those in the Crater Flat Group (K-, Na-rich) suggest that deposition may have occurred at different times and almost certainly from fluids of different composition.

\section{Transport}

Many fractures in Zone I that contain heulandite are not sealed and have open passages that are potential flow paths. The heulandite and mordenite coatings may act as barriers to radionuclide migration away from the potential repository, assuming that they are not affected by increased temperature after emplacement of waste (possibly up to $200^{\circ} \mathrm{C}$ in the potential repository itself; Buscheck and Nitao, 1992). Alternatively, they may react at elevated temperature with water present in the rock to form other mineral assemblages (e.g., heulandite $\Rightarrow$ analcime + calcite + silica; Bish and Aronson, 1993). Fractures that contain predominantly silica polymorphs are generally closed to present flow, but the same minerals are contained in abundant lithophysal cavities that are still open. Possible connections between these cavities cannot be determined from drill core, as the diameter of the cavities is often larger than the diameter of the core. If a repository is emplaced in Yucca Mountain, it is possible that the increased heat load may generate a convective system that could dissolve the less-stable silica polymorphs (e.g., cristobalite and tridymite) within Zone I (Buscheck and Nitao, 1992). Experimental data (Rimstidt and Newcomb, 1989) suggest that silica may be dissolved and transported in fractures away from the potential repository toward the cooler rocks where the solution would then precipitate silica and zeolites, possibly sealing the local fractures.

Inasmuch as fractures within zeolitic intervals in Zones II and III contain the same minerals as the matrix in those intervals, modeling retardation of radionuclides in solution as a function of zeolite content need only consider flow times rather than different mineralogy or 
chemistry for fractures in these intervals. Although thick mats of mordenite fibers that fill fractures will allow passage of water, they may act as filters for suspended radionuclide colloids if the colloid particle size is larger than the interstitial space between fibers $(\leq 1 \mu \mathrm{m})$.

Within the devitrified intervals in the Crater Flat Group, manganese- and iron oxideminerals are the most common coatings and are potentially the most significant minerals below the SWL for retardation of some radionuclides, particularly the actinides (Triay et al., 1991). Most of the manganese-oxide minerals in fractures below the SWL are tunnel-structure minerals (e.g., cryptomelane, hollandite, coronadite, and todorokite) that consist predominantly of $\mathrm{Mn}^{4+}$, with minor $\mathrm{Mn}^{3+}$ (Manceau and Combes, 1988; Manceau, personal communication, 1988; Post and Bish, 1988). Pyrolusite contains exclusively $\mathrm{Mn}^{4+}$, and aurorite and lithiophorite contain substantial $\mathrm{Mn}^{4+}$. Because of the ease with which the $\mathrm{Mn}$ in these minerals can be reduced, their contribution to sustaining oxidizing-groundwater conditions should be considered (Bish and Post, 1989).

\section{SUMMARY AND CONCLUSIONS}

Fracture-lining minerals in the Paintbrush Group are highly variable in distribution, both vertically and laterally, across Yucca Mountain. The zeolites mordenite, heulandite, and stellerite are widespread in fractures in the Paintbrush Group even though this unit is generally devitrified and nonzeolitic. The silica polymorphs present in the fractures, however, generally correspond to the same species present in the matrix. The manganese-oxide minerals in this unit are lithiophorite and rancieite. Factors controlling the distribution and chemistry of fracturelining manganese oxides and zeolites in the Paintbrush Group are not yet understood, but the minerals must have formed under different geochemical conditions and possibly at different times than fracture-lining minerals in the underlying tuffs. Zeolite and calcite fracture coatings in the Paintbrush Group are important because they may provide information on water-rock interactions within the fractures and because they may be affected by increased temperatures after emplacement of waste.

Fracture-lining zeolites in the Calico Hills Formation and the Crater Flat Group include mordenite, clinoptilolite, and analcime and correlate with the zeolitic alteration of the tuffs containing them. The zeolites in fractures probably formed primarily during zeolitization of the nonwelded portions of those tuffs and are probably $\geq 10.7 \mathrm{Ma}$ in age. Mordenite is the only zeolite that occurs in devitrified intervals of these units. Manganese oxides are locally abundant in the devitrified intervals and are mostly cryptomelane-family minerals, chemically and 
structurally different from the manganese oxides in the Paintbrush Group. Although the manganese oxides occur hundreds of meters below the SWL, the $\mathrm{Mn}$ is primarily $\mathrm{Mn}^{4+}$, which can be reduced and may locally produce more oxidizing conditions in the groundwater.

\section{ACKNOWLEDGMENTS}

We wish to thank R. Raymond for electron microprobe analyses of some of the zeolites and D. Vaniman for his thoughtful review of the manuscript. The Los Alamos data-tracking numbers for this work are DAO156 (4-2-91), LA000000000020.001, LA000000000021.001, and LA-000000000096.001. The record package containing traceability information is LA-EES-1TIP-94-002. 


\section{REFERENCES}

Alietti, A. (1972). Polymorphism and crystal-chemistry of heulandites and clinoptilolites. Am. Mineral. 57, 1448-1462.

Bence, A. E., and Albee, A. L. (1968). Empirical correction factors for electron microanalysis of silicates and oxides. J. Geol. 76, 382-403.

Benson, L. V., Robison, J. H., Blankennagel, R. K., and Ogard, A. E. (1983). Chemical composition of ground water and the locations of permeable zones in the Yucca Mountain area, Nevada. U. S. Geol. Surv., Open -File Rep. 83-854, 19 pp.

Bish, D. L., and Aronson, J. L. (1993). Paleogeothermal and paleohydrologic conditions in silicic tuff from Yucca Mountain, Nevada. Clays \& Clay Minerals 41, 148-161.

Bish, D. L., and Chipera, S. J. (1991). Detection of trace amounts of erionite using X-ray powder diffraction: Erionite in tuffs of Yucca Mountain, Nevada, and central Turkey. Clays \& Clay Minerals 39, 437-445.

Bish, D. L. (1989). Evaluation of past and future alterations in tuff at Yucca Mountain, Nevada, based on the clay mineralogy of drill cores USW G-1, G-2, and G-3. Los Alamos National Laboratory report LA-10667-MS, 40 pp.

Bish, D. L., and Chipera, S. J. (1989). Revised mineralogic summary of Yucca Mountain, Nevada. Los Alamos National Laboratory report LA-11497-MS, 68 pp.

Bish, D. L., and Post, J. E. (1989). Thermal behavior of complex, tunnel-structure manganese oxides. Am. Mineral. 74, 177-186.

Boles, J. R. (1972). Composition, optical properties, cell dimensions, and thermal stability of some heulandite group zeolites. Am. Mineral. 57, 1463-1493.

Bricker, O. P. (1969). Stability constants and Gibbs free energies of formation of magadiite and kenyaite. Am. Mineral. 54, 1026-1033.

Broxton, D. E., Bish, D. L., and Warren, R. G. (1987). Distribution and chemistry of diagenetic minerals at Yucca Mountain, Nye County, Nevada. Clays \& Clay Minerals 35, 89-110.

Broxton, D. E., Warren, R. G., Hagan, R. C., and Luedemann, G. (1986). Chemistry of diagenetically altered tuffs at a potential nuclear waste repository, Yucca Mountain, Nye County, Nevada. Los Alamos National Laboratory report LA-10802-MS, 160 pp.

Buscheck, T. A., and Nitao, J. J. (1992). The impact of thermal loading on repository performance at Yucca Mountain. In Proc. 3rd Annual High-Level Radioactive Waste Management Conf., Las Vegas, Nevada, Amer. Nuclear Soc., Inc., La Grange Park, Illinois, 1003-1017.

Byers, F. M. Jr., Carr, W. J., Orkild, P. P., Quinlivan, W. D., and Sargent, K. A. (1976). Volcanic suites and related cauldrons of Timber Mountain-Oasis Valley Caldera Complex, southern Nevada. U.S. Geol. Surv. Prof. Pap. 919, 70 pp. 
Carlos, B. A., Chipera, S. J., Bish, D. L., and Raymond, R. (1995). Distribution and chemistry of fracture-lining zeolites at Yucca Mountain, Nevada. In Natural Zeolites '93, Occurrence, Properties, and Use, D. W. Ming and F. A. Mumpton, eds, International Committee on Natural Zeolites, Brockport, N. Y., 547-563.

Carlos, B. A., Chipera. S. J., Bish, D. L., and Craven, S. J. (1993). Fracture-lining manganeseoxide minerals in silicic tuff, Yucca Mountain, Nevada. Chem. Geol. 107, 47-69.

Carlos, B. A., Bish, D. L., and Chipera, S. J. (1991). Fracture-lining minerals in the Lower Topopah Spring Tuff at Yucca Mountain. In Proc. 2nd Annual High-Level Radioactive Waste Management Conf., Las Vegas, Nevada, Amer. Nuclear Soc., Inc., La Grange Park, Illinois, 486-493.

Carlos, B. A. (1989). Fracture-coating minerais in the Topopah Spring Tuff and upper tuff of Calico Hills from drill hole J-13. Los Alamos National Laboratory report LA-11504-MS, $20 \mathrm{pp}$.

Carlos, B. A. (1987). Minerals in fractures of the saturated zone from drill core USW G-4, Yucca Mountain, Nye County, Nevada. Los Alamos National Laboratory report LA10927-MS, 32 pp.

Carlos, B. A. (1985). Minerals in fractures of the unsaturated zone from drill core USW G-4, Yucca Mountain, Nye County, Nevada. Los Alamos National Laboratory report LA10415-MS, 55 pp.

Carr, W. J., Byers, F. M. Jr., and Orkild, P. P. (1986). Stratigraphic and volcano-tectonic relations of Crater Flat Group and some older volcanic units, Nye County, Nevada. U.S. Geol. Surv. Prof. Pap. 1323, 28 pp.

Chipera, S. J., Bish, D. L., and Carlos, B. A. (1995a). Equilibrium modeling of the formation of zeolites in fractures at Yucca Mountain, Nevada. In Natural Zeolites '93, Occurrence, Properties, and Use, D. W. Ming and F. A. Mumpton, eds, International Committee on Natural Zeolites, Brockport, N. Y., 565-577.

Chipera, S. J., Vaniman, D. T., Carlos, B. A., and Bish, D. L. (1995b). Mineralogic variation in drill core UE-25 UZ-16, Yucca Mountain, Nevada. Los Alamos National Laboratory report LA-12810-MS, $39 \mathrm{pp}$.

Chipera, S. J., and Bish, D. L. (1989). The occurrence and distribution of erionite at Yucca Mountain, Nevada. Los Alamos National Laboratory report LA-11663-MS, 20 pp.

Eugster, H. P. (1967). Hydrous sodium silicates from Lake Magadi, Kenya: Precursors of bedded chert. Science 157, 1177-1180.

Gottardi, G., and Galli, E. (1985). Natural Zeolites, Springer-Verlag, New York, 285-286.

Guthrie, G. D. Jr., Bish, D. L., Chipera, S. J., and Raymond, R. Jr. (1995) Distribution of potentially hazardous phases in the subsurface at Yucca Mountain, Nevada. Los Alamos National Laboratory report LA-12573-MS, 41 pp. 
Kerrisk, J. F. (1987). Groundwater chemistry at Yucca Mountain, Nevada, and vicinity. Los Alamos National Laboratory report LA-10929-MS.

Lipman, P. W., Christiansen, R. L., and O'Connor, J. T. (1966). A compositionally zoned ashflow sheet in southern Nevada. U.S. Geol. Surv. Prof. Pap. 524-F, 48 pp.

Lobmeyer, D. H., Whitfield, M. S. Jr., Lahoud, R. G., and Bruckheimer, L. (1983). Geohydrologic data for test well UE-25b\#1, Nevada Test Site, Nye County, Nevada. U.S. Geol. Surv. Open File Rept. 83-855, 48 pp.

Maldonado, F., and Koether, S. L. (1983). Stratigraphy, structure, and some petrographic features of Tertiary volcanic rocks at the USW G-2 drill hole, Yucca Mountain, Nye County, Nevada. U.S. Geol. Surv. Open-File Rept. 83-732, 83 pp.

Manceau, A., and Combes, J. M. (1988). Structure of Mn and Fe oxides and oxyhydroxides: A topological approach by EXAFS. Phys. Chem. Mineral. 15, 283-295.

Mumpton, F. A. (1960). Clinoptilolite redefined. Am. Mineral. 45, 351-369.

Post, J. E., and Bish, D. L. (1988). Rietveld refinement of the todorokite structure. Am. Mineral. 73, 861-869.

Post, J. E., Von Dreele, R. B., and Buseck, P. R. (1982). Symmetry and cation displacements in hollandites: Structure refinements of hollandite, cryptomelane and priderite. Acta Cryst. B 38,1056-1065.

Pouchou, J. L., and Pichoir, F. (1985). "PAP" $\Phi(\rho z)$ procedure for improved quantitative microanalysis. Microbeam Analysis, J. T. Armstrong, ed., San Francisco Press, San Francisco, California, 104-105.

Rimstidt, J. D., and Newcomb, W. D. (1989). A vertical thermal gradient experiment to simulate conditions in vapor dominated geothermal systems, epithermal gold deposits, and high level radioactive repositories in unsaturated media. In Proceedings of the 6th International Symposium on Water-Rock Interaction, Malvern, England, D. L. Miles, ed., Balkema, Rotterdam, 585-588.

Sawyer, D. A., Fleck, R. J., Lanphere, M. A., Warren, R. G., Broxton, D. E., and Hudson, M. R. (1994). Episodic caldera volcanism in the Miocene southwestern Nevada volcanic field: Revised stratigraphic framework, ${ }^{40} \mathrm{Ar} /{ }^{39} \mathrm{Ar}$ geochronology, and implications for magmatism and extension. GSA Bull. 106, 1304-1318.

Scott, R. B., and Castellanos, M. (1984). Stratigraphic and structural relations of volcanic rocks in drill holes USW GU-3 and USW G-3, Yucca Mountain, Nye County, Nevada. U.S. Geol. Surv. Open-File Rept. 84-491, 121 pp.

Scott, R. B., Spengler, R. W., Diehl, S., Lappin, A. R., and Chornack, M.P. (1983). Geologic character of tuffs in the unsaturated zone at Yucca Mountain, southern Nevada. In Role of the Unsaturated Zone in Radioactive and Hazardous Waste Disposal, J. W. Mercer, P. S. L. Rao, and I. W. Irvine, eds., Ann Arbor Sci Publ., Ann Arbor, Mich., 289-335. 
Spengler, R. W., and Chornack, M. P. (1984). Stratigraphic and structural characteristics of volcanic rocks in core hole USW G-4, Yucca Mountain, Nye County, Nevada. U.S. Geol. Surv. Open-File Rept. 84-789, 77 pp.

Spengler, R. W., Byers, F. M. Jr., and Warner, J. B. (1981). Stratigraphy and structure of volcanic rocks in drill hole USW G-1, Yucca Mountain, Nye County, Nevada. U.S. Geol. Surv. Open-File Rept. 81-1349, 50 pp.

Spengler, R. W., Muller, D. C., and Livermore, R. B. (1979). Preliminary report on the geology and geophysics of drill hole UE25a-1, Yucca Mountain, Nevada Test Site. U.S. Geol. Surv. Open-File Rept. 79-1244, 43 pp.

Surdam, R. C., and Eugster, H. P. (1976). Mineral reactions in the sedimentary deposits of the Lake Magadi region, Kenya. Geol. Soc. Am. Bull. 87, 1739-1752.

Thomas, K. W. (1987). Summary of sorption measurements performed with Yucca Mountain, Nevada, tuff samples and water from well J-13. Los Alamos National Laboratory report LA-10960-MS, 99 pp.

Triay, I. R., Mitchell, A. J., and Ott, M. A. (1991). Radionuclide migration as a function of mineralogy. In Proceedings of the Second Annual High-Level Radioactive Waste Management Conf., Las Vegas, Nevada, Amer. Nuclear Soc., Inc., La Grange Park, Illinois, 494-498.

WoldeGabriel, G., Broxton, D. E., Bish, D. L., and Chipera, S. J. (1993). Mineralogy and clinoptilolite KAr results from Yucca Mountain, Nevada, USA: A potential high-level radioactive waste repository site. Los Alamos National Laboratory report LA-12652-MS, $44 \mathrm{pp}$.

Yang, I. C. (1992). Flow and transport through unsaturated rock--data from two test holes, Yucca Mountain, Nevada. In Proc. 3rd Annual High-Level Radioactive Waste Management Conf., Las Vegas, Nevada, Amer. Nuclear Soc., Inc., La Grange Park, Illinois, 732-737. 


\section{APPENDIX I}

\section{QUALITATIVE MINERALOGY AS DETERMINED BY XRD}

Sample numbers are depths in feet.

\section{KEY}

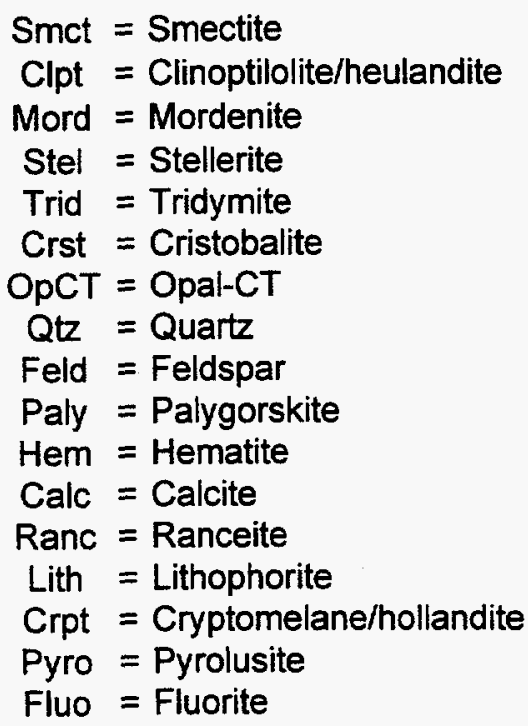

Maj $=$ Major abundance $->\approx 20 \%$ of the minerals

Min $=$ Minor-abundance $-\approx 5-20 \%$ of the minerals

Trc $=$ Trace abundance $-<\approx 5 \%$ of the minerals

$--=$ Not detected

$? \quad=$ Presence uncertain 
UE-25a\#1 and UE-25b\#1 Fracture Samples

$\begin{array}{cc}\text { Septh } \\ \text { SAMPLE } & (\mathrm{m})\end{array}$ Smet Clpt Mord Stel Trid Crst OpCT Qtz Feld Paly Hem Calc Ranc Lith Crpt Pyro Fluo OTHER

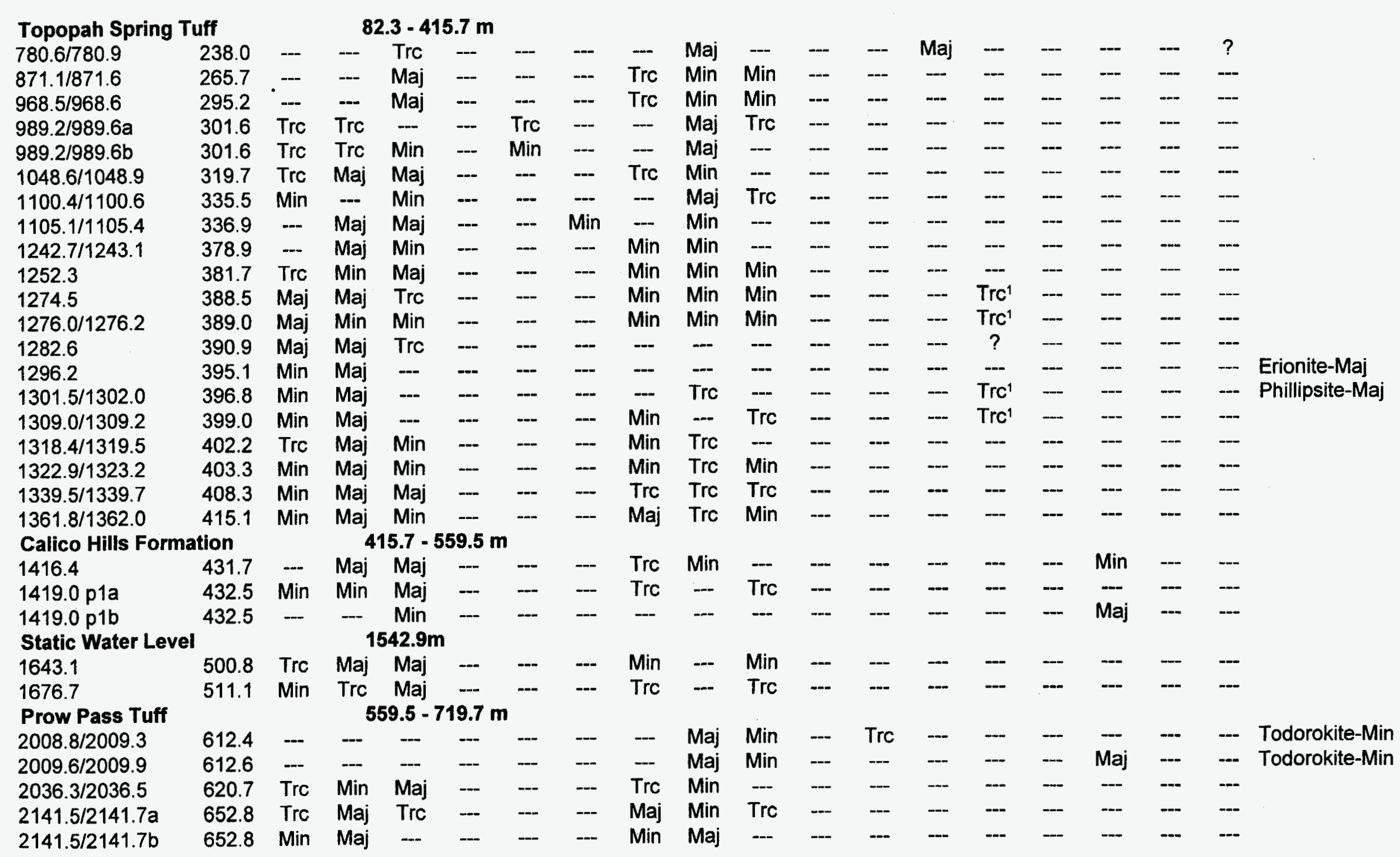


UE-25a\#1 and UE-25b1 Fracture Samples, continued

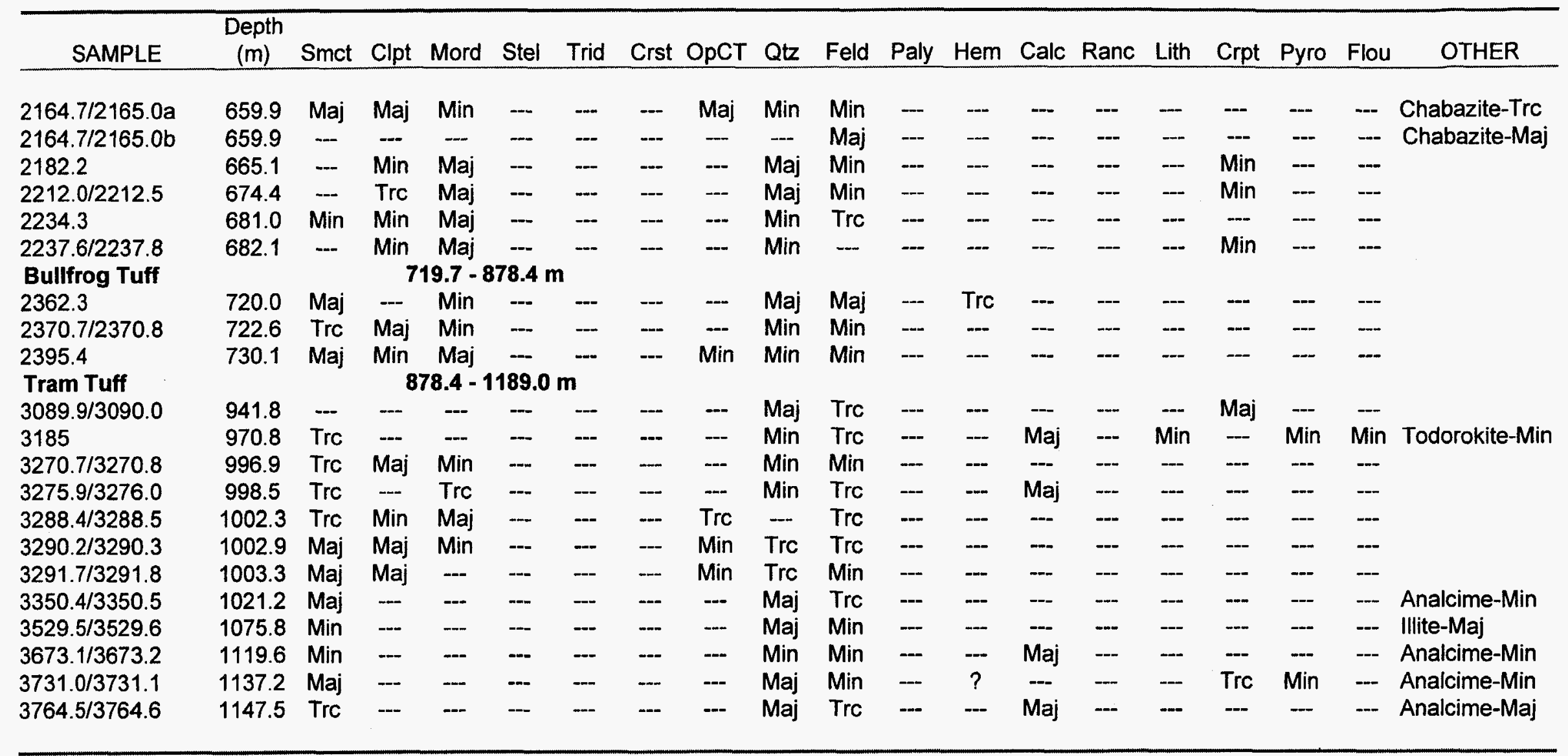

'Believed to be present based on chemistry and a single $X$-ray reflection. 
USW G-1 Fracture Samples

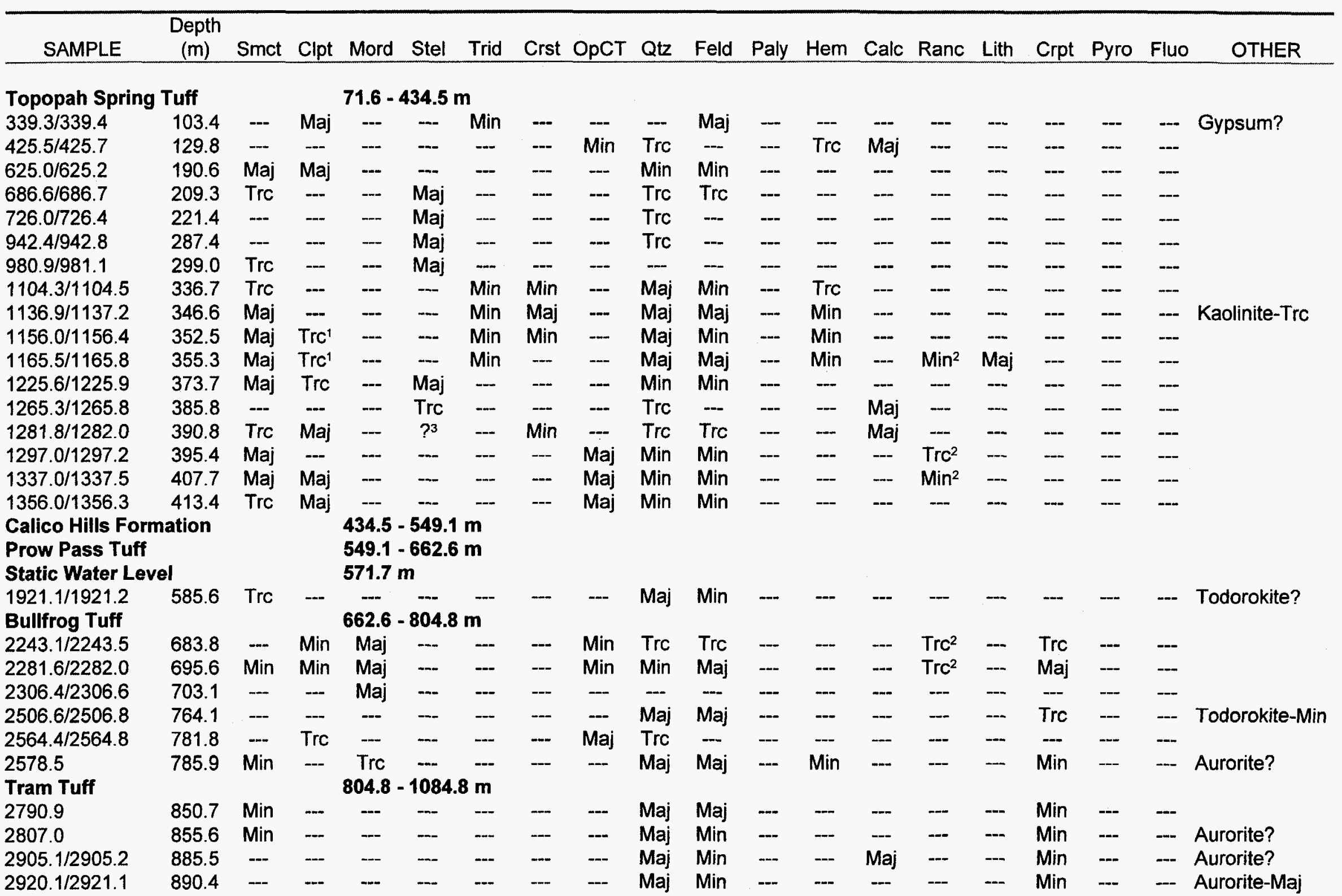


USW G-1 Fracture Samples, continued

\begin{tabular}{|c|c|c|c|c|c|c|c|c|c|c|c|c|c|c|c|c|c|c|c|}
\hline SAMPLE & $\begin{array}{c}\text { Depth } \\
\text { (m) }\end{array}$ & Smct & Clpt & Mord & Stel & Trid & Crst & OpCT & Qtz & Feld & Paly & Hem & Calc & Ranc & Lith & Crpt & Pyro & Fluo & OTHER \\
\hline $2937.2 / 2937.6 a$ & 895.4 & --- & -- & - & -- & --- & -- & --- & Trc & -- & -- & -- & Maj & -- & $\cdots$ & --- & -- & Trc & \\
\hline $2937.2 / 2937.6 b$ & 895.4 & -- & -- & -- & -- & -- & --- & -- & Trc & --- & --- & --- & Maj & -- & -- & -- & -- & Trc & \\
\hline 2963.7 & 903.3 & -- & --- & -- & -- & -- & --- & --- & Maj & Maj & -- & Trc & Tre & -- & --- & Maj & -- & - & Aurorite-Min? \\
\hline
\end{tabular}

1Zeolite--unknown whether heulandite/clinoptilolite or mordenite.

${ }^{2}$ Believed to be present based on chemistry and a single $X$-ray reflection.

sPresence uncertain because of peak overlaps with clinoptilolite. 
USW G-2 Fracture Samples

\begin{tabular}{|c|c|c|c|c|c|c|c|c|c|c|c|c|c|c|c|c|c|c|c|}
\hline SAMPLE & $\begin{array}{l}\text { Depth } \\
(\mathrm{m}) \\
\end{array}$ & Smct & Clpt & Mord & Stel & Trid & Crst & OpCT & Qtz & Feld & Paly & Hem & Calc & Ranc & Lith & Crpt & Pyro & Fluo & OTHER \\
\hline \multicolumn{3}{|c|}{ Yucca Mountain Tuff } & \multicolumn{4}{|c|}{$74.7-151.8 \mathrm{~m}$} & & & & & & & & & & & & & \\
\hline \multicolumn{2}{|c|}{$\begin{array}{l}\text { Pah Canyon Tuff } \\
\text { Topopah Spring Tuff }\end{array}$} & Maj & \multicolumn{4}{|c|}{$\begin{array}{l}151.8-231.5 \mathrm{~m} \\
231.5-535.5 \mathrm{~m}\end{array}$} & Maj & --- & -- & Min & -- & Trc & --- & Maj & -- & -- & --- & -- & Kaolinite? \\
\hline $1178.4 / 1178.7$ & 359.3 & Trc & --- & --- & --- & Min & -- & -- & Maj & Trc & $\cdots$ & Trc & -- & -- & -- & -- & --- & $-\infty$ & \\
\hline $1282.0 / 1282.1$ & 390.8 & Trc & -- & $\operatorname{Trc}^{1}$ & $-\infty$ & Maj & -- & -- & Maj & Min & --- & Min & -- & -- & -- & -- & -- & --- & \\
\hline 1447 lithoph & 441.0 & Min & -- & Min & --- & Min & Maj & --- & Min & Maj & -- & Min & --- & -- & -- & --- & --- & -- & \\
\hline 1447 breccia & 441.0 & Min & Min & Min & $?^{2}$ & Trc & Maj & -- & Maj & Maj & --- & Trc & Maj & --- & --- & -- & -- & --- & \\
\hline 1449 & 441.7 & Min & Maj & Maj & Maj & $\cdots$ & $?^{3}$ & -- & Min & Min & $\cdots$ & -- & - & -- & -- & -- & -- & -- & \\
\hline 1456 & 443.8 & Trc & Trc & Maj & --- & Trc & $?^{4}$ & -- & Min & Min & -- & -- & --- & --- & -- & $-m$ & -- & -- & \\
\hline 1488.2 & 453.6 & Min & $-\cdots$ & Trc & $?^{2}$ & $?^{3}$ & -- & --- & Maj & Min & -- & Trc & -- & --- & -- & -- & -- & -- & \\
\hline $1505.0 / 1505.2$ & 458.8 & Min & Trc & Maj & Maj & $?^{3}$ & $?^{3}$ & --- & Maj & Trc & -- & -- & $\cdots$ & --- & --- & $m$ & -- & --- & \\
\hline $1516.7 / 1517.0$ & 462.4 & Trc & -- & Maj & Maj & -- & $?^{3}$ & -- & Min & Min & --- & -- & --- & --- & -- & -- & -- & --- & \\
\hline 1534.4 & 467.7 & Trc & Trc & Maj & Trc & -- & Min & --- & Min & Min & $\cdots$ & $\cdots$ & $\cdots$ & --- & -- & - & -- & -- & \\
\hline $1568.8 / 1569.0 \mathrm{a}$ & 478.2 & Trc & Maj & Min & Maj & $-m$ & Min & -- & Trc & -- & -- & $\cdots$ & -- & --- & -- & -- & -- & --- & \\
\hline $1568.8 / 1569.0 b$ & 478.2 & Trc & Maj & Trc & Maj & -- & Min & -- & Maj & -- & --- & $\cdots$ & $-\infty$ & --- & -- & $\cdots$ & $-m$ & -- & \\
\hline $1581.0 / 1582.2$ & 482.3 & Trc & Min & Maj & Maj & -- & $?^{3}$ & --- & Min & Min & --- & --- & --- & --- & -- & -- & -- & --- & \\
\hline $1603.6 / 1604.0$ & 488.9 & Min & Maj & Maj & $?^{2}$ & -- & $?^{3}$ & --- & Min & Trc & -- & --- & -- & $\operatorname{Trc}^{6}$ & Maj & -- & --- & -- & \\
\hline $1629.7 / 1630.0$ & 496.8 & Min & Maj & $?$ & $?^{2}$ & $\cdots$ & Min & --- & Min & Min & -- & --- & -- & -- & -- & --- & -- & -- & \\
\hline $1631.2 / 1631.4$ & 497.3 & Maj & $-\cdots$ & Trc & --- & $\cdots$ & Maj & -- & Maj & Maj & -- & -- & -- & $\operatorname{Trc}^{6}$ & Min & - & -- & -- & \\
\hline $1636.7 / 1636.8$ & 498.9 & Maj & Min & -- & -- & -- & -- & --- & Trc & - & -- & --- & -- & -- & --- & --- & --- & -- & \\
\hline $1643.8 / 1644.0$ & 501.1 & Maj & Min & -- & -- & -- & -- & -- & --- & -- & $--\infty$ & --- & --- & --- & -- & $\cdots$ & --- & --- & \\
\hline $1644.0 / 1644.4 a$ & 501.2 & Min & Maj & -- & -- & -- & --- & -- & --- & -- & -- & --- & --- & --- & -- & - & -- & --- & \\
\hline $1644.0 / 1644.4 b$ & 501.2 & Maj & Maj & -- & -- & -- & -- & $-\cdots$ & --- & -- & -- & -- & -- & --- & -- & -- & --- & --- & \\
\hline 1653.4 & 504.0 & Maj & Maj & -- & $\operatorname{Trc}^{2}$ & -- & -- & Min & Trc & Trc & -- & -- & -- & -- & -- & -- & --- & -- & \\
\hline $1656.8 / 1657.2$ & 505.1 & Maj & Maj & -- & -- & -- & -- & -- & Min & --- & $-\cdots$ & --- & --- & --- & -- & $-\cdots$ & -- & -- & \\
\hline 1669 & 508.7 & Maj & Maj & Trc & -- & -- & -- & Maj & --- & Trc & --- & -- & -- & --- & --- & --- & --- & -- & \\
\hline 1680.7 & 512.3 & Min & Maj & --- & --- & $\cdots$ & - & $\mathrm{Maj}^{5}$ & Min & -- & $-\cdots$ & -- & $m$ & $\operatorname{Trc}^{6}$ & -- & -- & -- & --- & \\
\hline 1687.6 & 514.4 & Min & Maj & Trc & --- & $\cdots$ & - & Maj & Min & Trc & -- & -- & $\cdots$ & -- & -- & -- & --- & -- & \\
\hline \multicolumn{2}{|c|}{$\begin{array}{l}\text { Static Water Level } \\
\text { Calico Hills Formation }\end{array}$} & & \multicolumn{4}{|c|}{$\begin{array}{l}524.9 m \\
535.5-824.2 m\end{array}$} & & & & & & & & & & & & & \\
\hline $1757.6 / 1757.9$ & 535.8 & --- & Min & Maj & --- & -- & -- & Trc & -- & -- & -- & -- & --- & -- & -- & --- & -- & -- & \\
\hline $1762.7 / 1762.9$ & 537.3 & Min & Trc & -- & --- & -- & -- & Maj & $\cdots$ & --- & -- & -- & --- & -- & --- & --- & -- & -- & \\
\hline $1867.7 a$ & 569.3 & -- & Min & Maj & --- & -- & $\cdots$ & -- & -- & -- & --- & -- & --- & Min & -- & --- & --- & --- & \\
\hline $1867.6 b$ & 569.3 & -- & -- & Min & --- & -- & -- & --- & -- & --- & -- & --- & -- & Maj & $\cdots$ & --- & --- & -- & \\
\hline
\end{tabular}


USW G-2 Fracture Samples, continued

$\begin{array}{cc}\text { Depth } \\ \text { SAMPLE } & (\mathrm{m})\end{array}$ Smct Clpt Mord Stel Trid Crst OpCT Qtz Feld Paly Hem Calc Ranc Lith Crpt Pyro Fluo OTHER

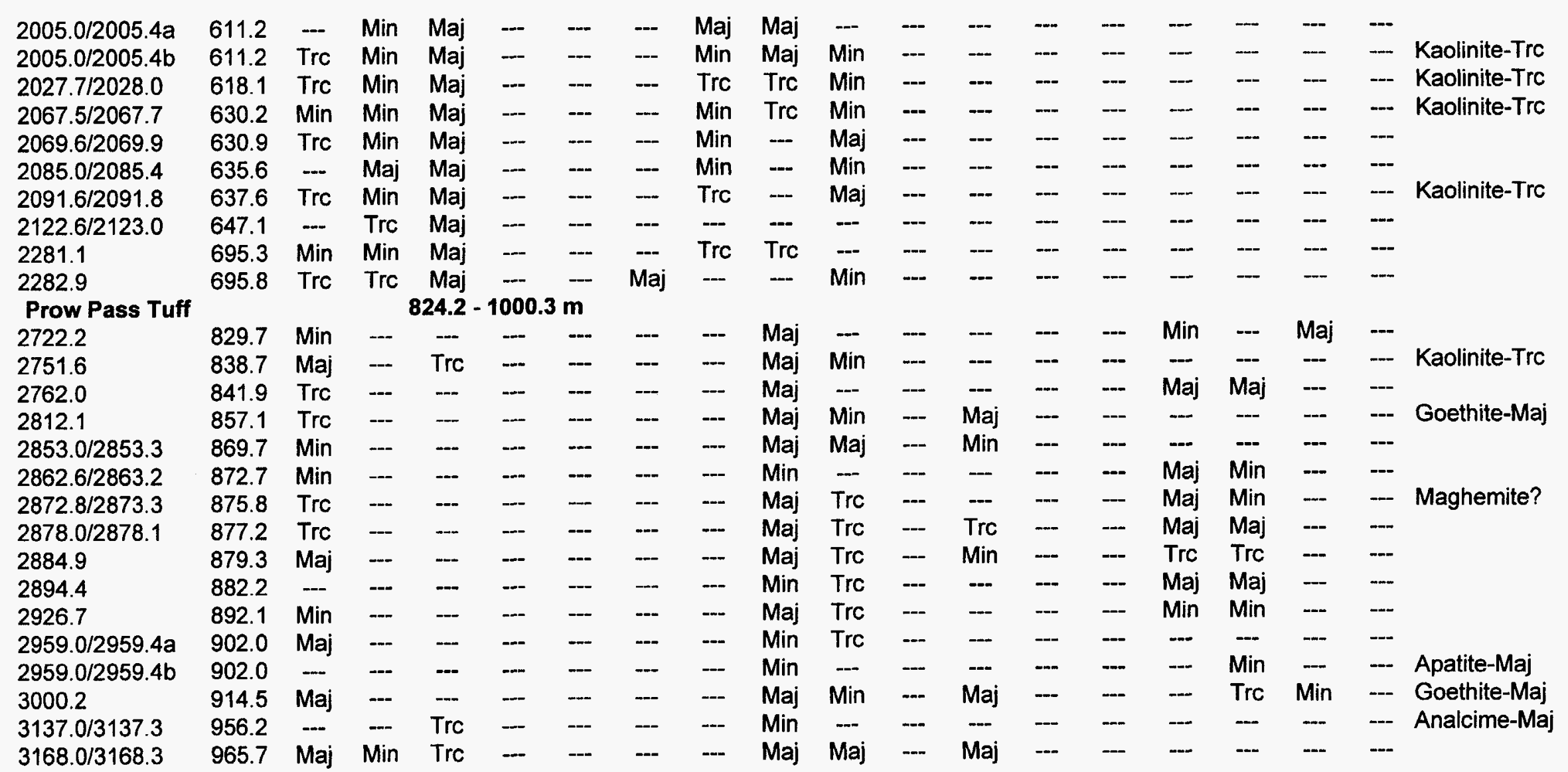


USW G-2 Fracture Samples, continued

\begin{tabular}{|c|c|c|c|c|c|c|c|c|c|c|c|c|c|c|c|c|c|c|c|}
\hline SAMPLE & $\begin{array}{c}\text { Depth } \\
\text { (m) }\end{array}$ & Smct & Clpt & Mord & Stel & Trid & Crst & OpCT & Qtz & Feld & Paly & Hem & Calc & Ranc & Lith & Crpt & Pyro & Fluo & OTHER \\
\hline Bullfrog Tuff & & & & 1000.3 & -108 & $.4 \mathrm{~m}$ & & & & & & & & & & & & & \\
\hline $3407.0 / 3407.4$ & 1038.6 & Trc & -- & -- & -- & -- & -- & --- & Maj & Maj & -- & Maj & -- & -- & -- & - & -- & -- & \\
\hline $3420.6 / 3420.9$ & 1042.7 & Trc & - & - & - & --- & $\cdots$ & $\cdots$ & Min & Min & - & -- & -- & - & Maj & -- & Maj & -- & \\
\hline
\end{tabular}

'Zeolite--unknown whether heulandite/clinoptilolite or mordenite.

2Presence uncertain because of peak overlaps with clinoptilolite and mordenite.

3Presence uncertain because of peak overlaps with stellerite.

${ }^{4}$ Presence uncertain because of peak overlaps with feldspar.

${ }^{5}$ Either poorly crystallized cristobalite or opal-C.

${ }^{6}$ Believed to be present based on chemistry and a single $X$-ray reflection. 
USW GU-3 and USW G-3 Fracture Samples

\begin{tabular}{|c|c|c|c|c|c|c|c|c|c|c|c|c|c|c|c|c|c|c|c|}
\hline SAMPLE & $\begin{array}{l}\text { Depth } \\
\text { (m) }\end{array}$ & Smct & Clpt & Mord & Stel & Trid & Crst & OpCT & $\mathrm{Qtz}$ & Feld & Paly & Hem & Calc & Ranc & Lith & Crpt & Pyro & Fluo & OTHER \\
\hline \multicolumn{3}{|c|}{ Tiva Canyon Tuff } & \multicolumn{3}{|c|}{$0-113.9 \mathrm{~m}$} & & & & & & & & & & & & & & \\
\hline 88.1 & 26.9 & --- & --- & --- & -- & Min & --- & --- & --- & -- & -- & --- & --- & -- & --- & - & --- & -- & Sepiolite-Maj \\
\hline $107.2 / 107.5$ & 32.8 & -- & --- & --- & -- & --- & --- & -- & --- & -- & $\cdots$ & -- & Maj & -- & -- & -- & -- & -- & Sepiolite-Maj \\
\hline 154.5 & 47.1 & Min & -- & --- & --- & $-\cdots$ & -- & -- & --- & -- & Maj & -- & Maj & -- & --- & -- & -- & -- & \\
\hline 244.8 & 74.6 & -- & -- & --- & -- & --- & --- & -- & --- & -- & Maj & --- & --- & -- & --- & --- & -- & -- & \\
\hline 319.0 & 97.2 & --- & Maj & $-\cdots$ & -- & -- & --- & Min & Min & -- & -- & -- & -- & --- & -- & $-\cdots$ & -- & -- & \\
\hline 337.4 & 102.8 & Maj & Min & -- & $\cdots$ & --- & -- & Min & -- & Trc & $\cdots$ & -- & -- & -- & -- & -- & -- & -- & \\
\hline 344.2 & 104.9 & Maj & -- & $\cdots$ & -- & -- & --- & Min & -- & Min & -- & --- & --- & -- & -- & -- & -- & -- & \\
\hline $354.8 / 355.3$ & 108.3 & Maj & --- & $\cdots$ & -- & -- & - & $\ldots$ & Min & Maj & --- & -- & -- & --- & -- & $\cdots$ & -- & -- & \\
\hline \multicolumn{2}{|c|}{$\begin{array}{l}\text { Paintbrush Bedded Tuffs } \\
\text { Topopah Spring Tuff }\end{array}$} & & \multicolumn{4}{|c|}{$\begin{array}{l}113.9-129.2 \mathrm{~m} \\
129.2-428.5 \mathrm{~m}\end{array}$} & & & & & & & & & & & & & \\
\hline 470.0 & 143.3 & $\cdots$ & $\cdots$ & -- & -- & -- & -- & - & - & -- & --- & -- & Maj & --- & -- & --- & -- & -- & Sepiolite-Min \\
\hline $479.7 / 480.3$ & 146.4 & -- & -- & -- & -- & -- & --- & --- & -- & -- & -- & -- & Maj & -- & -- & -- & -- & -- & Sepiolite-Maj \\
\hline $497.9 / 498.0$ & 151.8 & -- & $\cdots$ & -- & --- & Min & --- & - & -- & Maj & -- & $\ldots$ & -- & $\cdots$ & -- & --- & -- & -- & Hornblende-Min \\
\hline $517.0 / 517.1 a$ & 157.6 & Maj & --- & --- & -- & Maj & -- & -- & Trc & Min & --- & Trc & -- & -- & -- & -- & --- & -- & \\
\hline $517.0 / 517.1 \mathrm{~b}$ & 157.6 & $\cdots$ & $\cdots$ & -- & -- & Min & -- & --- & -- & Maj & -- & Tre & -- & -- & - & -- & -- & -- & \\
\hline 520.8 & 158.7 & - & - & - & -- & Tre & --- & -- & $\cdots$ & $\cdots$ & Maj & -- & --- & -- & - & -- & -- & -- & Sepiolite-Maj \\
\hline $521.6 / 521.8 a$ & 159.0 & -- & --- & --- & --. & -- & -- & $-\ldots$ & -- & -- & -- & -- & Maj & -- & - & - & - & - & \\
\hline $521.6 / 521.8 b$ & 159.0 & -- & -- & -- & -- & Min & --- & -- & -- & Min & -- & -- & Maj & -- & -- & -- & --- & -- & \\
\hline $541.0 a$ & 164.9 & -- & -- & - & - & Min & --- & -- & -- & -- & Maj & -- & -- & -- & -- & -- & -- & -- & Sepiolite-Maj \\
\hline $541.0 \mathrm{~b}$ & 164.9 & -- & -- & $\cdots$ & - & Maj & --- & -- & $-\cdots$ & Min & Min & $\cdots$ & -- & -- & -- & -- & -- & -- & Sepiolite-Min \\
\hline $579.1 / 579.5$ & 176.6 & Trc & --- & --- & --- & Maj & $\cdots$ & -- & Trc & Min & --- & Trc & -- & $\cdots$ & $\cdots$ & -. & -- & $\cdots$ & \\
\hline 610.7 & 186.1 & -- & -- & --- & --- & Maj & --- & -- & --- & Maj & -- & Tre & -- & -- & -- & --- & --- & -- & \\
\hline 687.8/688.3a & 209.8 & -- & --- & Trc & -- & --- & -- & Trc & -- & --- & -- & -- & -- & -- & -- & -- & --- & Maj & \\
\hline $687.7 / 688.3 b$ & 209.8 & -- & --- & --- & $\cdots$ & -- & $\cdots$ & - & -- & -- & -- & -- & -- & -- & -- & --- & $-\cdots$ & Maj & \\
\hline $687.7 / 688.3 c$ & 209.8 & - & - & --- & -- & $\cdots$ & Min & -- & Min & - & Maj & -- & -- & --- & $\ldots$ & --- & -- & Maj & \\
\hline 689.1 & 210.0 & Trc & -- & --- & -- & Maj & Min & --- & Min & Min & Maj & --- & Trc & -- & $\cdots$ & --- & -- & -- & \\
\hline $752.2 / 752.8$ & 229.5 & Maj & -- & $\cdots$ & -- & Min & Trc & -- & -- & -- & -- & $\cdots$ & -- & -- & Min & -- & -- & -- & \\
\hline $799.7 / 800.0$ & 243.8 & Min & --- & $\cdots$ & - & Min & $?^{1}$ & -- & Maj & Min & $\cdots$ & Min & -- & -- & -- & -- & -- & -- & \\
\hline 802.4 & 244.6 & Maj & - & Maj & -- & Min & -- & --- & Trc & -- & --- & -- & --- & -- & -- & -- & --- & $-\cdots$ & \\
\hline $804.0 / 804.3$ & 245.2 & Min & --- & -- & $\cdots$ & Maj & Min & - & Maj & Min & -- & -- & --- & $?$ & Maj & --- & --- & -- & \\
\hline $811.0 / 811.1$ & 247.2 & Maj & -- & $\operatorname{Trc}^{3}$ & $\cdots$ & Min & Min & $\cdots$ & Maj & Min & --- & Trc & $\cdots$ & -- & -- & $-\cdots$ & $\cdots$ & -- & \\
\hline $818.6 / 819.0$ & 249.6 & Maj & -- & -- & -- & Min & $?^{1}$ & -- & Maj & Min & $\cdots$ & Min & --- & $\operatorname{Trc}^{2}$ & Maj & -- & -- & -- & \\
\hline $822.0 a$ & 250.5 & Min & -- & - & --- & Maj & -- & $-\cdots$ & Min & Trc & --- & -- & -- & $\cdots$ & -- & -- & -- & -- & \\
\hline $822.0 \mathrm{~b}$ & 250.5 & Maj & -- & -- & -- & -- & Maj & -- & Min & $\min$ & -- & - & -- & $?$ & Min & $\cdots$ & -- & $-\cdots$ & \\
\hline
\end{tabular}


USW GU-3 and USW G-3 Fracture Samples, continued

\begin{tabular}{|c|c|c|c|c|c|c|c|c|c|c|c|c|c|c|c|c|c|c|c|}
\hline SAMPLE & $\begin{array}{l}\text { Depth } \\
\text { (m) }\end{array}$ & Smat & Clpt & Mord & Stel & Trid & Crst & OpCT & Qtz & Feld & Paly & $\mathrm{Hem}$ & Calc & Ranc & Lith & Crpt & Pyro & Fluo & OTHER \\
\hline $829.4 / 829.8 a$ & 252.9 & -- & -- & -- & -- & - & --- & -- & Trc & Trc & Maj & -- & Min & --- & -- & --- & - & -- & \\
\hline $829.4 / 829.8 b$ & 252.9 & Trc & -- & --- & $-\cdots$ & $\cdots$ & Min & - & Min & Trc & -- & -- & Maj & --- & -- & --- & -- & -- & \\
\hline $846.0 / 846.2$ & 257.9 & --- & -- & --- & --- & Min & --- & --- & Maj & Trc & - & Trc & -- & -- & -- & --- & --- & -- & \\
\hline $944.5 / 944.9$ & 288.0 & $\ldots$ & -- & $-\ldots$ & --- & Maj & --- & --- & Maj & Min & -- & Tre & --- & --- & Trc & -- & --- & -- & \\
\hline $945.5 / 945.7$ & 288.2 & --- & --- & --- & --- & Min & $?^{1}$ & --- & Maj & Trc & --- & -- & $?$ & -- & -- & - & -- & -- & \\
\hline $951.3 / 951.5$ & 290.0 & --- & --- & -- & --- & Maj & --- & -- & Maj & Trc & --- & -- & -- & -- & -- & -- & -- & -- & \\
\hline $972.0 / 972.2$ & 296.3 & Maj & --.- & Min & --- & $\ldots$ & Maj & --- & Min &.- & --- & --- & $\ldots$ & $\operatorname{Trc}^{2}$ & Min & -- & -- & --- & \\
\hline $973.0 / 973.1$ & 296.6 & -- & --. & --- & --- & Min & -- & --- & Maj & Trc & $\ldots$ & -- & -- & --- & --- & --- & --- & --- & \\
\hline $976.0 / 976.5$ & 297.6 & Maj & --- & - & --- & Min & Min & --- & Min & Min & --- & Trc & - & $-\ldots$ & Min & $\ldots$ & $\ldots$ & --- & \\
\hline $981.0 / 981.5$ & 299.2 & Maj & --- & $\ldots$ & -- & Maj & $?^{1}$ & - & Maj & Min & --- & -- & -- & $\mathrm{Trc}^{2}$ & Min & - & --- & -- & Chernovite?-Maj \\
\hline 987.5/988.0 & 301.1 & Min & -- & -- & --- & Maj & Min & --- & Maj & Maj & --- & Min & -- & -- & Min & -- & -- & -- & \\
\hline $1006.3 / 1006.6$ & 306.8 & -- & --- & -- & $\cdots$ & Min & $\cdots$ & -- & Maj & Trc & --- & $\cdots$ & -- & --- & -- & -- & -- & --- & \\
\hline 1072.5/1072.7\#1 & 327.0 & --- & -- & - & -- & -- & -- & -- & Min & Trc & $\cdots$ & --- & Maj & --- & - & -- & --- & Maj & \\
\hline 1072.5/1072.7\#2 & 327.0 & -- & --- & - & -- & -- & -- & -- & Min & -- & $\cdots$ & $\cdots$ & Trc & $\cdots$ & -- & -- & -- & Maj & \\
\hline $1162.7 / 1163.0$ & 354.5 & Maj & -- & Min & --- & - & Trc & $-\cdots$ & Min & Min & --- & Trc & --- & $\mathrm{Trc}^{2}$ & -- & - & -- & -- & \\
\hline $1183.0 / 1183.3$ & 360.7 & Min & Maj & -- & --- & -- & Trc & -- & Min & -- & -- & $\cdots$ & --- & -- & -- & -- & --- & -- & \\
\hline 1189.3/1189.6á & 362.6 & Min & Maj & -- & --- & -- & --- & Min & Maj & Trc & -- & -- & $-\cdots$ & $\operatorname{Trc}^{2}$ & --- & -- & -- & -- & Erionite-Maj, Other \\
\hline $1189.3 / 1189.6 b$ & 362.6 & Min & Maj & -- & -- & -- & $\cdots$ & Trc & Maj & -- & -- & -- & -- & -- & -- & -- & -- & --- & Erionite-Trc, Other ${ }^{4}$ \\
\hline 1189.3/1189.6c & 362.6 & -- & Maj & -- & --- & -- & -- & Min & Maj & Tre & -.. & --- & --- & -- & -- & --- & --- & --- & \\
\hline $1200.1 / 1200.6 \# 1$ & 365.9 & Min & Min & -- & --- & --- & -- & -- & -- & -- & --- & --- & --- & --- & --- & --- & --- & -- & Phillipsite-Maj \\
\hline $1200.1 / 1200.6 \# 2$ & 365.9 & Min & Min & - & -- & -- & -- & -- & Min & -- & --- & --- & --- & --- & -- & -- & -- & -- & Phillipsite-Maj \\
\hline $1210.2 / 1210.6 a$ & 369.0 & Maj & -- & $\mathrm{Trc}^{3}$ & --- & $\cdots$ & Min & -- & Min & Min & - & --- & --- & $\mathrm{Trc}^{2}$ & --- & --- & --- & Maj & \\
\hline $1210.2 / 1210.6 b$ & 369.0 & Min & -- & -- & -- & --- & - & --- & $\cdots$ & -- & --- & -- & -- & -- & -- & -- & $\cdots$ & Maj & \\
\hline 1232.0/1232.1 & 375.5 & Maj & $\cdots$ & -- & -- & --- & Min & --- & -- & Trc & -- & -- & -- & $\mathrm{Trc}^{2}$ & --- & --- & --- & Maj & \\
\hline \multicolumn{20}{|c|}{$\begin{array}{l}\text { Bedded Tuffs - Calico Hills Formation } 428.5-475.6 \mathrm{~m} \\
\text { Prow Pass Tuff } \\
475.6-607.1 \mathrm{~m}\end{array}$} \\
\hline $1713.7 / 1714.2$ & 522.5 & Trc & --- & -- & --- & -- & -- & -- & Maj & Min & --- & Trc & Maj & -- & -- & -- & --- & -- & \\
\hline 1714.2 & 522.5 & Trc & -- & Maj & -- & - & Min & --- & Min & Min & --- & -- & Min & --- & -- & --- & --- & Maj & \\
\hline $1734.3 / 1734.6$ & 528.7 & Min & --- & Trc & --- & --- & Maj & -- & Min & Maj & --- & Trc & $?$ & -- & -- & -- & --- & --- & \\
\hline 1936.8/1937.1 & 590.4 & -- & Maj & - & - & --- & -- & Min & -- & Trc & -- & -- & -- & -- & --- & -- & -- & --- & \\
\hline Bullfrog Tuff & & & & $11.0-$ & 97.7 & & & & & & & & & & & & & & \\
\hline 2013.4/2013.7a & 613.8 & Maj & Min & -- & --- & --- & - & -- & Min & Min & -- & - & -- & -- & - & -- & --- & --- & Other ${ }^{5}-$ Min \\
\hline 2013.4/2013.7b & 613.8 & Min & Maj & -- & --- & --- & - & Min & - & Min & -- & --- & --- & -- & - & --- & -- & --- & \\
\hline 2094.7 & 638.5 & Trc & --- & - & --- & -- & -- & --- & Maj & Min & --- & Trc & - & -- & -- & -- & -- & --- & \\
\hline $2188.5 / 2188.9$ & 656.7 & Trc & --- & Trc & --- & -- & --- & $-\ldots$ & Maj & Min & -- & -- & -- & -- & -- & -- & -- & --- & \\
\hline
\end{tabular}


USW GU-3 and USW G-3 Fracture Samples, continued

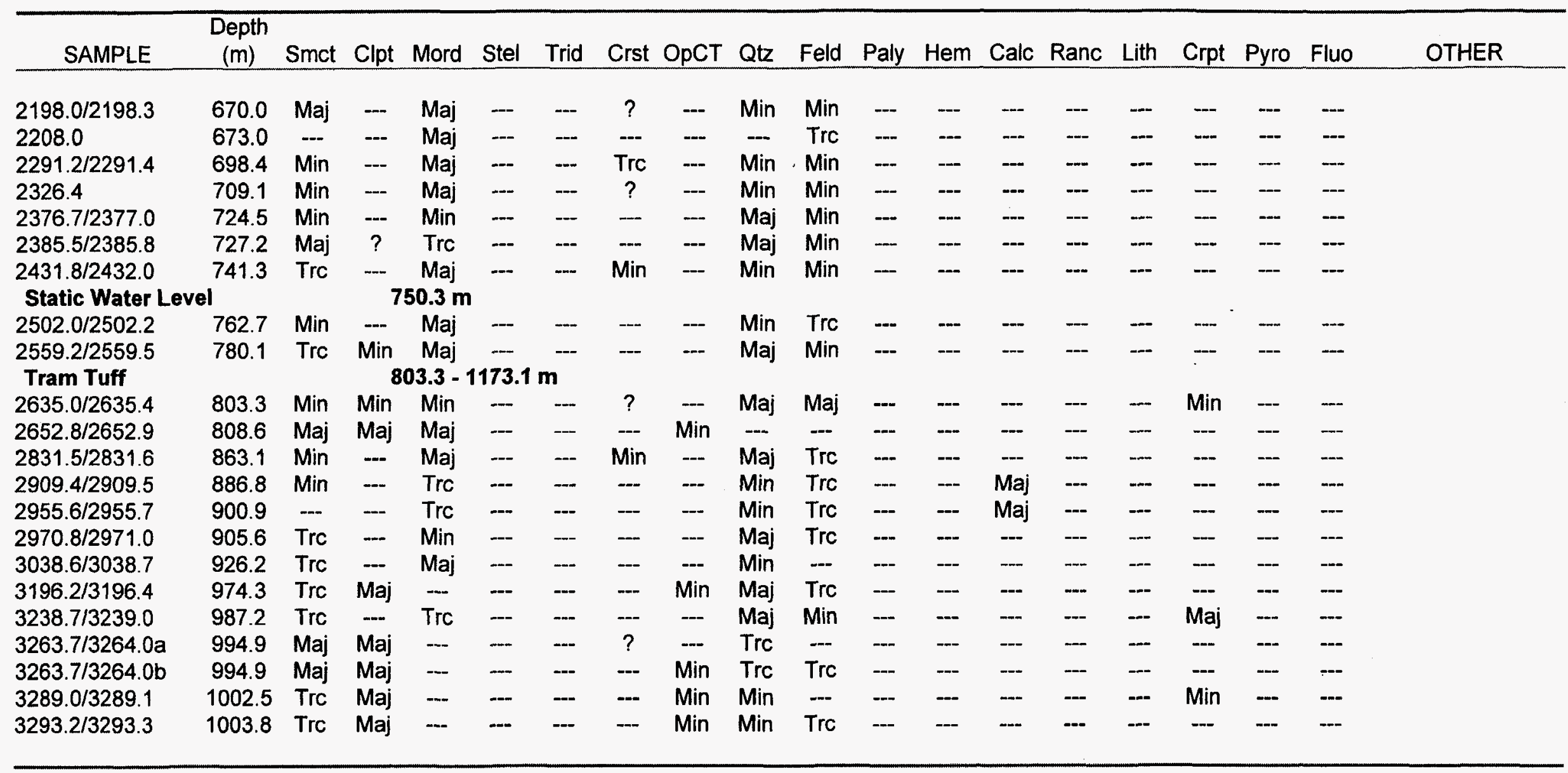

'Believed to be present but positive identification impossible because of feldspar or tridymite peak overlaps.

${ }^{2}$ Believed to be present based on chemistry and a single $X$-ray reflection.

${ }^{3}$ Zeolite--unknown whether heulandite/clinoptilolite or mordenite.

${ }^{4}$ Major abundance of an unknown phase believed to be kenyaite.

${ }^{5}$ Minor abundance of an unidentified phase. 


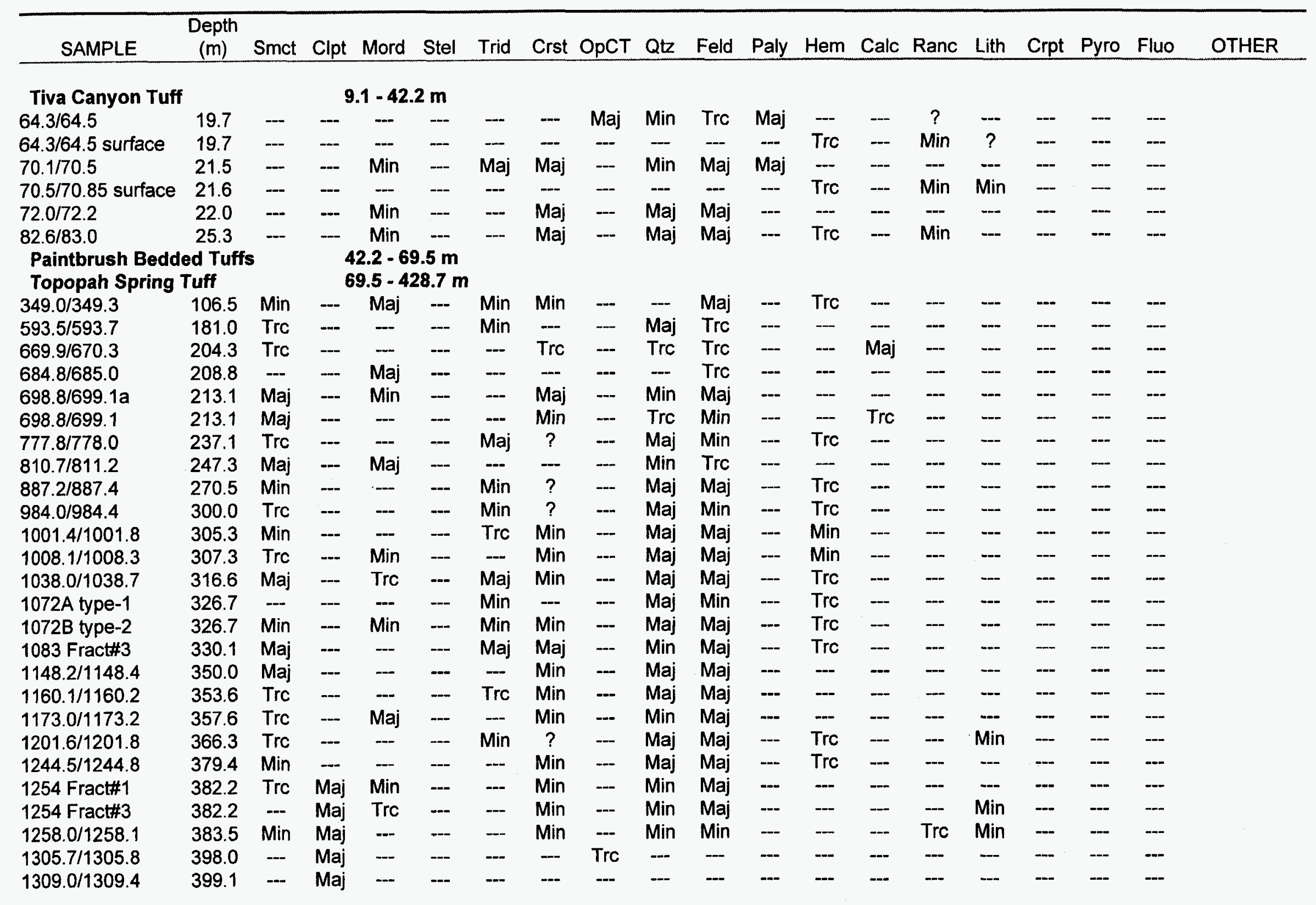


USW G-4 Fracture Samples, continued

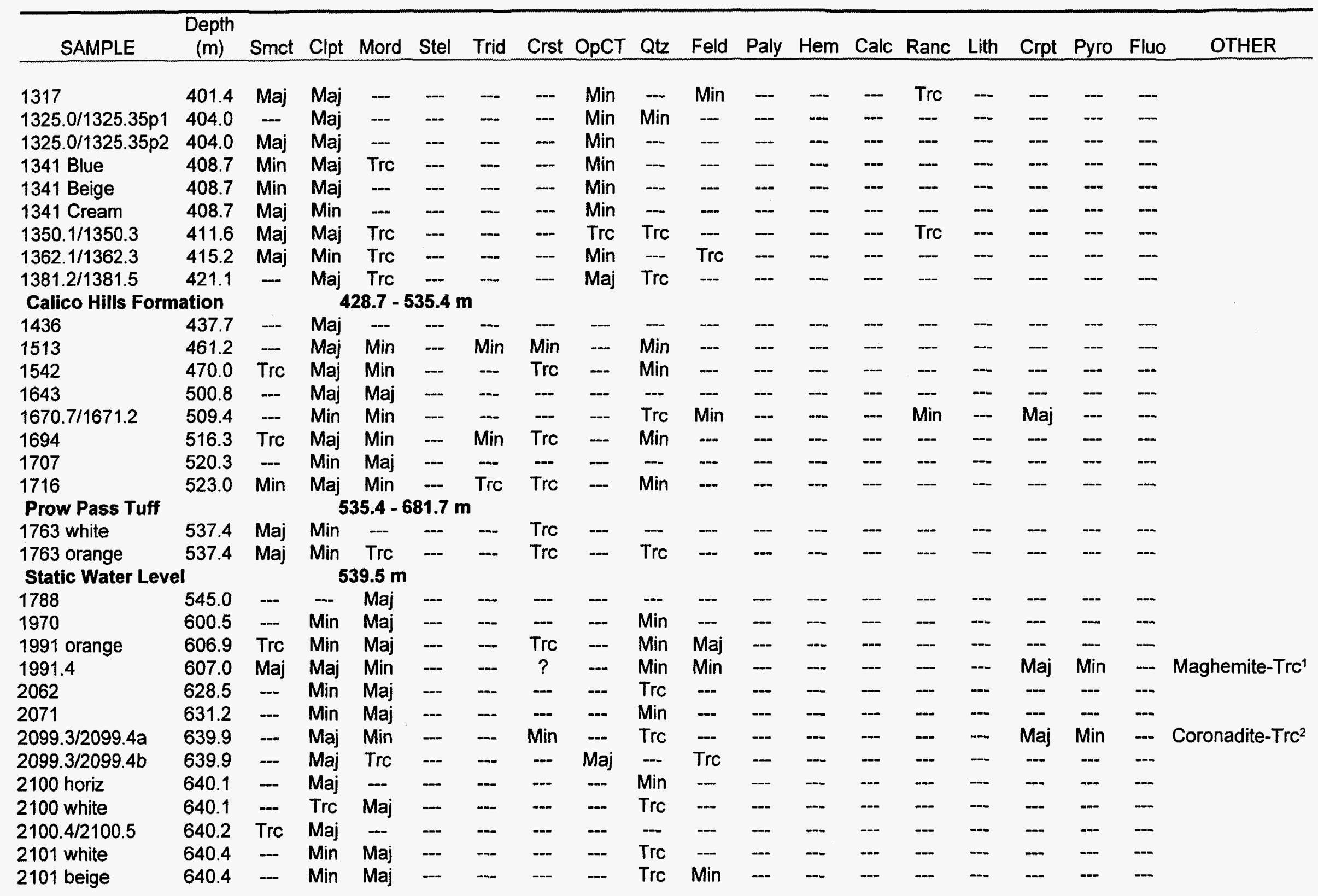


USW G-4 Fracture Samples, continued

\begin{tabular}{|c|c|c|c|c|c|c|c|c|c|c|c|c|c|c|c|c|c|c|c|}
\hline SAMPLE & $\begin{array}{c}\text { Depth } \\
(\mathrm{m})\end{array}$ & Smct & Clpt & Mord & Stel & Trid & Crst & OpCT & Qtz & Feld & Paly & Hem & Calc & Ranc & Lith & Crpt & Pyro & Fluo & OTHER \\
\hline 2135 & 650.7 & --- & --- & - & -- & -- & Maj & --- & -- & $\cdots$ & $\cdots$ & --- & --- & - & --- & -- & -- & -- & \\
\hline 2147 white & 654.4 & --- & Maj & Maj & -- & --- & -- & -- & Trc & -- & -- & -- & --- & -- & -- & -..- & -- & -- & \\
\hline 2147 pink & 654.4 & . - .- & Min & Maj & --- & -- & -- & --- & Trc & Trc & -- & -- & -- & -- & $\cdots$ & --- & --- & -- & \\
\hline Bullfrog Tuff & & & & $31.7-8$ & 36.1 & & & & & & & & & & & & & & \\
\hline 2248 & 685.2 & --- & Maj & Maj & $-\cdots$ & -- & -- & -- & Trc & Min & --- & --- & -- & -- & --- & --- & -- & -- & \\
\hline 2344 & 714.5 & -- & $\cdots$ & -- & --- & - & Trc & -- & Maj & Maj & --- & --- & -- & -- & --- & - & -- & -- & \\
\hline 2578 & 785.8 & --- & -- & --- & -- & --- & Trc & -- & Maj & Min & -- & --- & Maj & - & --- & --- & -- & --- & \\
\hline $2615.8 / 2615.9$ & 797.3 & Trc & -- & --- & --- & --- & - & --- & Maj & Maj & -- & -- & -- & -- & -- & Maj & --- & --- & Todorokite-Min \\
\hline 2689 & 819.6 & Maj & -- & --- & -- & - & -- & -- & Maj & Min & -- & -- & --- & -- & -- & --- & --- & -- & \\
\hline 2698 & 822.4 & -- & Maj & Maj & --- & -- & Min & -- & -- & -- & -- & -- & -- & $\cdots$ & -- & --- & -- & $-\cdots$ & \\
\hline 2728 & 831.5 & -- & Trc & Maj & $\cdots$ & -- & -- & -- & Trc & -- & -- & -- & -- & -- & -- & --- & --- & -- & \\
\hline Tram Tuff & & & & $36.1 \mathrm{~m}$ & - bot & & & & & & & & & & & & & & \\
\hline 2793 & 851.3 & --- & Maj & Min & $-\cdots$ & -- & -- & --- & -- & $\cdots$ & -- & -- & -- & $\cdots$ & -- & -- & --- & $\cdots$ & \\
\hline 2823 & 860.5 & Trc & Trc & Maj & -- & -- & -- & -- & Min & --- & -- & -- & -- & -- & -- & -- & -- & -- & \\
\hline 2832 & 863.2 & Min & Min & Maj & --- & -- & -- & -- & Min & -- & -- & --- & -- & --- & -- & --- & -- & -- & \\
\hline $2854.5 / 2854.7$ & 870.1 & Trc & -- & $-\infty$ & --- & -- & -- & --- & Maj & Maj & --- & Min & --- & $\cdots$ & --- & Min & $?$ & --- & \\
\hline $2886.2 / 2886.5$ & 879.8 & Trc & --- & $\ldots$ & -- & -- & -- & -- & Maj & Maj & --- & Min & -- & -- & --- & -- & -- & --- & Rhodocrosite? \\
\hline $2904.8 / 2904.9$ & 885.4 & -- & -- & --- & - & --- & -- & $\cdots$ & Maj & Maj & -- & Tre & -- & - & --- & - & -- & -- & \\
\hline 2917 & 889.1 & Trc & --- & --- & --- & -- & --- & -- & Maj & Maj & -- & Trc & -- & - & -- & Maj & $?$ & --- & Todorokite? \\
\hline $2917.2 / 2917.3$ & 889.2 & -- & -- & --- & -- & -- & -- & --- & Maj & Min & -- & -- & Trc & -- & --- & Maj & -- & $\cdots$ & \\
\hline $2920.5 / 2920.6$ & 890.2 & Min & -- & -- & $\cdots$ & --- & -- & -- & Maj & Maj & -- & Min & -- & -- & --- & -- & - & - & \\
\hline $2923.3 / 2923.5$ & 891.1 & -- & -- & -- & --- & -- & -- & --- & Maj & Min & -- & -- & --- & -- & - & Maj & -- & -- & \\
\hline 2931 & 893.4 & Maj & -- & Min & --- & -- & - & - & Maj & --- & -- & -- & -- & -- & --- & -- & -- & --- & \\
\hline 2947.3 & 898.3 & Trc & -- & -- & -- & -- & -- & --- & Maj & Min & -- & -- & -- & --- & --- & Maj & $?$ & -- & Todorokite? \\
\hline $2952.7 / 2953.2$ & 900.1 & -- & --- & -- & --- & --- & - & $\cdots$ & Min & Min & -- & $\cdots$ & -- & --- & --- & Maj & - & --- & Todorokite-Trc \\
\hline $2954.8 / 2955.1$ & 900.7 & Min & --- & -- & -- & -- & -- & -- & Maj & Min & -- & --- & -- & -- & --- & Maj & $?$ & --- & Todorokite-Min \\
\hline $2967.4 / 2967.5$ & 904.5 & Trc & -- & -- & -- & -- & -- & -- & Maj & Min & -- & -- & -- & -- & --- & Maj & $?$ & --- & Todorokite? \\
\hline $2967.5 / 2967.8 a$ & 904.6 & Maj & -- & Min & -- & -- & - & - & Min & -- & -- & --- & --- & -- & --- & -- & -- & --- & \\
\hline $2967.5 / 2967.8 b$ & 904.6 & -- & --- & --- & --- & -- & - & --- & Maj & Min & -- & --- & Trc & -- & --- & Maj & $?$ & --- & \\
\hline $2980.8 / 2981.3$ & 908.7 & -- & -- & --- & --- & -- & - & -- & Maj & Min & - & $\cdots$ & --- & --- & $\cdots$ & Min & $\cdots$ & --- & Todorokite-Trc \\
\hline $2985.6 / 2986.0$ & 910.1 & Min & -- & --- & -- & -- & -- & -- & Maj & Min & -- & Trc & --- & --- & -- & Min & $\cdots$ & -- & \\
\hline $2986.5 / 2986.8 a$ & 910.4 & -- & --- & -- & --- & --- & -- & $-\cdots$ & Maj & Maj & -- & Min & -- & -- & $\cdots$ & --- & --- & --- & \\
\hline $2986.5 / 2986.8 b$ & 910.4 & -- & --- & - & --- & --- & -- & -- & Maj & Min & -- & -- & -- & -- & -- & Maj & $\cdots$ & -- & \\
\hline
\end{tabular}

'Magnetite or maghemite; oxidation state is unknown.

${ }^{2}$ Coronadite; identification made using an optical microscope and electron microprobe. 


\section{APPENDIX $\|$}

FRACTURE-LINING ZEOLITES IN THE TOPOPAH SPRING TUFF

Data were collected on Cameca MBX and SX-50 electron microprobes.

Analyses were accepted if totals were $>84 \%$ and $\mathrm{Al} / \Sigma$ cations was between 0.95 and 1.05 .

Data acquired before 1993 were not included in the tables because Sr was not included in the analyses.

High background counts for $\mathrm{Ba}$ on the SX-50 resulted in a $\mathrm{BaO}$ detection limit of $0.33 \mathrm{wt} \%$.

Sample numbers indicate drill hole and depth in feet.

$\mathrm{n} / \mathrm{a}=$ not analyzed

- = not detected 
UE-25a\#1 1242

\begin{tabular}{|c|c|c|c|c|c|c|c|c|c|c|c|c|c|c|c|}
\hline $\begin{array}{l}\mathrm{SiO}_{2} \\
\mathrm{Al}_{2} \mathrm{O}_{3} \\
\mathrm{Fe}_{2} \mathrm{O}_{3}\end{array}$ & $\begin{array}{r}62.81 \\
14.36 \\
-\end{array}$ & $\begin{array}{r}64.63 \\
14.85 \\
-\end{array}$ & $\begin{array}{r}65.85 \\
15.12 \\
-\end{array}$ & $\begin{array}{l}63.87 \\
15.02\end{array}$ & $\begin{array}{l}64.84 \\
14.40\end{array}$ & $\begin{array}{l}65.68 \\
14.73\end{array}$ & $\begin{array}{r}66.19 \\
15.25 \\
0.05\end{array}$ & $\begin{array}{l}64.19 \\
14.82\end{array}$ & $\begin{array}{l}65.71 \\
14.56\end{array}$ & $\begin{array}{l}64.48 \\
15.06\end{array}$ & $\begin{array}{l}65.48 \\
14.92\end{array}$ & $\begin{array}{l}64.56 \\
15.09\end{array}$ & $\begin{array}{r}65.31 \\
14.90 \\
0.05\end{array}$ & $\begin{array}{l}62.13 \\
14.53\end{array}$ & $\begin{array}{l}63.72 \\
14.10\end{array}$ \\
\hline $\mathrm{MgO}$ & 1.83 & 1.80 & 1.80 & 1.90 & 1.80 & 1.87 & 1.86 & 1.88 & 1.86 & 1.98 & 1.88 & 1.78 & 1.76 & 1.85 & 1.80 \\
\hline $\mathrm{CaO}$ & 4.61 & 4.60 & 4.54 & 4.73 & 4.39 & 4.58 & 4.62 & 4.63 & 4.52 & 4.62 & 4.50 & 4.81 & 4.76 & 4.53 & 4.45 \\
\hline SrO & 0.75 & 0.88 & 0.78 & 0.78 & 0.68 & 0.75 & 0.80 & 0.80 & 0.66 & 0.77 & 0.70 & 0.99 & 0.89 & 0.85 & 0.72 \\
\hline $\mathrm{BaO}$ & & & - & - & - & - & - & - & - & - & - & - & - & - & \\
\hline $\mathrm{Na}_{2} \mathrm{O}$ & 0.53 & 0.53 & 0.45 & 0.41 & 0.63 & 0.53 & 0.50 & 0.47 & 0.57 & 0.41 & 0.57 & 0.48 & 0.42 & 0.27 & 0.40 \\
\hline $\mathrm{K}_{2} \mathrm{O}$ & 0.17 & 0.25 & 0.20 & 0.18 & 0.20 & 0.22 & 0.20 & 0.23 & 0.22 & 0.20 & 0.26 & 0.22 & 0.26 & 0.11 & 0.19 \\
\hline Total & 85.06 & 87.54 & 88.74 & 86.89 & 86.94 & 88.36 & 89.47 & 87.02 & 88.10 & 87.52 & 88.31 & 87.93 & 88.35 & 84.27 & 85.38 \\
\hline $\mathrm{Si}$ & 28.31 & 28.32 & 28.39 & 28.19 & 28.53 & 28.46 & 28.33 & 28.28 & 28.53 & 28.23 & 28.39 & 28.21 & 28.36 & 28.24 & 28.54 \\
\hline $\mathrm{Al}$ & 7.63 & 7.67 & 7.68 & 7.81 & 7.47 & 7.52 & 7.69 & 7.70 & 7.45 & 7.77 & 7.62 & 7.77 & 7.62 & 7.78 & 7.44 \\
\hline $\mathrm{Fe}$ & - & - & & & & & 0.02 & - & - & & & & 0.02 & - & \\
\hline Mg & 1.23 & 1.18 & 1.16 & 1.25 & 1.18 & 1.21 & 1.19 & 1.23 & 1.20 & 1.29 & 1.21 & 1.16 & 1.14 & 1.25 & 1.20 \\
\hline $\mathrm{Ca}$ & 2.23 & 2.16 & 2.10 & 2.24 & 2.07 & 2.13 & 2.12 & 2.19 & 2.10 & 2.17 & 2.09 & 2.25 & 2.21 & 2.21 & 2.14 \\
\hline $\mathrm{Sr}$ & 0.20 & 0.22 & 0.20 & 0.20 & 0.17 & 0.19 & 0.20 & 0.20 & 0.17 & 0.20 & 0.18 & 0.25 & 0.22 & 0.22 & 0.19 \\
\hline $\mathrm{Na}$ & 0.46 & 0.45 & 0.38 & 0.35 & 0.54 & 0.45 & 0.41 & 0.40 & 0.48 & 0.35 & 0.48 & 0.41 & 0.35 & 0.24 & 0.35 \\
\hline K & 0.10 & 0.14 & 0.11 & 0.10 & 0.11 & 0.12 & 0.11 & 0.13 & 0.12 & 0.11 & 0.14 & 0.12 & 0.14 & 0.06 & 0.11 \\
\hline Total & 40.15 & 40.14 & 40.01 & 40.13 & 40.07 & 40.07 & 40.07 & 40.13 & 40.05 & 40.11 & 40.11 & 40.17 & 40.07 & 40.01 & 39.97 \\
\hline $\mathrm{O}^{-2}$ & 72.00 & 72.00 & 72.00 & 72.00 & 72.00 & 72.00 & 72.00 & 72.00 & 72.00 & 72.00 & 72.00 & 72.00 & 72.00 & 72.00 & 72.00 \\
\hline ¿cations & 7.87 & 7.71 & 7.38 & 7.82 & 7.50 & 7.61 & 7.53 & 7.78 & 7.55 & 7.77 & 7.59 & 7.85 & 7.65 & 7.67 & 7.50 \\
\hline $\mathrm{Al} / \Sigma \mathrm{ca}$ & 0.97 & 0. & 1.0 & 1.00 & 1.00 & 0.99 & 1.02 & 0.99 & 0.99 & 1.00 & 1.01 & 0.9 & 1.00 & 1.01 & 0.99 \\
\hline Si/Al & 3.71 & 3.69 & 3.70 & 3.61 & 3.82 & 3.78 & 3.68 & 3.68 & 3.83 & 3.63 & 3.72 & 3.6 & 3.72 & 3.63 & 3.83 \\
\hline $\mathrm{Ca}+\mathrm{Mg}+\mathrm{Sr}$ & 3.65 & 3.56 & 3.45 & 3.69 & 3.42 & 3.52 & 3.50 & 3.62 & 3.47 & 3.65 & 3.48 & 3.66 & 3.58 & 3.68 & 3.52 \\
\hline
\end{tabular}


Prismatic (small) Heulandite in the Devitrified Topopah Spring Tuff, continued

\begin{tabular}{|c|c|c|c|c|c|c|c|c|c|c|c|c|c|}
\hline \multirow[b]{2}{*}{$\mathrm{SiO}_{2}$} & \multicolumn{3}{|c|}{ UE-25a\#1 1242} & \multicolumn{4}{|c|}{ USW G-2 1488} & \multicolumn{6}{|c|}{ USW G-2 1505} \\
\hline & 62.39 & 62.65 & 62.10 & 64.78 & 64.81 & 64.37 & 64.77 & 64.53 & 64.94 & 64.15 & 64.97 & 63.94 & 64.53 \\
\hline $\mathrm{Al}_{2} \mathrm{O}_{3}$ & 14.14 & 14.38 & 14.68 & 14.95 & 14.94 & 15.07 & 15.09 & 15.27 & 15.37 & 15.10 & 15.14 & 14.94 & 14.91 \\
\hline $\mathrm{Fe}_{2} \mathrm{O}_{3}$ & & & & - & - & - & - & - & - & - & - & & \\
\hline $\mathrm{MgO}$ & 1.71 & 1.73 & 1.76 & 1.46 & 1.60 & 1.47 & 1.43 & 1.59 & 1.40 & 1.42 & 1.21 & 0.70 & 0.83 \\
\hline $\mathrm{CaO}$ & 4.30 & 4.39 & 4.67 & 4.75 & 4.85 & 5.09 & 5.10 & 5.00 & 5.14 & 5.26 & 5.28 & 5.39 & 5.28 \\
\hline SrO & 0.78 & 0.82 & 0.94 & 0.70 & 0.67 & 0.83 & 0.76 & 0.77 & 0.89 & 0.73 & 0.87 & 1.12 & 1.01 \\
\hline $\mathrm{BaO}$ & & & - & - & - & - & - & - & - & - & - & - & - \\
\hline $\mathrm{Na}_{2} \mathrm{O}$ & 0.52 & 0.31 & 0.36 & 0.37 & 0.34 & 0.33 & 0.28 & 0.22 & 0.34 & 0.29 & 0.35 & 0.39 & 0.36 \\
\hline $\mathrm{K}_{2} \mathrm{O}$ & 0.22 & 0.17 & 0.22 & 0.85 & 0.67 & 0.76 & 0.79 & 0.59 & 0.83 & 0.47 & 0.85 & 1.19 & 1.09 \\
\hline Total & 84.06 & 84.45 & 84.73 & 87.86 & 87.88 & 87.92 & 88.22 & 87.97 & 88.91 & 87.42 & 88.67 & 87.67 & 88.01 \\
\hline $\mathrm{Si}$ & 28.43 & 28.39 & 28.15 & 28.34 & 28.32 & 28.21 & 28.26 & 28.19 & 28.16 & 28.21 & 28.26 & 28.26 & 28.34 \\
\hline $\mathrm{Al}$ & 7.59 & 7.68 & 7.84 & 7.71 & 7.69 & 7.78 & 7.76 & 7.86 & 7.86 & 7.82 & 7.76 & 7.78 & 7.72 \\
\hline $\mathrm{Fe}$ & & - & - & - & - & - & - & - & - & - & - & - & - \\
\hline $\mathrm{Mg}$ & 1.16 & 1.17 & 1.19 & 0.95 & 1.04 & 0.96 & 0.93 & 1.04 & 0.91 & 0.93 & 0.78 & 0.46 & 0.54 \\
\hline $\mathrm{Ca}$ & 2.10 & 2.13 & 2.27 & 2.23 & 2.27 & 2.39 & 2.38 & 2.34 & 2.39 & 2.48 & 2.46 & 2.55 & 2.48 \\
\hline $\mathrm{Sr}$ & 0.21 & 0.22 & 0.25 & 0.18 & 0.17 & 0.21 & 0.19 & 0.20 & 0.22 & 0.19 & 0.22 & 0.29 & 0.26 \\
\hline $\mathrm{Na}$ & 0.46 & 0.27 & 0.32 & 0.31 & 0.29 & 0.28 & 0.24 & 0.19 & 0.29 & 0.25 & 0.30 & 0.33 & 0.31 \\
\hline $\mathrm{K}$ & 0.13 & 0.10 & 0.13 & 0.47 & 0.37 & 0.42 & 0.44 & 0.33 & 0.46 & 0.26 & 0.47 & 0.67 & 0.61 \\
\hline Total & 40.07 & 39.96 & 40.15 & 40.20 & 40.16 & 40.25 & 40.20 & 40.14 & 40.28 & 40.14 & 40.25 & 40.35 & 40.26 \\
\hline $\mathrm{O}^{-2}$ & 72.00 & 72.00 & 72.00 & 72.00 & 72.00 & 72.00 & 72.00 & 72.00 & 72.00 & 72.00 & 72.00 & 72.00 & 72.00 \\
\hline Lcations & 7.52 & 7.40 & 7.85 & 7.50 & 7.63 & 7.83 & 7.69 & 7.66 & 7.78 & 7.70 & 7.70 & 7.61 & 7.49 \\
\hline Al/Ications & 1.01 & 1.04 & 1.00 & 1.03 & 1.01 & 0.99 & 1.01 & 1.03 & 1.01 & 1.02 & 1.01 & 1.02 & 1.03 \\
\hline $\mathrm{Si} / \mathrm{Al}$ & 3.74 & 3.70 & 3.59 & 3.68 & 3.68 & 3.62 & 3.64 & 3.59 & 3.58 & 3.60 & 3.64 & 3.63 & 3.67 \\
\hline $\mathrm{Ca}+\mathrm{Mg}+\mathrm{Sr}$ & 3.47 & 3.52 & 3.71 & 3.36 & 3.48 & 3.56 & 3.51 & 3.57 & 3.52 & 3.59 & 3.46 & 3.30 & 3.29 \\
\hline
\end{tabular}


Tabular (large) Heulandite in the Devitrified Topopah Spring Tuff

USW G-4 1258

$\begin{array}{lrrrrrrr}\mathrm{SiO}_{2} & 62.07 & 62.17 & 62.70 & 62.18 & 62.29 & 62.42 & 61.40 \\ \mathrm{Al}_{2} \mathrm{O}_{3} & 14.45 & 14.37 & 14.28 & 14.46 & 14.52 & 14.52 & 14.89 \\ \mathrm{Fe}_{2} \mathrm{O}_{3} & - & - & - & - & - & - & - \\ \mathrm{MgO} & 0.29 & 0.30 & - & 0.14 & 0.39 & - & 0.31 \\ \mathrm{CaO} & 5.18 & 5.32 & 5.18 & 5.27 & 5.12 & 5.20 & 5.37 \\ \mathrm{SrO} & 2.73 & 2.68 & 3.01 & 3.02 & 2.77 & 3.07 & 2.45 \\ \mathrm{BaO} & - & - & - & - & - & - & - \\ \mathrm{Na}{ }_{2} \mathrm{O} & 0.66 & 0.61 & 0.61 & 0.56 & 0.63 & 0.50 & 0.81 \\ \mathrm{~K}_{2} \mathrm{O} & 0.38 & 0.37 & 0.45 & 0.37 & 0.38 & 0.46 & 0.37 \\ \mathrm{Total} & 85.76 & 85.82 & 86.23 & 86.00 & 86.10 & 86.17 & 85.60 \\ & & & & & & & \\ & & & & & & & \\ \mathrm{Si} & 28.26 & 28.28 & 28.42 & 28.28 & 28.25 & 28.33 & 28.02 \\ \mathrm{Al} & 7.75 & 7.70 & 7.63 & 7.75 & 7.76 & 7.77 & 8.01 \\ \mathrm{Fe} & - & - & - & - & - & - & - \\ \mathrm{Mg} & 0.20 & 0.20 & - & 0.09 & 0.26 & - & 0.21 \\ \mathrm{Ca} & 2.53 & 2.59 & 2.52 & 2.57 & 2.49 & 2.53 & 2.63 \\ \mathrm{Sr} & 0.72 & 0.71 & 0.79 & 0.80 & 0.73 & 0.81 & 0.65 \\ \mathrm{Na} & 0.58 & 0.54 & 0.54 & 0.49 & 0.55 & 0.44 & 0.72 \\ \mathrm{~K} & 0.22 & 0.21 & 0.26 & 0.21 & 0.22 & 0.27 & 0.22 \\ \mathrm{Total} & 40.26 & 40.24 & 40.16 & 40.20 & 40.26 & 40.14 & 40.44 \\ \mathrm{O}^{-2} & 72.00 & 72.00 & 72.00 & 72.00 & 72.00 & 72.00 & 72.00 \\ \Sigma \text { cations } & 7.69 & 7.76 & 7.41 & 7.63 & 7.73 & 7.38 & 7.90 \\ & & & & & & & \\ \mathrm{Al} / \Sigma \text { cations } & 1.01 & 0.99 & 1.03 & 1.02 & 1.00 & 1.05 & 1.01 \\ \mathrm{Si} / \mathrm{Al} & 3.64 & 3.67 & 3.73 & 3.65 & 3.64 & 3.65 & 3.50 \\ \mathrm{Ca}+\mathrm{Mg}+\mathrm{Sr} & 3.44 & 3.50 & 3.31 & 3.46 & 3.48 & 3.34 & 3.48\end{array}$

UE-25a\#1 1242

\begin{tabular}{rrrrrrr}
\hline 62.23 & 61.59 & 64.42 & 63.21 & 63.00 & 61.42 & 63.94 \\
15.12 & 14.59 & 14.93 & 14.55 & 14.77 & 15.05 & 15.18 \\
- & - & - & - & - & - & - \\
0.12 & 0.08 & 0.76 & - & 0.05 & 0.17 & 0.07 \\
5.65 & 5.38 & 5.25 & 5.40 & 5.50 & 5.56 & 5.37 \\
2.41 & 2.49 & 1.99 & 2.53 & 2.36 & 2.47 & 2.35 \\
- & - & - & - & - & - & - \\
0.97 & 1.01 & 0.79 & 1.04 & 1.13 & 0.87 & 0.88 \\
0.45 & 0.39 & 0.39 & 0.57 & 0.49 & 0.40 & 0.50 \\
86.95 & 85.53 & 88.53 & 87.30 & 87.30 & 85.94 & 88.29 \\
& & & & & & \\
& & & & & & \\
28.00 & 28.15 & 28.25 & 28.31 & 28.20 & 27.96 & 28.22 \\
8.02 & 7.86 & 7.72 & 7.68 & 7.79 & 8.07 & 7.90 \\
- & - & - & - & - & - & - \\
0.08 & 0.05 & 0.50 & - & 0.03 & 0.12 & 0.05 \\
2.72 & 2.63 & 2.47 & 2.59 & 2.64 & 2.71 & 2.54 \\
0.63 & 0.66 & 0.51 & 0.66 & 0.61 & 0.65 & 0.60 \\
0.85 & 0.90 & 0.67 & 0.90 & 0.98 & 0.77 & 0.75 \\
0.26 & 0.23 & 0.22 & 0.33 & 0.28 & 0.23 & 0.28 \\
40.55 & 40.48 & 40.33 & 40.47 & 40.54 & 40.51 & 40.34 \\
72.00 & 72.00 & 72.00 & 72.00 & 72.00 & 72.00 & 72.00 \\
7.97 & 7.82 & 7.83 & 7.72 & 7.83 & 7.96 & 7.41 \\
& & & & & & \\
1.01 & 1.00 & 0.99 & 0.99 & 1.00 & 1.01 & 1.07 \\
3.49 & 3.58 & 3.66 & 3.69 & 3.62 & 3.46 & 3.57 \\
3.43 & 3.35 & 3.47 & 3.25 & 3.28 & 3.48 & 3.19
\end{tabular}


Tabular (large) Heulandite in the Devitrified Topopah Spring Tuff, continued

UE-25a\#1 1242

$\begin{array}{lrrrrrrr}\mathrm{SiO}_{2} & 63.04 & 63.11 & 61.04 & 62.16 & 61.04 & 61.06 & 62.09 \\ \mathrm{Al}_{2} \mathrm{O}_{3} & 14.74 & 14.85 & 14.12 & 14.04 & 14.40 & 14.90 & 14.74 \\ \mathrm{Fe}_{2} \mathrm{O}_{3} & - & - & - & - & - & - & - \\ \mathrm{MgO} & 0.10 & - & 0.26 & 0.13 & 0.23 & 0.45 & 0.13 \\ \mathrm{CaO} & 5.38 & 5.72 & 5.18 & 5.21 & 5.15 & 5.22 & 5.32 \\ \mathrm{SrO} & 2.33 & 2.54 & 2.07 & 2.14 & 2.70 & 2.45 & 2.42 \\ \mathrm{BaO} & - & - & - & - & - & - & - \\ \mathrm{Na}_{2} \mathrm{O} & 1.03 & 0.98 & 1.06 & 1.01 & 0.53 & 0.69 & 0.83 \\ \mathrm{~K}_{2} \mathrm{O} & 0.46 & 0.40 & 0.47 & 0.49 & 0.38 & 0.35 & 0.51 \\ \text { Total } & 87.08 & 87.60 & 84.20 & 85.18 & 84.43 & 85.12 & 86.04\end{array}$

G

\begin{tabular}{|c|c|c|c|c|c|c|c|}
\hline Si & 28.25 & 28.17 & 28.26 & 28.43 & $\begin{array}{r}28.22 \\
7.05\end{array}$ & 28.00 & $\begin{array}{r}28.18 \\
789\end{array}$ \\
\hline Al & 7.78 & 7.81 & 7.70 & 7.57 & 7.85 & 8.05 & 7.88 \\
\hline $\mathrm{Fe}$ & - & - & - & - & - & & \\
\hline $\mathrm{Mg}$ & 0.07 & - & 0.18 & 0.09 & 0.16 & 0.31 & 0.09 \\
\hline $\mathrm{Ca}$ & 2.58 & 2.74 & 2.57 & 2.55 & 2.55 & 2.56 & 2.59 \\
\hline $\mathrm{Sr}$ & 0.61 & 0.66 & 0.56 & 0.57 & 0.72 & 0.65 & 0.64 \\
\hline $\mathrm{Na}$ & 0.89 & 0.85 & 0.95 & 0.90 & 0.48 & 0.61 & 0.73 \\
\hline $\mathrm{K}$ & 0.26 & 0.23 & 0.28 & 0.29 & 0.22 & 0.20 & 0.30 \\
\hline Total & 40.44 & 40.46 & 40.50 & 40.38 & 40.20 & 40.39 & 40.40 \\
\hline $\mathrm{O}^{-2}$ & 72.00 & 72.00 & 72.00 & 72.00 & 72.00 & 72.00 & 72.00 \\
\hline ¿cations & 7.67 & 7.86 & 7.84 & 7.60 & 7.57 & 7.86 & 7.65 \\
\hline Al/scations & 1.02 & 0.99 & 0.98 & 1.00 & 1.04 & 1.02 & 1.03 \\
\hline $\mathrm{Si} / \mathrm{Al}$ & 3.63 & 3.61 & 3.67 & 3.76 & 3.60 & 3.48 & 3.57 \\
\hline $\mathrm{Ca}+\mathrm{Mg}+\mathrm{Sr}$ & 3.25 & 3.39 & 3.30 & 3.21 & 3.43 & 3.52 & 3.31 \\
\hline
\end{tabular}


Stellerite from the Devitrified Topopah Spring Tuff

\begin{tabular}{|c|c|c|c|c|c|c|c|c|}
\hline \multirow{3}{*}{$\begin{array}{l}\mathrm{SiO}_{2} \\
\mathrm{Al}_{2} \mathrm{O}_{3}\end{array}$} & \multicolumn{2}{|c|}{ USW G-2 1488} & \multicolumn{6}{|c|}{ USW G-2 1505} \\
\hline & 63.94 & 63.13 & 65.02 & 64.60 & 64.73 & 64.33 & 65.27 & 64.62 \\
\hline & 15.10 & 15.20 & 15.33 & 15.33 & 15.71 & 15.30 & 15.54 & 15.70 \\
\hline $\mathrm{Fe}_{2} \mathrm{O}_{3}$ & - & & - & - & - & - & - & \\
\hline $\mathrm{MgO}$ & - & - & - & - & - & - & - & \\
\hline $\mathrm{CaO}$ & 7.93 & 8.22 & 8.25 & 8.29 & 8.34 & 8.41 & 8.48 & 8.59 \\
\hline SrO & - & - & - & - & - & - & - & - \\
\hline $\mathrm{BaO}$ & - & - & - & - & - & - & - & - \\
\hline $\mathrm{Na}_{2} \mathrm{O}$ & 0.11 & 0.09 & 0.09 & 0.15 & 0.13 & - & - & 0.17 \\
\hline $\mathrm{K}_{2} \mathrm{O}$ & 0.17 & 0.08 & 0.12 & 0.08 & 0.10 & - & 0.08 & - \\
\hline Total & 87.25 & 86.72 & 88.81 & 88.45 & 89.01 & 88.04 & 89.37 & 89.08 \\
\hline $\mathrm{Si}$ & 28.19 & 28.04 & 28.18 & 28.12 & 28.02 & 28.12 & 28.12 & 27.97 \\
\hline $\mathrm{Al}$ & 7.85 & 7.96 & 7.83 & 7.87 & 8.01 & 7.88 & 7.89 & 8.01 \\
\hline $\mathrm{Fe}$ & - & - & - & - & - & - & - & - \\
\hline $\mathrm{Mg}$ & - & - & & - & - & - & - & \\
\hline $\mathrm{Ca}$ & 3.75 & 3.91 & 3.83 & 3.87 & 3.87 & 3.94 & 3.91 & 3.98 \\
\hline Sr & - & - & & - & - & - & - & - \\
\hline $\mathrm{Na}$ & 0.09 & 0.08 & 0.08 & 0.13 & 0.11 & - & - & 0.14 \\
\hline $\mathrm{K}$ & 0.10 & 0.05 & 0.07 & 0.04 & 0.06 & - & 0.04 & 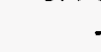 \\
\hline Total & 39.98 & 40.04 & 39.98 & 40.03 & 40.06 & 39.94 & 39.96 & 40.10 \\
\hline $\mathrm{O}^{-2}$ & 72.00 & 72.00 & 72.00 & 72.00 & 72.00 & 72.00 & 72.00 & 72.00 \\
\hline इcations & 7.68 & 7.95 & 7.80 & 7.90 & 7.90 & 7.88 & 7.87 & 8.11 \\
\hline $\mathrm{Al} / \Sigma$ cations & 1.02 & 1.00 & 1.00 & 1.00 & 1.01 & 1.00 & 1.00 & 0.99 \\
\hline $\mathrm{Si} / \mathrm{Al}$ & 3.59 & 3.52 & 3.60 & 3.58 & 3.50 & 3.57 & 3.56 & 3.49 \\
\hline $\mathrm{Ca}+\mathrm{Mg}+\mathrm{Sr}$ & 3.75 & 3.91 & 3.83 & 3.87 & 3.87 & 3.94 & 3.91 & 3.98 \\
\hline
\end{tabular}


Heulandite in the Basal Vitrophyre of the Topopah Spring Tuff

UE-25a\#1 1301

\begin{tabular}{|c|c|c|c|c|c|c|c|c|c|c|c|c|c|c|}
\hline $\mathrm{SiO}_{2}$ & 67.51 & 67.94 & 69.06 & 68.85 & 68.35 & 69.85 & 69.18 & 69.13 & 68.62 & 69.71 & 71.32 & 69.94 & 71.01 & 67.70 \\
\hline $\begin{array}{l}\mathrm{Al}_{2} \mathrm{O}_{3} \\
\mathrm{Fe}_{2} \mathrm{O}_{3}\end{array}$ & $\begin{array}{r}12.76 \\
-\end{array}$ & $\begin{array}{r}13.09 \\
-\end{array}$ & $\begin{array}{r}12.73 \\
-\end{array}$ & $\begin{array}{r}13.04 \\
-\end{array}$ & $\begin{array}{r}13.14 \\
-\end{array}$ & 12.81 & $\begin{array}{r}12.18 \\
-\end{array}$ & $\begin{array}{r}12.37 \\
-\end{array}$ & 12.40 & 13.34 & 13.10 & 12.46 & 12.55 & 13.01 \\
\hline $\mathrm{MgO}$ & 0.69 & 0.90 & 0.78 & 0.75 & 0.93 & 0.71 & 0.57 & 0.70 & 0.75 & 0.64 & 0.79 & 0.55 & 0.72 & 0.59 \\
\hline $\mathrm{CaO}$ & 4.03 & 4.04 & 3.85 & 4.17 & 3.98 & 3.90 & 3.69 & 3.64 & 3.66 & 4.18 & 3.88 & 3.79 & 3.61 & 4.09 \\
\hline SrO & 0.42 & 0.49 & 0.47 & 0.54 & 0.48 & 0.42 & 0.38 & 0.35 & 0.35 & 0.49 & 0.41 & 0.38 & 0.35 & 0.48 \\
\hline $\mathrm{BaO}$ & - & - & - & - & - & - & - & - & - & - & 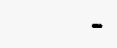 & - & - & \\
\hline $\mathrm{Na}_{2} \mathrm{O}$ & 0.83 & 0.68 & 0.79 & 0.79 & 0.82 & 0.80 & 1.06 & 0.81 & 0.86 & 0.80 & 0.84 & 1.07 & 0.95 & 0.82 \\
\hline $\mathrm{K}_{2} \mathrm{O}$ & 1.73 & 1.63 & 1.23 & 1.19 & 1.75 & 1.67 & 1.82 & 2.11 & 2.06 & 1.94 & 1.84 & 1.89 & 2.18 & 1.94 \\
\hline Total & 87.97 & 88.77 & 88.91 & 89.33 & 89.45 & 90.16 & 88.88 & 89.11 & 88.70 & 91.10 & 92.18 & 90.08 & 91.37 & 88.63 \\
\hline $\mathrm{Si}$ & 29.45 & 29.35 & 29.64 & 29.48 & 29.33 & 29.64 & 29.81 & 29.73 & 29.66 & 29.40 & 29.62 & 29.75 & 29.78 & 29.37 \\
\hline Al & 6.56 & 6.66 & 6.44 & 6.58 & 6.65 & 6.41 & 6.19 & 6.27 & 6.32 & 6.63 & 6.41 & 6.25 & 6.20 & 6.65 \\
\hline $\mathrm{Mg}$ & 0.45 & 0.58 & 0.50 & 0.48 & 0.59 & 0.45 & 0.37 & 0.45 & 0.48 & 0.40 & 0.49 & 0.35 & 0.45 & 0.38 \\
\hline $\mathrm{Ca}$ & 1.88 & 1.87 & 1.77 & 1.91 & 1.83 & 1.77 & 1.70 & 1.68 & 1.70 & 1.89 & 1.73 & 1.73 & 1.62 & 1.90 \\
\hline $\mathrm{Sr}$ & 0.11 & 0.12 & 0.12 & 0.13 & 0.12 & 0.10 & 0.09 & 0.09 & 0.09 & 0.12 & 0.10 & 0.09 & 0.09 & 0.12 \\
\hline $\mathrm{Na}$ & 0.70 & 0.57 & 0.66 & 0.66 & 0.68 & 0.66 & 0.89 & 0.68 & 0.72 & 0.65 & 0.68 & 0.88 & 0.77 & 0.69 \\
\hline$K$ & 0.96 & 0.90 & 0.67 & 0.65 & 0.96 & 0.90 & 1.00 & 1.16 & 1.14 & 1.04 & 0.5 & 1. & & 1.07 \\
\hline Total & 40.11 & 40.05 & 39.80 & 39.89 & 40.16 & 39.94 & 40.04 & 40.05 & 40.11 & 40.14 & 40.00 & 40.08 & 40.08 & 40.19 \\
\hline $\mathrm{O}^{-2}$ & 72.00 & 72.00 & 72.00 & 72.00 & 72.00 & 72.00 & 72.00 & 72.00 & 72.00 & 72.00 & 72.00 & 72.00 & 72.00 & 72.00 \\
\hline$\Sigma$ cations & 6.54 & 6.61 & 6.10 & 6.36 & 6.73 & 6.21 & 6.21 & 6.26 & 6.39 & 6.52 & 6.28 & 6.25 & 6.25 & 6.57 \\
\hline $\mathrm{Al} / \Sigma \mathrm{ca}$ & 1.00 & 1.0 & & & & & 1. & & 0. & & 1. & & 19 & 1.01 \\
\hline Si/AI & 4.49 & 4.4 & 4.6 & 4.4 & & 4. & & & 4.70 & & 4. & 4. & 30 & 4.42 \\
\hline $\mathrm{Ca}+\mathrm{Mg}+\mathrm{Sr}$ & 2.44 & 2.57 & 2.39 & 2.53 & 2.54 & 2.33 & 2.16 & 2.21 & 2.27 & 2.41 & 2.31 & 2.17 & 2.16 & 2.40 \\
\hline
\end{tabular}


Heulandite in the Basal Vitrophyre of the Topopah Spring Tuff, continued

UE-25a\#1 1301

\begin{tabular}{|c|c|c|c|c|c|c|c|c|c|c|c|c|c|c|}
\hline $\mathrm{SiO}_{2}$ & 68.69 & 70.01 & 69.84 & 70.92 & 68.23 & 68.98 & 70.55 & 69.68 & 70.83 & 71.42 & 71.24 & 69.73 & 71.59 & 72.49 \\
\hline $\begin{array}{l}\mathrm{Al}_{2} \mathrm{O}_{3} \\
\mathrm{Fe}_{2} \mathrm{O}_{3}\end{array}$ & $\begin{array}{r}13.25 \\
-\end{array}$ & $\begin{array}{r}13.14 \\
-\end{array}$ & $\begin{array}{r}13.43 \\
-\end{array}$ & $\begin{array}{r}12.73 \\
-\end{array}$ & $\begin{array}{r}13.54 \\
-\end{array}$ & $\begin{array}{r}12.96 \\
-\end{array}$ & $\begin{array}{r}13.96 \\
-\end{array}$ & $\begin{array}{r}13.89 \\
-\end{array}$ & $\begin{array}{r}13.92 \\
-\end{array}$ & $\begin{array}{r}13.35 \\
-\end{array}$ & $\begin{array}{r}13.75 \\
-\end{array}$ & $\begin{array}{r}13.73 \\
-\end{array}$ & 13.29 & 13.33 \\
\hline $\mathrm{MgO}$ & 0.87 & 0.85 & 0.57 & 0.75 & 0.84 & 0.86 & 0.69 & 0.92 & 0.73 & 0.81 & 0.67 & 0.92 & 0.66 & 0.72 \\
\hline $\mathrm{CaO}$ & 4.09 & 4.06 & 4.11 & 3.78 & 4.38 & 4.02 & 4.56 & 4.37 & 4.54 & 3.98 & 4.39 & 4.08 & 4.04 & 4.07 \\
\hline SrO & 0.46 & 0.41 & 0.48 & 0.38 & 0.47 & 0.39 & 0.55 & 0.47 & 0.53 & 0.38 & 0.52 & 0.46 & 0.43 & 0.43 \\
\hline $\mathrm{BaO}$ & - & - & - & & & - & & & & - & - & - & - & - \\
\hline $\mathrm{Na}_{2} \mathrm{O}$ & 0.76 & 0.77 & 0.88 & 0.86 & 0.63 & 0.72 & 0.79 & 0.82 & 0.78 & 0.85 & 0.91 & 0.72 & 0.88 & 0.79 \\
\hline $\mathrm{K}_{2} \mathrm{O}$ & 1.86 & 1.97 & 1.87 & 1.86 & 1.24 & 1.61 & 1.33 & 1.51 & 1.42 & 1.53 & 1.28 & 1.57 & 1.61 & 1.74 \\
\hline Total & 89.98 & 91.21 & 91.18 & 91.28 & 89.33 & 89.54 & 92.43 & 91.66 & 92.75 & 92.32 & 92.76 & 91.21 & 92.50 & 93.57 \\
\hline Si & 29.32 & 29.46 & 29.41 & 29.73 & 29.24 & 29.49 & 29.26 & 29.17 & 29.28 & 29.56 & 29.40 & 29.28 & 29.60 & 29.64 \\
\hline $\mathrm{Al}$ & 6.67 & 6.52 & 6.66 & 6.29 & 6.84 & 6.53 & 6.82 & 6.85 & 6.78 & 6.51 & 6.69 & 6.80 & 6.48 & 6.42 \\
\hline $\mathrm{Mg}$ & 0.55 & 0.53 & 0.36 & 0.47 & 0.54 & 0.55 & 0.43 & 0.57 & 0.45 & 0.50 & 0.41 & 0.58 & 0.41 & 0.44 \\
\hline $\mathrm{Ca}$ & 1.87 & 1.83 & 1.85 & 1.70 & 2.01 & 1.84 & 2.03 & 1.96 & 2.01 & 1.77 & 1.94 & 1.84 & 1.79 & 1.78 \\
\hline $\mathrm{Sr}$ & 0.11 & 0.10 & 0.12 & 0.09 & 0.12 & 0.10 & 0.13 & 0.11 & 0.13 & 0.09 & 0.12 & 0.11 & 0.10 & 0.10 \\
\hline $\mathrm{Na}$ & 0.63 & 0.63 & 0.72 & 0.70 & 0.52 & 0.60 & 0.64 & 0.67 & 0.63 & 0.68 & 0.73 & 0.59 & 0.71 & 0.63 \\
\hline K & 1.01 & 1.06 & 1.00 & 0.99 & 0.68 & 0.88 & 0.70 & 0.81 & 0.75 & 0.81 & 0.67 & 0.84 & 0.85 & 0.91 \\
\hline Total & 40.17 & 40.13 & 40.12 & 39.97 & 39.94 & 39.98 & 40.00 & 40.14 & 40.02 & 39.92 & 39.96 & 40.03 & 39.94 & 39.92 \\
\hline $0^{-2}$ & 72.00 & 72.00 & 72.00 & 72.00 & 72.00 & 72.00 & 72.00 & 72.00 & 72.00 & 72.00 & 72.00 & 72.00 & 72.00 & 72.00 \\
\hline$\Sigma$ cations & 6.72 & 6.61 & 6.38 & 6.21 & 6.53 & 6.45 & 6.51 & 6.77 & 6.55 & 6.20 & 6.36 & 6.48 & 6.15 & 6.18 \\
\hline Al/scations & 0.99 & 0.99 & 1.04 & 1.01 & 1.05 & 1.01 & 1.05 & 1.01 & 1.04 & 1.05 & 1.05 & 1.05 & 1.05 & 1.04 \\
\hline Si/Al & 4.40 & 4.52 & 4.41 & 4.73 & 4.28 & 4.52 & 4.29 & 4.26 & 4.32 & 4.54 & 4.40 & 4.31 & 4.57 & 4.61 \\
\hline $\mathrm{Ca}+\mathrm{Mg}+\mathrm{Sr}$ & 2.54 & 2.46 & 2.33 & 2.26 & 2.66 & 2.49 & 2.58 & 2.65 & 2.59 & 2.36 & 2.48 & 2.52 & 2.30 & 2.32 \\
\hline
\end{tabular}


Phillipsite in the Basal Vitrophyre of the Topopah Spring Tuff

\section{USW GU-3 1201}

\begin{tabular}{|c|c|c|c|c|c|c|c|c|c|c|c|c|c|c|c|}
\hline $\begin{array}{l}\mathrm{SiO}_{2} \\
\mathrm{Al}_{2} \mathrm{O}_{3}\end{array}$ & $\begin{array}{l}64.79 \\
16.58\end{array}$ & $\begin{array}{l}67.05 \\
15.91\end{array}$ & $\begin{array}{l}64.49 \\
17.08\end{array}$ & $\begin{array}{l}65.33 \\
16.11\end{array}$ & $\begin{array}{l}64.77 \\
16.49\end{array}$ & $\begin{array}{l}64.96 \\
16.13\end{array}$ & $\begin{array}{l}64.24 \\
16.44\end{array}$ & $\begin{array}{l}65.31 \\
16.59\end{array}$ & $\begin{array}{l}64.44 \\
16.73\end{array}$ & $\begin{array}{l}65.43 \\
16.70\end{array}$ & $\begin{array}{l}64.60 \\
16.24\end{array}$ & $\begin{array}{l}63.45 \\
16.47\end{array}$ & $\begin{array}{l}63.81 \\
16.49\end{array}$ & $\begin{array}{l}64.00 \\
16.58\end{array}$ & $\begin{array}{l}64.6 \\
16.4\end{array}$ \\
\hline $\mathrm{Fe}_{2} \mathrm{O}_{3}$ & - & 0.20 & - & & - & - & 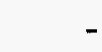 & 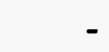 & - & - & - & - & - & 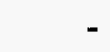 & \\
\hline $\mathrm{MgO}$ & - & 0.06 & - & 0.12 & - & - & - & - & - & - & - & - & - & - & \\
\hline $\mathrm{CaO}$ & 5.09 & 5.20 & 6.82 & 6.96 & 4.81 & 5.14 & 5.24 & 4.99 & 6.08 & 6.43 & 5.17 & 6.21 & 5.80 & 5.16 & \\
\hline SrO & 0.24 & 0.67 & 0.22 & 0.28 & 0.22 & 0.21 & 0.18 & 0.24 & 0.26 & 0.24 & 0.18 & 0.21 & 0.31 & 0.16 & \\
\hline $\mathrm{BaO}$ & 0.10 & - & 0.10 & - & - & - & & 0.10 & - & - & - & 0.15 & - & - & \\
\hline $\mathrm{Na}_{2} \mathrm{O}$ & 2.15 & 1.48 & 1.23 & 0.95 & 2.14 & 2.25 & 2.36 & 2.13 & 1.80 & 1.47 & 2.11 & 1.35 & 1.76 & 2.26 & \\
\hline $\mathrm{K}_{2} \mathrm{O}$ & 2.63 & 2.20 & 1.61 & 1.20 & 3.18 & 2.73 & 2.91 & 2.88 & 2.38 & 1.97 & 2.28 & 2.06 & 2.26 & 2.68 & \\
\hline Total & 91.58 & 92.77 & 91.55 & 90.95 & 91.61 & 91.42 & 91.37 & 92.24 & 91.69 & 92.24 & 90.58 & 89.90 & 90.43 & 90.84 & \\
\hline $\mathrm{Si}$ & 12.32 & 12.52 & 12.22 & 12.40 & 12.33 & 12.37 & 12.28 & 12.34 & 12.25 & 12.31 & 12.37 & 12.27 & 12.28 & 12.28 & \\
\hline$A l$ & 3.72 & 3.50 & 3.81 & 3.60 & 3.70 & 3.62 & 3.70 & 3.69 & 3.75 & 3.70 & 3.67 & 3.75 & 3.74 & 3.75 & \\
\hline $\mathrm{Fe}$ & - & 0.03 & - & - & - & - & - & - & - & - & - & - & - & - & \\
\hline Mg & - & 0.02 & - & 0.03 & - & - & - & - & - & - & - & - & - & - & \\
\hline $\mathrm{Ca}$ & 1.04 & 1.04 & 1.38 & 1.42 & 0.98 & 1.05 & 1.07 & 1.01 & 1.24 & 1.30 & 1.06 & 1.29 & 1.20 & 1.06 & \\
\hline Sr & 0.03 & 0.07 & 0.02 & 0.03 & 0.02 & 0.02 & 0.02 & 0.03 & 0.03 & 0.03 & 0.02 & 0.02 & 0.03 & 0.02 & \\
\hline $\mathrm{Ba}$ & 0.01 & - & 0.01 & - & - & - & - & 0.01 & - & - & - & 0.01 & - & - & \\
\hline $\mathrm{Na}$ & 0.79 & 0.54 & 0.45 & 0.35 & 0.79 & 0.83 & 0.87 & 0.78 & 0.66 & 0.54 & 0.78 & 0.51 & 0.66 & 0.84 & \\
\hline K & 0.64 & 0.52 & 0.39 & 0.29 & 0.77 & 0.66 & 0.71 & 0.69 & 0.58 & 0.47 & 0.56 & 0.51 & 0.55 & 0.66 & \\
\hline Total & 18.54 & 18.24 & 18.29 & 18.12 & 18.60 & 18.56 & 18.66 & 18.55 & 18.50 & 18.34 & 18.46 & 18.36 & 18.46 & 18.60 & 18. \\
\hline $0^{-2}$ & 32.00 & 32.00 & 32.00 & 32.00 & 32.00 & 32.00 & 32.00 & 32.00 & 32.00 & 32.00 & 32.00 & 32.00 & 32.00 & 32.00 & 32. \\
\hline Scations & 3.57 & 3.32 & 3.67 & 3.60 & 3.57 & 3.64 & 3.77 & 3.56 & 3.77 & 3.65 & 3.50 & 3.66 & 3.67 & 3.65 & \\
\hline $\mathrm{Al} / \Sigma$ cations & 1.04 & 1.05 & 1.04 & 1.00 & 1.04 & 1.00 & 0.98 & 1.04 & 0.99 & 1.01 & 1.05 & 1.03 & 1.02 & 1.03 & \\
\hline Si/Al & 3.32 & 3.58 & 3.20 & 3.44 & 3.33 & 3.42 & 3.32 & 3.34 & 3.27 & 3.32 & 3.38 & 3.27 & 3.28 & 3.28 & \\
\hline $\mathrm{Ca}+\mathrm{Mg}+\mathrm{Sr}$ & 1.06 & 1.13 & 1.41 & 1.48 & 1.01 & 1.07 & 1.09 & 1.04 & 1.27 & 1.32 & 1.08 & 1.31 & 1.23 & 1.08 & \\
\hline
\end{tabular}


Phillipsite in the Basal Vitrophyre of the Topopah Spring Tuff, continued

USW GU-3 1201

\begin{tabular}{lrrrrrrr} 
& & & & & & & \\
$\mathrm{SiO}_{2}$ & 63.60 & 64.14 & 63.23 & 62.01 & 61.90 & 62.62 & 61.10 \\
$\mathrm{Al}_{2} \mathrm{O}_{3}$ & 16.47 & 15.73 & 16.18 & 15.44 & 15.27 & 15.54 & 15.39 \\
$\mathrm{Fe}_{2} \mathrm{O}_{3}$ & - & 0.05 & 0.27 & - & - & - & - \\
$\mathrm{MgO}$ & - & 0.05 & - & - & - & - & - \\
$\mathrm{CaO}$ & 5.59 & 4.62 & 4.84 & 4.87 & 4.63 & 4.72 & 5.55 \\
$\mathrm{SrO}$ & 0.31 & 0.32 & 0.38 & 0.24 & 0.25 & 0.32 & 0.39 \\
$\mathrm{BaO}$ & 0.10 & 0.08 & - & - & - & 0.08 & 0.07 \\
$\mathrm{Na} \mathrm{O}_{2} \mathrm{O}$ & 1.55 & 2.11 & 2.22 & 2.07 & 2.05 & 2.10 & 1.26 \\
$\mathrm{~K}_{2} \mathrm{O}$ & 2.65 & 2.78 & 3.04 & 2.95 & 3.08 & 2.69 & 2.18 \\
$\mathrm{Total}$ & 90.27 & 89.88 & 90.16 & 87.58 & 87.18 & 88.07 & 85.94 \\
& & & & & & & \\
$\mathrm{Si}$ & 12.28 & 12.42 & 12.27 & 12.36 & 12.39 & 12.39 & 12.36 \\
$\mathrm{Al}$ & 3.75 & 3.59 & 3.70 & 3.63 & 3.60 & 3.62 & 3.67 \\
$\mathrm{Fe}$ & - & 0.01 & 0.04 & - & - & - & - \\
$\mathrm{Mg}$ & - & 0.01 & - & - & - & - & - \\
$\mathrm{Ca}$ & 1.16 & 0.96 & 1.01 & 1.04 & 0.99 & 1.00 & 1.20 \\
$\mathrm{Sr}$ & 0.03 & 0.04 & 0.04 & 0.03 & 0.03 & 0.04 & 0.05 \\
$\mathrm{Ba}$ & 0.01 & 0.01 & - & - & - & 0.01 & 0.01 \\
$\mathrm{Na}$ & 0.58 & 0.79 & 0.84 & 0.80 & 0.80 & 0.81 & 0.49 \\
$\mathrm{~K}$ & 0.65 & 0.69 & 0.75 & 0.75 & 0.79 & 0.68 & 0.56 \\
$\mathrm{Total}$ & 18.46 & 18.52 & 18.65 & 18.60 & 18.60 & 18.54 & 18.34 \\
$\mathrm{O}$ & 32.00 & 32.00 & 32.00 & 32.00 & 32.00 & 32.00 & 32.00 \\
$\mathrm{\Sigma}$ & 3.63 & 3.51 & 3.69 & 3.69 & 3.63 & 3.57 & 3.56 \\
& & & & & & & \\
$\mathrm{Al} / \mathrm{L}$ cations & 1.03 & 1.02 & 1.00 & 0.98 & 0.99 & 1.01 & 1.03 \\
$\mathrm{Si} / \mathrm{Al}$ & 3.28 & 3.46 & 3.32 & 3.41 & 3.44 & 3.42 & 3.37 \\
$\mathrm{Ca}+\mathrm{Mg}+\mathrm{Sr}$ & 1.19 & 1.01 & 1.05 & 1.07 & 1.02 & 1.04 & 1.25 \\
& & & & & & & \\
\hline & & & & & & &
\end{tabular}


APPENDIX III

FRACTURE-LINING MANGANESE-OXIDE MINERALS IN THE PAINTBRUSH GROUP

Quantitative analyses of rancieite were accepted if $\mathrm{SiO}_{2}$ was $<2 \mathrm{wt} \%$ and $\mathrm{Al}_{2} \mathrm{O}_{3}$ was $<12$ wt\%.

Semiquantitative analyses (on unpolished surfaces) were accepted:

for rancieite if $\mathrm{SiO}_{2}$ was $<2 \mathrm{wt} \%$ and $\mathrm{Al}_{2} \mathrm{O}_{3}$ was $<12 \mathrm{wt} \%$, and

for lithiophorite if $\mathrm{SiO}_{2}$ was $<4 \mathrm{wt} \%$ and $\mathrm{Al}_{2} \mathrm{O}_{3}$ was $>18 \mathrm{wt} \%$, and if totals were $>75 \%$.

Sample numbers indicate drill hole and depth in feet.

$\begin{aligned} \mathrm{n} / \mathrm{a} & =\text { not analyzed } \\ - & =\text { not detected }\end{aligned}$ 
USW G-4 49.9

\begin{tabular}{|c|c|c|c|c|c|}
\hline $\mathrm{SiO}_{2}$ & 1.42 & 1.33 & 1.22 & 1.02 & 1.14 \\
\hline $\mathrm{TiO}_{2}$ & 0.67 & 0.78 & 0.59 & 1.22 & 1.32 \\
\hline $\mathrm{MnO}_{2}$ & 58.34 & 59.30 & 61.01 & 58.93 & 61.61 \\
\hline $\mathrm{CeO}_{2}$ & 4.28 & 2.87 & 1.53 & 1.59 & 1.71 \\
\hline $\mathrm{Al}_{2} \mathrm{O}_{3}$ & 10.22 & 8.20 & 11.31 & 10.86 & 10.69 \\
\hline $\mathrm{Fe}_{2} \mathrm{O}_{3}$ & 1.57 & 2.56 & 1.19 & 2.30 & 2.71 \\
\hline $\mathrm{MgO}$ & 1.17 & 1.18 & 1.26 & 0.93 & 1.00 \\
\hline $\mathrm{CaO}$ & 2.66 & 3.53 & 2.47 & 3.35 & 3.54 \\
\hline $\mathrm{NiO}$ & - & - & - & 0.11 & - \\
\hline CuO & - & 0.23 & 0.21 & 0.38 & - \\
\hline $\mathrm{ZnO}$ & 0.91 & 0.87 & 1.08 & 1.19 & 0.70 \\
\hline SrO & 0.14 & 0.14 & 0.06 & 0.10 & - \\
\hline $\mathrm{BaO}$ & 1.29 & 1.12 & 0.78 & 0.69 & 0.86 \\
\hline $\mathrm{PbO}$ & 3.75 & 3.20 & 4.16 & 4.03 & 2.45 \\
\hline $\mathrm{Na}_{2} \mathrm{O}$ & - & 0.12 & 0.09 & - & - \\
\hline $\mathrm{K}_{2} \mathrm{O}$ & 0.20 & 0.15 & 0.16 & 0.14 & 0.10 \\
\hline $\mathrm{P}_{2} \mathrm{O}_{5}$ & 0.22 & 0.17 & 0.07 & - & - \\
\hline $\mathrm{La}_{2} \mathrm{O}_{3}$ & - & - & - & 0.13 & - \\
\hline $\mathrm{MoO}_{3}$ & - & - & - & - & - \\
\hline $\mathrm{WO}_{3}$ & 0.43 & 0.28 & 0.29 & 0.39 & 0.33 \\
\hline $\mathrm{Cl}$ & 0.08 & 0.11 & 0.10 & 0.08 & 0.08 \\
\hline Total & 87.35 & 86.14 & 87.58 & 87.44 & 88.24 \\
\hline $\mathrm{Si}$ & 0.11 & 0.11 & 0.09 & 0.08 & 0.09 \\
\hline $\mathrm{Ti}$ & 0.04 & 0.05 & 0.03 & 0.07 & 0.07 \\
\hline $\mathrm{Mn}$ & 3.15 & 3.24 & 3.23 & 3.15 & 3.20 \\
\hline $\mathrm{Ce}$ & 0.12 & 0.08 & 0.04 & 0.04 & 0.04 \\
\hline Al & 0.94 & 0.76 & 1.02 & 0.99 & 0.95 \\
\hline $\mathrm{Fe}$ & 0.09 & 0.15 & 0.07 & 0.13 & 0.15 \\
\hline $\mathrm{Mg}$ & 0.14 & 0.14 & 0.14 & 0.11 & 0.11 \\
\hline $\mathrm{Ca}$ & 0.22 & 0.30 & 0.20 & 0.28 & 0.29 \\
\hline $\mathrm{Ni}$ & - & - & - & 0.01 & - \\
\hline $\mathrm{Cu}$ & - & 0.01 & 0.01 & 0.02 & - \\
\hline $\mathrm{Zn}$ & 0.05 & 0.05 & 0.06 & 0.07 & 0.04 \\
\hline $\mathrm{Sr}$ & 0.01 & 0.01 & 0.00 & 0.00 & - \\
\hline $\mathrm{Ba}$ & 0.04 & 0.03 & 0.02 & 0.02 & 0.03 \\
\hline $\mathrm{Pb}$ & 0.08 & 0.07 & 0.09 & 0.08 & 0.05 \\
\hline $\mathrm{Na}$ & - & 0.02 & 0.01 & - & - \\
\hline $\mathrm{K}$ & 0.02 & 0.02 & 0.02 & 0.01 & 0.01 \\
\hline$P$ & 0.01 & 0.01 & 0.00 & - & - \\
\hline La & - & - & - & 0.00 & - \\
\hline Mo & - & - & - & - & - \\
\hline$W$ & 0.01 & 0.01 & 0.01 & 0.01 & 0.01 \\
\hline Total & 5.03 & 5.06 & 5.06 & 5.08 & 5.03 \\
\hline $\mathrm{O}^{-2}$ & 9.00 & 9.00 & 9.00 & 9.00 & 9.00 \\
\hline
\end{tabular}

USW G-4 64.5

\begin{tabular}{rrrrrr}
\hline 1.37 & 1.32 & 1.21 & 1.41 & 1.04 & 1.88 \\
1.07 & 0.51 & 0.62 & 0.13 & - & 1.07 \\
59.44 & 60.19 & 60.54 & 60.22 & 62.45 & 56.94 \\
3.77 & 4.88 & 3.45 & 4.34 & 2.87 & 8.06 \\
10.19 & 8.44 & 8.42 & 5.32 & 6.28 & 8.59 \\
2.29 & 0.89 & 1.15 & 2.36 & 1.24 & 1.78 \\
1.14 & 1.09 & 1.20 & 0.93 & 1.17 & 1.26 \\
3.50 & 3.22 & 3.06 & 3.53 & 2.19 & 3.26 \\
0.25 & - & 0.13 & 0.12 & - & 0.16 \\
- & - & 0.23 & 0.24 & - & 0.34 \\
0.69 & 0.53 & 0.51 & 0.73 & 0.60 & 0.68 \\
- & 0.10 & 0.10 & 0.17 & 0.23 & 0.07 \\
0.78 & 1.39 & 1.23 & 1.43 & 1.73 & 0.92 \\
3.20 & 3.07 & 4.16 & 3.31 & 5.44 & 2.29 \\
- & - & - & 0.08 & - & - \\
0.14 & 0.14 & 0.13 & 0.14 & 0.23 & 0.09 \\
0.17 & 0.19 & 0.15 & 0.23 & 0.15 & 0.22 \\
- & - & - & 0.14 & 0.18 & 0.09 \\
- & - & - & - & - & - \\
0.37 & 0.45 & 0.38 & 0.46 & 0.45 & - \\
0.12 & 0.07 & 0.11 & 0.08 & 0.06 & 0.17 \\
88.49 & 86.48 & 86.78 & 85.37 & 86.31 & 87.87
\end{tabular}

$\begin{array}{llllll}0.10 & 0.10 & 0.10 & 0.12 & 0.08 & 0.15 \\ 0.06 & 0.03 & 0.04 & 0.01 & - & 0.06 \\ 3.14 & 3.29 & 3.31 & 3.41 & 3.51 & 3.09 \\ 0.10 & 0.13 & 0.10 & 0.12 & 0.08 & 0.22 \\ 0.92 & 0.79 & 0.79 & 0.51 & 0.60 & 0.79 \\ 0.13 & 0.05 & 0.07 & 0.15 & 0.08 & 0.11 \\ 0.13 & 0.13 & 0.14 & 0.11 & 0.14 & 0.15 \\ 0.29 & 0.27 & 0.26 & 0.31 & 0.19 & 0.27 \\ 0.02 & - & 0.01 & 0.01 & - & 0.01 \\ - & - & 0.01 & 0.01 & - & 0.02 \\ 0.04 & 0.03 & 0.03 & 0.04 & 0.04 & 0.04 \\ - & 0.00 & 0.00 & 0.01 & 0.01 & 0.00 \\ 0.02 & 0.04 & 0.04 & 0.05 & 0.06 & 0.03 \\ 0.07 & 0.07 & 0.09 & 0.07 & 0.12 & 0.05 \\ - & - & - & 0.01 & - & - \\ 0.01 & 0.01 & 0.01 & 0.01 & 0.02 & 0.01 \\ 0.01 & 0.01 & 0.01 & 0.02 & 0.01 & 0.01 \\ - & - & - & 0.00 & 0.01 & 0.00 \\ - & - & - & - & - & - \\ 0.01 & 0.01 & 0.01 & 0.01 & 0.01 & - \\ 5.05 & 4.99 & 5.01 & 4.99 & 4.96 & 5.01 \\ 9.00 & 9.00 & 9.00 & 9.00 & 9.00 & 9.00\end{array}$


Rancieite in the Tiva Canyon Tuff, continued

\begin{tabular}{|c|c|c|c|c|c|}
\hline \multirow[b]{2}{*}{$\mathrm{SiO}_{2}$} & \multicolumn{5}{|c|}{ USW G-4 70.85} \\
\hline & 1.12 & 0.99 & 0.60 & 1.26 & 0.71 \\
\hline $\mathrm{TiO}_{2}$ & 0.64 & 0.52 & - & 0.61 & - \\
\hline $\mathrm{MnO}_{2}$ & 62.81 & 61.22 & 61.90 & 57.47 & 64.07 \\
\hline $\mathrm{CeO}_{2}$ & 4.41 & 2.95 & 0.94 & 3.33 & 1.47 \\
\hline $\mathrm{Al}_{2} \mathrm{O}_{3}$ & 11.74 & 10.52 & 11.73 & 11.01 & 7.92 \\
\hline $\mathrm{Fe}_{2} \mathrm{O}_{3}$ & 1.22 & 2.04 & 0.57 & 2.22 & 1.34 \\
\hline $\mathrm{MgO}$ & 1.35 & 1.23 & 1.45 & 1.12 & 1.20 \\
\hline $\mathrm{CaO}$ & 2.42 & 1.94 & 2.09 & 2.43 & 2.17 \\
\hline $\mathrm{NiO}$ & - & - & - & 0.23 & 0.18 \\
\hline $\mathrm{CuO}$ & 0.51 & 0.43 & 0.59 & 0.61 & 0.74 \\
\hline $\mathrm{ZnO}$ & 1.04 & 0.84 & 1.14 & 0.95 & 1.17 \\
\hline SrO & 0.09 & 0.09 & 0.15 & 0.05 & 0.27 \\
\hline $\mathrm{BaO}$ & 0.68 & 0.81 & 0.72 & 0.65 & 1.11 \\
\hline $\mathrm{PbO}$ & 3.57 & 4.57 & 4.24 & 2.98 & 6.27 \\
\hline $\mathrm{Na}_{2} \mathrm{O}$ & 0.12 & 0.12 & 0.08 & 0.17 & 0.25 \\
\hline $\mathrm{K}_{2} \mathrm{O}$ & 0.12 & 0.14 & 0.20 & 0.11 & 0.23 \\
\hline $\mathrm{P}_{2} \mathrm{O}_{5}$ & 0.12 & 0.14 & 0.08 & 0.15 & 0.11 \\
\hline $\mathrm{La}_{2} \mathrm{O}_{3}$ & - & - & 0.15 & 0.19 & 0.33 \\
\hline $\mathrm{MoO}_{3}$ & - & - & 0.22 & - & - \\
\hline $\mathrm{WO}_{3}$ & 0.39 & - & 0.58 & 0.44 & 0.73 \\
\hline $\mathrm{Cl}$ & 0.06 & 0.05 & 0.06 & 0.08 & 0.07 \\
\hline Total & 92.41 & 88.60 & 87.49 & 86.06 & 90.34 \\
\hline Si & 0.08 & 0.08 & 0.05 & 0.10 & 0.06 \\
\hline $\mathrm{Ti}$ & 0.04 & 0.03 & - & 0.04 & - \\
\hline $\mathrm{Mn}$ & 3.18 & 3.25 & 3.29 & 3.11 & 3.44 \\
\hline $\mathrm{Ce}$ & 0.11 & 0.08 & 0.03 & 0.09 & 0.04 \\
\hline $\mathrm{Al}$ & 1.01 & 0.95 & 1.06 & 1.02 & 0.73 \\
\hline $\mathrm{Fe}$ & 0.07 & 0.12 & 0.03 & 0.13 & 0.08 \\
\hline $\mathrm{Mg}$ & 0.15 & 0.14 & 0.17 & 0.13 & 0.14 \\
\hline $\mathrm{Ca}$ & 0.19 & 0.16 & 0.17 & 0.20 & 0.18 \\
\hline $\mathrm{Ni}$ & - & - & - & 0.01 & 0.01 \\
\hline $\mathrm{Cu}$ & 0.03 & 0.02 & 0.03 & 0.04 & 0.04 \\
\hline $\mathrm{Zn}$ & 0.06 & 0.05 & 0.06 & 0.05 & 0.07 \\
\hline $\mathrm{Sr}$ & 0.00 & 0.00 & 0.01 & 0.00 & 0.01 \\
\hline $\mathrm{Ba}$ & 0.02 & 0.02 & 0.02 & 0.02 & 0.03 \\
\hline $\mathrm{Pb}$ & 0.07 & 0.09 & 0.09 & 0.06 & 0.13 \\
\hline $\mathrm{Na}$ & 0.02 & 0.02 & 0.01 & 0.03 & 0.04 \\
\hline $\mathrm{K}$ & 0.01 & 0.01 & 0.02 & 0.01 & 0.02 \\
\hline$P$ & 0.01 & 0.01 & 0.01 & 0.01 & 0.01 \\
\hline La & - & - & 0.00 & 0.01 & 0.01 \\
\hline Mo & - & - & 0.01 & - & - \\
\hline W & 0.01 & - & 0.01 & 0.01 & 0.01 \\
\hline Total & 5.04 & 5.04 & 5.06 & 5.07 & 5.05 \\
\hline $\mathrm{O}^{-2}$ & 9.00 & 9.00 & 9.00 & 9.00 & 9.00 \\
\hline
\end{tabular}


Semiquantitative Analyses (on Unpolished Flat Surfaces) of Rancieite and Lithiophorite in the Topopah Spring Tuff

\begin{tabular}{|c|c|c|c|c|c|c|c|c|c|c|}
\hline \multirow{8}{*}{$\begin{array}{l}\mathrm{SiO}_{2} \\
\mathrm{TiO}_{2} \\
\mathrm{MnO}_{2} \\
\mathrm{Al}_{2} \mathrm{O}_{3} \\
\mathrm{Fe}_{2} \mathrm{O}_{3} \\
\mathrm{MgO}\end{array}$} & \multicolumn{3}{|c|}{ Rancieite } & \multicolumn{7}{|c|}{ Lithiophorite } \\
\hline & \multirow{2}{*}{$\begin{array}{c}\text { USW G-4 } \\
795.6 \\
\\
1.55\end{array}$} & \multicolumn{2}{|c|}{$\begin{array}{c}\text { UE-25a\#1 } \\
1252.3\end{array}$} & \multicolumn{2}{|c|}{$\begin{array}{c}\text { UE-25a\#1 } \\
1252.3\end{array}$} & \multicolumn{4}{|c|}{$\begin{array}{c}\text { USW G-4 } \\
1258.0\end{array}$} & \multirow{2}{*}{$\begin{array}{l}\text { USW G-4 } \\
\begin{array}{r}1201.6 \\
\\
3.35\end{array}\end{array}$} \\
\hline & & 1.29 & 1.96 & 1.00 & 1.31 & 1.08 & 3.60 & 1.96 & 3.96 & \\
\hline & 0.94 & 1.54 & 1.54 & 0.65 & 0.55 & 0.12 & 0.33 & 0.44 & 0.66 & 0.54 \\
\hline & 60.85 & 66.06 & 64.59 & 50.29 & 49.39 & 49.45 & 47.93 & 50.62 & 50.96 & 53.99 \\
\hline & 7.03 & 2.54 & 3.62 & 19.16 & 20.97 & 23.77 & 22.66 & 18.77 & 23.40 & 20.60 \\
\hline & 2.02 & 5.08 & 4.54 & 2.96 & 2.57 & 0.37 & 1.01 & 1.78 & 1.63 & 2.56 \\
\hline & 0.74 & 0.66 & 0.59 & 0.68 & 0.93 & 0.64 & 0.58 & 0.49 & 0.47 & 0.85 \\
\hline $\mathrm{CaO}$ & 4.84 & 4.13 & 4.19 & 0.82 & 0.39 & 0.29 & 0.56 & 0.72 & 0.51 & 1.32 \\
\hline NiO & - & - & 0.20 & - & - & - & 0.23 & - & - & - \\
\hline $\mathrm{ZnO}$ & 0.34 & 0.51 & 0.56 & 0.53 & 1.07 & 0.76 & 0.44 & 0.57 & 0.39 & 1.03 \\
\hline SrO & 0.25 & 1.13 & 1.14 & 0.11 & 0.06 & - & - & 0.05 & - & 0.19 \\
\hline $\mathrm{BaO}$ & 0.98 & 1.33 & 1.14 & - & 0.18 & - & - & - & - & 0.48 \\
\hline $\mathrm{PbO}$ & 1.23 & 0.61 & 1.39 & 0.30 & - & - & - & - & - & 0.51 \\
\hline $\mathrm{Na}_{2} \mathrm{O}$ & 0.07 & 0.30 & 0.22 & 0.07 & 0.08 & - & - & - & - & 0.32 \\
\hline $\mathrm{K}_{2} \mathrm{O}$ & 0.17 & 0.16 & 0.28 & 0.08 & 0.06 & - & - & 0.09 & 0.05 & 0.10 \\
\hline $\mathrm{P}_{2} \mathrm{O}_{5}$ & 0.13 & - & & - & 0.12 & - & - & - & - & - \\
\hline $\mathrm{La}_{2} \mathrm{O}_{3}$ & 0.11 & 0.53 & 0.56 & $\mathrm{n} / \mathrm{a}$ & n/a & $\mathrm{n} / \mathrm{a}$ & $n / a$ & n/a & $n / a$ & $n / a$ \\
\hline & 0.04 & & & - & - & - & - & 0.04 & - & 0.04 \\
\hline Total & 81.29 & 85.87 & 86.52 & 76.65 & 77.68 & 76.48 & 77.34 & 75.53 & 82.03 & 85.88 \\
\hline Si & 0.13 & 0.10 & 0.15 & 0.31 & 0.40 & 0.33 & 1.08 & 0.61 & 1.12 & 0.93 \\
\hline $\mathrm{Ti}$ & 0.06 & 0.09 & 0.09 & 0.15 & 0.13 & 0.03 & 0.07 & 0.10 & 0.14 & 0.11 \\
\hline $\mathrm{Mn}$ & 3.40 & 3.60 & 3.49 & 10.84 & 10.43 & 10.41 & 9.90 & 10.96 & 9.93 & 10.39 \\
\hline $\mathrm{Al}$ & 0.67 & 0.24 & 0.33 & 7.04 & 7.55 & 8.53 & 7.98 & 6.93 & 7.78 & 6.76 \\
\hline $\mathrm{Fe}$ & 0.12 & 0.30 & 0.27 & 0.69 & 0.59 & 0.08 & 0.23 & 0.42 & 0.35 & 0.54 \\
\hline $\mathrm{Mg}$ & 0.09 & 0.08 & 0.07 & 0.32 & 0.42 & 0.29 & 0.26 & 0.23 & 0.20 & 0.35 \\
\hline $\mathrm{Ca}$ & 0.42 & 0.35 & 0.35 & 0.27 & 0.13 & 0.09 & 0.18 & 0.24 & 0.15 & 0.39 \\
\hline $\mathrm{Ni}$ & & & 0.01 & - & - & - & 0.06 & - & - & - \\
\hline $\mathrm{Zn}$ & 0.02 & 0.03 & 0.03 & 0.12 & 0.24 & 0.17 & 0.10 & 0.13 & 0.08 & 0.21 \\
\hline $\mathrm{Sr}$ & 0.01 & 0.05 & 0.05 & 0.02 & 0.01 & - & - & 0.01 & - & 0.03 \\
\hline $\mathrm{Ba}$ & 0.03 & 0.04 & 0.03 & - & 0.02 & - & - & - & - & 0.05 \\
\hline $\mathrm{Pb}$ & 0.03 & 0.01 & 0.03 & 0.03 & - & - & - & - & - & 0.04 \\
\hline $\mathrm{Na}$ & 0.01 & 0.05 & 0.03 & 0.04 & 0.05 & - & - & - & - & 0.17 \\
\hline $\mathrm{K}$ & 0.02 & 0.02 & 0.03 & 0.03 & 0.02 & - & - & 0.04 & 0.02 & 0.04 \\
\hline La & 0.00 & 0.02 & 0.02 & $\mathrm{n} / \mathrm{a}$ & n/a & $\mathrm{n} / \mathrm{a}$ & $n / a$ & $\mathrm{n} / \mathrm{a}$ & $\mathrm{n} / \mathrm{a}$ & $\mathrm{n} / \mathrm{a}$ \\
\hline Total & 5.01 & 4.97 & 4.99 & 19.87 & 20.00 & 19.93 & 19.85 & 19.67 & 19.76 & 20.02 \\
\hline $\mathrm{O}^{-2}$ & 9.00 & 9.00 & 9.00 & 35.00 & 35.00 & 35.00 & 35.00 & 35.00 & 35.00 & 35.00 \\
\hline
\end{tabular}


FRACTURE-LINING ZEOLITES IN THE CALICO HILLS FORMATION AND IN THE CRATER FLAT GROUP (DIAGENETIC ZONES II AND III)

Analyses were accepted if totals were $>85 \%$ and $A \mid / \Sigma$ cations was between 0.95 and 1.05 .

For sample USW G-2 1761 and analcime in USW G-2 3137, analyses were accepted if $\mathrm{Al} / \Sigma$ cations was $\leq 1.10$ because there were no analyses below a ratio of 1.05 .

High Al/ cation ratios are attributed to Na loss. In sample USW G-2 1761, Na loss occurred because the grains are small, precluding movement of the sample during analysis or use of a large defocused beam. $\mathrm{Na}$ is the dominant cation in chabazite, so even a small $\mathrm{Na}$ loss was reflected in the $\mathrm{Al} / \Sigma$ cation ratio.

Sample numbers indicate drill hole and depth in feet.

$$
\begin{aligned}
\mathrm{n} / \mathrm{a} & =\text { not analyzed } \\
- & =\text { not detected }
\end{aligned}
$$


Clinoptilolite in Diagenetic Zone II

USW G-4 1436

\begin{tabular}{|c|c|c|c|c|c|c|c|c|c|c|c|c|c|c|c|c|}
\hline $\begin{array}{l}\mathrm{SiO}_{2} \\
\mathrm{Al}_{2} \mathrm{O}_{3}\end{array}$ & $\begin{array}{l}67.80 \\
11.95\end{array}$ & $\begin{array}{l}68.69 \\
12.38\end{array}$ & $\begin{array}{l}67.96 \\
12.07\end{array}$ & $\begin{array}{l}69.31 \\
12.14\end{array}$ & $\begin{array}{l}70.91 \\
12.05\end{array}$ & $\begin{array}{l}68.26 \\
11.97\end{array}$ & $\begin{array}{l}69.19 \\
12.23\end{array}$ & $\begin{array}{l}69.67 \\
12.32\end{array}$ & $\begin{array}{l}71.25 \\
12.38\end{array}$ & $\begin{array}{l}69.53 \\
12.27\end{array}$ & $\begin{array}{l}69.91 \\
12.45\end{array}$ & $\begin{array}{l}69.74 \\
12.37\end{array}$ & $\begin{array}{l}70.30 \\
12.56\end{array}$ & $\begin{array}{l}71.05 \\
12.50\end{array}$ & $\begin{array}{l}70.09 \\
12.44\end{array}$ & $\begin{array}{l}70.83 \\
12.56\end{array}$ \\
\hline $\mathrm{Fe}_{2} \mathrm{O}_{3}$ & - & - & - & - & - & - & - & - & - & - & - & - & - & - & - & \\
\hline $\mathrm{MgO}$ & - & - & - & - & - & - & - & - & - & - & - & - & - & - & - & \\
\hline $\mathrm{CaO}$ & 1.10 & 1.34 & 1.20 & 1.03 & 0.86 & 0.95 & 1.26 & 1.03 & 1.07 & 1.01 & 1.25 & 1.06 & 1.02 & 1.02 & 1.04 & 1.09 \\
\hline SrO & - & - & - & - & - & - & - & - & - & - & - & - & - & - & - & \\
\hline $\mathrm{BaO}$ & - & - & - & - & - & - & - & - & 0.09 & - & - & - & - & - & - & \\
\hline $\mathrm{Na}_{2} \mathrm{O}$ & 2.01 & 2.22 & 2.19 & 2.33 & 2.35 & 2.07 & 2.31 & 2.36 & 1.86 & 2.14 & 2.35 & 2.32 & 2.30 & 2.26 & 2.52 & 2.18 \\
\hline $\mathrm{K}_{2} \mathrm{O}$ & 5.70 & 5.31 & 5.83 & 6.31 & 6.46 & 6.13 & 5.49 & 5.69 & 7.01 & 6.55 & 5.79 & 6.01 & 6.03 & 6.65 & 6.17 & 5.91 \\
\hline Total & 88.56 & 89.94 & 89.25 & 91.12 & 92.63 & 89.38 & 90.48 & 91.07 & 93.66 & 91.50 & 91.75 & 91.50 & 92.21 & 93.48 & 92.26 & 92.57 \\
\hline
\end{tabular}

g

\begin{tabular}{|c|c|c|c|c|c|c|c|c|c|c|c|c|c|c|c|c|}
\hline $\begin{array}{l}\text { Si } \\
\text { Al }\end{array}$ & $\begin{array}{r}29.86 \\
6.20\end{array}$ & $\begin{array}{r}29.75 \\
6.32\end{array}$ & $\begin{array}{r}29.77 \\
6.23\end{array}$ & $\begin{array}{r}29.80 \\
6.15\end{array}$ & $\begin{array}{r}29.95 \\
6.00\end{array}$ & $\begin{array}{r}29.86 \\
6.17\end{array}$ & $\begin{array}{r}29.81 \\
6.21\end{array}$ & $\begin{array}{r}29.83 \\
6.22\end{array}$ & $\begin{array}{r}29.85 \\
6.11\end{array}$ & $\begin{array}{r}29.78 \\
6.19\end{array}$ & $\begin{array}{r}29.76 \\
6.25\end{array}$ & $\begin{array}{r}29.79 \\
6.23\end{array}$ & $\begin{array}{r}29.78 \\
6.27\end{array}$ & $\begin{array}{r}29.79 \\
6.18\end{array}$ & $\begin{array}{r}29.74 \\
6.22\end{array}$ & $\begin{array}{r}29.84 \\
6.24\end{array}$ \\
\hline $\mathrm{Mg}$ & - & - & - & - & - & - & - & & - & 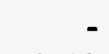 & - & - & 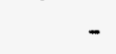 & 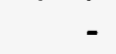 & - & \\
\hline $\mathrm{Ca}$ & 0.52 & 0.62 & 0.56 & 0.47 & 0.39 & 0.45 & 0.58 & 0.47 & 0.48 & 0.46 & 0.57 & 0.49 & 0.46 & 0.46 & 0.47 & 0.49 \\
\hline $\mathrm{Na}$ & 1.72 & 1.86 & 1.86 & 1.94 & 1.92 & 1.76 & 1.93 & 1.96 & 1.51 & 1.78 & 1.94 & 1.92 & 1.89 & 1.84 & 2.07 & 1.78 \\
\hline $\mathrm{K}$ & 3.20 & 2.93 & 3.26 & 3.46 & 3.48 & 3.42 & 3.02 & 3.11 & 3.75 & 3.58 & 3.14 & 3.27 & 3.26 & 3.56 & 3.34 & 3.18 \\
\hline Total & 41.50 & 41.49 & 41.68 & 41.83 & 41.75 & 41.65 & 41.55 & 41.59 & 41.72 & 41.80 & 41.66 & 41.70 & 41.66 & 41.82 & 41.85 & 41.52 \\
\hline $\mathrm{O}^{-2}$ & 72.00 & 72.00 & 72.00 & 72.00 & 72.00 & 72.00 & 72.00 & 72.00 & 72.00 & 72.00 & 72.00 & 72.00 & 72.00 & 72.00 & 72.00 & 72.00 \\
\hline$\Sigma$ cations & 5.96 & 6.04 & 6.24 & 6.35 & 6.18 & 6.07 & 6.11 & 6.01 & 6.25 & 6.28 & 6.22 & 6.17 & 6.07 & 6.31 & 6.36 & 5.94 \\
\hline $\mathrm{Al} / \Sigma$ cations & 1.04 & 1.05 & 1.00 & 0.97 & 0.97 & 1.02 & 1.02 & 1.03 & 0.98 & 0.99 & 1.00 & 1.01 & 1.03 & 0.98 & 0.98 & 1.05 \\
\hline Si/Al & 4.81 & 4.71 & 4.78 & 4.84 & 4.99 & 4.84 & 4.80 & 4.80 & 4.88 & 4.81 & 4.76 & 4.78 & 4.75 & 4.82 & 4.78 & 4.78 \\
\hline
\end{tabular}


Clinoptilolite in Diagenetic Zone II, continued

\begin{tabular}{|c|c|c|c|c|c|c|c|c|c|c|c|c|c|c|}
\hline & \multicolumn{2}{|c|}{ USW G-4 1436} & \multicolumn{3}{|c|}{ USW G-2 1761} & \multicolumn{9}{|c|}{ USW G-4 2698} \\
\hline & 70.76 & 70.67 & 69.69 & 69.64 & 69.33 & 70.16 & 72.21 & 70.74 & 69.96 & 71.47 & 69.65 & 69.67 & 69.97 & 70.96 \\
\hline $\begin{array}{l}\mathrm{Al}_{2} \mathrm{O}_{3} \\
\mathrm{Fe}_{2} \mathrm{O}_{3}\end{array}$ & $\begin{array}{r}12.66 \\
-\end{array}$ & $\begin{array}{r}12.36 \\
-\end{array}$ & $\begin{array}{r}12.43 \\
0.12\end{array}$ & $\begin{array}{r}12.51 \\
-\end{array}$ & $\begin{array}{r}12.85 \\
0.06\end{array}$ & $\begin{array}{r}13.25 \\
-\end{array}$ & $\begin{array}{r}12.92 \\
-\end{array}$ & $\begin{array}{r}12.77 \\
-\end{array}$ & $\begin{array}{r}13.27 \\
-\end{array}$ & $\begin{array}{r}12.59 \\
\end{array}$ & $\begin{array}{r}12.88 \\
-\end{array}$ & $\begin{array}{r}13.25 \\
-\end{array}$ & $\begin{array}{r}13.51 \\
-\end{array}$ & $\begin{array}{r}12.26 \\
-\end{array}$ \\
\hline $\mathrm{MgO}$ & - & - & 0.08 & 0.07 & 0.08 & 0.20 & 0.17 & 0.16 & 0.17 & 0.17 & 0.18 & 0.16 & 0.17 & 0.16 \\
\hline $\mathrm{CaO}$ & 1.22 & 1.28 & 2.95 & 2.84 & 3.07 & 4.78 & 4.37 & 4.43 & 4.67 & 4.23 & 4.45 & 4.72 & 4.72 & 4.23 \\
\hline SrO & - & - & - & 0.07 & - & - & - & - & - & - & - & - & - & - \\
\hline $\mathrm{BaO}$ & - & - & 0.10 & 0.11 & - & - & - & - & - & - & - & - & - & - \\
\hline $\mathrm{Na}_{2} \mathrm{O}$ & 2.49 & 2.26 & 0.94 & 1.06 & 0.99 & 1.50 & 1.55 & 1.48 & 1.50 & 1.43 & 1.54 & 1.60 & 1.71 & 1.49 \\
\hline $\mathrm{K}_{2} \mathrm{O}$ & 5.59 & 5.79 & 3.87 & 4.19 & 3.90 & 1.13 & 1.27 & 1.13 & 1.16 & 1.36 & 1.20 & 0.96 & 1.08 & 1.06 \\
\hline Total & 92.72 & 92.36 & 90.18 & 90.49 & 90.28 & 91.02 & 92.49 & 90.71 & 90.73 & 91.25 & 89.90 & 90.36 & 91.16 & 90.16 \\
\hline Si & 29.76 & 29.85 & 29.83 & 29.79 & 29.66 & 29.48 & 29.79 & 29.75 & 29.49 & 29.88 & 29.61 & 29.47 & 29.38 & 29.97 \\
\hline $\mathrm{Al}$ & 6.28 & 6.15 & 6.27 & 6.31 & 6.48 & 6.56 & 6.28 & 6.33 & 6.59 & 6.20 & 6.45 & 6.61 & 6.69 & 6.10 \\
\hline $\mathrm{Fe}$ & - & - & 0.04 & & 0.02 & - & - & - & - & - & - & - & - & - \\
\hline $\mathrm{Mg}$ & - & - & 0.05 & 0.04 & 0.05 & 0.13 & 0.10 & 0.10 & 0.11 & 0.11 & 0.11 & 0.10 & 0.11 & 0.10 \\
\hline $\mathrm{Ca}$ & 0.55 & 0.58 & 1.35 & 1.30 & 1.41 & 2.15 & 1.93 & 2.00 & 2.11 & 1.89 & 2.03 & 2.14 & 2.12 & 1.91 \\
\hline $\mathrm{Sr}$ & - & - & & 0.02 & - & - & - & - & - & - & - & - & - & - \\
\hline $\mathrm{Ba}$ & - & - & 0.02 & 0.02 & - & - & - & - & - & - & - & - & - & - \\
\hline $\mathrm{Na}$ & 2.03 & 1.85 & 0.78 & 0.88 & 0.82 & 1.22 & 1.24 & 1.21 & 1.23 & 1.16 & 1.27 & 1.31 & 1.39 & 1.22 \\
\hline K & 3.00 & 3.12 & 2.11 & 2.29 & 2.13 & 0.61 & 0.67 & 0.61 & 0.62 & 0.73 & 0.65 & 0.52 & 0.58 & 0.57 \\
\hline Total & 41.62 & 41.56 & 40.46 & 40.64 & 40.57 & 40.15 & 40.02 & 39.99 & 40.14 & 39.96 & 40.12 & 40.14 & 40.26 & 39.88 \\
\hline $\mathrm{O}^{-2}$ & 72.00 & 72.00 & 72.00 & 72.00 & 72.00 & 72.00 & 72.00 & 72.00 & 72.00 & 72.00 & 72.00 & 72.00 & 72.00 & 72.00 \\
\hline Ications & 6.13 & 6.13 & 5.74 & 5.93 & 5.87 & 6.38 & 5.98 & 6.01 & 6.28 & 5.89 & 6.20 & 6.31 & 6.43 & 5.82 \\
\hline Al/Lcations & 1.02 & 1.00 & 1.09 & 1.06 & 1.10 & 1.03 & 1.05 & 1.05 & 1.05 & 1.05 & 1.04 & 1.05 & 1.04 & 1.05 \\
\hline Si/Al & 4.74 & 4.85 & 4.76 & 4.72 & 4.58 & 4.49 & 4.74 & 4.70 & 4.47 & 4.82 & 4.59 & 4.46 & 4.39 & 4.91 \\
\hline
\end{tabular}


Chabazite in Diagenetic Zone II

UE-25b\#1 2165

\begin{tabular}{lrrrrrr} 
& & & & & & \\
\cline { 2 - 6 } $\mathrm{SiO}_{2}$ & 62.94 & 64.29 & 64.85 & 63.44 & 64.52 & 64.88 \\
$\mathrm{Al}_{2} \mathrm{O}_{3}$ & 16.04 & 15.86 & 16.22 & 15.64 & 15.83 & 16.02 \\
$\mathrm{Fe}_{2} \mathrm{O}_{3}$ & - & - & - & - & - & - \\
$\mathrm{MgO}$ & - & - & - & - & - & - \\
$\mathrm{CaO}$ & 2.59 & 2.84 & 2.68 & 2.63 & 2.78 & 2.80 \\
$\mathrm{SrO}$ & - & - & - & - & - & - \\
$\mathrm{BaO}$ & - & - & - & - & - & - \\
$\mathrm{Na} \mathrm{O}_{2} \mathrm{O}$ & 3.78 & 3.62 & 2.99 & 4.35 & 3.87 & 3.80 \\
$\mathrm{~K} \mathrm{O}$ & 3.60 & 3.86 & 5.12 & 3.52 & 4.30 & 4.28 \\
$\mathrm{Total}$ & 88.95 & 90.47 & 91.86 & 89.58 & 91.30 & 91.78 \\
& & & & & & \\
$\mathrm{Si}$ & & & & & & \\
$\mathrm{Al}$ & 27.81 & 27.95 & 27.89 & 27.88 & 27.90 & 27.89 \\
$\mathrm{Fe}$ & 8.35 & 8.13 & 8.22 & 8.10 & 8.07 & 8.12 \\
$\mathrm{Ca}$ & - & - & - & - & - & - \\
$\mathrm{Na}$ & 1.23 & 1.32 & 1.23 & 1.24 & 1.29 & 1.29 \\
$\mathrm{~K}$ & 3.24 & 3.05 & 2.49 & 3.71 & 3.24 & 3.17 \\
$\mathrm{Total}$ & 2.03 & 2.14 & 2.81 & 1.97 & 2.37 & 2.35 \\
$\mathrm{O}^{-2}$ & 42.65 & 42.59 & 42.65 & 42.91 & 42.87 & 42.81 \\
$\Sigma$ cations & 72.00 & 72.00 & 72.00 & 72.00 & 72.00 & 72.00 \\
& 7.72 & 7.84 & 7.77 & 8.16 & 8.19 & 8.09 \\
$\mathrm{Al} / \Sigma$ cations & 1.08 & 1.04 & 1.06 & 0.99 & 0.98 & 1.00 \\
$\mathrm{Si} / \mathrm{Al}$ & 3.33 & 3.44 & 3.39 & 3.44 & 3.46 & 3.44 \\
& & & & & & \\
\hline & & & & & &
\end{tabular}


Analcime in Diagenetic Zone III

USW G-2 3137

\begin{tabular}{|c|c|c|c|c|c|c|c|c|c|c|c|c|}
\hline $\begin{array}{l}\mathrm{SiO}_{2} \\
\mathrm{Al}_{2} \mathrm{O}_{3}\end{array}$ & $\begin{array}{l}60.29 \\
19.65\end{array}$ & $\begin{array}{l}60.77 \\
19.60\end{array}$ & $\begin{array}{l}60.39 \\
19.83\end{array}$ & $\begin{array}{l}60.93 \\
19.85\end{array}$ & $\begin{array}{l}59.66 \\
20.38\end{array}$ & $\begin{array}{l}60.48 \\
19.70\end{array}$ & $\begin{array}{l}60.05 \\
19.95\end{array}$ & $\begin{array}{l}59.58 \\
19.77\end{array}$ & $\begin{array}{l}59.79 \\
20.09\end{array}$ & $\begin{array}{l}59.45 \\
20.15\end{array}$ & $\begin{array}{l}60.79 \\
19.56\end{array}$ & $\begin{array}{l}60.48 \\
20.07\end{array}$ \\
\hline $\mathrm{Fe}_{2} \mathrm{O}_{3}$ & - & - & - & - & - & - & - & - & - & - & - & - \\
\hline $\mathrm{MgO}$ & - & - & - & - & - & - & - & - & - & - & - & - \\
\hline $\mathrm{CaO}$ & - & - & - & - & - & - & - & - & - & - & - & - \\
\hline SrO & - & - & - & - & - & - & - & - & - & - & - & - \\
\hline $\mathrm{BaO}$ & - & - & - & - & - & - & - & - & - & - & - & - \\
\hline $\mathrm{Na}_{2} \mathrm{O}$ & 11.39 & 11.21 & 11.49 & 11.33 & 11.68 & 11.30 & 11.38 & 11.43 & 11.49 & 11.34 & 11.16 & 11.50 \\
\hline $\mathrm{K}_{2} \mathrm{O}$ & - & - & - & - & - & - & - & - & - & - & - & - \\
\hline Total & 91.33 & 91.58 & 91.71 & 92.11 & 91.72 & 91.48 & 91.38 & 90.78 & 91.37 & 90.94 & 91.51 & 92.05 \\
\hline $\mathrm{Si}$ & 2.17 & 2.18 & 2.17 & 2.18 & 2.15 & 2.18 & 2.17 & 2.16 & 2.16 & 2.16 & 2.18 & 2.17 \\
\hline $\mathrm{Al}$ & 0.84 & 0.83 & 0.84 & 0.84 & 0.86 & 0.84 & 0.85 & 0.85 & 0.85 & 0.86 & 0.83 & 0.85 \\
\hline $\mathrm{Na}$ & 0.80 & 0.78 & 0.80 & 0.78 & 0.82 & 0.79 & 0.80 & 0.80 & 0.80 & 0.80 & 0.78 & 0.80 \\
\hline Total & 3.81 & 3.79 & 3.81 & 3.80 & 3.83 & 3.80 & 3.81 & 3.82 & 3.82 & 3.81 & 3.79 & 3.81 \\
\hline $\mathrm{O}^{-2}$ & 6.00 & 6.00 & 6.00 & 6.00 & 6.00 & 6.00 & 6.00 & 6.00 & 6.00 & 6.00 & 6.00 & 6.00 \\
\hline $\mathrm{Al} / \Sigma$ cations & 1.05 & 1.06 & 1.05 & 1.06 & 1.06 & 1.06 & 1.07 & 1.05 & 1.06 & 1.08 & 1.07 & 1.06 \\
\hline Si/Al & 2.60 & 2.63 & 2.58 & 2.60 & 2.48 & 2.60 & 2.55 & 2.56 & 2.53 & 2.50 & 2.64 & 2.56 \\
\hline
\end{tabular}




\section{APPENDIX V}

FRACTURE-LINING MANGANESE-OXIDE MINERALS IN THE CRATER FLAT GROUP

All analyses were previously published in LA-11787-MS (Carlos et al., 1990) and are included in this appendix for completeness. Mineral identifications are given where known. Many samples contained finely intergrown crystals of two manganese-oxide minerals or of hematite and cryptomelane-hollandite. Analyses, although representative of the coating present, may not be of single phases.

Sample numbers indicate drill hole and depth in feet.

$$
\begin{aligned}
n / a & =\text { not analyzed } \\
-\quad & =\text { not detected } \\
* & =\text { possible presence of }<0.5 \% \mathrm{CeO}_{2} \\
* * & =\text { possible presence of }>0.5 \% \mathrm{CeO}_{2}
\end{aligned}
$$


Manganese Oxides from USW G-4 1990-1991 ${ }^{\text {a }}$

Pyrolusite

\begin{tabular}{|c|c|c|c|c|c|c|c|c|c|c|c|}
\hline \multirow[b]{2}{*}{$\mathrm{SiO}_{2}$} & \multicolumn{3}{|c|}{ Pyrolusite } & \multicolumn{8}{|c|}{ Cryptomelane-Hollandite } \\
\hline & 1.82 & 2.62 & 1.69 & 0.25 & 0.44 & 0.24 & 0.26 & 0.58 & 0.20 & 0.56 & 0.16 \\
\hline $\mathrm{TiO}_{2}$ & - & - & 0.22 & - & 0.11 & - & - & - & - & - & - \\
\hline $\mathrm{MnO}_{2}$ & 86.56 & 85.42 & 91.78 & 88.60 & 86.64 & 87.13 & 83.43 & 82.02 & 85.66 & 83.85 & 86.67 \\
\hline $\mathrm{CeO}_{2}$ & 0.15 & 0.14 & 0.16 & - & - & - & $\mathrm{n} / \mathrm{a}$ & - & $\mathrm{n} / \mathrm{a}$ & $\mathrm{n} / \mathrm{a}$ & - \\
\hline $\mathrm{Al}_{2} \mathrm{O}_{3}$ & 2.72 & 2.80 & 1.87 & 0.45 & 0.44 & 0.46 & 0.78 & 0.57 & 0.48 & 0.75 & 0.54 \\
\hline $\mathrm{Fe}_{2} \mathrm{O}_{3}$ & 0.21 & 0.17 & 0.60 & 0.89 & 0.69 & 0.83 & 0.74 & 0.72 & 0.73 & 1.31 & 0.69 \\
\hline $\mathrm{MgO}$ & - & - & - & - & - & - & - & - & - & - & - \\
\hline $\mathrm{CaO}$ & & 0.13 & 0.11 & 0.09 & 0.09 & 0.11 & 0.22 & 0.25 & 0.24 & 0.23 & 0.23 \\
\hline SrO & & - & - & - & - & 0.19 & 0.09 & 0.12 & 0.07 & - & 0.07 \\
\hline $\mathrm{BaO}$ & 0.11 & - & - & 4.89 & 5.02 & 5.05 & 5.61 & 5.74 & 5.84 & 5.95 & 6.03 \\
\hline $\mathrm{Na}_{2} \mathrm{O}$ & - & - & 0.11 & 0.31 & 0.29 & 0.23 & 0.36 & 0.35 & 0.31 & 0.36 & 0.37 \\
\hline $\mathrm{K}_{2} \mathrm{O}$ & 0.05 & 0.05 & 0.06 & 4.74 & 4.83 & 4.65 & 3.34 & 3.19 & 3.88 & 3.86 & 4.13 \\
\hline Total & 91.62 & 91.33 & 96.60 & 100.22 & 98.55 & 98.89 & 94.83 & 93.54 & 97.41 & 96.87 & 98.89 \\
\hline $\mathrm{Si}^{+4}$ & 0.23 & 0.33 & 0.20 & 0.03 & 0.06 & 0.03 & 0.03 & 0.08 & 0.03 & 0.07 & 0.02 \\
\hline $\mathrm{Ti}^{+4}$ & - & - & 0.02 & - & 0.01 & - & - & - & - & - & - \\
\hline $\mathrm{Mn}^{+4}$ & 7.44 & 7.34 & 7.52 & 7.53 & 7.50 & 7.52 & 7.50 & 7.48 & 7.53 & 7.40 & 7.52 \\
\hline $\mathrm{Ce}^{+4}$ & 0.01 & 0.01 & 0.01 & - & - & - & $n / a$ & - & $\mathrm{n} / \mathrm{a}$ & $\mathrm{n} / \mathrm{a}$ & - \\
\hline $\mathrm{Al}^{+3}$ & 0.40 & 0.41 & 0.26 & 0.07 & 0.06 & 0.07 & 0.12 & 0.09 & 0.07 & 0.11 & 0.08 \\
\hline $\mathrm{Fe}^{+3}$ & 0.02 & 0.02 & 0.05 & 0.08 & 0.07 & 0.08 & 0.07 & 0.07 & 0.07 & 0.13 & 0.07 \\
\hline $\mathrm{Ca}^{+2}$ & 0.01 & 0.02 & 0.01 & 0.01 & 0.01 & 0.01 & 0.03 & 0.04 & 0.03 & 0.03 & 0.03 \\
\hline $\mathrm{Sr}^{+2}$ & - & - & - & - & - & 0.01 & 0.01 & 0.01 & 0.01 & - & 0.01 \\
\hline $\mathrm{Ba}^{+2}$ & - & - & - & 0.24 & 0.25 & 0.25 & 0.29 & 0.30 & 0.29 & 0.30 & 0.30 \\
\hline $\mathrm{Na}^{+1}$ & - & - & 0.03 & 0.07 & 0.07 & 0.06 & 0.09 & 0.09 & 0.08 & 0.09 & 0.09 \\
\hline $\mathrm{K}^{+1}$ & 0.01 & 0.01 & 0.01 & 0.74 & 0.77 & 0.74 & 0.55 & 0.54 & 0.63 & 0.63 & 0.66 \\
\hline Total & 8.12 & 8.12 & 8.11 & 8.77 & 8.79 & 8.77 & 8.69 & 8.68 & 8.73 & 8.76 & 8.77 \\
\hline $\mathrm{O}^{-2}$ & 16.00 & 16.00 & 16.00 & 16.00 & 16.00 & 16.00 & 16.00 & 16.00 & 16.00 & 16.00 & 16.00 \\
\hline
\end{tabular}

Cryptomelane-Hollandite 
Manganese Oxides from USW G-4 1990-1991, continued

Cryptomelane-Hollandite

$\begin{array}{lcccccccc}\mathrm{SiO}_{2} & 0.68 & 0.24 & 0.26 & 0.19 & 0.27 & 0.55 & 0.29 & 0.26 \\ \mathrm{TiO}_{2} & - & - & - & - & - & - & 0.11 & - \\ \mathrm{MnO}_{2} & 82.52 & 85.90 & 83.23 & 86.94 & 83.83 & 84.12 & 83.68 & 84.54 \\ \mathrm{CeO}_{2} & \mathrm{n} / \mathrm{a} & n / a & n / a & n / a & n / a & - & n / a & - \\ \mathrm{Al}_{2} \mathrm{O}_{3} & 0.56 & 0.62 & 0.52 & 0.62 & 0.43 & 0.34 & 0.53 & 0.42 \\ \mathrm{Fe}_{2} \mathrm{O}_{3} & 0.83 & 0.94 & 0.65 & 0.52 & 0.91 & 1.03 & 0.65 & 0.61 \\ \mathrm{MgO} & - & - & - & - & - & - & - & - \\ \mathrm{CaO} & 0.24 & 0.24 & 0.23 & 0.19 & 0.21 & 0.14 & 0.26 & 0.22 \\ \mathrm{SrO} & 0.10 & 0.07 & 0.07 & 0.07 & 0.06 & - & 0.05 & 0.07 \\ \mathrm{BaO} & 6.09 & 6.31 & 6.31 & 6.55 & 8.32 & 8.62 & 9.02 & 9.23 \\ \mathrm{Na} 2 & 0.40 & 0.41 & 0.36 & 0.40 & 0.37 & 0.33 & 0.37 & 0.32 \\ \mathrm{~K}_{2} \mathrm{O} & 3.23 & 3.63 & 3.57 & 3.61 & 2.52 & 2.91 & 2.34 & 2.13 \\ \mathrm{Total}^{2} & 94.65 & 98.36 & 95.20 & 99.09 & 96.92 & 98.04 & 97.30 & 97.80 \\ & & & & & & & & \\ \mathrm{Si}^{+4} & 0.09 & 0.03 & 0.03 & 0.02 & 0.03 & 0.07 & 0.04 & 0.03 \\ \mathrm{Ti}^{+4} & - & - & - & - & - & - & 0.01 & - \\ \mathrm{Mn}^{+4} & 7.45 & 7.49 & 7.51 & 7.53 & 7.49 & 7.45 & 7.48 & 7.52 \\ \mathrm{Ce}^{+4} & n / a & n / a & n / a & n / a & n / a & - & n / a & - \\ \mathrm{Al}^{+3} & 0.09 & 0.09 & 0.08 & 0.09 & 0.07 & 0.05 & 0.08 & 0.06 \\ \mathrm{Fe}^{+3} & 0.08 & 0.09 & 0.06 & 0.05 & 0.09 & 0.10 & 0.06 & 0.06 \\ \mathrm{Ca}^{+2} & 0.03 & 0.03 & 0.03 & 0.03 & 0.03 & 0.02 & 0.04 & 0.03 \\ \mathrm{Sr}^{+2} & 0.01 & 0.01 & 0.01 & 0.01 & 0.00 & - & 0.00 & 0.01 \\ \mathrm{Ba}^{+2} & 0.31 & 0.31 & 0.32 & 0.32 & 0.42 & 0.43 & 0.46 & 0.47 \\ \mathrm{Na}^{+1} & 0.10 & 0.10 & 0.09 & 0.10 & 0.09 & 0.08 & 0.09 & 0.08 \\ \mathrm{~K}^{+1} & 0.54 & 0.58 & 0.59 & 0.58 & 0.42 & 0.48 & 0.39 & 0.35 \\ \mathrm{Total}^{+1} & 8.70 & 8.73 & 8.73 & 8.72 & 8.65 & 8.68 & 8.64 & 8.60 \\ \mathrm{O}^{-2} & 16.00 & 16.00 & 16.00 & 16.00 & 16.00 & 16.00 & 16.00 & 16.00 \\ & & & & & & & & \end{array}$

${ }^{a}$ Analyzed for but not detected: $\mathrm{Co}, \mathrm{Ni}, \mathrm{Cu}, \mathrm{Zn}, \mathrm{Pb}, \mathrm{P}, \mathrm{La}, \mathrm{Y}$, and $\mathrm{Cl}$ 
Manganese and Iron Oxides from USW G-4 2099-2100

\begin{tabular}{|c|c|c|c|c|c|c|c|c|c|}
\hline \multirow[b]{2}{*}{$\mathrm{SiO}_{2}$} & \multicolumn{4}{|c|}{ Iron Oxides } & \multicolumn{5}{|c|}{ Pyrolusite } \\
\hline & 9.01 & 8.37 & 8.95 & 5.52 & 1.61 & 0.71 & 0.83 & 0.85 & 0.77 \\
\hline $\mathrm{TiO}_{2}$ & - & - & - & - & - & 0.24 & - & - & - \\
\hline $\mathrm{MnO}_{2}$ & 2.02 & 2.29 & 2.31 & 1.93 & 86.27 & 92.32 & 91.07 & 90.14 & 93.90 \\
\hline $\mathrm{CeO}_{2}$ & 0.10 & - & - & - & 0.12 & 0.20 & 0.21 & 0.26 & - \\
\hline $\mathrm{Al}_{2} \mathrm{O}_{3}$ & 0.87 & 0.85 & 0.95 & 0.62 & 1.36 & 0.94 & 1.49 & 1.08 & 1.19 \\
\hline $\mathrm{Fe}_{2} \mathrm{O}_{3}$ & 73.60 & 73.33 & 74.03 & 84.10 & 2.98 & 2.15 & 3.00 & 1.17 & 2.24 \\
\hline $\mathrm{MgO}$ & - & - & 0.05 & - & - & - & - & - & - \\
\hline $\mathrm{CaO}$ & 0.16 & 0.09 & 0.11 & 0.25 & 0.07 & 0.07 & 0.07 & 0.05 & 0.09 \\
\hline $\mathrm{NiO}$ & 0.12 & - & - & - & - & - & 0.12 & - & - \\
\hline $\mathrm{ZnO}$ & - & - & - & - & 0.21 & - & - & 0.16 & - \\
\hline SrO & - & - & - & - & - & - & - & - & - \\
\hline $\mathrm{BaO}$ & - & - & 0.20 & - & - & 0.22 & 0.40 & 0.43 & 1.20 \\
\hline $\mathrm{PbO}$ & 0.30 & 0.39 & 0.27 & $n / a$ & - & - & - & - & 0.48 \\
\hline $\mathrm{Na}_{2} \mathrm{O}$ & - & - & - & - & - & - & - & - & - \\
\hline $\mathrm{K}_{2} \mathrm{O}$ & - & - & 0.07 & 0.06 & - & - & - & - & 0.10 \\
\hline Total & 86.18 & 85.32 & 86.94 & 92.48 & 92.62 & 96.85 & 97.19 & 94.14 & 99.97 \\
\hline $\mathrm{Si}^{+4}$ & 1.36 & 1.28 & 1.34 & 0.80 & 0.20 & 0.08 & 0.10 & 0.10 & 0.09 \\
\hline $\mathrm{Ti}^{+4}$ & - & - & - & - & - & 0.02 & - & - & - \\
\hline $\mathrm{Mn}^{+4}$ & 0.21 & 0.24 & 0.24 & 0.19 & 7.42 & 7.63 & 7.51 & 7.66 & 7.59 \\
\hline $\mathrm{Ce}^{+4}$ & 0.01 & - & - & - & 0.01 & 0.01 & 0.01 & 0.01 & - \\
\hline $\mathrm{Al}^{+3}$ & 0.15 & 0.15 & 0.17 & 0.11 & 0.20 & 0.13 & 0.21 & 0.16 & 0.16 \\
\hline $\mathrm{Fe}^{+3}$ & 8.37 & 8.46 & 8.35 & 9.20 & 0.28 & 0.19 & 0.27 & 0.11 & 0.20 \\
\hline $\mathrm{Mg}^{+2}$ & - & - & 0.01 & - & - & - & - & - & - \\
\hline $\mathrm{Ca}^{+2}$ & 0.03 & 0.01 & 0.02 & 0.04 & 0.01 & 0.01 & 0.01 & 0.01 & 0.01 \\
\hline $\mathrm{Ni}^{+2}$ & 0.01 & - & - & - & - & - & 0.01 & - & - \\
\hline $\mathrm{Zn}^{+2}$ & - & - & - & - & 0.02 & - & - & 0.01 & - \\
\hline $\mathrm{Sr}^{+2}$ & - & - & - & - & - & - & - & - & - \\
\hline $\mathrm{Ba}^{+2}$ & - & - & 0.01 & - & - & 0.01 & 0.02 & 0.02 & 0.06 \\
\hline $\mathrm{Pb}^{+2}$ & 0.01 & 0.02 & 0.01 & $\mathrm{n} / \mathrm{a}$ & - & - & - & - & 0.02 \\
\hline $\mathrm{Na}^{+1}$ & . & - & - & - & - & - & - & - & - \\
\hline $\mathrm{K}^{+1}$ & - & - & 0.01 & 0.01 & - & - & - & - & 0.01 \\
\hline Total & 10.16 & 10.17 & 10.17 & 10.35 & 8.13 & 8.09 & 8.14 & 8.09 & 8.14 \\
\hline $\mathrm{O}^{-2}$ & 16.00 & 16.00 & 16.00 & 16.00 & 16.00 & 16.00 & 16.00 & 16.00 & 16.00 \\
\hline
\end{tabular}


Manganese and Iron Oxides from USW G-4 2099-2100, continued

Coronadite

\begin{tabular}{ccccc}
\hline & & & & \\
1.33 & 0.97 & 0.66 & 1.04 & 0.83 \\
- & - & - & - & - \\
70.83 & 72.49 & 74.16 & 72.72 & 74.09 \\
- & - & n/a & n/a & - \\
0.60 & 0.19 & 0.18 & 0.27 & 0.24 \\
1.58 & 1.19 & 1.10 & 1.54 & 1.15
\end{tabular}

$\mathrm{MgO}$

$\mathrm{CaO}$

$\mathrm{NiO}$

$\mathrm{ZnO}$

SrO

$\mathrm{BaO}$

$\mathrm{PbO}$

$\mathrm{Na}_{2} \mathrm{O}$

$\mathrm{K}_{2} \mathrm{O}$

Total

$\mathrm{Si}^{+4}$

$\mathrm{Ti}^{+4}$

$\mathrm{Ce}^{+4}$

$\mathrm{Al}^{+3}$

$\mathrm{Fe}^{+3}$

$\mathrm{Mg}^{+2}$

$\mathrm{Ca}^{+2}$

$\mathrm{Zn}^{+2}$

$\mathrm{Sr}^{+2}$

$\mathrm{Ba}^{+2}$

$\mathrm{Pb}^{+2}$

$\mathrm{Na}^{+1}$

$\mathrm{K}^{+1}$

Total

$\mathrm{O}^{-2}$
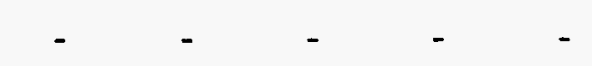
Manganese and Iron Oxides from USW G-4 2099-2100, continued

Hollandite

\begin{tabular}{|c|c|c|c|c|c|c|c|c|c|c|c|c|}
\hline $\mathrm{SiO}_{2}$ & 0.35 & 0.89 & 0.89 & 0.14 & 0.14 & 0.26 & 0.30 & 0.09 & 0.16 & 0.43 & 0.05 & 0.46 \\
\hline $\mathrm{TiO}_{2}$ & 0.13 & 0.10 & - & 0.08 & 0.07 & - & - & - & - & 0.15 & - & - \\
\hline $\mathrm{MnO}_{2}$ & 78.11 & 70.54 & 72.28 & 81.80 & 80.84 & 81.48 & 76.16 & 81.22 & 81.78 & 74.46 & 81.98 & 78.90 \\
\hline $\mathrm{CeO}_{2}$ & * & * & * & n/a & n/a & n/a & * & - & n/a & * & n/a & * \\
\hline $\mathrm{Al}_{2} \mathrm{O}_{3}$ & 0.42 & 0.78 & 0.63 & 0.44 & 0.39 & 0.32 & 0.49 & 0.42 & 0.49 & 0.53 & 0.35 & 0.46 \\
\hline $\mathrm{Fe}_{2} \mathrm{O}_{3}$ & 1.24 & 2.41 & 2.52 & 0.59 & 0.88 & 1.50 & 1.03 & 0.60 & 0.66 & 1.18 & 0.58 & 1.68 \\
\hline $\mathrm{MgO}$ & - & 0.06 & - & - & - & - & - & - & - & 0.30 & - & - \\
\hline $\mathrm{CaO}$ & 0.15 & 0.08 & 0.07 & 0.11 & 0.07 & - & 0.15 & 0.09 & 0.10 & 0.16 & 0.07 & 0.08 \\
\hline $\mathrm{NiO}$ & - & - & - & - & - & - & 0.14 & - & - & - & - & - \\
\hline $\mathrm{ZnO}$ & - & - & - & - & 0.11 & - & - & - & - & - & - & - \\
\hline SrO & - & - & - & - & - & - & - & - & - & - & - & - \\
\hline $\mathrm{BaO}$ & 12.07 & 12.19 & 12.29 & 12.50 & 12.58 & 12.79 & 12.80 & 12.94 & 13.02 & 13.04 & 13.05 & 13.11 \\
\hline $\mathrm{PbO}$ & $\mathrm{n} / \mathrm{a}$ & $\mathrm{n} / \mathrm{a}$ & $\mathrm{n} / \mathrm{a}$ & 0.47 & 0.57 & 0.29 & $n / a$ & 0.43 & 0.51 & $n / a$ & 0.33 & $n / a$ \\
\hline $\mathrm{Na}_{2} \mathrm{O}$ & 0.13 & 0.10 & - & 0.12 & 0.19 & - & 0.21 & 0.13 & 0.12 & 0.14 & 0.19 & 0.16 \\
\hline $\mathrm{K}_{2} \mathrm{O}$ & 1.22 & 0.79 & 0.75 & 1.11 & 1.02 & 0.55 & 1.08 & 1.14 & 0.83 & 1.12 & 1.03 & 0.62 \\
\hline Total & 93.82 & 87.94 & 89.43 & 97.36 & 96.86 & 97.19 & 92.36 & 97.06 & 97.67 & 91.51 & 97.63 & 95.47 \\
\hline $\mathrm{Si}^{+4}$ & 0.05 & 0.13 & 0.13 & 0.02 & 0.02 & 0.03 & 0.04 & 0.01 & 0.02 & 0.06 & 0.01 & 0.06 \\
\hline $\mathrm{Ti}^{+4}$ & 0.01 & 0.01 & - & 0.01 & 0.01 & - & - & - & - & 0.02 & - & - \\
\hline $\mathrm{Mn}^{+4}$ & 7.40 & 7.15 & 7.20 & 7.48 & 7.46 & 7.46 & 7.38 & 7.48 & 7.48 & 7.30 & 7.50 & 7.36 \\
\hline $\mathrm{Ce}^{+4}$ & - & - & - & $\mathrm{n} / \mathrm{a}$ & $\mathrm{n} / \mathrm{a}$ & $\mathrm{n} / \mathrm{a}$ & - & - & $\mathrm{n} / \mathrm{a}$ & - & $\mathrm{n} / \mathrm{a}$ & - \\
\hline $\mathrm{Al}^{+3}$ & 0.07 & 0.13 & 0.11 & 0.07 & 0.06 & 0.05 & 0.08 & 0.07 & 0.08 & 0.09 & 0.05 & 0.07 \\
\hline $\mathrm{Fe}^{+3}$ & 0.13 & 0.27 & 0.27 & 0.06 & 0.09 & 0.15 & 0.11 & 0.06 & 0.07 & 0.13 & 0.06 & 0.17 \\
\hline $\mathrm{Mg}^{+2}$ & - & 0.01 & - & - & - & - & - & - & - & 0.06 & - & - \\
\hline $\mathrm{Ca}^{+2}$ & 0.02 & 0.01 & 0.01 & 0.02 & 0.01 & - & 0.02 & 0.01 & 0.01 & 0.02 & 0.01 & 0.01 \\
\hline $\mathrm{Ni}^{+2}$ & - & - & - & - & - & - & 0.02 & - & - & - & - & - \\
\hline $\mathrm{Zn}^{+2}$ & - & - & - & - & 0.01 & - & - & - & - & - & - & - \\
\hline $\mathrm{Sr}^{+2}$ & - & - & - & - & - & - & - & - & - & - & - & - \\
\hline $\mathrm{Ba}^{+2}$ & 0.65 & 0.70 & 0.69 & 0.65 & 0.66 & 0.66 & 0.70 & 0.68 & 0.67 & 0.72 & 0.68 & 0.69 \\
\hline $\mathrm{Pb}^{+2}$ & n/a & $\mathrm{n} / \mathrm{a}$ & n/a & 0.02 & 0.02 & 0.01 & $n / a$ & 0.02 & 0.02 & $\mathrm{n} / \mathrm{a}$ & 0.01 & $\mathrm{n} / \mathrm{a}$ \\
\hline $\mathrm{Na}^{+1}$ & 0.03 & 0.03 & - & 0.03 & 0.05 & - & 0.06 & 0.03 & 0.03 & 0.04 & 0.05 & 0.04 \\
\hline $\mathrm{K}^{+1}$ & 0.21 & 0.15 & 0.14 & 0.19 & 0.17 & 0.09 & 0.19 & 0.19 & 0.14 & 0.20 & 0.17 & 0.11 \\
\hline Total & 8.57 & 8.60 & 8.55 & 8.54 & 8.55 & 8.46 & 8.61 & 8.55 & 8.52 & 8.64 & 8.54 & 8.53 \\
\hline $\mathrm{O}^{-2}$ & 16.00 & 16.00 & 16.00 & 16.00 & 16.00 & 16.00 & 16.00 & 16.00 & 16.00 & 16.00 & 16.00 & 16.00 \\
\hline
\end{tabular}


Hollandite

\begin{tabular}{|c|c|c|c|c|c|c|c|c|c|c|c|}
\hline $\mathrm{SiO}_{2}$ & 0.14 & 0.46 & 0.32 & 0.12 & 0.33 & 1.05 & 1.10 & 1.29 & 0.35 & 0.19 & 0.21 \\
\hline $\mathrm{TiO}_{2}$ & - & 0.13 & - & - & 0.16 & - & - & - & - & 0.13 & - \\
\hline $\mathrm{MnO}_{2}$ & 81.47 & 81.44 & 82.01 & 80.98 & 78.70 & 80.20 & 75.78 & 77.71 & 71.13 & 81.64 & 84.36 \\
\hline $\mathrm{CeO}_{2}$ & n/a & * & - & * & * & $\mathrm{n} / \mathrm{a}$ & - & $\star$ & - & - & - \\
\hline $\mathrm{Al}_{2} \mathrm{O}_{3}$ & 0.41 & 0.49 & 0.42 & 0.28 & 0.30 & 0.69 & 0.68 & 0.56 & 1.82 & 1.01 & 0.91 \\
\hline $\mathrm{Fe}_{2} \mathrm{O}_{3}$ & 0.62 & 1.76 & 1.47 & 0.77 & 1.92 & 4.24 & 3.56 & 3.75 & 0.47 & 0.33 & 0.28 \\
\hline $\mathrm{MgO}$ & - & - & - & 0.32 & 0.31 & - & - & - & 0.05 & 0.08 & 0.05 \\
\hline $\mathrm{CaO}$ & 0.11 & 0.07 & - & 0.12 & 0.06 & 0.10 & 0.08 & 0.16 & 0.09 & 0.13 & 0.11 \\
\hline $\mathrm{NiO}$ & - & - & - & - & 0.17 & - & - & - & - & - & - \\
\hline $\mathrm{ZnO}$ & - & - & - & - & - & - & - & - & 0.15 & 0.23 & 0.15 \\
\hline SrO & - & - & 0.05 & 0.20 & 0.19 & - & - & 0.23 & 0.05 & 0.11 & 0.13 \\
\hline $\mathrm{BaO}$ & 13.40 & 13.45 & 14.02 & 14.03 & 14.26 & 14.96 & 15.03 & 15.79 & 10.57 & 11.09 & 10.88 \\
\hline $\mathrm{PbO}$ & 0.57 & $\mathrm{n} / \mathrm{a}$ & 0.33 & n/a & $\mathrm{n} / \mathrm{a}$ & - & - & n/a & 0.26 & 0.20 & 0.22 \\
\hline $\mathrm{Na}_{2} \mathrm{O}$ & 0.14 & 0.10 & 0.13 & 0.16 & 0.13 & 0.17 & 0.21 & 0.18 & 0.28 & 0.14 & 0.17 \\
\hline $\mathrm{K}_{2} \mathrm{O}$ & 1.07 & 0.70 & 0.67 & 1.02 & 0.54 & 0.43 & 0.38 & 0.40 & 1.42 & 1.98 & 2.62 \\
\hline $\mathrm{La}_{2} \mathrm{O}_{3}$ & - & - & - & - & - & - & - & - & 0.13 & 0.17 & 0.14 \\
\hline $\mathrm{P}_{2} \mathrm{O}_{5}$ & n/a & $n / a$ & $n / a$ & $\mathrm{n} / \mathrm{a}$ & $\mathrm{n} / \mathrm{a}$ & $n / a$ & $\mathrm{n} / \mathrm{a}$ & $\mathrm{n} / \mathrm{a}$ & - & 0.05 & - \\
\hline Total & 97.93 & 98.60 & 99.42 & 98.00 & 97.07 & 101.84 & 96.82 & 100.07 & 86.77 & 97.48 & 100.23 \\
\hline $\mathrm{Si}^{+4}$ & 0.02 & 0.06 & 0.04 & 0.02 & 0.04 & 0.13 & 0.15 & 0.17 & 0.05 & 0.02 & 0.03 \\
\hline $\mathrm{Ti}^{+4}$ & - & 0.01 & - & - & 0.02 & - & - & - & - & 0.01 & - \\
\hline $\mathrm{Mn}^{+4}$ & 7.47 & 7.36 & 7.40 & 7.43 & 7.30 & 7.07 & 7.07 & 7.04 & 7.25 & 7.40 & 7.43 \\
\hline $\mathrm{Ce}^{+4}$ & $\mathrm{n} / \mathrm{a}$ & - & - & - & - & $\mathrm{n} / \mathrm{a}$ & - & - & - & - & - \\
\hline $\mathrm{Al}^{+3}$ & 0.06 & 0.08 & 0.06 & 0.04 & 0.05 & 0.10 & 0.11 & 0.09 & 0.32 & 0.16 & 0.14 \\
\hline $\mathrm{Fe}^{+3}$ & 0.06 & 0.17 & 0.14 & 0.08 & 0.19 & 0.41 & 0.36 & 0.37 & 0.05 & 0.03 & 0.03 \\
\hline $\mathrm{Mg}^{+2}$ & - & - & - & 0.06 & 0.06 & - & - & - & 0.01 & 0.02 & 0.01 \\
\hline $\mathrm{Ca}^{+2}$ & 0.02 & 0.01 & - & 0.02 & 0.01 & 0.01 & 0.01 & 0.02 & 0.01 & 0.02 & 0.02 \\
\hline $\mathrm{Ni}^{+2}$ & - & - & - & - & 0.02 & - & - & - & - & - & - \\
\hline $\mathrm{Zn}^{+2}$ & - & - & - & - & - & - & - & - & 0.02 & 0.02 & 0.01 \\
\hline $\mathrm{Sr}^{+2}$ & - & - & 0.00 & 0.02 & 0.01 & - & - & 0.02 & 0.00 & 0.01 & 0.01 \\
\hline $\mathrm{Ba}^{+2}$ & 0.70 & 0.69 & 0.72 & 0.73 & 0.75 & 0.75 & 0.79 & 0.81 & 0.61 & 0.57 & 0.54 \\
\hline $\mathrm{Pb}^{+2}$ & 0.02 & $\mathrm{n} / \mathrm{a}$ & 0.01 & n/a & $\mathrm{n} / \mathrm{a}$ & - & - & $\mathrm{n} / \mathrm{a}$ & 0.01 & 0.01 & 0.01 \\
\hline $\mathrm{Na}^{+1}$ & 0.04 & 0.03 & 0.03 & 0.04 & 0.03 & 0.04 & 0.05 & 0.05 & 0.08 & 0.04 & 0.04 \\
\hline $\mathrm{K}^{+1}$ & 0.18 & 0.12 & 0.11 & 0.17 & 0.09 & 0.07 & 0.07 & 0.07 & 0.27 & 0.33 & 0.43 \\
\hline $\mathrm{La}^{+3}$ & - & - & - & - & - & - & - & - & 0.01 & 0.01 & 0.01 \\
\hline $\mathrm{P}^{+5}$ & $\mathrm{n} / \mathrm{a}$ & n/a & $n / a$ & $\mathrm{n} / \mathrm{a}$ & $\mathrm{n} / \mathrm{a}$ & n/a & $\mathrm{n} / \mathrm{a}$ & $\mathrm{n} / \mathrm{a}$ & - & 0.01 & - \\
\hline Total & 8.56 & 8.52 & 8.53 & 8.60 & 8.58 & 8.59 & 8.61 & 8.62 & 8.69 & 8.64 & 8.69 \\
\hline $\mathrm{O}^{-2}$ & 16.00 & 16.00 & 16.00 & 16.00 & 16.00 & 16.00 & 16.00 & 16.00 & 16.00 & 16.00 & 16.00 \\
\hline
\end{tabular}

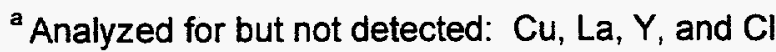

Shard-Filling Hollandite 
USW G-4 2615

Todorokite

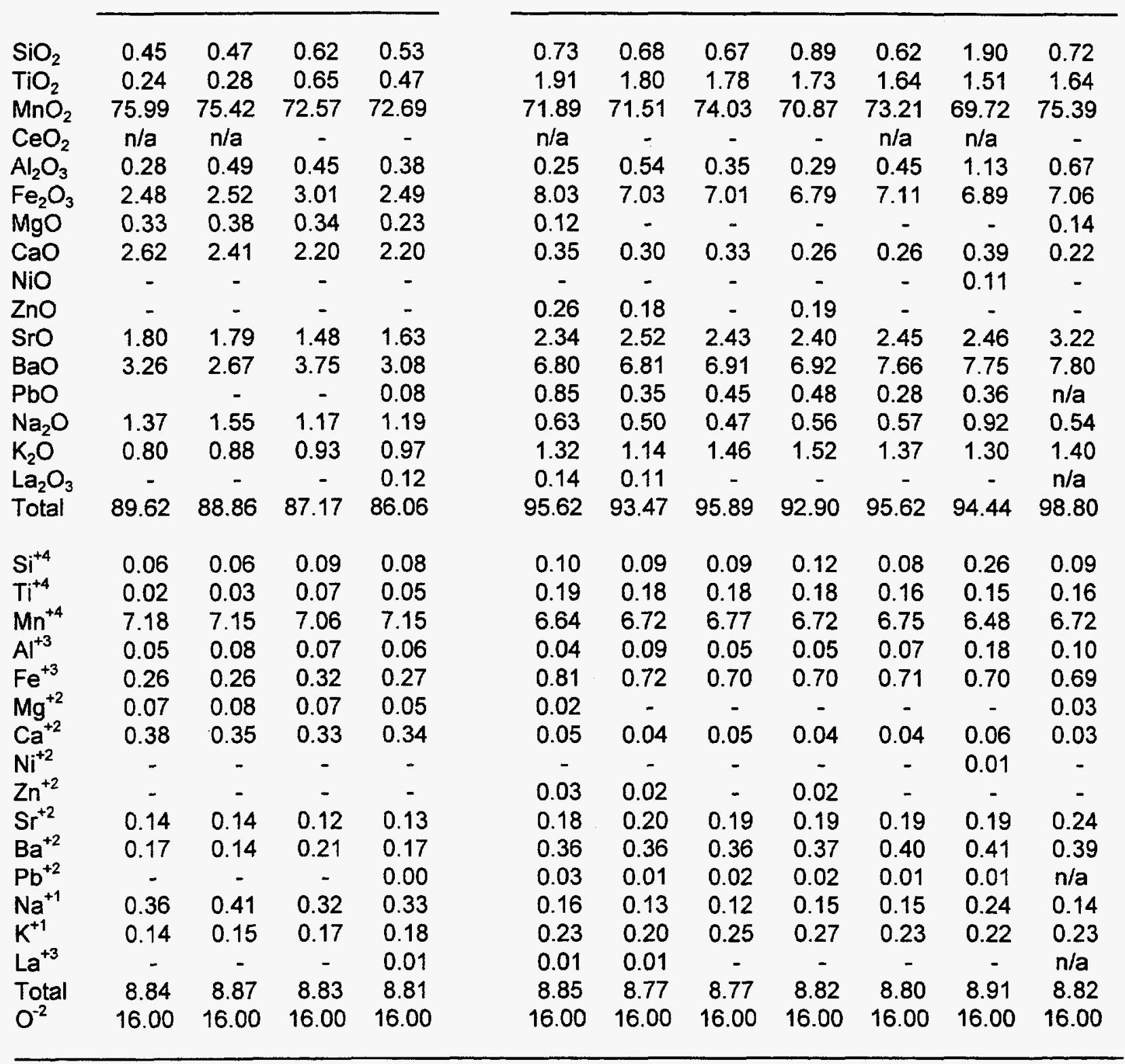

${ }^{a}$ Analyzed for but not detected: $\mathrm{Co}, \mathrm{W}, \mathrm{Cu}, \mathrm{Ag}, \mathrm{P}, \mathrm{Cl}$, and $\mathrm{Y}$

${ }^{b}$ Analyzed for but not detected: $\mathrm{P}, \mathrm{Cl}, \mathrm{Co}, \mathrm{Cu}$, and $\mathrm{Y}$

USW G-4 2620

Hollandite 
Manganese Oxides from USW G-4 2656

$\begin{array}{llllllllllllllll}\mathrm{SiO}_{2} & 0.74 & 0.69 & 0.61 & 0.64 & 0.50 & 1.25 & 0.57 & 0.81 & 1.04 & 1.00 & 0.99 & 1.18 & 1.20 & 1.23 & 1.86\end{array}$

$\begin{array}{llllllllllllllll}\mathrm{TiO}_{2} & 1.10 & 1.07 & 1.25 & 0.76 & 0.87 & 1.75 & 0.69 & 1.39 & 0.96 & 1.45 & 1.22 & 2.00 & 2.02 & 2.03 & 1.88\end{array}$

$\begin{array}{lllllllllllllllll}\mathrm{MnO}_{2} & 77.30 & 72.91 & 71.65 & 73.95 & 72.26 & 71.71 & 77.72 & 72.92 & 71.57 & 72.86 & 71.16 & 72.65 & 66.64 & 69.55 & 68.58\end{array}$

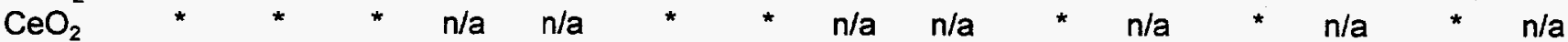

$\begin{array}{llllllllllllllll}\mathrm{Al}_{2} \mathrm{O}_{3} & 0.43 & 0.35 & 0.39 & 0.43 & 0.42 & 0.86 & 0.47 & 0.44 & 0.73 & 0.36 & 0.38 & 0.66 & 1.63 & 0.54 & 0.65\end{array}$

$\begin{array}{lllllllllllllllll}\mathrm{Fe}_{2} \mathrm{O}_{3} & 4.82 & 4.88 & 4.50 & 4.47 & 4.37 & 8.36 & 4.17 & 4.86 & 5.02 & 5.14 & 4.51 & 7.35 & 7.04 & 7.32 & 7.37\end{array}$

$\begin{array}{llllllllllllllll}\mathrm{MgO} & 3.03 & 2.92 & 2.85 & 2.76 & 2.73 & 2.76 & 2.91 & 2.66 & 2.40 & 2.47 & 2.55 & 2.19 & 1.98 & 1.78 & 1.87\end{array}$

$\begin{array}{llllllllllllllll}\mathrm{CaO} & 1.37 & 1.37 & 1.34 & 1.36 & 1.36 & 1.47 & 1.42 & 1.26 & 1.56 & 1.46 & 1.36 & 1.17 & 1.05 & 1.28 & 1.17\end{array}$

ZnO $\quad-\quad \begin{array}{llllllllllllllllll} & - & - & - & - & - & - & - & 0.24 & - & - & - & 0.18 & 0.24 & -\end{array}$

SrO $\quad \begin{array}{llllllllllllllll} & 0.63 & 0.55 & 0.56 & 0.42 & 0.44 & 0.64 & 0.60 & 0.45 & 0.46 & 0.59 & 0.40 & 0.66 & 0.52 & 0.85 & 0.49\end{array}$

$\begin{array}{lllllllllllllllll}\mathrm{BaO} & 1.48 & 1.64 & 1.90 & 2.02 & 2.03 & 2.22 & 2.30 & 2.55 & 2.68 & 2.69 & 2.85 & 2.85 & 2.98 & 3.16 & 3.27\end{array}$

$\begin{array}{llllllllllllllll}\mathrm{PbO} & \mathrm{n} / \mathrm{a} & \mathrm{n} / \mathrm{a} & \mathrm{n} / \mathrm{a} & 0.29 & 0.33 & \mathrm{n} / \mathrm{a} & \mathrm{n} / \mathrm{a} & 0.29 & 0.32 & \mathrm{n} / \mathrm{a} & 0.27 & \mathrm{n} / \mathrm{a} & 0.92 & \mathrm{n} / \mathrm{a} & 0.62\end{array}$

$\begin{array}{lllllllllllllllll}\mathrm{Na}_{2} \mathrm{O} & 1.95 & 2.31 & 1.96 & 1.88 & 2.03 & 1.50 & 2.02 & 1.90 & 1.48 & 1.94 & 1.90 & 1.38 & 1.34 & 1.56 & 1.52\end{array}$

$\begin{array}{llllllllllllllll}\mathrm{K}_{2} \mathrm{O} & 0.18 & 0.18 & 0.13 & 0.18 & 0.20 & 0.25 & 0.16 & 0.17 & 0.21 & 0.16 & 0.20 & 0.74 & 0.71 & 0.57 & 0.50\end{array}$

$\begin{array}{llllllllllllllllll}\mathrm{La}_{2} \mathrm{O}_{3} & \mathrm{n} / \mathrm{a} & \mathrm{n} / \mathrm{a} & \mathrm{n} / \mathrm{a} & 0.13 & - & \mathrm{n} / \mathrm{a} & \mathrm{n} / \mathrm{a} & - & - & \mathrm{n} / \mathrm{a} & - & \mathrm{n} / \mathrm{a} & 0.11 & \mathrm{n} / \mathrm{a} & 0.13\end{array}$

$\begin{array}{lllllllllllllllll}\text { Total } & 93.03 & 88.87 & 87.14 & 89.29 & 87.54 & 92.77 & 93.03 & 89.70 & 88.67 & 90.12 & 87.79 & 92.83 & 88.32 & 90.11 & 89.91\end{array}$

$\begin{array}{lccccccccccccccc}\mathrm{Si}^{+4} & 0.09 & 0.09 & 0.08 & 0.09 & 0.07 & 0.16 & 0.07 & 0.11 & 0.14 & 0.13 & 0.14 & 0.15 & 0.17 & 0.17 & 0.25 \\ \mathrm{Ti}^{+4} & 0.11 & 0.11 & 0.13 & 0.08 & 0.09 & 0.17 & 0.07 & 0.14 & 0.10 & 0.15 & 0.13 & 0.20 & 0.21 & 0.21 & 0.19 \\ \mathrm{Mn}^{+4} & 6.83 & 6.77 & 6.79 & 6.86 & 6.85 & 6.41 & 6.91 & 6.75 & 6.72 & 6.72 & 6.75 & 6.53 & 6.35 & 6.48 & 6.40 \\ \mathrm{Al}^{+3} & 0.06 & 0.06 & 0.06 & 0.07 & 0.07 & 0.13 & 0.07 & 0.07 & 0.12 & 0.06 & 0.06 & 0.10 & 0.26 & 0.09 & 0.10 \\ \mathrm{Fe}^{+3} & 0.46 & 0.49 & 0.46 & 0.45 & 0.45 & 0.81 & 0.40 & 0.49 & 0.51 & 0.52 & 0.47 & 0.72 & 0.73 & 0.74 & 0.75 \\ \mathrm{Mg}^{+2} & 0.58 & 0.59 & 0.58 & 0.55 & 0.56 & 0.53 & 0.56 & 0.53 & 0.49 & 0.49 & 0.52 & 0.42 & 0.41 & 0.36 & 0.38 \\ \mathrm{Ca}^{+2} & 0.19 & 0.20 & 0.20 & 0.20 & 0.20 & 0.20 & 0.20 & 0.18 & 0.23 & 0.21 & 0.20 & 0.16 & 0.16 & 0.18 & 0.17 \\ \mathrm{Zn}^{+2} & - & - & - & - & - & - & - & - & 0.02 & - & - & - & 0.02 & 0.02 & - \\ \mathrm{Sr}^{+2} & 0.05 & 0.04 & 0.04 & 0.03 & 0.04 & 0.05 & 0.04 & 0.03 & 0.04 & 0.05 & 0.03 & 0.05 & 0.04 & 0.07 & 0.04 \\ \mathrm{Ba}^{+2} & 0.07 & 0.09 & 0.10 & 0.11 & 0.11 & 0.11 & 0.12 & 0.13 & 0.14 & 0.14 & 0.15 & 0.15 & 0.16 & 0.17 & 0.17 \\ \mathrm{~Pb}^{+2} & n / a & n / a & n / a & 0.01 & 0.01 & n / a & n / a & 0.01 & 0.01 & n / a & 0.01 & n / a & 0.03 & n / a & 0.02 \\ \mathrm{Na}^{+1} & 0.48 & 0.60 & 0.52 & 0.49 & 0.54 & 0.38 & 0.50 & 0.49 & 0.39 & 0.50 & 0.51 & 0.35 & 0.36 & 0.41 & 0.40 \\ \mathrm{~K}^{+1} & 0.03 & 0.03 & 0.02 & 0.03 & 0.04 & 0.04 & 0.03 & 0.03 & 0.04 & 0.03 & 0.04 & 0.12 & 0.12 & 0.10 & 0.09 \\ \mathrm{La}^{+3} & \mathrm{n} / \mathrm{a} & n / a & n / a & 0.01 & - & \mathrm{n} / \mathrm{a} & \mathrm{n} / \mathrm{a} & - & - & \mathrm{n} / \mathrm{a} & - & \mathrm{n} / \mathrm{a} & 0.01 & \mathrm{n} / \mathrm{a} & 0.01 \\ \mathrm{Total}^{+3} & 8.96 & 9.07 & 9.00 & 8.97 & 9.02 & 9.00 & 8.97 & 8.98 & 8.94 & 8.98 & 9.00 & 8.95 & 9.02 & 8.99 & 8.97 \\ \mathrm{O}^{-2} & 16.00 & 16.00 & 16.00 & 16.00 & 16.00 & 16.00 & 16.00 & 16.00 & 16.00 & 16.00 & 16.00 & 16.00 & 16.00 & 16.00 & 16.00\end{array}$

Analyzed for but not detected: $\mathrm{P}, \mathrm{Cl}, \mathrm{Cu}, \mathrm{Mo}, \mathrm{Sc}, \mathrm{W}, \mathrm{Ta}, \mathrm{Cd}, \mathrm{Y}$, and $\mathrm{Ni}$ 
Manganese Oxides from USW G-4 2814

\begin{tabular}{lcccccc}
$\mathrm{SiO}_{2}$ & 0.97 & 1.61 & 1.54 & 0.75 & 0.88 & 0.96 \\
$\mathrm{TiO}_{2}$ & 0.10 & - & 0.10 & - & 0.10 & 0.14 \\
$\mathrm{MnO}_{2}$ & 92.77 & 90.24 & 88.62 & 91.17 & 91.93 & 89.58 \\
$\mathrm{CeO}_{2}$ & $\mathrm{n} / \mathrm{a}$ & - & $\mathrm{n} / \mathrm{a}$ & 0.19 & $\mathrm{n} / \mathrm{a}$ & $\mathrm{n} / \mathrm{a}$ \\
$\mathrm{Al}_{2} \mathrm{O}_{3}$ & 0.44 & 0.53 & 0.46 & 0.46 & 0.59 & 0.49 \\
$\mathrm{Fe}_{2} \mathrm{O}_{3}$ & 0.40 & 1.34 & 1.77 & 0.51 & 0.62 & 1.09 \\
$\mathrm{MgO}$ & - & - & - & - & - & - \\
$\mathrm{CaO}$ & 0.15 & 0.27 & 0.27 & 0.14 & 0.16 & 0.15 \\
$\mathrm{NiO}$ & - & - & - & 0.10 & - & - \\
$\mathrm{BaO}$ & 2.81 & 2.82 & 3.14 & 3.16 & 3.18 & 4.65 \\
$\mathrm{PbO}^{2}$ & - & 0.23 & 0.24 & 0.20 & 0.12 & 0.23 \\
$\mathrm{Na}_{2} \mathrm{O}$ & 0.09 & - & 0.07 & - & 0.07 & 0.08 \\
$\mathrm{~K}_{2} \mathrm{O}$ & - & - & - & - & - & - \\
$\mathrm{La}_{2} \mathrm{O}_{3}$ & - & 0.15 & - & 0.08 & - & - \\
$\mathrm{Total}^{2}$ & 97.73 & 97.19 & 96.21 & 96.76 & 97.65 & 97.37 \\
& & & & & & \\
$\mathrm{Si}^{+4}$ & 0.12 & 0.20 & 0.19 & 0.09 & 0.11 & 0.12 \\
$\mathrm{Ti}^{+4}$ & 0.01 & - & 0.01 & - & 0.01 & 0.01 \\
$\mathrm{Mn}^{+4}$ & 7.72 & 7.56 & 7.53 & 7.72 & 7.69 & 7.61 \\
$\mathrm{Ce}^{+4}$ & $n / a$ & - & $n / a$ & 0.01 & $n / a$ & $n / a$ \\
$\mathrm{Al}^{+3}$ & 0.06 & 0.08 & 0.07 & 0.07 & 0.08 & 0.07 \\
$\mathrm{Fe}^{+3}$ & 0.04 & 0.12 & 0.16 & 0.05 & 0.06 & 0.10 \\
$\mathrm{Mg}^{+2}$ & - & - & - & - & - & - \\
$\mathrm{Ca}^{+2}$ & 0.02 & 0.04 & 0.04 & 0.02 & 0.02 & 0.02 \\
$\mathrm{Ni}^{+2}$ & - & - & - & 0.01 & - & - \\
$\mathrm{Ba}^{+2}$ & 0.13 & 0.13 & 0.15 & 0.15 & 0.15 & 0.22 \\
$\mathrm{~Pb}^{+2}$ & - & 0.01 & 0.01 & 0.01 & 0.00 & 0.01 \\
$\mathrm{Na}^{+1}$ & 0.02 & - & 0.02 & - & 0.02 & 0.02 \\
$\mathrm{~K}^{+1}$ & - & - & - & - & - & - \\
$\mathrm{La}^{+3}$ & - & 0.01 & - & 0.00 & - & - \\
$\mathrm{Total}^{+3}$ & 8.12 & 8.14 & 8.17 & 8.12 & 8.14 & 8.18 \\
$\mathrm{O}^{-2}$ & 16.00 & 16.00 & 16.00 & 16.00 & 16.00 & 16.00 \\
& & & & & & \\
\hline & & & & & & \\
\hline
\end{tabular}

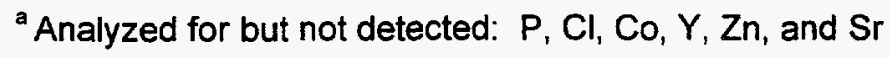




\begin{tabular}{|c|c|c|c|c|c|c|c|c|c|c|c|c|}
\hline $\mathrm{SiO}_{2}$ & 0.97 & 1.70 & 0.87 & 1.02 & 0.85 & 0.77 & 1.22 & 0.52 & 1.66 & 0.28 & 0.42 & 0.51 \\
\hline $\mathrm{TiO}_{2}$ & 0.43 & 0.24 & 0.30 & 0.66 & 0.68 & 0.34 & 0.73 & - & 0.15 & 0.15 & - & 0.11 \\
\hline $\mathrm{MnO}_{2}$ & 84.08 & 83.00 & 84.33 & 74.62 & 75.98 & 79.12 & 66.54 & 82.21 & 71.45 & 77.57 & 79.40 & 82.41 \\
\hline $\mathrm{CeO}_{2}$ & ** & ** & $\mathrm{n} / \mathrm{a}$ & n/a & * & $\mathrm{n} / \mathrm{a}$ & n/a & $\mathrm{n} / \mathrm{a}$ & $\mathrm{n} / \mathrm{a}$ & 0.50 & n/a & $\mathrm{n} / \mathrm{a}$ \\
\hline $\mathrm{Al}_{2} \mathrm{O}_{3}$ & 1.20 & 1.24 & 0.76 & 0.98 & 1.47 & 0.67 & 1.16 & 0.69 & 0.94 & 0.32 & 0.42 & 0.74 \\
\hline $\mathrm{Fe}_{2} \mathrm{O}_{3}$ & 2.92 & 2.60 & 2.10 & 4.90 & 4.44 & 3.16 & 12.75 & 0.86 & 6.22 & 0.81 & 0.68 & 0.80 \\
\hline $\mathrm{MgO}$ & - & - & - & - & 0.38 & 0.05 & - & - & 0.12 & 0.22 & 0.09 & - \\
\hline $\mathrm{CaO}$ & 0.35 & 0.36 & 0.61 & 0.40 & 0.37 & 0.64 & 0.31 & 0.78 & 0.97 & 0.72 & 0.78 & 0.70 \\
\hline $\mathrm{ZnO}$ & - & - & 0.18 & - & - & - & - & - & 0.25 & 0.36 & 0.30 & - \\
\hline SrO & - & 0.15 & 0.28 & 0.15 & 0.24 & 0.93 & 0.14 & 0.78 & 0.88 & 1.09 & 1.60 & 0.60 \\
\hline $\mathrm{BaO}$ & 1.46 & 1.84 & 3.86 & 5.38 & 5.59 & 6.99 & 7.69 & 8.30 & 8.69 & 8.88 & 8.94 & 8.98 \\
\hline $\mathrm{PbO}$ & $\mathrm{n} / \mathrm{a}$ & $\mathrm{n} / \mathrm{a}$ & 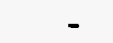 & 0.18 & n/a & 0.24 & 0.28 & 0.52 & 0.56 & 0.35 & 0.17 & 0.65 \\
\hline $\mathrm{Na}_{2} \mathrm{O}$ & - & - & 0.18 & 0.08 & - & 0.30 & 0.09 & 0.37 & 0.43 & 0.48 & 0.64 & 0.37 \\
\hline $\mathrm{K}_{2} \mathrm{O}$ & - & - & 0.16 & 0.14 & 0.09 & 0.45 & 0.12 & 0.63 & 0.64 & 0.35 & 0.79 & 0.53 \\
\hline $\mathrm{La}_{2} \mathrm{O}_{3}$ & n/a & $n / a$ & - & 0.16 & $\mathrm{n} / \mathrm{a}$ & 0.12 & - & - & - & - & 0.13 & - \\
\hline $\mathrm{P}_{2} \mathrm{O}_{5}$ & $\mathrm{n} / \mathrm{a}$ & $\mathrm{n} / \mathrm{a}$ & - & - & n/a & - & 0.06 & - & - & - & - & - \\
\hline Total & 91.41 & 91.13 & 93.63 & 88.67 & 90.09 & 93.78 & 91.09 & 95.66 & 92.96 & 92.08 & 94.36 & 96.40 \\
\hline $\mathrm{Si}^{+4}$ & 0.12 & 0.22 & 0.11 & 0.14 & 0.11 & 0.10 & 0.17 & 0.07 & 0.23 & 0.04 & 0.06 & 0.07 \\
\hline $\mathrm{Ti}^{+4}$ & 0.04 & 0.02 & 0.03 & 0.07 & 0.07 & 0.03 & 0.08 & - & 0.02 & 0.02 & - & 0.01 \\
\hline $\mathrm{Mn}^{+4}$ & 7.43 & 7.35 & 7.45 & 7.09 & 7.08 & 7.24 & 6.36 & 7.43 & 6.74 & 7.39 & 7.38 & 7.42 \\
\hline $\mathrm{Ce}^{+4}$ & - & - & $\mathrm{n} / \mathrm{a}$ & $\mathrm{n} / \mathrm{a}$ & - & $\mathrm{n} / \mathrm{a}$ & $\mathrm{n} / \mathrm{a}$ & $\mathrm{n} / \mathrm{a}$ & $\mathrm{n} / \mathrm{a}$ & 0.02 & $\mathrm{n} / \mathrm{a}$ & $\mathrm{n} / \mathrm{a}$ \\
\hline $\mathrm{Al}^{+3}$ & 0.18 & 0.19 & 0.11 & 0.16 & 0.23 & 0.10 & 0.19 & 0.11 & 0.15 & 0.05 & 0.07 & 0.11 \\
\hline $\mathrm{Fe}^{+3}$ & 0.28 & 0.25 & 0.20 & 0.51 & 0.45 & 0.31 & 1.33 & 0.08 & 0.64 & 0.08 & 0.07 & 0.08 \\
\hline $\mathrm{Mg}^{+2}$ & - & - & - & - & 0.08 & 0.01 & - & - & 0.02 & 0.05 & 0.02 & - \\
\hline $\mathrm{Ca}^{+2}$ & 0.05 & 0.05 & 0.08 & 0.06 & 0.05 & 0.09 & 0.05 & 0.11 & 0.14 & 0.11 & 0.11 & 0.10 \\
\hline $\mathrm{Zn}^{+2}$ & - & - & 0.02 & - & - & - & - & - & 0.03 & 0.04 & 0.03 & - \\
\hline $\mathrm{Sr}^{+2}$ & - & 0.01 & 0.02 & 0.01 & 0.02 & 0.07 & 0.01 & 0.06 & 0.07 & 0.09 & 0.12 & 0.05 \\
\hline $\mathrm{Ba}^{+2}$ & 0.07 & 0.09 & 0.19 & 0.29 & 0.30 & 0.36 & 0.42 & 0.43 & 0.46 & 0.48 & 0.47 & 0.46 \\
\hline $\mathrm{Pb}^{+2}$ & $\mathrm{n} / \mathrm{a}$ & $\mathrm{n} / \mathrm{a}$ & - & 0.01 & $\mathrm{n} / \mathrm{a}$ & 0.01 & 0.01 & 0.02 & 0.02 & 0.01 & 0.01 & 0.02 \\
\hline $\mathrm{Na}^{+1}$ & - & - & 0.04 & 0.02 & - & 0.08 & 0.02 & 0.09 & 0.11 & 0.13 & 0.17 & 0.09 \\
\hline $\mathrm{K}^{+1}$ & - & - & 0.03 & 0.02 & 0.02 & 0.08 & 0.02 & 0.11 & 0.11 & 0.06 & 0.14 & 0.09 \\
\hline $\mathrm{La}^{+3}$ & $\mathrm{n} / \mathrm{a}$ & $\mathrm{n} / \mathrm{a}$ & - & 0.01 & $\mathrm{n} / \mathrm{a}$ & 0.01 & - & - & - & - & 0.01 & - \\
\hline $\mathrm{P}^{+5}$ & $\mathrm{n} / \mathrm{a}$ & $\mathrm{n} / \mathrm{a}$ & - & - & $\mathrm{n} / \mathrm{a}$ & - & 0.01 & - & - & - & - & \\
\hline $\begin{array}{l}\text { Total } \\
\mathrm{O}^{-2}\end{array}$ & $\begin{array}{r}8.18 \\
16.00\end{array}$ & $\begin{array}{r}8.19 \\
16.00\end{array}$ & $\begin{array}{r}8.29 \\
16.00\end{array}$ & $\begin{array}{r}8.39 \\
16.00\end{array}$ & $\begin{array}{r}8.40 \\
16.00\end{array}$ & $\begin{array}{r}8.49 \\
16.00\end{array}$ & $\begin{array}{r}8.65 \\
16.00\end{array}$ & $\begin{array}{r}8.50 \\
16.00\end{array}$ & $\begin{array}{r}8.74 \\
16.00\end{array}$ & $\begin{array}{r}8.56 \\
16.00\end{array}$ & $\begin{array}{r}8.64 \\
16.00\end{array}$ & $\begin{array}{r}8.50 \\
16.00\end{array}$ \\
\hline
\end{tabular}


Manganese Oxides from USW G-4 2854, continued

\begin{tabular}{|c|c|c|c|c|c|c|c|c|c|c|c|c|}
\hline $\mathrm{SiO}_{2}$ & 1.20 & 1.24 & 0.38 & 0.41 & 0.39 & 0.40 & 0.42 & 0.34 & 0.42 & 0.32 & 0.39 & 0.52 \\
\hline $\mathrm{TiO}_{2}$ & 1.00 & 0.57 & 0.37 & - & 0.29 & - & 0.14 & 0.34 & - & 0.13 & - & 0.87 \\
\hline $\mathrm{MnO}_{2}$ & 74.21 & 67.82 & 77.34 & 79.25 & 77.52 & 77.73 & 78.62 & 74.78 & 77.52 & 78.54 & 74.20 & 72.11 \\
\hline $\mathrm{CeO}_{2}$ & * & n/a & 0.66 & n/a & n/a & $\mathrm{n} / \mathrm{a}$ & $\mathrm{n} / \mathrm{a}$ & $\mathrm{n} / \mathrm{a}$ & $\mathrm{n} / \mathrm{a}$ & $\mathrm{n} / \mathrm{a}$ & 0.36 & 0.57 \\
\hline $\mathrm{Al}_{2} \mathrm{O}_{3}$ & 1.67 & 0.78 & 0.38 & 0.43 & 0.41 & 0.45 & 0.37 & 0.43 & 0.40 & 0.42 & 0.36 & 0.44 \\
\hline $\mathrm{Fe}_{2} \mathrm{O}_{3}$ & 6.53 & 8.85 & 2.17 & 1.01 & 1.98 & 1.08 & 1.15 & 1.98 & 1.40 & 0.63 & 1.43 & 6.11 \\
\hline $\mathrm{MgO}$ & - & 0.08 & 0.11 & 0.07 & 0.14 & 0.14 & 0.21 & 0.10 & 0.21 & 0.12 & 0.27 & 0.08 \\
\hline $\mathrm{CaO}$ & 0.36 & 0.73 & 0.62 & 0.72 & 0.72 & 0.77 & 0.94 & 0.69 & 0.89 & 0.87 & 0.91 & 0.49 \\
\hline $\mathrm{ZnO}$ & - & - & 0.27 & 0.24 & 0.18 & 0.18 & 0.29 & - & 0.36 & 0.23 & 0.33 & - \\
\hline SrO & 0.43 & 0.60 & 1.45 & 1.46 & 1.51 & 1.33 & 1.03 & 1.36 & 0.92 & 1.28 & 0.88 & 0.83 \\
\hline $\mathrm{BaO}$ & 9.02 & 9.06 & 9.16 & 9.17 & 9.27 & 9.27 & 9.79 & 9.81 & 9.82 & 10.11 & 10.13 & 12.28 \\
\hline $\mathrm{PbO}$ & $\mathrm{n} / \mathrm{a}$ & 0.72 & 0.15 & - & - & 0.18 & 0.52 & 0.14 & 0.64 & 0.40 & 0.51 & 0.17 \\
\hline $\mathrm{Na}_{2} \mathrm{O}$ & 0.14 & 0.26 & 0.55 & 0.54 & 0.61 & 0.46 & 0.53 & 0.56 & 0.48 & 0.53 & 0.53 & 0.38 \\
\hline $\mathrm{K}_{2} \mathrm{O}^{\circ}$ & 0.20 & 0.35 & 0.30 & 0.63 & 0.62 & 0.80 & 0.59 & 0.61 & 0.33 & 0.64 & 0.29 & 0.22 \\
\hline $\mathrm{La}_{2} \mathrm{O}_{3}$ & $\mathrm{n} / \mathrm{a}$ & - & - & - & - & 0.11 & - & - & 0.10 & - & 0.18 & 0.13 \\
\hline $\mathrm{P}_{2} \mathrm{O}_{5}$ & n/a & - & - & - & - & - & 0.17 & - & 0.17 & 0.07 & 0.13 & - \\
\hline Total & 94.76 & 91.06 & 93.91 & 93.93 & 93.64 & 92.90 & 94.77 & 91.14 & 93.66 & 94.29 & 90.90 & 95.20 \\
\hline $\mathrm{Si}^{+4}$ & 0.16 & 0.17 & 0.05 & 0.06 & 0.05 & 0.05 & 0.06 & 0.05 & 0.06 & 0.04 & 0.06 & 0.07 \\
\hline $\mathrm{Ti}^{+4}$ & 0.10 & 0.06 & 0.04 & - & 0.03 & - & 0.01 & 0.04 & - & 0.01 & - & 0.09 \\
\hline $\mathrm{Mn}^{+4}$ & 6.77 & 6.58 & 7.25 & 7.38 & 7.27 & 7.35 & 7.30 & 7.25 & 7.30 & 7.36 & 7.25 & 6.84 \\
\hline $\mathrm{Ce}^{+4}$ & - & $n / a$ & 0.03 & $\mathrm{n} / \mathrm{a}$ & $n / a$ & $\mathrm{n} / \mathrm{a}$ & $\mathrm{n} / \mathrm{a}$ & $\mathrm{n} / \mathrm{a}$ & $\mathrm{n} / \mathrm{a}$ & $\mathrm{n} / \mathrm{a}$ & 0.02 & 0.03 \\
\hline $\mathrm{Al}^{+3}$ & 0.26 & 0.13 & 0.06 & 0.07 & 0.07 & 0.07 & 0.06 & 0.07 & 0.06 & 0.07 & 0.06 & 0.07 \\
\hline $\mathrm{Fe}^{+3}$ & 0.65 & 0.94 & 0.22 & 0.10 & 0.20 & 0.11 & 0.12 & 0.21 & 0.14 & 0.06 & 0.15 & 0.63 \\
\hline $\mathrm{Mg}^{+2}$ & - & 0.02 & 0.02 & 0.01 & 0.03 & 0.03 & 0.04 & 0.02 & 0.04 & 0.02 & 0.06 & 0.02 \\
\hline $\mathrm{Ca}^{+2}$ & 0.05 & 0.11 & 0.09 & 0.10 & 0.10 & 0.11 & 0.14 & 0.10 & 0.13 & 0.13 & 0.14 & 0.07 \\
\hline $\mathrm{Zn}^{+2}$ & - & - & 0.03 & 0.02 & 0.02 & 0.02 & 0.03 & - & 0.04 & 0.02 & 0.03 & - \\
\hline $\mathrm{Sr}^{+2}$ & 0.03 & 0.05 & 0.11 & 0.11 & 0.12 & 0.11 & 0.08 & 0.11 & 0.07 & 0.10 & 0.07 & 0.07 \\
\hline $\mathrm{Ba}^{+2}$ & 0.47 & 0.50 & 0.49 & 0.48 & 0.49 & 0.50 & 0.52 & 0.54 & 0.52 & 0.54 & 0.56 & 0.66 \\
\hline $\mathrm{Pb}^{+2}$ & $\mathrm{n} / \mathrm{a}$ & 0.03 & 0.01 & - & - & 0.01 & 0.02 & 0.01 & 0.02 & 0.01 & 0.02 & 0.01 \\
\hline $\mathrm{Na}^{+1}$ & 0.04 & 0.07 & 0.14 & 0.14 & 0.16 & 0.12 & 0.14 & 0.15 & 0.13 & 0.14 & 0.15 & 0.10 \\
\hline $\mathrm{K}^{+1}$ & 0.03 & 0.06 & 0.05 & 0.11 & 0.11 & 0.14 & 0.10 & 0.11 & 0.06 & 0.11 & 0.05 & 0.04 \\
\hline $\mathrm{La}^{+3}$ & $\mathrm{n} / \mathrm{a}$ & - & - & - & - & 0.01 & - & - & 0.01 & - & 0.01 & 0.01 \\
\hline $\mathrm{P}^{+5}$ & $n / a$ & - & - & - & - & - & 0.02 & - & 0.02 & 0.01 & 0.02 & - \\
\hline $\begin{array}{l}\text { Total } \\
\mathrm{O}^{-2}\end{array}$ & $\begin{array}{r}8.55 \\
16.00\end{array}$ & $\begin{array}{r}8.72 \\
16.00\end{array}$ & $\begin{array}{r}8.59 \\
16.00\end{array}$ & $\begin{array}{r}8.60 \\
16.00\end{array}$ & $\begin{array}{r}8.65 \\
16.00\end{array}$ & $\begin{array}{r}8.63 \\
16.00\end{array}$ & $\begin{array}{r}8.63 \\
16.00\end{array}$ & $\begin{array}{r}8.66 \\
16.00\end{array}$ & $\begin{array}{r}8.60 \\
16.00\end{array}$ & $\begin{array}{r}8.63 \\
16.00\end{array}$ & $\begin{array}{r}8.64 \\
16.00\end{array}$ & $\begin{array}{r}8.69 \\
16.00\end{array}$ \\
\hline
\end{tabular}


Manganese Oxides from USW G-4 2854, continued

\begin{tabular}{|c|c|c|c|c|c|c|c|c|}
\hline \multirow[b]{2}{*}{$\mathrm{SiO}_{2}$} & \multirow[b]{2}{*}{0.62} & \multicolumn{3}{|c|}{ W-Hollandite } & \multicolumn{2}{|c|}{ Pyrolusite } & \multicolumn{2}{|c|}{ Todorokite? } \\
\hline & & 0.44 & 0.37 & 0.21 & 0.99 & 1.19 & 0.46 & 0.63 \\
\hline $\mathrm{TiO}_{2}$ & 0.73 & 0.99 & 0.84 & 0.90 & 0.29 & 0.34 & 0.20 & 0.72 \\
\hline $\mathrm{MnO}_{2}$ & 70.44 & 63.15 & 64.71 & 65.84 & 87.87 & 88.20 & 77.17 & 69.00 \\
\hline $\mathrm{CeO}_{2}$ & n/a & - & - & - & 0.39 & 0.87 & 0.56 & 0.29 \\
\hline $\mathrm{Al}_{2} \mathrm{O}_{3}$ & 0.59 & 1.20 & 0.94 & 0.90 & 2.30 & 0.84 & 0.51 & 0.48 \\
\hline $\mathrm{Fe}_{2} \mathrm{O}_{3}$ & 6.26 & 8.51 & 8.33 & 8.62 & 2.28 & 2.25 & 2.80 & 8.38 \\
\hline $\mathrm{MgO}$ & 0.08 & 0.06 & 0.07 & 0.06 & 0.04 & 0.05 & 0.10 & 0.09 \\
\hline $\mathrm{CaO}$ & 0.40 & 0.11 & 0.15 & 0.13 & 0.48 & 0.47 & 0.77 & 0.43 \\
\hline $\mathrm{ZnO}$ & - & - & 0.22 & 0.31 & - & 0.16 & 0.33 & 0.26 \\
\hline SrO & 0.54 & - & - & - & 0.15 & 0.23 & 1.18 & 0.39 \\
\hline $\mathrm{BaO}$ & 13.13 & 13.95 & 13.62 & 14.69 & 2.36 & 2.08 & 8.85 & 12.43 \\
\hline $\mathrm{PbO}$ & 0.23 & 0.42 & 0.35 & 0.16 & 0.13 & 0.19 & 0.17 & 0.20 \\
\hline $\mathrm{Na}_{2} \mathrm{O}$ & 0.25 & 0.08 & 0.11 & - & - & 0.19 & 0.51 & 0.20 \\
\hline $\mathrm{K}_{2} \mathrm{O}$ & 0.31 & 0.09 & 0.11 & 0.10 & 0.09 & 0.15 & 0.61 & 0.27 \\
\hline $\mathrm{La}_{2} \mathrm{O}_{3}$ & - & - & 0.12 & - & 0.12 & 0.15 & - & 0.10 \\
\hline $\mathrm{WO}_{3}$ & n/a & 2.00 & 2.62 & 2.89 & - & - & - & 0.50 \\
\hline $\mathrm{P}_{2} \mathrm{O}_{5}$ & - & $\mathrm{n} / \mathrm{a}$ & $\mathrm{n} / \mathrm{a}$ & $\mathrm{n} / \mathrm{a}$ & $\mathrm{n} / \mathrm{a}$ & n/a & $n / a$ & $\mathrm{n} / \mathrm{a}$ \\
\hline Total & 93.58 & 91.00 & 92.66 & 94.81 & 97.49 & 97.36 & 94.22 & 94.37 \\
\hline $\mathrm{Si}^{+4}$ & 0.09 & 0.06 & 0.05 & 0.03 & 0.12 & 0.14 & 0.06 & 0.09 \\
\hline $\mathrm{Ti}^{+4}$ & 0.08 & 0.11 & 0.09 & 0.10 & 0.03 & 0.03 & 0.02 & 0.08 \\
\hline $\mathrm{Mn}^{+4}$ & 6.81 & 6.41 & 6.46 & 6.46 & 7.33 & 7.42 & 7.19 & 6.64 \\
\hline $\mathrm{Ce}^{+4}$ & $\mathrm{n} / \mathrm{a}$ & - & - & - & 0.02 & 0.04 & 0.03 & 0.01 \\
\hline $\mathrm{Al}^{+3}$ & 0.10 & 0.21 & 0.16 & 0.15 & 0.33 & 0.12 & 0.08 & 0.08 \\
\hline $\mathrm{Fe}^{+3}$ & 0.66 & 0.94 & 0.91 & 0.92 & 0.21 & 0.21 & 0.28 & 0.88 \\
\hline $\mathrm{Mg}^{+2}$ & 0.02 & 0.01 & 0.02 & 0.01 & 0.01 & 0.01 & 0.02 & 0.02 \\
\hline $\mathrm{Ca}^{+2}$ & 0.06 & 0.02 & 0.02 & 0.02 & 0.06 & 0.06 & 0.11 & 0.06 \\
\hline $\mathrm{Zn}^{+2}$ & - & - & 0.02 & 0.03 & - & 0.01 & 0.03 & 0.03 \\
\hline $\mathrm{Sr}^{+2}$ & 0.04 & - & - & - & 0.01 & 0.02 & 0.09 & 0.03 \\
\hline $\mathrm{Ba}^{+2}$ & 0.72 & 0.80 & 0.77 & 0.82 & 0.11 & 0.10 & 0.47 & 0.68 \\
\hline $\mathrm{Pb}^{+2}$ & 0.01 & 0.02 & 0.01 & 0.01 & 0.00 & 0.01 & 0.01 & 0.01 \\
\hline $\mathrm{Na}^{+1}$ & 0.07 & 0.02 & 0.03 & - & - & 0.04 & 0.13 & 0.05 \\
\hline $\mathrm{K}^{+1}$ & 0.06 & 0.02 & 0.02 & 0.02 & 0.01 & 0.02 & 0.10 & 0.05 \\
\hline $\mathrm{La}^{+3}$ & - & - & 0.01 & - & 0.01 & 0.01 & - & 0.01 \\
\hline$W^{+6}$ & $\mathrm{n} / \mathrm{a}$ & 0.08 & 0.10 & 0.11 & - & - & - & 0.02 \\
\hline $\mathrm{P}^{+5}$ & - & $\mathrm{n} / \mathrm{a}$ & $n / a$ & $\mathrm{n} / \mathrm{a}$ & $n / a$ & $n / a$ & $\mathrm{n} / \mathrm{a}$ & $\mathrm{n} / \mathrm{a}$ \\
\hline Total & 8.71 & 8.70 & 8.69 & 8.67 & 8.24 & 8.24 & 8.64 & 8.72 \\
\hline $\mathrm{O}^{-2}$ & 16.00 & 16.00 & 16.00 & 16.00 & 16.00 & 16.00 & 16.00 & 16.00 \\
\hline
\end{tabular}

analyzed for but not detected: $\mathrm{Cl}, \mathrm{Y}$, and $\mathrm{Ni}$ 
Blades

\begin{tabular}{|c|c|c|c|c|c|c|c|c|c|c|c|c|c|c|}
\hline $\mathrm{SiO}_{2}$ & 1.17 & 1.56 & 0.89 & 1.08 & 1.13 & 0.40 & 0.53 & 1.37 & 0.46 & 0.44 & 0.64 & 0.86 & 0.79 & 0.28 \\
\hline & 0.33 & 0.31 & 0.61 & 0.66 & 0.78 & 0.49 & 0.61 & 1.68 & 0.34 & 0.34 & 0.84 & 1.60 & 1.84 & 0.25 \\
\hline $\mathrm{nO}_{2}$ & 86.95 & 83.33 & 81.57 & 80.57 & 79.50 & 74.63 & 75.03 & 61.77 & 77.85 & 79.28 & 74.49 & 64.44 & 68.29 & 80.53 \\
\hline $\mathrm{eO}_{2}$ & 0.39 & 0.35 & - & 0.33 & - & 0.17 & - & 0.27 & 0.37 & 0.41 & 0.29 & 0.24 & - & 0.20 \\
\hline $\mathrm{l}_{2} \mathrm{O}_{3}$ & 0.79 & 0.78 & 0.61 & 0.67 & 1.07 & 0.35 & 0.37 & 0.58 & 0.51 & 0.50 & 0.58 & 0.64 & 0.69 & 0.49 \\
\hline $\mathrm{e}_{2} \mathrm{O}_{3}$ & 2.32 & 2.93 & 3.82 & 3.65 & 4.05 & 3.68 & 3.99 & 8.66 & 2.91 & 2.65 & 4.79 & 6.03 & 6.15 & 1.31 \\
\hline go & 0.08 & 0.09 & 0.06 & 0.13 & 0.09 & 0.18 & 0.17 & 0.16 & 0.11 & 0.10 & 0.10 & 0.10 & 0.10 & 0.12 \\
\hline $\mathrm{aO}$ & 0.18 & 0.16 & 0.23 & 0.27 & 0.25 & 1.41 & 1.33 & 0.92 & 0.79 & 0.74 & 0.54 & 0.71 & 0.66 & 0.66 \\
\hline & - & - & - & 0.09 & - & - & - & - & - & - & - & - & - & - \\
\hline no & 0.43 & 0.58 & - & 0.47 & 0.39 & 0.28 & - & 0.35 & 0.18 & 0.18 & 0.19 & - & - & 0.16 \\
\hline ro & - & - & - & 0.13 & 0.12 & 1.99 & 1.87 & 1.26 & 1.33 & 1.26 & 1.26 & 1.03 & 1.02 & 1.13 \\
\hline $\mathrm{aO}$ & - & 0.18 & 1.50 & 1.72 & 1.92 & 4.51 & 4.54 & 6.77 & 7.10 & 7.34 & 7.49 & 7.60 & 7.68 & 8.50 \\
\hline $\mathrm{boO}$ & - & - & - & 0.15 & 0.16 & 0.21 & 0.29 & 0.68 & 0.26 & 0.30 & 0.32 & 0.39 & 0.34 & 0.29 \\
\hline $\mathrm{la}_{2} \mathrm{O}$ & 0.12 & 0.17 & 0.11 & 0.19 & - & 0.92 & 0.73 & 0.40 & 0.57 & 0.41 & 0.38 & 0.32 & 0.37 & 0.42 \\
\hline $\mathrm{C}_{2} \mathrm{O}$ & - & - & 0.15 & 0.10 & 0.16 & 1.36 & 1.13 & 0.72 & 1.84 & 1.66 & 1.78 & 1.65 & 1.64 & 1.78 \\
\hline$a_{2} O_{3}$ & - & 0.11 & - & 0.11 & 0.08 & 0.19 & 0.19 & 0.14 & 0.25 & 0.19 & 0.25 & 0.12 & 0.16 & 0.20 \\
\hline${ }_{2} \mathrm{O}_{5}$ & - & - & - & - & - & - & - & - & - & - & - & - & - & 0.08 \\
\hline otal & 92.76 & 90.55 & 89.55 & 90.32 & 89.70 & 90.77 & 90.78 & 85.73 & 94.87 & 95.80 & 93.94 & 85.73 & 89.73 & 96.40 \\
\hline $\mathrm{Si}^{+4}$ & 0.15 & 0.20 & 0.12 & 0.14 & 0.15 & 0.05 & 0.07 & 0.20 & 0.06 & 0.06 & 0.09 & 0.13 & 0.11 & 0.04 \\
\hline $\mathrm{Ti}^{+4}$ & 0.03 & 0.03 & 0.06 & 0.07 & 0.08 & 0.05 & 0.06 & 0.19 & 0.03 & 0.03 & 0.09 & 0.18 & 0.20 & 0.02 \\
\hline $\mathrm{Mn}^{+4}$ & 7.51 & 7.39 & 7.40 & 7.30 & 7.24 & 7.09 & 7.09 & 6.35 & 7.17 & 7.22 & 6.96 & 6.64 & 6.69 & 7.32 \\
\hline $\mathrm{Ce}^{+4}$ & 0.02 & 0.02 & - & 0.02 & - & 0.01 & - & 0.01 & 0.02 & 0.02 & 0.01 & 0.01 & - & 0.01 \\
\hline $\mathrm{Al}^{+3}$ & 0.12 & 0.12 & 0.09 & 0.10 & 0.17 & 0.06 & 0.06 & 0.10 & 0.08 & 0.08 & 0.09 & 0.11 & 0.12 & 0.08 \\
\hline $\mathrm{Fe}^{+3}$ & 0.22 & 0.28 & 0.38 & 0.36 & 0.40 & 0.38 & 0.41 & 0.97 & 0.29 & 0.26 & 0.49 & 0.68 & 0.66 & 0.13 \\
\hline $\mathrm{Mg}^{+2}$ & 0.01 & 0.02 & 0.01 & 0.03 & 0.02 & 0.04 & 0.03 & 0.04 & 0.02 & 0.02 & 0.02 & 0.02 & 0.02 & 0.02 \\
\hline $\mathrm{Ca}^{+2}$ & 0.02 & 0.02 & 0.03 & 0.04 & 0.04 & 0.21 & 0.19 & 0.15 & 0.11 & 0.10 & 0.08 & 0.11 & 0.10 & 0.09 \\
\hline $\mathrm{Ni}^{+2}$ & & - & - & 0.01 & - & - & - & - & - & - & - & - & - & - \\
\hline $\mathrm{Zn}^{+2}$ & 0.04 & 0.05 & - & 0.05 & 0.04 & 0.03 & - & 0.04 & 0.02 & 0.02 & 0.02 & - & - & 0.02 \\
\hline $\mathrm{Sr}^{+2}$ & - & - & - & 0.01 & 0.01 & 0.16 & 0.15 & 0.11 & 0.10 & 0.10 & 0.10 & 0.09 & 0.08 & 0.09 \\
\hline $\mathrm{Ba}^{+2}$ & - & 0.01 & 0.08 & 0.09 & 0.10 & 0.24 & 0.24 & 0.39 & 0.37 & 0.38 & 0.40 & 0.44 & 0.43 & 0.44 \\
\hline $\mathrm{Pb}^{+2}$ & - & - & - & 0.01 & 0.01 & 0.01 & 0.01 & 0.03 & 0.01 & 0.01 & 0.01 & 0.02 & 0.01 & 0.01 \\
\hline $\mathrm{Na}^{+1}$ & 0.03 & 0.04 & 0.03 & 0.05 & - & 0.25 & 0.19 & 0.12 & 0.15 & 0.10 & 0.10 & 0.09 & 0.10 & 0.11 \\
\hline $\mathrm{K}^{+1}$ & - & - & 0.03 & 0.02 & 0.03 & 0.24 & 0.20 & 0.14 & 0.31 & 0.28 & 0.31 & 0.31 & 0.30 & 0.30 \\
\hline $\mathrm{La}^{+3}$ & - & 0.01 & - & 0.01 & 0.00 & 0.01 & 0.01 & 0.01 & .01 & 0.01 & 0.01 & 0.01 & 0.01 & 0.01 \\
\hline $\mathrm{P}^{+5}$ & - & - & - & - & - & - & - & - & - & - & - & - & - & 0.01 \\
\hline $\begin{array}{l}\text { Total } \\
\mathrm{O}^{-2}\end{array}$ & $\begin{array}{r}8.14 \\
16.00\end{array}$ & $\begin{array}{r}8.18 \\
16.00\end{array}$ & $\begin{array}{r}8.22 \\
16.00\end{array}$ & $\begin{array}{r}8.28 \\
16.00\end{array}$ & $\begin{array}{r}8.27 \\
16.00\end{array}$ & $\begin{array}{r}8.82 \\
16.00\end{array}$ & $\begin{array}{r}8.73 \\
16.00\end{array}$ & $\begin{array}{r}8.83 \\
16.00\end{array}$ & $\begin{array}{r}8.76 \\
16.00\end{array}$ & $\begin{array}{r}8.69 \\
16.00\end{array}$ & $\begin{array}{r}8.76 \\
16.00\end{array}$ & $\begin{array}{r}8.85 \\
16.00\end{array}$ & $\begin{array}{r}8.82 \\
16.00\end{array}$ & $\begin{array}{r}8.69 \\
16.00\end{array}$ \\
\hline
\end{tabular}

\footnotetext{
analyzed for but not detected: Co
}

Lobes 
Hollandite

\begin{tabular}{|c|c|c|c|c|c|c|c|c|c|}
\hline $\mathrm{SiO}_{2}$ & 1.06 & 1.02 & 1.12 & 1.02 & 1.11 & 1.14 & 0.97 & 1.19 & 1.10 \\
\hline $\mathrm{TiO}_{2}$ & 1.48 & 1.56 & 1.32 & 1.78 & 1.76 & 1.48 & 1.75 & 1.64 & 1.75 \\
\hline $\mathrm{MnO}_{2}$ & 65.47 & 68.50 & 65.93 & 68.73 & 68.14 & 66.35 & 65.87 & 68.53 & 67.77 \\
\hline $\mathrm{CeO}_{2}$ & - & - & - & - & n/a & - & - & - & 0.37 \\
\hline $\mathrm{Al}_{2} \mathrm{O}_{3}$ & 0.49 & 0.47 & 0.47 & 0.55 & 0.65 & 0.56 & 0.55 & 0.52 & 0.64 \\
\hline $\mathrm{Fe}_{2} \mathrm{O}_{3}$ & 5.29 & 5.08 & 5.43 & 5.22 & 5.16 & 5.06 & 5.46 & 4.94 & 5.41 \\
\hline $\mathrm{MgO}$ & 0.30 & 0.10 & 0.23 & 0.26 & 0.07 & 0.27 & 0.06 & 0.21 & 0.11 \\
\hline $\mathrm{CaO}$ & 0.81 & 0.70 & 0.85 & 0.79 & 0.82 & 0.70 & 0.64 & 0.78 & 0.71 \\
\hline Zno & - & 0.26 & 0.38 & 0.37 & 0.20 & 0.37 & - & - & 0.21 \\
\hline SrO & 1.19 & 1.12 & 1.41 & 1.27 & 0.96 & 1.17 & 1.10 & 1.35 & 0.99 \\
\hline $\mathrm{BaO}$ & 6.69 & 6.96 & 7.00 & 7.12 & 7.21 & 7.30 & 7.37 & 7.80 & 8.11 \\
\hline $\mathrm{PbO}$ & $\mathrm{n} / \mathrm{a}$ & $\mathrm{n} / \mathrm{a}$ & $\mathrm{n} / \mathrm{a}$ & $\mathrm{n} / \mathrm{a}$ & 0.16 & $\mathrm{n} / \mathrm{a}$ & $\mathrm{n} / \mathrm{a}$ & $\mathrm{n} / \mathrm{a}$ & - \\
\hline $\mathrm{Na}_{2} \mathrm{O}$ & 0.72 & 0.74 & 0.89 & 0.72 & 0.70 & 0.68 & 0.65 & 0.65 & 0.69 \\
\hline $\mathrm{K}_{2} \mathrm{O}$ & 1.61 & 1.61 & 1.44 & 1.76 & 2.06 & 1.66 & 1.52 & 1.56 & 1.54 \\
\hline $\mathrm{La}_{2} \mathrm{O}_{3}$ & $\mathrm{n} / \mathrm{a}$ & n/a & $\mathrm{n} / \mathrm{a}$ & n/a & 0.19 & n/a & $\mathrm{n} / \mathrm{a}$ & $\mathrm{n} / \mathrm{a}$ & 0.14 \\
\hline Total & 85.11 & 88.12 & 86.47 & 89.59 & 89.19 & 86.74 & 85.94 & 89.17 & 89.54 \\
\hline $\mathrm{Si}^{+4}$ & 0.16 & 0.15 & 0.16 & 0.14 & 0.16 & 0.17 & 0.14 & 0.17 & 0.16 \\
\hline $\mathrm{Ti}^{+4}$ & 0.16 & 0.17 & 0.15 & 0.19 & 0.19 & 0.16 & 0.19 & 0.18 & 0.19 \\
\hline $\mathrm{Mn}^{+4}$ & 6.70 & 6.77 & 6.68 & 6.70 & 6.69 & 6.69 & 6.70 & 6.73 & 6.66 \\
\hline $\mathrm{Ce}^{+4}$ & - & - & - & - & $n / a$ & - & - & - & 0.02 \\
\hline $\mathrm{Al}^{+3}$ & 0.09 & 0.08 & 0.08 & 0.09 & 0.11 & 0.10 & 0.10 & 0.09 & 0.11 \\
\hline $\mathrm{Fe}^{+3}$ & 0.59 & 0.55 & 0.60 & 0.55 & 0.55 & 0.56 & 0.61 & 0.53 & 0.58 \\
\hline $\mathrm{Mg}^{+2}$ & 0.07 & 0.02 & 0.05 & 0.05 & 0.01 & 0.06 & 0.01 & 0.04 & 0.02 \\
\hline $\mathrm{Ca}^{+2}$ & 0.13 & 0.11 & 0.13 & 0.12 & 0.12 & 0.11 & 0.10 & 0.12 & 0.11 \\
\hline $\mathrm{Zn}^{+2}$ & - & 0.03 & 0.04 & 0.04 & 0.02 & 0.04 & - & - & 0.02 \\
\hline $\mathrm{Sr}^{+2}$ & 0.10 & 0.09 & 0.12 & 0.10 & 0.08 & 0.10 & 0.09 & 0.11 & 0.08 \\
\hline $\mathrm{Ba}^{+2}$ & 0.39 & 0.39 & 0.40 & 0.39 & 0.40 & 0.42 & 0.43 & 0.43 & 0.45 \\
\hline $\mathrm{Pb}^{+2}$ & $n / a$ & $n / a$ & $n / a$ & $n / a$ & 0.01 & $n / a$ & n/a & n/a & - \\
\hline $\mathrm{Na}^{+1}$ & 0.21 & 0.21 & 0.25 & 0.20 & 0.19 & 0.19 & 0.19 & 0.18 & 0.19 \\
\hline $\mathrm{K}^{+1}$ & 0.30 & 0.29 & 0.27 & 0.32 & 0.37 & 0.31 & 0.29 & 0.28 & 0.28 \\
\hline $\mathrm{La}^{+3}$ & $\mathrm{n} / \mathrm{a}$ & $\mathrm{n} / \mathrm{a}$ & $\mathrm{n} / \mathrm{a}$ & $\mathrm{n} / \mathrm{a}$ & 0.01 & $\mathrm{n} / \mathrm{a}$ & $n / a$ & $\mathrm{n} / \mathrm{a}$ & 0.01 \\
\hline Total & 8.89 & 8.85 & 8.93 & 8.90 & 8.92 & 8.90 & 8.85 & 8.85 & 8.87 \\
\hline $\mathrm{O}^{-2}$ & 16.00 & 16.00 & 16.00 & 16.00 & 16.00 & 16.00 & 16.00 & 16.00 & 16.00 \\
\hline
\end{tabular}

analyzed for but not detected: $\mathrm{Cl}, \mathrm{Y}, \mathrm{Ni}$, and $\mathrm{P}$ 
Cryptomelane-Hollandite

\begin{tabular}{|c|c|c|c|c|c|c|c|c|c|c|c|c|c|}
\hline $\begin{array}{l}\mathrm{SiO}_{2} \\
\mathrm{TiO}_{2} \\
\mathrm{MnO}_{2}\end{array}$ & $\begin{array}{r}0.46 \\
0.38 \\
81.68\end{array}$ & $\begin{array}{r}0.56 \\
0.39 \\
81.15\end{array}$ & $\begin{array}{r}0.36 \\
0.17 \\
83.25\end{array}$ & $\begin{array}{r}0.57 \\
0.52 \\
75.26\end{array}$ & $\begin{array}{r}0.81 \\
0.73 \\
69.43\end{array}$ & $\begin{array}{r}0.42 \\
0.24 \\
83.50\end{array}$ & $\begin{array}{r}0.79 \\
0.44 \\
78.62\end{array}$ & $\begin{array}{r}0.58 \\
0.36 \\
83.15\end{array}$ & $\begin{array}{r}0.36 \\
0.17 \\
83.46\end{array}$ & $\begin{array}{r}0.55 \\
0.40 \\
82.01\end{array}$ & $\begin{array}{r}0.47 \\
0.32 \\
80.58\end{array}$ & $\begin{array}{r}0.42 \\
0.15 \\
80.31\end{array}$ & $\begin{array}{r}0.50 \\
0.31 \\
82.68\end{array}$ \\
\hline $\mathrm{CeO}_{2}$ & - & n/a & & $\mathrm{n} / \mathrm{a}$ & $\mathrm{n} / \mathrm{a}$ & & $\mathrm{n} / \mathrm{a}$ & & $\mathrm{n} / \mathrm{a}$ & & 0.60 & - & - \\
\hline $\mathrm{I}_{2} \mathrm{O}_{3}$ & 0.48 & 0.49 & 0.3 & 0.87 & 0.86 & 0.32 & 0.60 & 0.52 & 0.40 & 0.26 & 0.36 & 0.35 & 0.38 \\
\hline $\mathrm{e}_{2} \mathrm{O}_{3}$ & 1.98 & 2.28 & 1.30 & 2.56 & 3.33 & 2.15 & 3.00 & 2.86 & 1.65 & 2.31 & 2.27 & 1.97 & 2.10 \\
\hline $\mathrm{MgO}$ & 0.08 & 0.10 & 0.09 & 0.05 & 0.12 & - & 0.13 & 0.13 & 0.08 & 0.08 & 0.16 & 0.22 & 0.10 \\
\hline $\mathrm{CaO}$ & 0.48 & 0.54 & 0.44 & 0.43 & 0.63 & 0.63 & 0.65 & 0.59 & 0.52 & 0.71 & 0.71 & 0.62 & 0.63 \\
\hline $\mathrm{NiO}$ & - & - & & - & & & - & . & - & - & - & - & - \\
\hline $\mathrm{ZnO}$ & 0.17 & 0.18 & 0.22 & - & - & 0.22 & - & 0.22 & - & - & - & - & - \\
\hline $\mathrm{SrO}$ & 0.84 & 0.80 & 0.88 & 0.56 & 0.71 & 1.04 & 0.93 & 1.22 & 0.88 & 1.13 & 1.17 & 1.17 & 0.97 \\
\hline $\mathrm{BaO}$ & 3.76 & 4.50 & 4.52 & 4.78 & 4.90 & 4.98 & 5.03 & 5.09 & 5.14 & 5.17 & 5.26 & 5.37 & 5.38 \\
\hline $\mathrm{PbO}$ & - & 0.17 & - & 0.13 & - & $\mathrm{n} / \mathrm{a}$ & - & $\mathrm{n} / \mathrm{a}$ & - & $\mathrm{n} / \mathrm{a}$ & $\mathrm{n} / \mathrm{a}$ & $\mathrm{n} / \mathrm{a}$ & $\mathrm{n} / \mathrm{a}$ \\
\hline $\mathrm{Na}_{2} \mathrm{O}$ & 0.29 & 0.37 & 0.31 & 0.27 & 0.26 & 0.39 & 0.34 & 0.39 & 0.36 & 0.42 & 0.42 & 0.45 & 0.48 \\
\hline $\mathrm{K}_{2} \mathrm{O}$ & 3.92 & 3.83 & 4.45 & 4.08 & 3.44 & 3.61 & 3.49 & 3.43 & 3.66 & 3.22 & 3.61 & 3.59 & 3.57 \\
\hline $\mathrm{La}_{2} \mathrm{O}_{3}$ & - & 0.14 & - & - & 0.09 & $\mathrm{n} / \mathrm{a}$ & - & n/a & - & $\mathrm{n} / \mathrm{a}$ & $\mathrm{n} / \mathrm{a}$ & $\mathrm{n} / \mathrm{a}$ & $\mathrm{n} / \mathrm{a}$ \\
\hline $\mathrm{P}_{2} \mathrm{O}_{5}$ & 0.06 & 0.10 & 0.09 & 0.07 & 0.13 & $\mathrm{n} / \mathrm{a}$ & - & n/a & 0.08 & $\mathrm{n} / \mathrm{a}$ & $\mathrm{n} / \mathrm{a}$ & $\mathrm{n} / \mathrm{a}$ & $\mathrm{n} / \mathrm{a}$ \\
\hline Total & 94.58 & 95.60 & 96.47 & 90.15 & 85.44 & 97.50 & 94.02 & 98.54 & 96.76 & 96.26 & 95.33 & 94.62 & 97.10 \\
\hline $\mathrm{Si}^{+4}$ & 0.06 & 0.07 & 0.05 & 0.08 & 0.12 & 0.05 & 0.10 & 0.07 & 0.05 & 0.0 & 0.06 & 0.06 & 0.06 \\
\hline $\mathrm{Ti}^{+4}$ & 0.04 & 0.04 & 0.02 & 0.05 & 0.08 & 0.02 & 0.04 & 0.03 & 0.02 & 0.04 & 0.03 & 0.01 & 0.03 \\
\hline $\mathrm{Mn}^{+4}$ & 7.34 & 7.26 & 7.39 & 7.16 & 6.98 & 7.35 & 7.17 & 7.24 & 7.39 & 7.30 & 7.28 & 7.31 & 7.31 \\
\hline $\mathrm{Ce}^{+4}$ & - & n/a & $\mathrm{n} / \mathrm{a}$ & $n / a$ & $\mathrm{n} / \mathrm{a}$ & - & $\mathrm{n} / \mathrm{a}$ & & $\mathrm{n} / \mathrm{a}$ & - & - & - & - \\
\hline $\mathrm{Al}^{+3}$ & 0.07 & 0.07 & 0.06 & 0.14 & 0.15 & 0.05 & 0.09 & 0.08 & 0.06 & 0.04 & 0.06 & 0.05 & 0.06 \\
\hline $\mathrm{Fe}^{+3}$ & 0.19 & 0.22 & 0.13 & 0.27 & 0.36 & 0.21 & 0.30 & 0.27 & 0.16 & 0.22 & 0.22 & 0.20 & 0.20 \\
\hline $\mathrm{Mg}^{+2}$ & 0.02 & 0.02 & 0.02 & 0.01 & 0.03 & - & 0.03 & 0.02 & 0.02 & 0.02 & 0.03 & 0.04 & 0.02 \\
\hline $\mathrm{Ca}^{+2}$ & 0.07 & 0.07 & 0.06 & 0.06 & 0.10 & 0.09 & 0.09 & 0.08 & 0.07 & 0.10 & 0.10 & 0.09 & 0.09 \\
\hline $\mathrm{Ni}^{+2}$ & - & - & & - & - & - & - & - & - & - & - & - & - \\
\hline $\mathrm{Zn}^{+2}$ & 0.02 & 0.02 & 0.02 & - & - & 0.02 & - & 0.02 & - & - & - & - & - \\
\hline $\mathrm{Sr}^{+2}$ & 0.06 & 0.06 & 0.07 & 0.04 & 0.06 & 0.08 & 0.07 & 0.09 & 0.07 & 0.08 & 0.09 & 0.09 & 0.07 \\
\hline $\mathrm{Ba}^{+2}$ & 0.19 & 0.23 & 0.23 & 0.26 & 0.28 & 0.25 & 0.26 & 0.25 & 0.26 & 0.26 & 0.27 & 0.28 & 0.27 \\
\hline $\mathrm{Pb}^{+2}$ & . & 0.01 & - & 0.00 & - & $\mathrm{n} / \mathrm{a}$ & - & $\mathrm{n} / \mathrm{a}$ & - & $\mathrm{n} / \mathrm{a}$ & $\mathrm{n} / \mathrm{a}$ & $\mathrm{n} / \mathrm{a}$ & $\mathrm{n} / \mathrm{a}$ \\
\hline $\mathrm{Na}^{+1}$ & 0.07 & 0.09 & 0.08 & 0.07 & 0.07 & 0.10 & 0.09 & 0.10 & 0.09 & 0.10 & 0.11 & 0.11 & 0.12 \\
\hline $\mathrm{K}^{+1}$ & 0.65 & 0.63 & 0.73 & 0.72 & 0.64 & 0.59 & 0.59 & 0.55 & 0.60 & 0.53 & 0.60 & 0.60 & 0.58 \\
\hline $\mathrm{La}^{+3}$ & & 0.01 & - & - & 0.00 & $\mathrm{n} / \mathrm{a}$ & - & $\mathrm{n} / \mathrm{a}$ & - & $\mathrm{n} / \mathrm{a}$ & $\mathrm{n} / \mathrm{a}$ & $\mathrm{n} / \mathrm{a}$ & $\mathrm{n} / \mathrm{a}$ \\
\hline & 0.01 & 0.01 & 0.01 & 0.01 & 0.02 & $\mathrm{n} / \mathrm{a}$ & - & $\mathrm{n} / \mathrm{a}$ & 0.01 & $n / a$ & $\mathrm{n} / \mathrm{a}$ & $\mathrm{n} / \mathrm{a}$ & $\mathrm{n} / \mathrm{a}$ \\
\hline & 8.78 & 8.82 & 8.84 & 8.88 & 8.89 & 8.79 & 8.83 & 8.80 & 8.77 & 8.77 & 8.85 & 8.85 & 8.81 \\
\hline & 16.00 & 16.00 & 16.00 & 16.00 & 16.00 & 16.00 & 16.00 & 16.00 & 16.00 & 16.00 & 16.00 & 16.00 & 16.00 \\
\hline
\end{tabular}


Cryptomelane-Hollandite

\begin{tabular}{|c|c|c|c|c|c|c|c|c|c|c|c|c|c|}
\hline $\mathrm{SiO}_{2}$ & 0.52 & 0.47 & 0.53 & 0.34 & 0.45 & 0.50 & 0.56 & 1.03 & 0.52 & 0.54 & 0.52 & 0.94 & 1.05 \\
\hline $\mathrm{TiO}_{2}$ & 0.35 & 0.39 & 0.24 & 0.11 & 0.18 & 0.12 & 0.21 & 0.79 & 0.26 & 0.21 & 0.28 & 1.06 & 0.79 \\
\hline $\operatorname{lnO}_{2}$ & 80.20 & 82.02 & 82.20 & 83.10 & 81.98 & 80.93 & 79.14 & 68.20 & 81.54 & 81.42 & 81.35 & 72.95 & 68.61 \\
\hline $\mathrm{eO}_{2}$ & 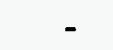 & - & $\mathrm{n} / \mathrm{a}$ & - & $n / a$ & $\mathrm{n} / \mathrm{a}$ & $\mathrm{n} / \mathrm{a}$ & $\mathrm{n} / \mathrm{a}$ & 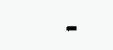 & n/a & n/a & - & n/a \\
\hline $\mathrm{I}_{2} \mathrm{O}_{3}$ & 0.38 & 0.42 & 0.40 & 0.33 & 0.43 & 0.39 & 0.36 & 0.75 & 0.38 & 0.37 & 0.53 & 0.65 & 0.74 \\
\hline $\mathrm{e}_{2} \mathrm{O}_{3}$ & 2.51 & 2.40 & 1.99 & 1.45 & 2.03 & 1.99 & 2.39 & 4.50 & 1.93 & 2.00 & 2.26 & 4.49 & 4.36 \\
\hline go & 0.15 & 0.09 & 0.10 & 0.06 & 0.13 & 0.07 & 0.12 & 0.10 & 0.10 & 0.10 & 0.18 & 0.20 & 0.13 \\
\hline $\mathrm{aO}$ & 0.65 & 0.73 & 0.64 & 0.56 & 0.56 & 0.58 & 0.69 & 0.72 & 0.61 & 0.61 & 0.70 & 0.94 & 0.81 \\
\hline $\mathrm{liO}$ & - & - & - & - & - & & - & & - & - & - & - & \\
\hline no & - & - & - & - & 0.24 & - & 0.18 & - & - & - & - & - & 0.19 \\
\hline 0 & 0.96 & 1.18 & 0.81 & 1.21 & 0.81 & 0.78 & 0.91 & 0.85 & 1.18 & 0.78 & 1.00 & 0.84 & 0.78 \\
\hline $\mathrm{aO}$ & 5.39 & 5.40 & 5.46 & 5.47 & 5.51 & 5.54 & 5.62 & 5.63 & 5.63 & 5.65 & 5.65 & 5.66 & 5.68 \\
\hline & - & $\mathrm{n} / \mathrm{a}$ & - & $n / a$ & 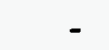 & 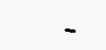 & 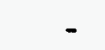 & - & $\mathrm{n} / \mathrm{a}$ & - & - & $\mathrm{n} / \mathrm{a}$ & \\
\hline $\mathrm{a}_{2} \mathrm{O}$ & 0.45 & 0.42 & 0.33 & 0.38 & 0.37 & 0.39 & 0.39 & 0.31 & 0.47 & 0.34 & 0.51 & 0.40 & 0.28 \\
\hline & 2.97 & 3.49 & 3.63 & 3.69 & 3.60 & 3.67 & 3.19 & 2.44 & 3.54 & 3.20 & 2.71 & 2.47 & 2.70 \\
\hline $\mathrm{a}_{2} \mathrm{O}_{3}$ & 0.14 & $\mathrm{n} / \mathrm{a}$ & 0.16 & $\mathrm{n} / \mathrm{a}$ & - & - & 0.12 & 0.15 & $n / a$ & 0.10 & 0.11 & $\mathrm{n} / \mathrm{a}$ & 0.10 \\
\hline & 0.17 & $\mathrm{n} / \mathrm{a}$ & 0.16 & $n / a$ & 0.13 & 0.12 & 0.15 & - & $\mathrm{n} / \mathrm{a}$ & 0.16 & - & $n / a$ & 0.22 \\
\hline tal & 94.84 & 97.01 & 96.65 & 96.70 & 96.42 & 95.08 & 94.03 & 85.47 & 96.16 & 95.48 & 95.80 & 90.60 & 86.44 \\
\hline & 0.07 & 0.06 & 0.07 & 0.04 & 0.06 & 0.07 & 0.07 & 0.15 & 0.07 & 0.07 & 0.07 & 0.13 & 0.15 \\
\hline $\mathrm{Ti}^{+4}$ & 0.03 & 0.04 & 0.02 & 0.01 & 0.02 & 0.01 & 0.02 & 0.09 & 0.03 & 0.02 & 0.03 & 0.11 & 0.09 \\
\hline $\mathrm{Mn}^{+4}$ & 7.25 & 7.27 & 7.30 & 7.40 & 7.31 & 7.32 & 7.25 & 6.89 & 7.31 & 7.31 & 7.28 & 6.92 & 6.85 \\
\hline $\mathrm{Ce}^{+4}$ & - & - & $\mathrm{n} / \mathrm{a}$ & - & $\mathrm{n} / \mathrm{a}$ & $n / a$ & $\mathrm{n} / \mathrm{a}$ & $\mathrm{n} / \mathrm{a}$ & - & $\mathrm{n} / \mathrm{a}$ & $\mathrm{n} / \mathrm{a}$ & - & $n / a$ \\
\hline & 0.06 & 0.06 & 0.06 & 0.05 & 0.07 & 0.06 & 0.06 & 0.13 & 0.06 & 0.06 & 0.08 & 0.11 & 0.13 \\
\hline $\mathrm{Fe}^{+3}$ & 0.25 & 0.23 & 0.19 & 0.14 & 0.20 & 0.20 & 0.24 & 0.49 & 0.19 & 0.20 & 0.22 & 0.46 & 0.47 \\
\hline $\mathrm{Mg}^{+2}$ & 0.03 & 0.02 & 0.02 & 0.01 & 0.02 & 0.01 & 0.02 & 0.02 & 0.02 & 0.02 & 0.03 & 0.04 & 0.03 \\
\hline $\mathrm{Ca}^{+2}$ & 0.09 & 0.10 & 0.09 & 0.08 & 0.08 & 0.08 & 0.10 & 0.11 & 0.08 & 0.08 & 0.10 & 0.14 & 0.13 \\
\hline $\mathrm{Ni}^{+2}$ & - & - & - & - & - & - & - & - & - & - & - & - & - \\
\hline $\mathrm{Zn}^{+2}$ & - & - & - & - & 0.02 & - & 0.02 & - & - & - & - & - & 0.02 \\
\hline $\mathrm{Sr}^{+2}$ & 0.07 & 0.09 & 0.06 & 0.09 & 0.06 & 0.06 & 0.07 & 0.07 & 0.09 & 0.06 & 0.08 & 0.07 & 0.07 \\
\hline $\mathrm{Ba}^{+2}$ & 0.28 & 0.27 & 0.27 & 0.28 & 0.28 & 0.28 & 0.29 & 0.32 & 0.29 & 0.29 & 0.29 & 0.30 & 0.32 \\
\hline $\mathrm{b}^{+2}$ & - & $\mathrm{n} / \mathrm{a}$ & . & $\mathrm{n} / \mathrm{a}$ & - & - & - & - & n/a & - & - & $\mathrm{n} / \mathrm{a}$ & - \\
\hline $\mathrm{Va}^{+1}$ & 0.11 & 0.10 & 0.08 & 0.09 & 0.09 & 0.10 & 0.10 & 0.09 & 0.12 & 0.09 & 0.13 & 0.11 & 0.08 \\
\hline+ & 0.50 & 0.57 & 0.60 & 0.61 & 0.59 & 0.61 & 0.54 & 0.45 & 0.59 & 0.53 & 0.45 & 0.43 & 0.50 \\
\hline $\mathrm{La}^{+3}$ & 0.01 & $\mathrm{n} / \mathrm{a}$ & 0.01 & $\mathrm{n} / \mathrm{a}$ & - & - & 0.01 & 0.01 & $\mathrm{n} / \mathrm{a}$ & 0.00 & 0.01 & $\mathrm{n} / \mathrm{a}$ & 0.01 \\
\hline & 0.02 & n/a & 0.02 & $\mathrm{n} / \mathrm{a}$ & 0.01 & 0.01 & 0.02 & - & $\mathrm{n} / \mathrm{a}$ & 0.02 & - & $\mathrm{n} / \mathrm{a}$ & 0.03 \\
\hline & 8.77 & 8.82 & 8.79 & 8.80 & 8.81 & 8.81 & 8.80 & 8.83 & 8.83 & 8.75 & 8.76 & 8.82 & 8.86 \\
\hline & 16.00 & 16.00 & 16.00 & 16.00 & 16.00 & 16.00 & 16.00 & 16.00 & 16.00 & 16.00 & 16.00 & 16.00 & 16.00 \\
\hline
\end{tabular}


Manganese Oxides from USW G-4 2947, continued

Cryptomelane-Hollandite

\begin{tabular}{|c|c|c|c|c|c|c|c|c|c|c|c|c|}
\hline $\mathrm{SiO}_{2}$ & 0.59 & 0.95 & 0.57 & 0.74 & 0.53 & 0.51 & 0.97 & 0.59 & 0.89 & 0.53 & 0.55 & 0.56 \\
\hline $\mathrm{TiO}_{2}$ & 0.43 & 0.85 & 0.24 & 0.31 & 0.27 & 0.34 & 0.83 & 0.22 & 0.29 & 0.32 & 0.48 & 0.64 \\
\hline $\mathrm{MnO}_{2}$ & 77.27 & 71.23 & 79.64 & 80.41 & 76.34 & 79.04 & 71.99 & 79.34 & 75.62 & 77.00 & 77.06 & 77.73 \\
\hline $\mathrm{CeO}_{2}$ & $\mathrm{n} / \mathrm{a}$ & 0.19 & $\mathrm{n} / \mathrm{a}$ & - & 0.27 & - & n/a & n/a & n/a & - & - & - \\
\hline $\mathrm{Al}_{2} \mathrm{O}_{3}$ & 0.65 & 0.67 & 0.41 & 0.42 & 0.44 & 0.37 & 0.47 & 0.43 & 0.63 & 0.72 & 0.60 & 0.54 \\
\hline $\mathrm{Fe}_{2} \mathrm{O}_{3}$ & 2.59 & 4.03 & 2.36 & 2.67 & 2.27 & 2.43 & 5.77 & 2.26 & 3.61 & 1.64 & 1.94 & 2.44 \\
\hline $\mathrm{MgO}$ & 0.11 & 0.37 & 0.12 & 0.19 & 0.13 & 0.10 & 0.58 & 0.13 & 0.26 & 0.15 & 0.21 & 0.25 \\
\hline $\mathrm{CaO}$ & 0.63 & 0.85 & 0.62 & 0.72 & 0.63 & 0.78 & 1.40 & 0.68 & 1.34 & 1.09 & 1.16 & 1.26 \\
\hline $\mathrm{NiO}$ & - & - & - & - & 0.20 & - & - & - & - & - & - & 0.12 \\
\hline $\mathrm{ZnO}$ & 0.15 & - & - & 0.30 & - & - & 0.20 & 0.28 & 0.21 & - & - & 0.41 \\
\hline SrO & 0.78 & 0.66 & 0.84 & 1.32 & 0.92 & 1.27 & 1.08 & 0.92 & 1.14 & 1.19 & 1.21 & 1.61 \\
\hline $\mathrm{BaO}$ & 5.81 & 5.85 & 6.10 & 6.12 & 6.20 & 6.21 & 6.35 & 6.50 & 6.58 & 8.32 & 9.60 & 9.75 \\
\hline $\mathrm{PbO}$ & - & - & - & $\mathrm{n} / \mathrm{a}$ & - & $\mathrm{n} / \mathrm{a}$ & - & - & - & $\mathrm{n} / \mathrm{a}$ & $\mathrm{n} / \mathrm{a}$ & $\mathrm{n} / \mathrm{a}$ \\
\hline $\mathrm{Na}_{2} \mathrm{O}$ & 0.26 & 0.34 & 0.34 & 0.40 & 0.33 & 0.42 & 0.46 & 0.38 & 0.79 & 0.47 & 0.58 & 0.64 \\
\hline $\mathrm{K}_{2} \mathrm{O}$ & 3.08 & 1.86 & 3.25 & 2.79 & 1.89 & 3.02 & 1.23 & 2.77 & 0.49 & 0.48 & 0.47 & 0.49 \\
\hline $\mathrm{La}_{2} \mathrm{O}_{3}$ & 0.14 & - & - & $\mathrm{n} / \mathrm{a}$ & 0.15 & $\mathrm{n} / \mathrm{a}$ & 0.11 & 0.09 & 0.22 & $\mathrm{n} / \mathrm{a}$ & $\mathrm{n} / \mathrm{a}$ & $\mathrm{n} / \mathrm{a}$ \\
\hline $\mathrm{P}_{2} \mathrm{O}_{5}$ & - & 0.19 & 0.09 & $n / a$ & 0.14 & $\mathrm{n} / \mathrm{a}$ & 0.09 & 0.20 & - & $\mathrm{n} / \mathrm{a}$ & $\mathrm{n} / \mathrm{a}$ & n/a \\
\hline Total & 92.49 & 88.04 & 94.58 & 96.39 & 90.71 & 94.49 & 91.53 & 94.79 & 92.07 & 91.91 & 93.86 & 96.44 \\
\hline $\mathrm{Si}^{+4}$ & 0.08 & 0.13 & 0.08 & 0.10 & 0.07 & 0.07 & 0.13 & 0.08 & 0.12 & 0.07 & 0.07 & 0.07 \\
\hline $\mathrm{Ti}^{+4}$ & 0.04 & 0.09 & 0.02 & 0.03 & 0.03 & 0.03 & 0.09 & 0.02 & 0.03 & 0.03 & 0.05 & 0.06 \\
\hline $\mathrm{Mn}^{+4}$ & 7.20 & 6.94 & 7.26 & 7.20 & 7.25 & 7.24 & 6.80 & 7.23 & 7.07 & 7.26 & 7.19 & 7.10 \\
\hline $\mathrm{Ce}^{+4}$ & $\mathrm{n} / \mathrm{a}$ & 0.01 & $\mathrm{n} / \mathrm{a}$ & - & 0.01 & - & $\mathrm{n} / \mathrm{a}$ & n/a & $\mathrm{n} / \mathrm{a}$ & - & - & - \\
\hline $\mathrm{Al}^{+3}$ & 0.10 & 0.11 & 0.06 & 0.06 & 0.07 & 0.06 & 0.08 & 0.07 & 0.10 & 0.12 & 0.10 & 0.08 \\
\hline $\mathrm{Fe}^{+3}$ & 0.26 & 0.43 & 0.23 & 0.26 & 0.23 & 0.24 & 0.59 & 0.22 & 0.37 & 0.17 & 0.20 & 0.24 \\
\hline $\mathrm{Mg}^{+2}$ & 0.02 & 0.08 & 0.02 & 0.04 & 0.03 & 0.02 & 0.12 & 0.03 & 0.05 & 0.03 & 0.04 & 0.05 \\
\hline $\mathrm{Ca}^{+2}$ & 0.09 & 0.13 & 0.09 & 0.10 & 0.09 & 0.11 & 0.20 & 0.10 & 0.19 & 0.16 & 0.17 & 0.18 \\
\hline $\mathrm{Ni}^{+2}$ & - & - & - & - & 0.02 & - & - & - & - & - & - & 0.01 \\
\hline $\mathrm{Zn}^{+2}$ & 0.01 & - & - & 0.03 & - & - & 0.02 & 0.03 & 0.02 & - & - & 0.04 \\
\hline $\mathrm{Sr}^{+2}$ & 0.06 & 0.05 & 0.06 & 0.10 & 0.07 & 0.10 & 0.09 & 0.07 & 0.09 & 0.09 & 0.09 & 0.12 \\
\hline $\mathrm{Ba}^{+2}$ & 0.31 & 0.32 & 0.32 & 0.31 & 0.33 & 0.32 & 0.34 & 0.34 & 0.35 & 0.45 & 0.51 & 0.51 \\
\hline $\mathrm{Pb}^{+2}$ & - & - & - & $\mathrm{n} / \mathrm{a}$ & - & $\mathrm{n} / \mathrm{a}$ & - & - & - & $\mathrm{n} / \mathrm{a}$ & $\mathrm{n} / \mathrm{a}$ & $\mathrm{n} / \mathrm{a}$ \\
\hline $\mathrm{Na}^{+1}$ & 0.07 & 0.09 & 0.09 & 0.10 & 0.09 & 0.11 & 0.12 & 0.10 & 0.21 & 0.12 & 0.15 & 0.16 \\
\hline $\mathbf{K}^{+1}$ & 0.53 & 0.33 & 0.55 & 0.46 & 0.33 & 0.51 & 0.21 & 0.47 & 0.08 & 0.08 & 0.08 & 0.08 \\
\hline $\mathrm{La}^{+3}$ & 0.01 & - & - & $\mathrm{n} / \mathrm{a}$ & 0.01 & $\mathrm{n} / \mathrm{a}$ & 0.01 & 0.00 & 0.01 & $\mathrm{n} / \mathrm{a}$ & $\mathrm{n} / \mathrm{a}$ & $\mathrm{n} / \mathrm{a}$ \\
\hline $\mathrm{P}^{+5}$ & - & 0.02 & 0.01 & $\mathrm{n} / \mathrm{a}$ & 0.02 & $\mathrm{n} / \mathrm{a}$ & 0.01 & 0.02 & - & $\mathrm{n} / \mathrm{a}$ & $\mathrm{n} / \mathrm{a}$ & $\mathrm{n} / \mathrm{a}$ \\
\hline Total & 8.79 & 8.74 & 8.79 & 8.79 & 8.66 & 8.81 & 8.80 & 8.77 & 8.69 & 8.59 & 8.65 & 8.72 \\
\hline $\mathrm{O}^{-2}$ & 16.00 & 16.00 & 16.00 & 16.00 & 16.00 & 16.00 & 16.00 & 16.00 & 16.00 & 16.00 & 16.00 & 16.00 \\
\hline
\end{tabular}

${ }^{\text {a }}$ Analyzed for but not detected: $\mathrm{Cl}$ and $\mathrm{Y}$ 
Cryptomelane-Hollandite $+/$ - Todorokite

\begin{tabular}{|c|c|c|c|c|c|c|c|c|c|c|c|c|}
\hline $\mathrm{SiO}_{2}$ & 0.09 & 0.32 & 0.18 & 0.28 & 0.14 & 0.30 & 0.31 & 0.17 & 0.29 & 0.49 & 0.34 & 0.20 \\
\hline $\mathrm{iO}_{2}$ & - & 0.45 & 0.74 & 0.35 & 0.15 & 0.41 & 0.62 & 0.17 & 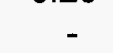 & 0.22 & 0.51 & 0.21 \\
\hline $\mathrm{InO}_{2}$ & 76.31 & 82.77 & 77.91 & 79.26 & 78.14 & 75.31 & 78.79 & 80.96 & 76.46 & 76.94 & 77.96 & 78.69 \\
\hline $\mathrm{eO}_{2}$ & $\mathrm{n} / \mathrm{a}$ & - & - & $\mathrm{n} / \mathrm{a}$ & & $\mathrm{n} / \mathrm{a}$ & $\mathrm{n} / \mathrm{a}$ & 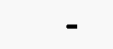 & 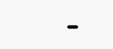 & & $n / a$ & \\
\hline $\mathrm{H}_{2} \mathrm{O}_{3}$ & 0.11 & 0.44 & 0.13 & 0.60 & 0.18 & 0.41 & 0.93 & 0.17 & 0.15 & 0.22 & & 0.14 \\
\hline $\mathrm{e}_{2} \mathrm{O}_{3}$ & 1.33 & 2.94 & 1.89 & 2.24 & 1.80 & 2.55 & 3.32 & 1.69 & 2.55 & 4.60 & 2.63 & 1.96 \\
\hline $1 \mathrm{gO}$ & 2.69 & 0.22 & 2.23 & 0.66 & 2.37 & 0.41 & 0.3 & 1.7 & 1.49 & 0.78 & 0. & 2.18 \\
\hline $\mathrm{aO}$ & 1.20 & 0.59 & 1.08 & 0.77 & 1.21 & 0.74 & 0.5 & 1.1 & 1.23 & 1.42 & 0.67 & 1.20 \\
\hline no & 0.15 & - & - & - & 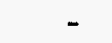 & - & - & 0.2 & - & 0.2 & & - \\
\hline $\mathrm{rO}$ & 0.95 & 1.48 & 1.18 & 1.04 & 0.97 & 1.34 & 1.27 & 1. & 1.38 & 2. & 1. & 1.15 \\
\hline $3 \mathrm{OO}$ & 3.04 & 3.70 & 3.70 & 3.85 & 3.87 & 4.0 & $4 . C$ & 4.0 & 4.05 & 4.0 & 4.21 & 4.22 \\
\hline bo & - & $\mathrm{n} / \mathrm{a}$ & $n / a$ & - & $n / a$ & 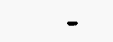 & - & $\mathrm{n} / \mathrm{a}$ & $\mathrm{n} / \mathrm{a}$ & $\mathrm{n} / \mathrm{a}$ & - & $\mathrm{n} / \mathrm{a}$ \\
\hline $\mathrm{Na}_{2} \mathrm{O}$ & 1.55 & 0.75 & 1.50 & 1.14 & 1.43 & 0.91 & 0.75 & 1.33 & 1.18 & 1.23 & 0.64 & 1.51 \\
\hline $\mathrm{K}_{2} \mathrm{O}$ & 0.18 & 3.46 & 0.58 & 2.93 & 0.40 & 2.38 & 3.04 & 0.78 & 0.67 & 1.02 & 2.82 & 0.40 \\
\hline $\mathrm{La}_{2} \mathrm{O}_{3}$ & - & $\mathrm{n} / \mathrm{a}$ & $\mathrm{n} / \mathrm{a}$ & - & n/a & - & - & $\mathrm{n} / \mathrm{a}$ & $\mathrm{n} / \mathrm{a}$ & n/a & - & n/a \\
\hline Total & 87.60 & 97.12 & 91.12 & 93.12 & 90.66 & 88.79 & 93.96 & 93.84 & 89.45 & 93.30 & 91.75 & 91.8 \\
\hline $\mathrm{Si}^{+4}$ & 0.01 & 0.04 & 0.02 & 0.04 & 0.02 & 0.04 & 0.04 & 0.02 & 0.04 & & 0.0 & 0.03 \\
\hline $\mathrm{Ti}^{+4}$ & - & 0.04 & 0.07 & 0.03 & 0.02 & 0.04 & 0.06 & 0.0 & - & & & 0.02 \\
\hline $\mathrm{Mn}^{+4}$ & 7.27 & 7.26 & 7.18 & 7.24 & 7.24 & 7.24 & 7.15 & 7.28 & 7.23 & 7.05 & 7.24 & 7.22 \\
\hline $\mathrm{Ce}^{+4}$ & $\mathrm{n} / \mathrm{a}$ & - & - & $n / a$ & - & $\mathrm{n} / \mathrm{a}$ & $n / a$ & - & - & - & $\mathrm{n} / \mathrm{a}$ & \\
\hline $\mathrm{Al}^{+3}$ & 0.02 & 0.07 & 0.02 & 0.09 & 0.03 & 0.07 & 0.14 & 0.03 & 0.02 & 0.03 & 0.09 & 0.02 \\
\hline $\mathrm{Fe}^{+3}$ & 0.14 & 0.28 & 0.19 & 0.22 & 0.18 & 0.27 & 0.33 & 0.1 & 0.26 & 0.4 & 27 & 0.2 \\
\hline $\mathrm{Mg}^{+2}$ & 0.55 & 0.04 & 0.44 & 0.13 & 0.47 & 0.0 & 0.06 & 0.34 & 0.30 & 0.15 & 07 & 0.43 \\
\hline $\mathrm{Ca}^{+2}$ & 0.18 & 0.08 & 0.15 & 0.11 & 0.17 & 0.11 & 0.08 & 0.16 & 0.18 & 0.20 & 0.10 & 0.17 \\
\hline $\mathrm{Zn}^{+2}$ & 0.02 & - & - & - & - & - & - & 0.02 & - & 0.02 & - & \\
\hline $\mathrm{Sr}^{+2}$ & 0.08 & 0.11 & 0.09 & 0.08 & 0.08 & 0.11 & 0.10 & 0.11 & 0.11 & 0.16 & 0.08 & 0.0 \\
\hline $\mathrm{Ba}^{+2}$ & 0.16 & 0.18 & 0.19 & 0.20 & 0.20 & 0.22 & 0.21 & 0.21 & 0.22 & 0.21 & 0.22 & 0.2 \\
\hline $\mathrm{Pb}^{+2}$ & - & $\mathrm{n} / \mathrm{a}$ & $\mathrm{n} / \mathrm{a}$ & - & $\mathrm{n} / \mathrm{a}$ & - & - & $\mathrm{n} / \mathrm{a}$ & $\mathrm{n} / \mathrm{a}$ & $\mathrm{n} / \mathrm{a}$ & - & $\mathrm{n} / \mathrm{a}$ \\
\hline $\mathrm{Na}^{+1}$ & 0.41 & 0.18 & 0.39 & 0.29 & 0.37 & 0.25 & 0.19 & 0.34 & 0.31 & 0.32 & 0.17 & 0.3 \\
\hline $\mathrm{K}^{+1}$ & 0.03 & 0.56 & 0.10 & 0.49 & 0.07 & 0.42 & 0.51 & 0.13 & 0.12 & 0.17 & 0.48 & 0.0 \\
\hline $\mathrm{La}^{+3}$ & - & n/a & $\mathrm{n} / \mathrm{a}$ & - & $\mathrm{n} / \mathrm{a}$ & - & - & $\mathrm{n} / \mathrm{a}$ & $\mathrm{n} / \mathrm{a}$ & $\mathrm{n} / \mathrm{a}$ & - & $\mathrm{n} / \mathrm{a}$ \\
\hline Total & 8.87 & 8.85 & 8.86 & 8.93 & 8.85 & 8.85 & 8.87 & 8.81 & 8.80 & 8.86 & 8.81 & 8.8 \\
\hline & 16.00 & 16.00 & 16.00 & 16.00 & 16.00 & 16.00 & 16.00 & 16.00 & 16.00 & 16.00 & 16.00 & 16. \\
\hline
\end{tabular}


Manganese Oxides from USW G-4 2954-2955, continued

\begin{tabular}{|c|c|c|c|c|c|c|c|c|c|c|c|c|}
\hline \multirow[b]{2}{*}{$\mathrm{SiO}_{2}$} & \multicolumn{12}{|c|}{ Cryptomelane-Hollandite $+/$ - Todorokite } \\
\hline & 0.30 & 0.39 & 0.24 & 0.84 & 0.62 & 0.33 & 0.28 & 0.44 & 0.31 & 0.37 & 0.34 & 0.46 \\
\hline $\mathrm{TiO}_{2}$ & 0.37 & 0.67 & 0.20 & 0.45 & 0.62 & 0.33 & 0.18 & 0.49 & 0.30 & 0.41 & 0.60 & 0.94 \\
\hline $\mathrm{MnO}_{2}$ & 73.34 & 81.03 & 78.89 & 70.26 & 71.11 & 78.79 & 79.55 & 74.36 & 75.71 & 77.58 & 78.24 & 69.44 \\
\hline $\mathrm{CeO}_{2}$ & $\mathrm{n} / \mathrm{a}$ & - & - & - & - & - & - & n/a & - & - & 0.21 & - \\
\hline $\mathrm{Al}_{2} \mathrm{O}_{3}$ & 0.41 & 0.62 & 0.18 & 0.52 & 0.35 & 0.39 & 0.22 & 0.49 & 0.35 & 0.41 & 0.52 & 0.48 \\
\hline $\mathrm{Fe}_{2} \mathrm{O}_{3}$ & 2.70 & 3.58 & 2.38 & 3.54 & 4.21 & 2.69 & 1.99 & 2.94 & 2.78 & 2.71 & 3.01 & 4.28 \\
\hline $\mathrm{MgO}$ & 0.59 & 0.20 & 1.29 & 0.22 & 0.45 & 0.82 & 1.30 & 0.76 & 0.90 & 0.92 & 0.50 & 0.07 \\
\hline $\mathrm{CaO}$ & 0.76 & 0.35 & 1.13 & 0.85 & 0.95 & 0.90 & 1.18 & 1.01 & 1.00 & 0.93 & 0.80 & 0.37 \\
\hline $\mathrm{ZnO}$ & - & - & 0.25 & 0.16 & 0.31 & 0.26 & - & 0.19 & - & - & - & - \\
\hline SrO & 1.11 & 1.03 & 1.08 & 1.33 & 1.67 & 1.20 & 1.23 & 0.91 & 1.31 & 1.20 & 1.02 & 1.19 \\
\hline $\mathrm{BaO}$ & 4.27 & 4.28 & 4.43 & 4.51 & 4.55 & 4.59 & 4.61 & 4.63 & 4.65 & 4.71 & 4.76 & 4.78 \\
\hline $\mathrm{PbO}$ & - & $\mathrm{n} / \mathrm{a}$ & $\mathrm{n} / \mathrm{a}$ & $\mathrm{n} / \mathrm{a}$ & $\mathrm{n} / \mathrm{a}$ & $\mathrm{n} / \mathrm{a}$ & $\mathrm{n} / \mathrm{a}$ & - & $\mathrm{n} / \mathrm{a}$ & $\mathrm{n} / \mathrm{a}$ & - & $\mathrm{n} / \mathrm{a}$ \\
\hline $\mathrm{Na}_{2} \mathrm{O}$ & 0.89 & 0.53 & 0.97 & 0.58 & 0.75 & 0.85 & 1.05 & 0.78 & 0.99 & 0.86 & 0.63 & 0.47 \\
\hline $\mathrm{K}_{2} \mathrm{O}$ & 2.35 & 3.75 & 1.28 & 2.41 & 1.67 & 2.09 & 1.37 & 2.05 & 1.75 & 1.71 & 2.64 & 2.90 \\
\hline $\mathrm{La}_{2} \mathrm{O}_{3}$ & - & n/a & n/a & n/a & n/a & n/a & $\mathrm{n} / \mathrm{a}$ & - & $\mathrm{n} / \mathrm{a}$ & $\mathrm{n} / \mathrm{a}$ & - & n/a \\
\hline Total & 87.09 & 96.43 & 92.32 & 85.67 & 87.26 & 93.24 & 92.96 & 89.05 & 90.05 & 91.81 & 93.27 & 85.38 \\
\hline $\mathrm{Si}^{+4}$ & 0.04 & 0.05 & 0.03 & 0.12 & 0.09 & 0.04 & 0.04 & 0.06 & 0.04 & 0.05 & 0.05 & 0.07 \\
\hline $\mathrm{Ti}^{+4}$ & 0.04 & 0.06 & 0.02 & 0.05 & 0.07 & 0.03 & 0.02 & 0.05 & 0.03 & 0.04 & 0.06 & 0.10 \\
\hline $\mathrm{Mn}^{+4}$ & 7.20 & 7.18 & 7.25 & 7.04 & 7.00 & 7.21 & 7.27 & 7.13 & 7.18 & 7.20 & 7.18 & 7.02 \\
\hline $\mathrm{Ce}^{+4}$ & $\mathrm{n} / \mathrm{a}$ & - & - & - & - & - & - & $\mathrm{n} / \mathrm{a}$ & - & - & 0.01 & - \\
\hline $\mathrm{Al}^{+3}$ & 0.07 & 0.09 & 0.03 & 0.09 & 0.06 & 0.06 & 0.03 & 0.08 & 0.06 & 0.06 & 0.08 & 0.08 \\
\hline $\mathrm{Fe}^{+3}$ & 0.29 & 0.35 & 0.24 & 0.39 & 0.45 & 0.27 & 0.20 & 0.31 & 0.29 & 0.27 & 0.30 & 0.47 \\
\hline $\mathrm{Mg}^{+2}$ & 0.12 & 0.04 & 0.26 & 0.05 & 0.10 & 0.16 & 0.26 & 0.16 & 0.18 & 0.18 & 0.10 & 0.02 \\
\hline $\mathrm{Ca}^{+2}$ & 0.12 & 0.05 & 0.16 & 0.13 & 0.15 & 0.13 & 0.17 & 0.15 & 0.15 & 0.13 & 0.11 & 0.06 \\
\hline $\mathrm{Zn}^{+2}$ & - & - & 0.02 & 0.02 & 0.03 & 0.03 & - & 0.02 & - & - & - & - \\
\hline $\mathrm{Sr}^{+2}$ & 0.09 & 0.08 & 0.08 & 0.11 & 0.14 & 0.09 & 0.09 & 0.07 & 0.10 & 0.09 & 0.08 & 0.10 \\
\hline $\mathrm{Ba}^{+2}$ & 0.24 & 0.22 & 0.23 & 0.26 & 0.25 & 0.24 & 0.24 & 0.25 & 0.25 & 0.25 & 0.25 & 0.27 \\
\hline $\mathrm{Pb}^{+2}$ & - & $\mathrm{n} / \mathrm{a}$ & $\mathrm{n} / \mathrm{a}$ & $\mathrm{n} / \mathrm{a}$ & $\mathrm{n} / \mathrm{a}$ & $\mathrm{n} / \mathrm{a}$ & $\mathrm{n} / \mathrm{a}$ & - & $\mathrm{n} / \mathrm{a}$ & n/a & - & $\mathrm{n} / \mathrm{a}$ \\
\hline $\mathrm{Na}^{+1}$ & 0.25 & 0.13 & 0.25 & 0.16 & 0.21 & 0.22 & 0.27 & 0.21 & 0.26 & 0.22 & 0.16 & 0.13 \\
\hline $\mathrm{K}^{+1}$ & 0.43 & 0.61 & 0.22 & 0.45 & 0.30 & 0.35 & 0.23 & 0.36 & 0.31 & 0.29 & 0.45 & 0.54 \\
\hline $\mathrm{La}^{+3}$ & - & $\mathrm{n} / \mathrm{a}$ & $\mathrm{n} / \mathrm{a}$ & $\mathrm{n} / \mathrm{a}$ & $\mathrm{n} / \mathrm{a}$ & $\mathrm{n} / \mathrm{a}$ & $\mathrm{n} / \mathrm{a}$ & - & $\mathrm{n} / \mathrm{a}$ & $\mathrm{n} / \mathrm{a}$ & - & $n / a$ \\
\hline Total & 8.88 & 8.86 & 8.80 & 8.86 & 8.84 & 8.83 & 8.81 & 8.85 & 8.86 & 8.80 & 8.82 & 8.87 \\
\hline & 16.00 & 16.00 & 16.00 & 16.00 & 16.00 & 16.00 & 16.00 & 16.00 & 16.00 & 16.00 & 16.00 & 16.00 \\
\hline
\end{tabular}


Manganese Oxides from USW G-4 2954-2955, continued

Cryptomelane-Hollandite $+/$ - Todorokite

\begin{tabular}{|c|c|c|c|c|c|c|c|c|c|c|c|c|}
\hline $\mathrm{SiO}_{2}$ & 0.53 & 0.25 & 0.47 & 0.82 & 0.53 & 0.47 & 0.23 & 0.81 & 0.23 & 0.35 & 0.33 & 0.77 \\
\hline $\mathrm{TiO}_{2}$ & 0.37 & - & 0.87 & 0.46 & 0.35 & 0.49 & 0.19 & 0.39 & 0.14 & 0.26 & 0.36 & 0.48 \\
\hline $\mathrm{MnO}_{2}$ & 75.39 & 77.16 & 70.13 & 69.27 & 72.53 & 72.06 & 75.89 & 72.92 & 79.80 & 74.65 & 75.45 & 73.82 \\
\hline $\mathrm{CeO}_{2}$ & - & - & - & - & - & - & - & - & - & $\mathrm{n} / \mathrm{a}$ & - & - \\
\hline $\mathrm{Al}_{2} \mathrm{O}_{3}$ & 0.33 & 0.17 & 0.47 & 0.33 & 0.21 & 0.33 & 0.26 & 0.34 & 0.18 & 0.18 & 0.34 & 0.40 \\
\hline $\mathrm{Fe}_{2} \mathrm{O}_{3}$ & 3.47 & 2.23 & 4.44 & 4.36 & 3.98 & 3.49 & 2.24 & 4.14 & 2.43 & 3.03 & 3.22 & 5.17 \\
\hline $\mathrm{MgO}$ & 1.02 & 1.63 & 0.08 & 0.81 & 1.14 & 1.14 & 1.52 & 0.72 & 1.18 & 1.50 & 1.58 & 0.88 \\
\hline $\mathrm{CaO}$ & 1.11 & 1.37 & 0.37 & 1.09 & 1.42 & 1.11 & 1.34 & 1.22 & 1.22 & 1.28 & 1.44 & 1.54 \\
\hline Zno & - & - & - & 0.25 & - & - & - & 0.26 & 0.34 & 0.15 & - & - \\
\hline Sro & 1.30 & 0.88 & 1.13 & 1.23 & 1.32 & 1.09 & 0.78 & 1.60 & 1.44 & 0.87 & 0.62 & 1.06 \\
\hline $\mathrm{BaO}$ & 4.79 & 4.81 & 4.82 & 4.87 & 4.98 & 5.08 & 5.08 & 5.19 & 5.23 & 5.35 & 6.10 & 6.21 \\
\hline $\mathrm{PbO}$ & $\mathrm{n} / \mathrm{a}$ & $\mathrm{n} / \mathrm{a}$ & $n / a$ & $\mathrm{n} / \mathrm{a}$ & $\mathrm{n} / \mathrm{a}$ & n/a & 0.11 & $\mathrm{n} / \mathrm{a}$ & $\mathrm{n} / \mathrm{a}$ & - & - & 0.14 \\
\hline $\mathrm{Na}_{2} \mathrm{O}$ & 0.90 & 1.06 & 0.48 & 0.70 & 0.91 & 0.78 & 0.97 & 0.69 & 0.86 & 1.13 & 0.94 & 0.84 \\
\hline $\mathrm{K}_{2} \mathrm{O}$ & 1.19 & 0.84 & 3.05 & 1.24 & 0.73 & 1.03 & 0.70 & 1.32 & 1.06 & 0.62 & 0.41 & 0.81 \\
\hline $\mathrm{La}_{2} \mathrm{O}_{3}$ & $\mathrm{n} / \mathrm{a}$ & $\mathrm{n} / \mathrm{a}$ & $\mathrm{n} / \mathrm{a}$ & $\mathrm{n} / \mathrm{a}$ & $\mathrm{n} / \mathrm{a}$ & $\mathrm{n} / \mathrm{a}$ & - & n/a & $\mathrm{n} / \mathrm{a}$ & - & - & 0.11 \\
\hline Total & 90.40 & 90.40 & 86.31 & 85.43 & 88.10 & 87.07 & 89.31 & 89.60 & 94.11 & 89.37 & 90.79 & 92.23 \\
\hline $\mathrm{Si}^{+4}$ & 0.07 & 0.03 & 0.07 & 0.12 & 0.07 & 0.07 & 0.03 & 0.11 & 0.03 & 0.05 & 0.04 & 0.10 \\
\hline $\mathrm{Ti}^{+4}$ & 0.04 & - & 0.09 & 0.05 & 0.04 & 0.05 & 0.02 & 0.04 & 0.01 & 0.03 & 0.04 & 0.05 \\
\hline $\mathrm{Mn}^{+4}$ & 7.11 & 7.24 & 7.02 & 6.95 & 7.04 & 7.07 & 7.22 & 6.99 & 7.25 & 7.13 & 7.10 & 6.90 \\
\hline $\mathrm{Ce}^{+4}$ & - & - & - & - & - & - & - & - & - & $\mathrm{n} / \mathrm{a}$ & - & - \\
\hline $\mathrm{Al}^{+3}$ & 0.05 & 0.03 & 0.08 & 0.06 & 0.03 & 0.06 & 0.04 & 0.06 & 0.03 & 0.03 & 0.05 & 0.06 \\
\hline $\mathrm{Fe}^{+3}$ & 0.36 & 0.23 & 0.48 & 0.48 & 0.42 & 0.37 & 0.23 & 0.43 & 0.24 & 0.31 & 0.33 & 0.53 \\
\hline $\mathrm{Mg}^{+2}$ & 0.21 & 0.33 & 0.02 & 0.18 & 0.24 & 0.24 & 0.31 & 0.15 & 0.23 & 0.31 & 0.32 & 0.18 \\
\hline $\mathrm{Ca}^{+2}$ & 0.16 & 0.20 & 0.06 & 0.17 & 0.21 & 0.17 & 0.20 & 0.18 & 0.17 & 0.19 & 0.21 & 0.22 \\
\hline $\mathrm{Zn}^{+2}$ & - & - & - & 0.03 & - & - & - & 0.03 & 0.03 & 0.02 & - & - \\
\hline $\mathrm{Sr}^{+2}$ & 0.10 & 0.07 & 0.09 & 0.10 & 0.11 & 0.09 & 0.06 & 0.13 & 0.11 & 0.07 & 0.05 & 0.08 \\
\hline $\mathrm{Ba}^{+2}$ & 0.26 & 0.26 & 0.27 & 0.28 & 0.27 & 0.28 & 0.27 & 0.28 & 0.27 & 0.29 & 0.33 & 0.33 \\
\hline $\mathrm{Pb}^{+2}$ & $\mathrm{n} / \mathrm{a}$ & $\mathrm{n} / \mathrm{a}$ & $\mathrm{n} / \mathrm{a}$ & $\mathrm{n} / \mathrm{a}$ & $n / a$ & $\mathrm{n} / \mathrm{a}$ & 0.00 & $\mathrm{n} / \mathrm{a}$ & $n / a$ & - & - & 0.01 \\
\hline $\mathrm{Na}^{+1}$ & 0.24 & 0.28 & 0.13 & 0.20 & 0.25 & 0.21 & 0.26 & 0.19 & 0.22 & 0.30 & 0.25 & 0.22 \\
\hline $\mathrm{K}^{+1}$ & 0.21 & 0.15 & 0.56 & 0.23 & 0.13 & 0.19 & 0.12 & 0.23 & 0.18 & 0.11 & 0.07 & 0.14 \\
\hline $\mathrm{La}^{+3}$ & $\mathrm{n} / \mathrm{a}$ & $\mathrm{n} / \mathrm{a}$ & $\mathrm{n} / \mathrm{a}$ & $\mathrm{n} / \mathrm{a}$ & $\mathrm{n} / \mathrm{a}$ & $\mathrm{n} / \mathrm{a}$ & - & $\mathrm{n} / \mathrm{a}$ & $\mathrm{n} / \mathrm{a}$ & - & - & 0.01 \\
\hline Total & 8.80 & 8.81 & 8.89 & 8.83 & 8.81 & 8.80 & 8.78 & 8.82 & 8.77 & 8.83 & 8.79 & 8.83 \\
\hline $\mathrm{O}^{-2}$ & 16.00 & 16.00 & 16.00 & 16.00 & 16.00 & 16.00 & 16.00 & 16.00 & 16.00 & 16.00 & 16.00 & 16.00 \\
\hline
\end{tabular}

${ }^{a}$ Analyzed for but not detected: $\mathrm{P}, \mathrm{Cl}, \mathrm{Co}, \mathrm{Cu}, \mathrm{Y}$, and $\mathrm{Ni}$ 
Cryptomelane

\begin{tabular}{|c|c|c|c|c|c|c|c|c|}
\hline $\mathrm{SiO}_{2}$ & 0.52 & 0.70 & 1.16 & 0.44 & 0.30 & 0.18 & 0.43 & 0.48 \\
\hline $\mathrm{TiO}_{2}$ & 1.15 & 1.10 & 1.18 & 0.41 & 0.32 & 0.21 & 0.57 & 0.50 \\
\hline $\mathrm{MnO}_{2}$ & 73.49 & 75.29 & 69.02 & 78.63 & 79.97 & 80.70 & 74.41 & 76.76 \\
\hline $\mathrm{CeO}_{2}$ & - & $\mathrm{n} / \mathrm{a}$ & $\mathrm{n} / \mathrm{a}$ & 1.81 & $\mathrm{n} / \mathrm{a}$ & - & 0.53 & n/a \\
\hline $\mathrm{Al}_{2} \mathrm{O}_{3}$ & 0.67 & 0.64 & 0.81 & 0.38 & 0.38 & 0.49 & 0.46 & 0.43 \\
\hline $\mathrm{Fe}_{2} \mathrm{O}_{3}$ & 4.01 & 4.15 & 4.44 & 2.75 & 1.87 & 1.78 & 2.68 & 2.72 \\
\hline $\mathrm{MgO}$ & 0.07 & 0.10 & 0.06 & 0.38 & 0.47 & 0.30 & 0.24 & 0.25 \\
\hline $\mathrm{CaO}$ & 0.47 & 0.42 & 0.45 & 0.89 & 0.79 & 0.73 & 0.73 & 0.71 \\
\hline $\mathrm{ZnO}$ & - & 0.16 & - & 0.22 & - & 0.23 & 0.21 & 0.16 \\
\hline SrO & 0.91 & 0.99 & 0.93 & 1.03 & 1.19 & 1.14 & 1.13 & 1.17 \\
\hline $\mathrm{BaO}$ & 5.54 & 5.86 & 6.07 & 6.12 & 6.30 & 6.40 & 7.18 & 7.45 \\
\hline $\mathrm{PbO}$ & - & - & - & 0.35 & - & 0.21 & 0.26 & 0.25 \\
\hline $\mathrm{Na}_{2} \mathrm{O}$ & 0.38 & 0.44 & 0.31 & 0.68 & 0.65 & 0.56 & 0.53 & 0.54 \\
\hline $\mathrm{K}_{2} \mathrm{O}$ & 2.69 & 3.05 & 2.64 & 2.15 & 2.24 & 2.43 & 1.61 & 2.11 \\
\hline $\mathrm{La}_{2} \mathrm{O}_{3}$ & - & - & - & - & - & - & - & 0.13 \\
\hline Total & 89.90 & 92.90 & 87.07 & 96.24 & 94.48 & 95.36 & 90.97 & 93.66 \\
\hline $\mathrm{Si}^{+4}$ & 0.07 & 0.09 & 0.17 & 0.06 & 0.04 & 0.02 & 0.06 & 0.06 \\
\hline $\mathrm{Ti}^{+4}$ & 0.12 & 0.11 & 0.13 & 0.04 & 0.03 & 0.02 & 0.06 & 0.05 \\
\hline $\mathrm{Mn}^{+4}$ & 7.04 & 7.00 & 6.85 & 7.13 & 7.30 & 7.33 & 7.15 & 7.16 \\
\hline $\mathrm{Ce}^{+4}$ & - & $\mathrm{n} / \mathrm{a}$ & $\mathrm{n} / \mathrm{a}$ & 0.08 & $\mathrm{n} / \mathrm{a}$ & - & 0.03 & $\mathrm{n} / \mathrm{a}$ \\
\hline $\mathrm{Al}^{+3}$ & 0.11 & 0.10 & 0.14 & 0.06 & 0.06 & 0.08 & 0.08 & 0.07 \\
\hline $\mathrm{Fe}^{+3}$ & 0.42 & 0.42 & 0.48 & 0.27 & 0.19 & 0.18 & 0.28 & 0.28 \\
\hline $\mathrm{Mg}^{+2}$ & 0.01 & 0.02 & 0.01 & 0.07 & 0.09 & 0.06 & 0.05 & 0.05 \\
\hline $\mathrm{Ca}^{+2}$ & 0.07 & 0.06 & 0.07 & 0.13 & 0.11 & 0.10 & 0.11 & 0.10 \\
\hline $\mathrm{Zn}^{+2}$ & - & 0.02 & - & 0.02 & - & 0.02 & 0.02 & 0.02 \\
\hline $\mathrm{Sr}^{+2}$ & 0.07 & 0.08 & 0.08 & 0.08 & 0.09 & 0.09 & 0.09 & 0.09 \\
\hline $\mathrm{Ba}^{+2}$ & 0.30 & 0.31 & 0.34 & 0.31 & 0.33 & 0.33 & 0.39 & 0.39 \\
\hline $\mathrm{Pb}^{+2}$ & - & - & - & 0.01 & - & 0.01 & 0.01 & 0.01 \\
\hline $\mathrm{Na}^{+1}$ & 0.10 & 0.11 & 0.09 & 0.17 & 0.17 & 0.14 & 0.14 & 0.14 \\
\hline $\mathrm{K}^{+1}$ & 0.48 & 0.52 & 0.48 & 0.36 & 0.38 & 0.41 & 0.29 & 0.36 \\
\hline $\mathrm{La}^{+3}$ & - & - & - & - & - & - & - & 0.01 \\
\hline Total & 8.79 & 8.85 & 8.83 & 8.79 & 8.78 & 8.78 & 8.75 & 8.80 \\
\hline $\mathrm{O}^{-2}$ & 16.00 & 16.00 & 16.00 & 16.00 & 16.00 & 16.00 & 16.00 & 16.00 \\
\hline
\end{tabular}

analyzed for but not detected: $\mathrm{Cl}, \mathrm{Y}$, and $\mathrm{Ni}$ 